




\section{LES LILIACEES;}

$1+\mathrm{An}$

P. J. REDOU TE. 



\title{
LES LILIACÉES;
}

PA R

P. J. R EDOUTÉ.

\author{
TOME PREMIER.
}

A PARIS,

CHEZ L'AUTEUR, RUE DE'L'ORATOIRE, HÓTEL D'ANGIVILLIERS.

DE L'IMPRIMERIE DE DIDOT JEUNE.

A N X III. - M D C C G V.

Mo. Bot. Garden, 


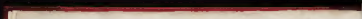




\section{PREFACE.}

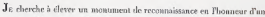

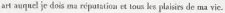

Luprunare des teurs, dide pour vinsi dire sons le siecle de Loais XIV,

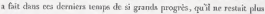

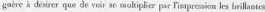

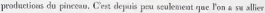

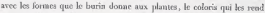

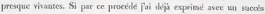

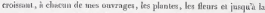

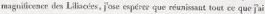

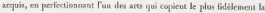

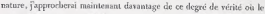
sols çui jage peut ère súthis

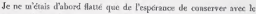

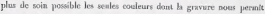
abors do buixe usage, Insiruit par l'esperitence, je publse tone seconde

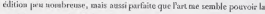

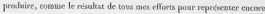

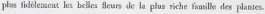

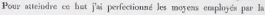

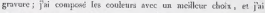

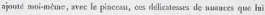

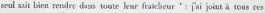

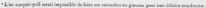




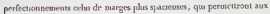
tiges de se deployer anee plis de graes el ile hiberté

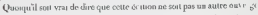

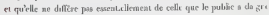

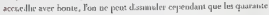

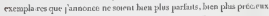

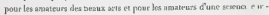

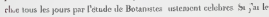
boahour fobieris les siurrages des wis ot des murea, vais urraex, qu.

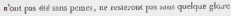




\title{
DIANELLA ENSIFOLIA.
}

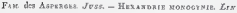

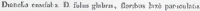

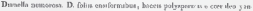

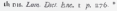

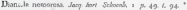

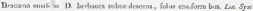

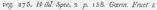

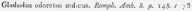

\section{DIANELEE EN GIAIVE}

\section{I S C I I T TON}

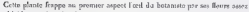

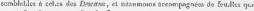

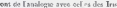

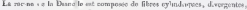

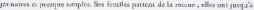

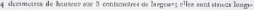

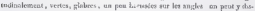

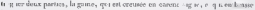

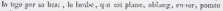

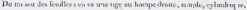

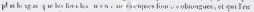

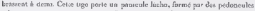

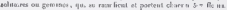

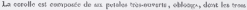

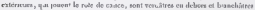

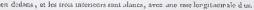
rex netion

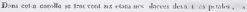

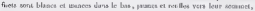

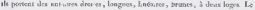

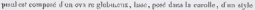

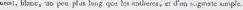




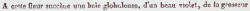

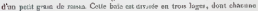

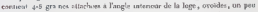

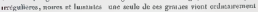

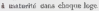

\section{H ISTOTRT}

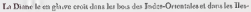

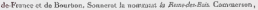

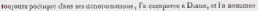

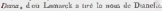

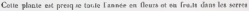

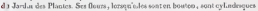

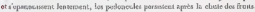

$$
\text { OABIRYATONS }
$$

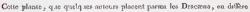

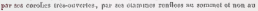

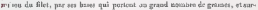
teat part ised port.

\section{EX PLICATIONDELA PIANCHI}

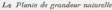

1 Los paribes de in fruciricalina

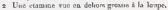

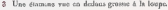

+ Lo pisilik

5 La bair.

6. Une dis grance. 



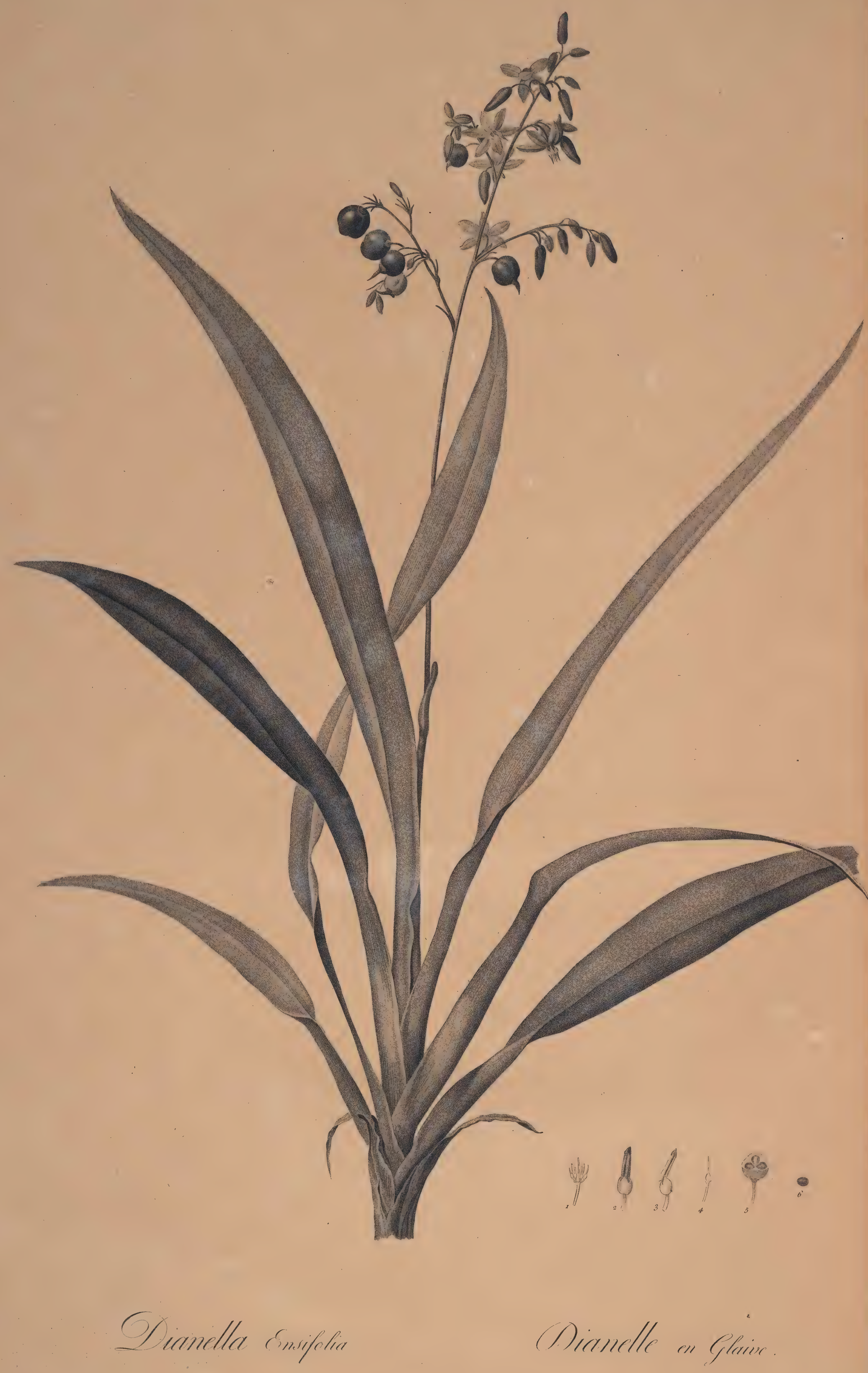




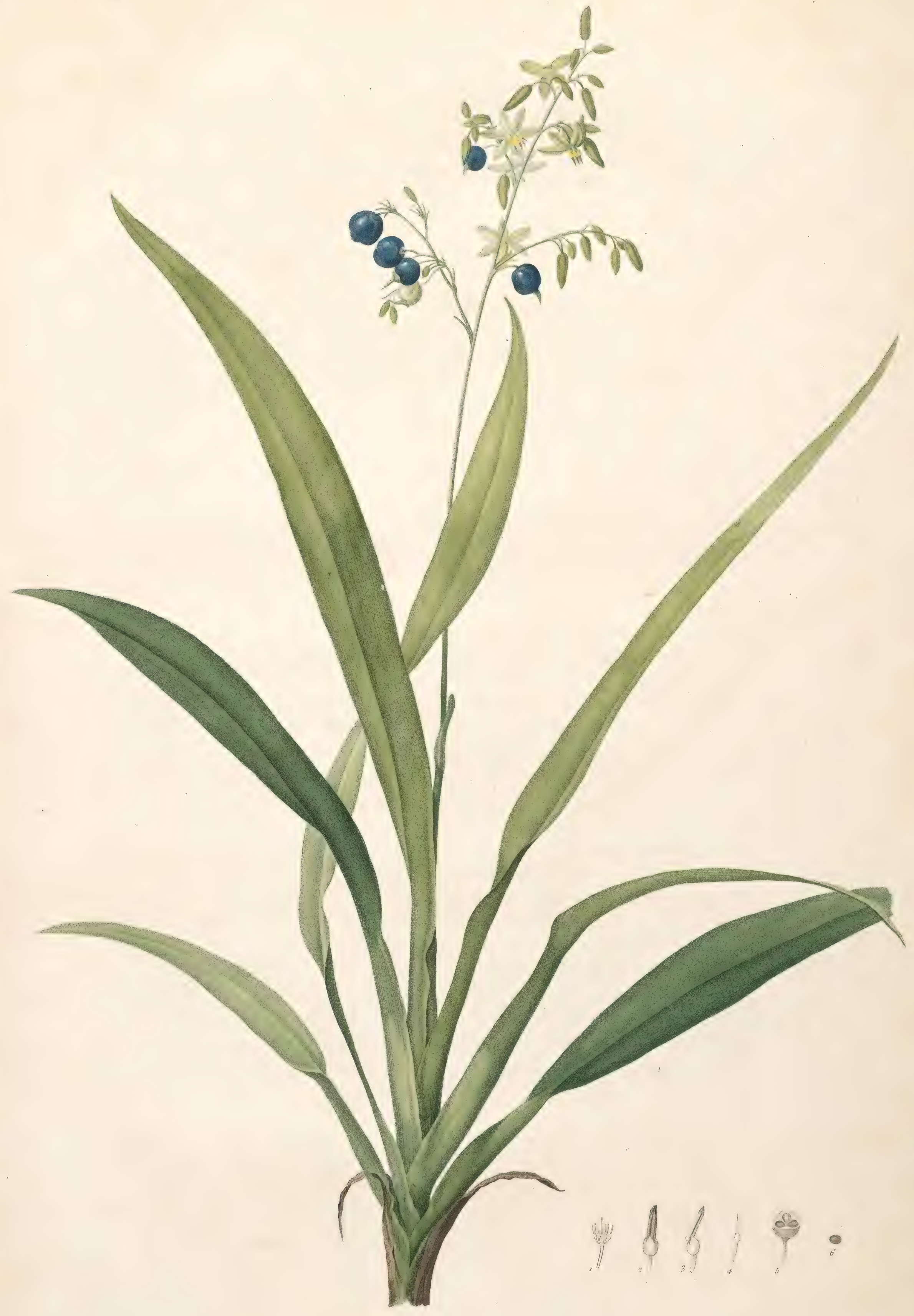

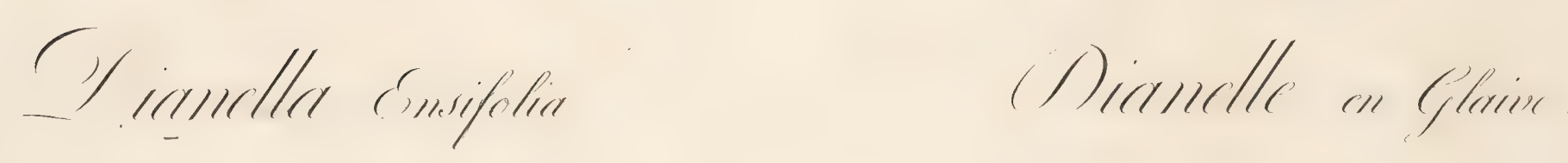




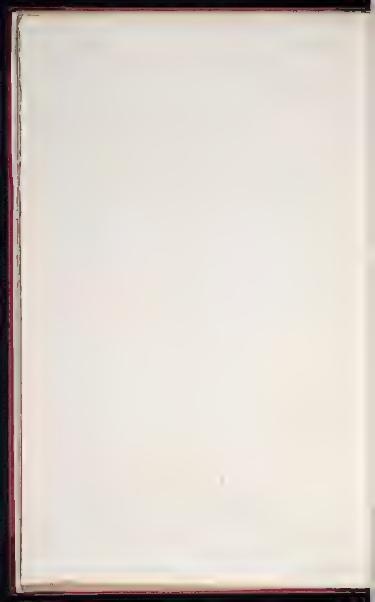




\section{LACIIENALIA TRICOLOR.}

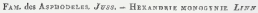

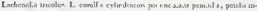

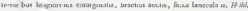
Bper, 175

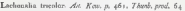

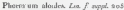

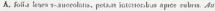

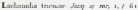

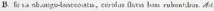

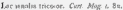

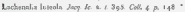

\section{ACHENALE THICOLOHE}

\section{DRSOกTETON}

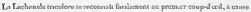

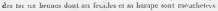

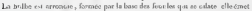

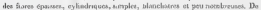

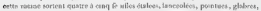

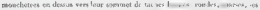

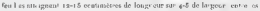

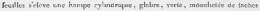

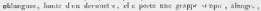

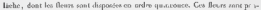

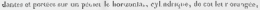

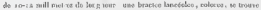

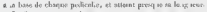

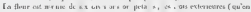

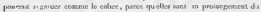

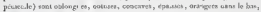

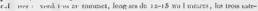

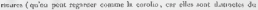

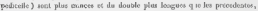




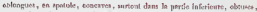

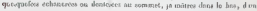
rage poupira av moterace

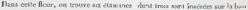

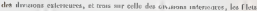

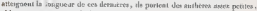

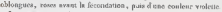

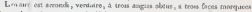

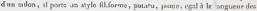

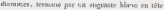

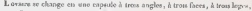

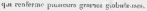

\section{UI STDIR I T}

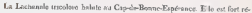

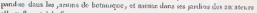

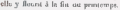

On to treot dies lo wre elinidr

\section{O B E K I A T O N}

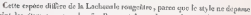

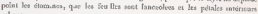
otlowe cons

\section{EXPLIGATION DI IA P I A C I I}

La Plave of groablour paliurtic

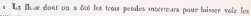
trim ace

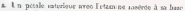

o. Le poutia. 



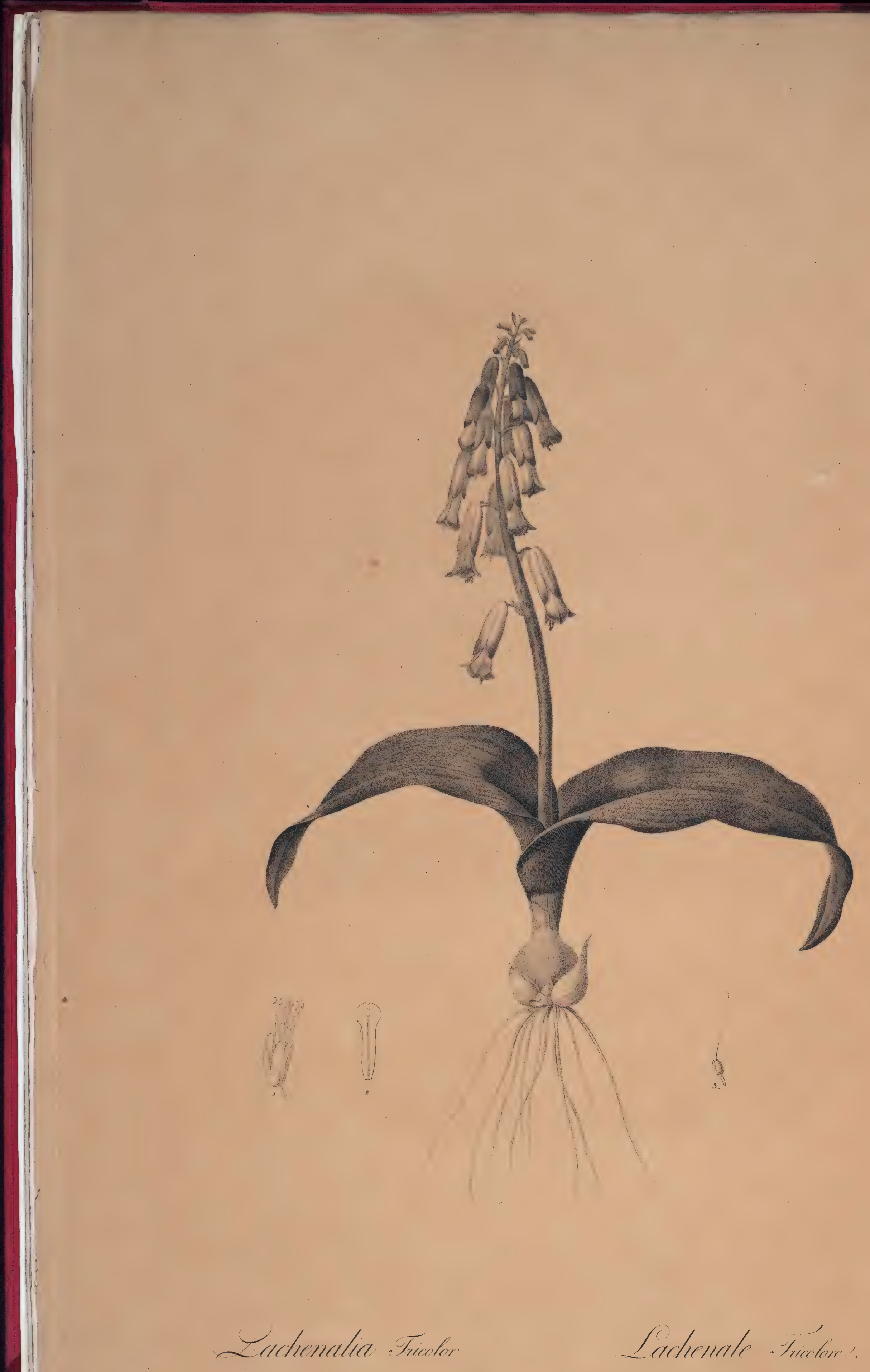




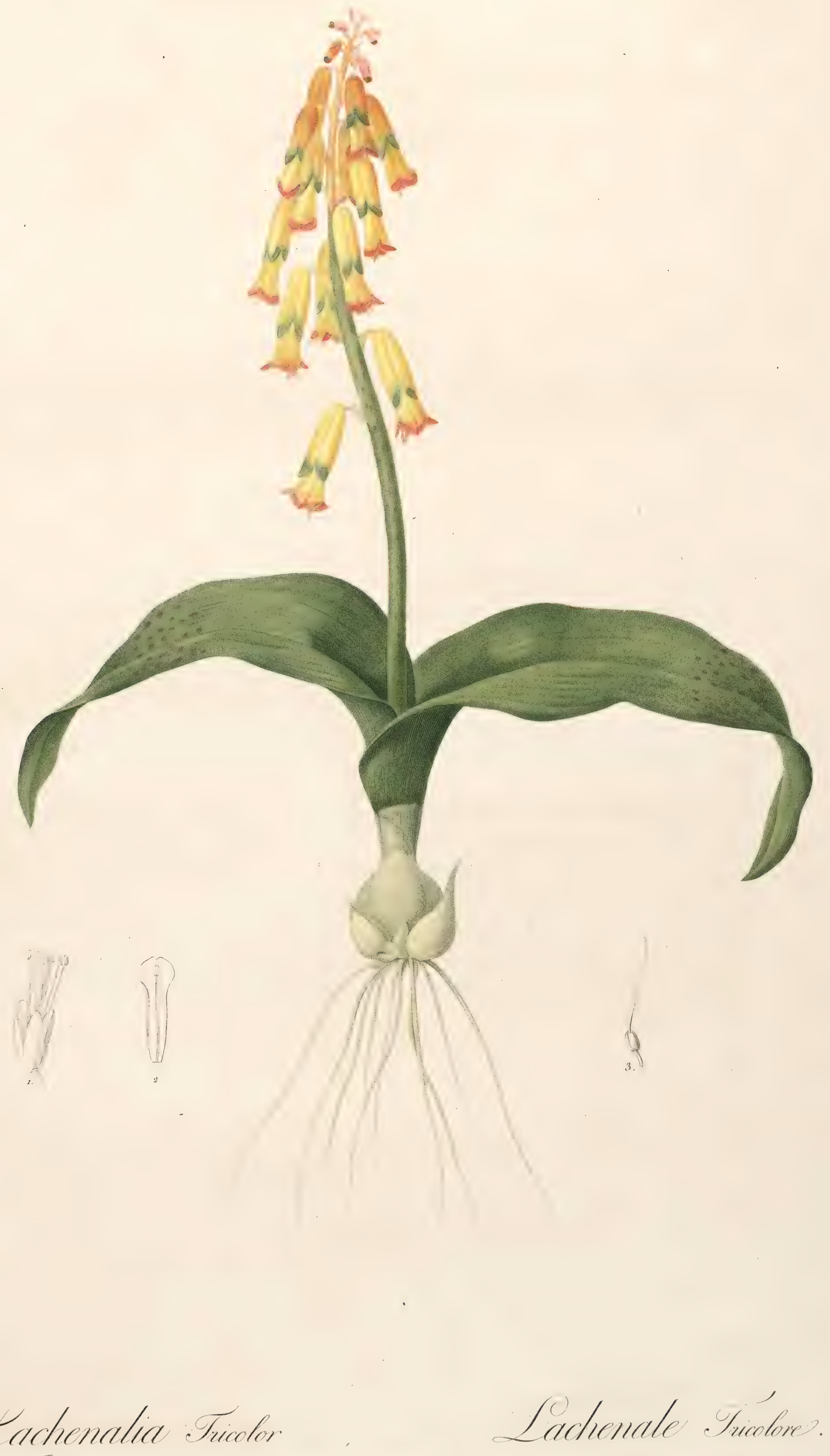

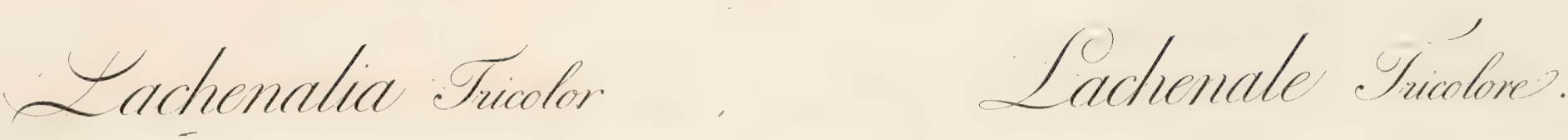




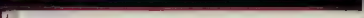




\title{
HEMEROCALLIS JAPONICA.
}

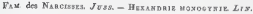

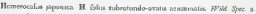
A. $1 g^{6}$

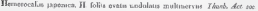

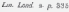

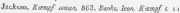

\section{HEVEMOCALLE DU JAPOY}

\section{ESC B I P T O N}

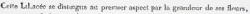

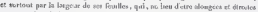

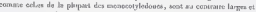
retreivis en pelects

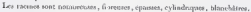

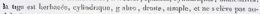

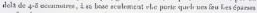

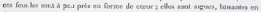

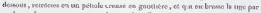

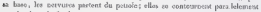

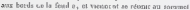

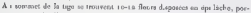

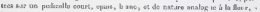

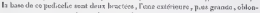

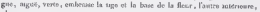

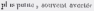

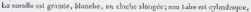

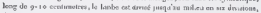

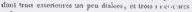

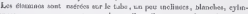

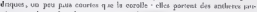

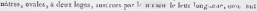

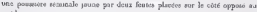
prat 


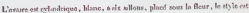

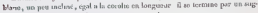

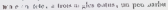

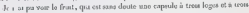
nitume

\section{T T I I E.}

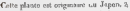

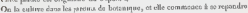

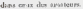

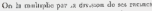

bile doit ibe pasks dane la worke ehieats

TXELICATION WR LA RLANCTL.

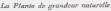

1. Corolte oarcite at diand 



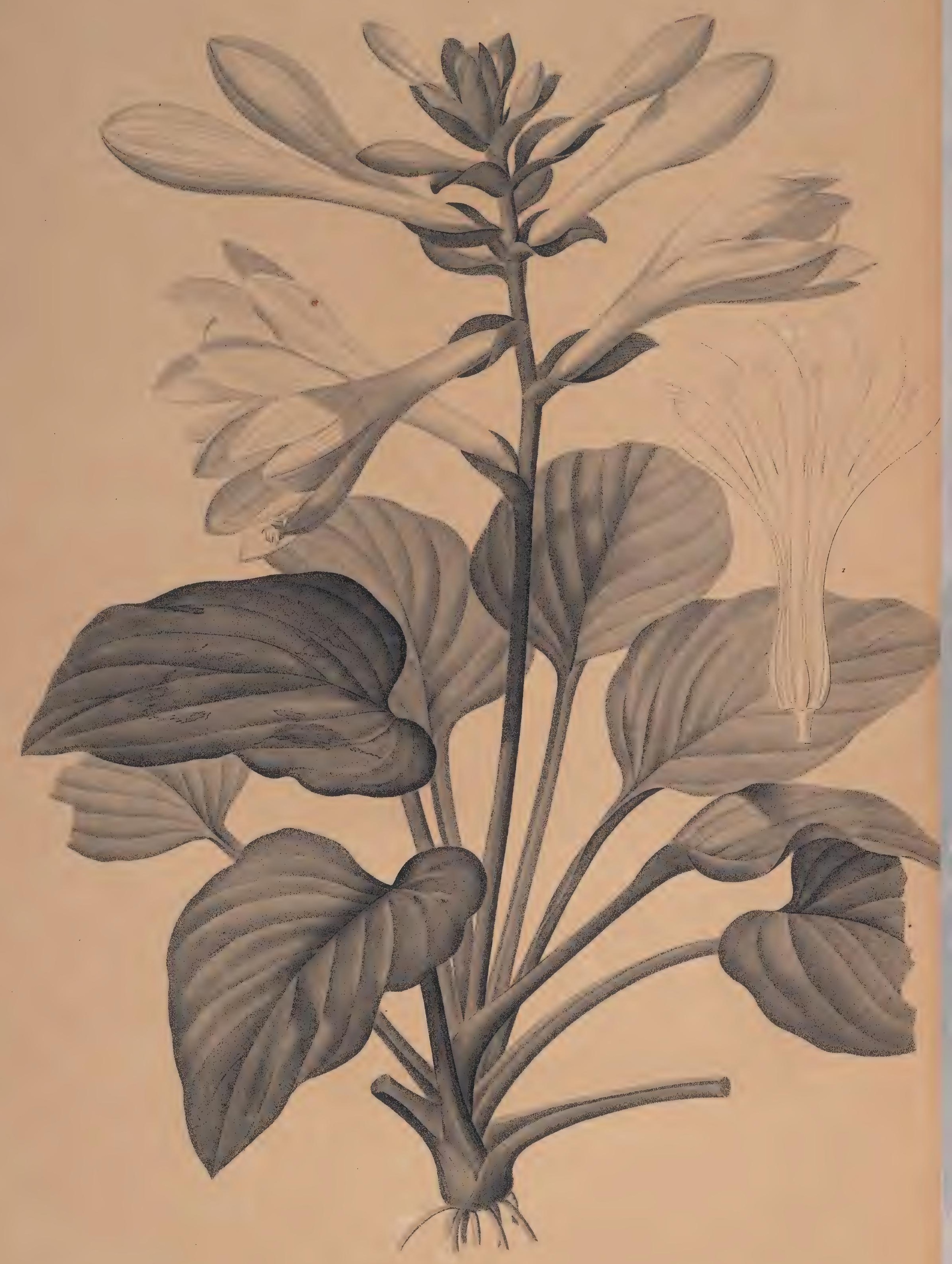

- Hemerocallis écupionica

Hemerocalle diu Gupipon 


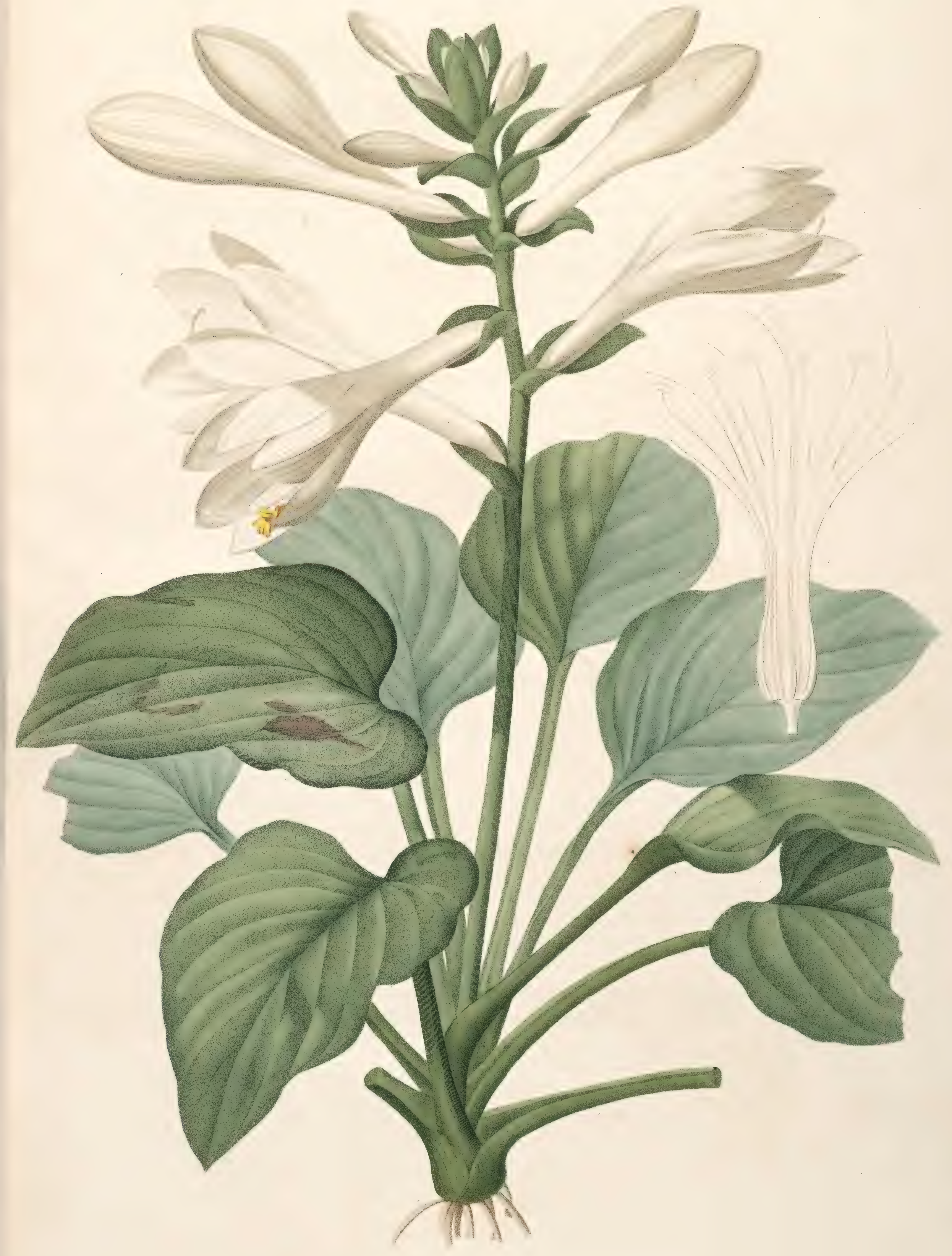

- Semerocentlis elefiponica

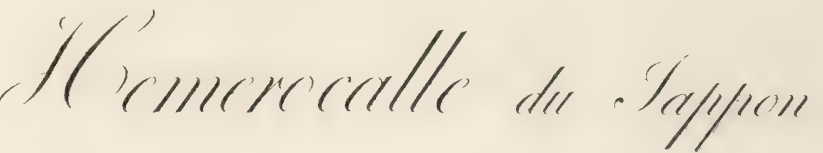




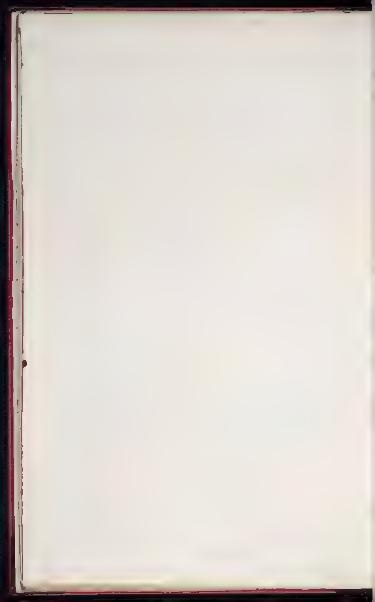




\title{
IG APANTIUS UMBELLATLS.
}

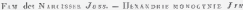

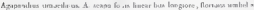

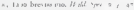

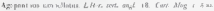

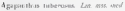

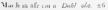

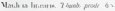

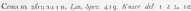

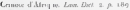

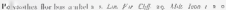

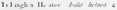

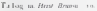

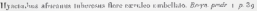

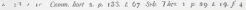

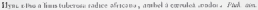
B? $1.05,8$,

\section{IG IPAVTIIE EV OUBFI LE}

\section{DTSERTETOX}

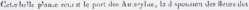

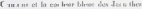

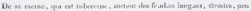

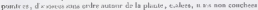
$n+1, m i$

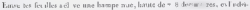

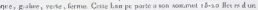

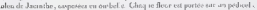

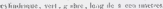

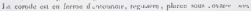

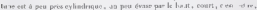

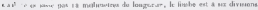

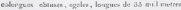

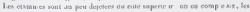

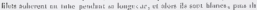




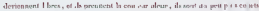

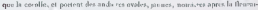

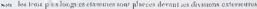

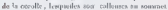

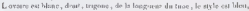

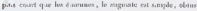

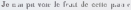

\section{$115 \$ 70191$}

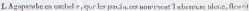

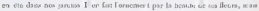

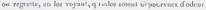

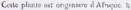




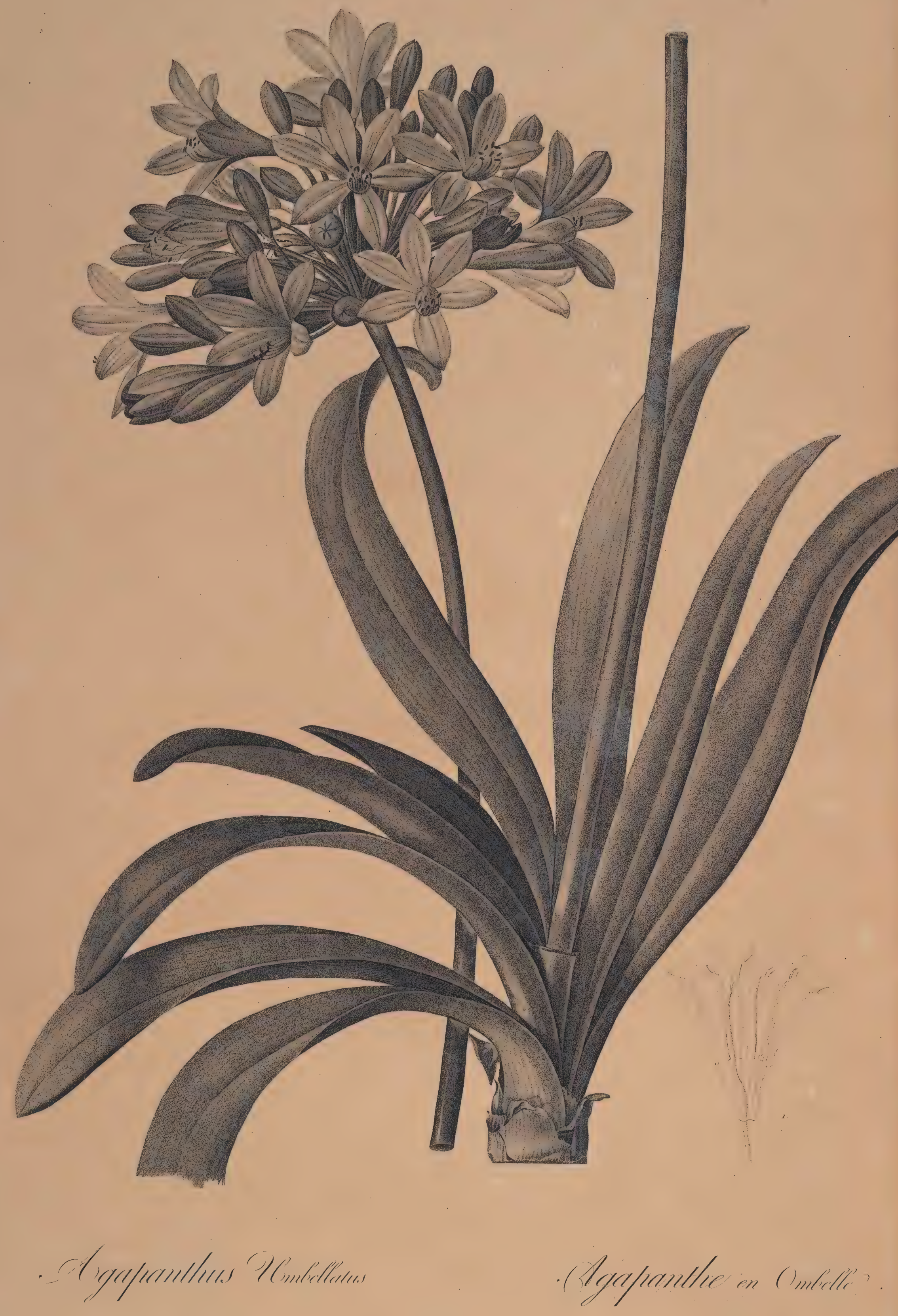




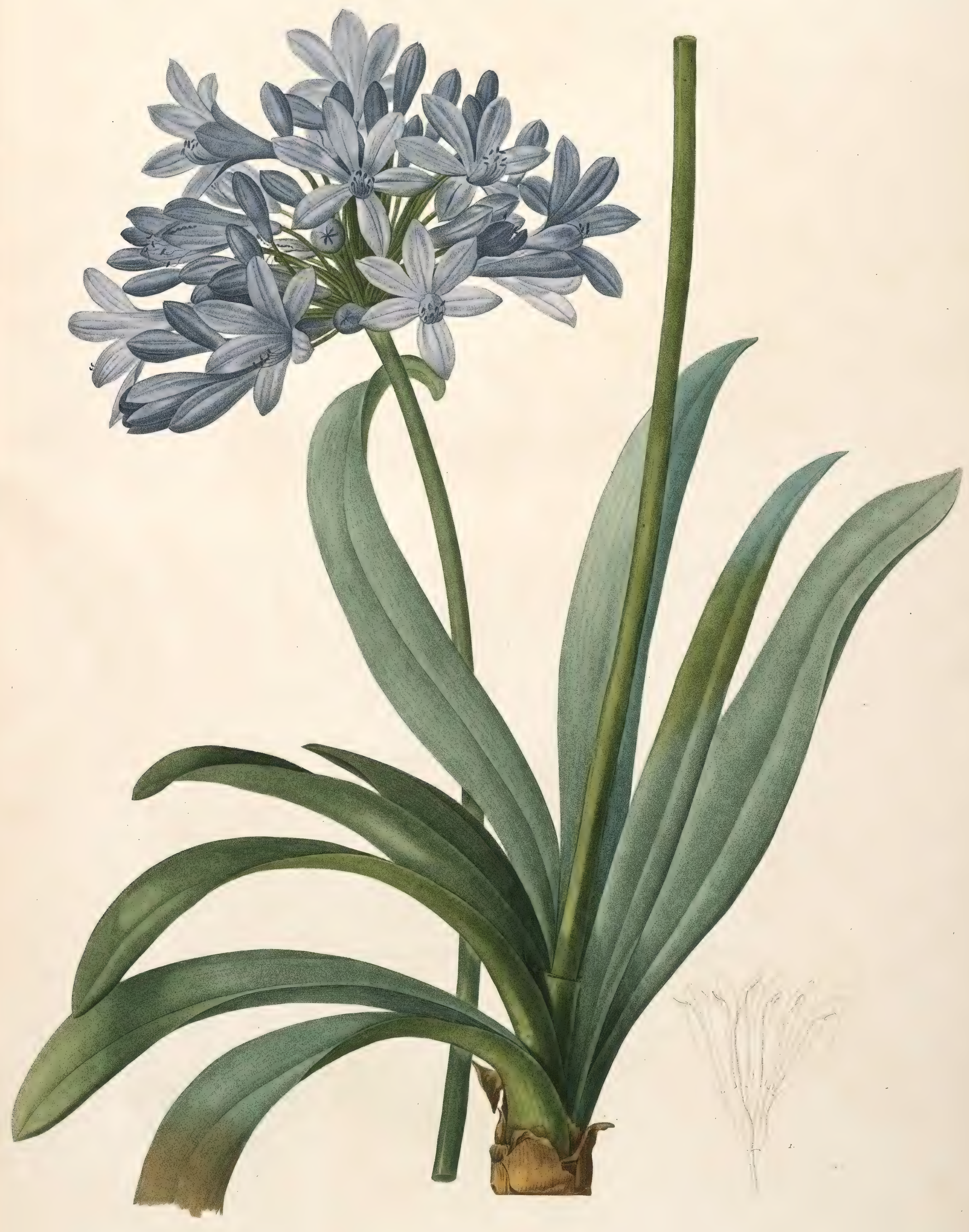

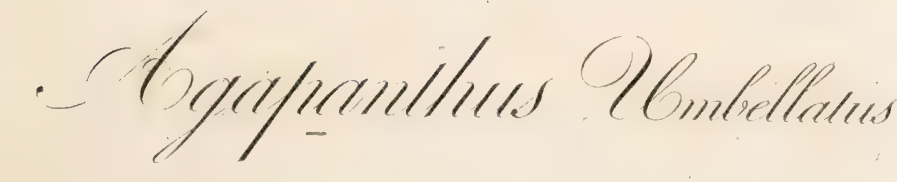

- Stgrefiencillie en Ombelles 


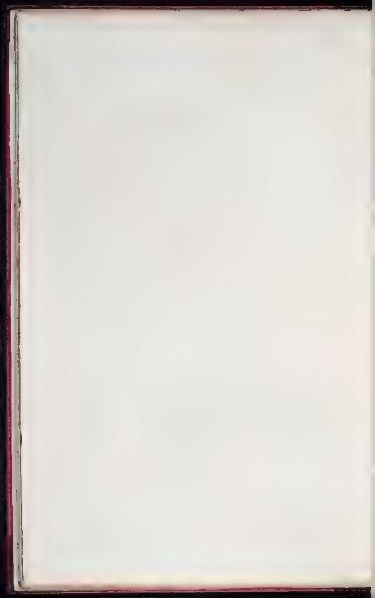




\title{
I YARYLLIS FORMOSISSIMA.
}

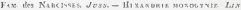

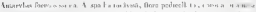

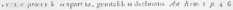

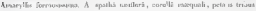

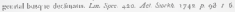

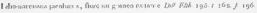

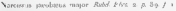

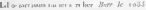

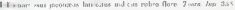

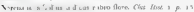

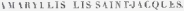

\section{DFFEIPTION}

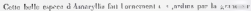

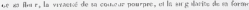

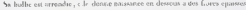

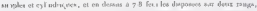

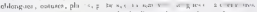

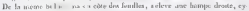

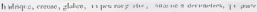

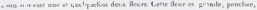

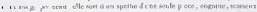

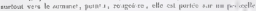

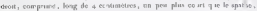

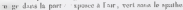

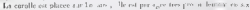

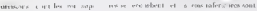

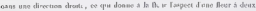

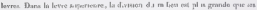

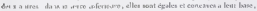

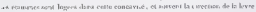

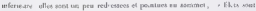




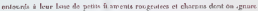
1 nime

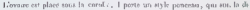

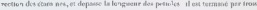

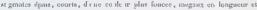
averimede

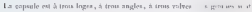

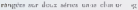

II $T$ T O IIT

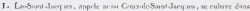

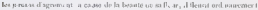

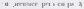

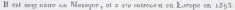





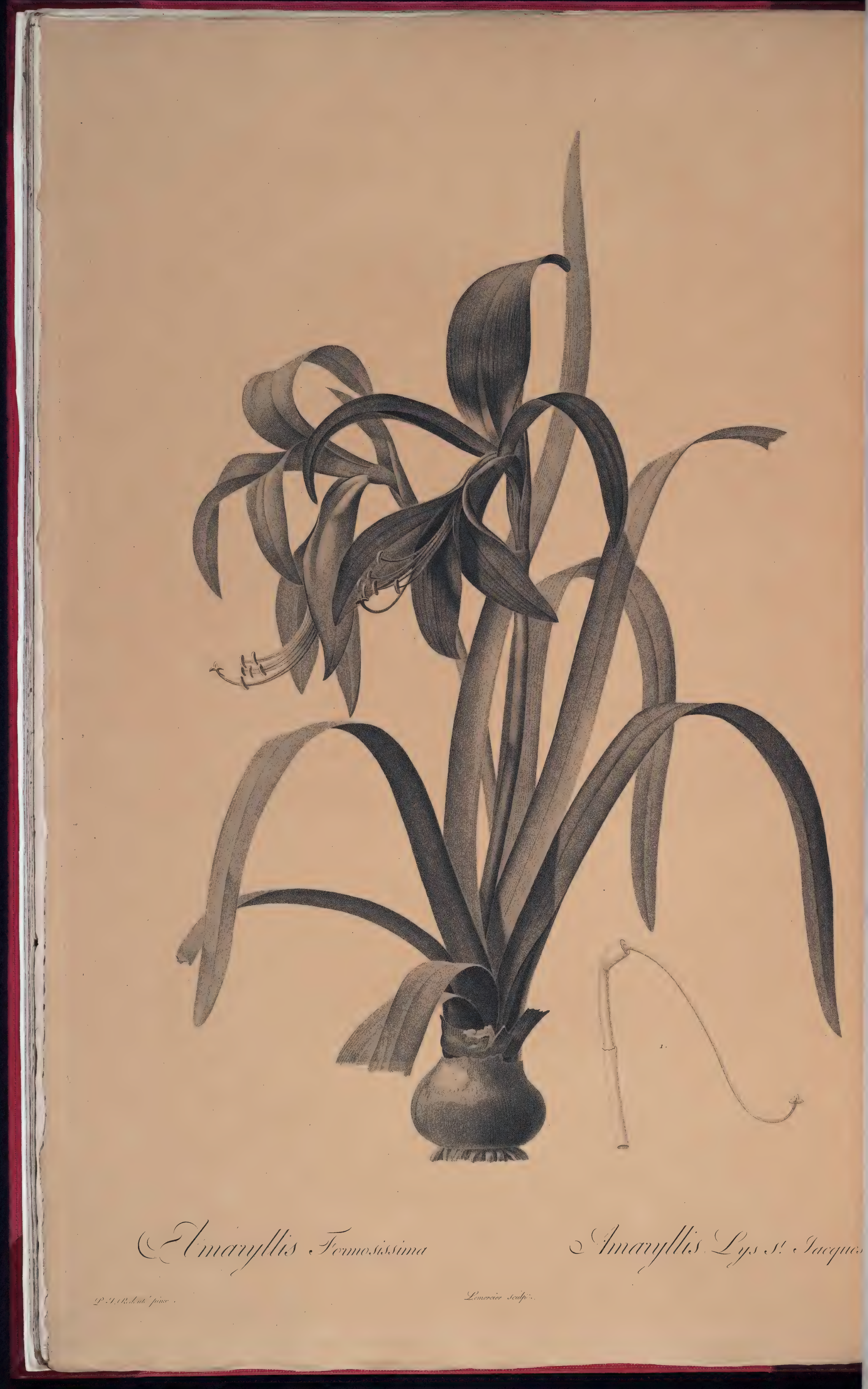




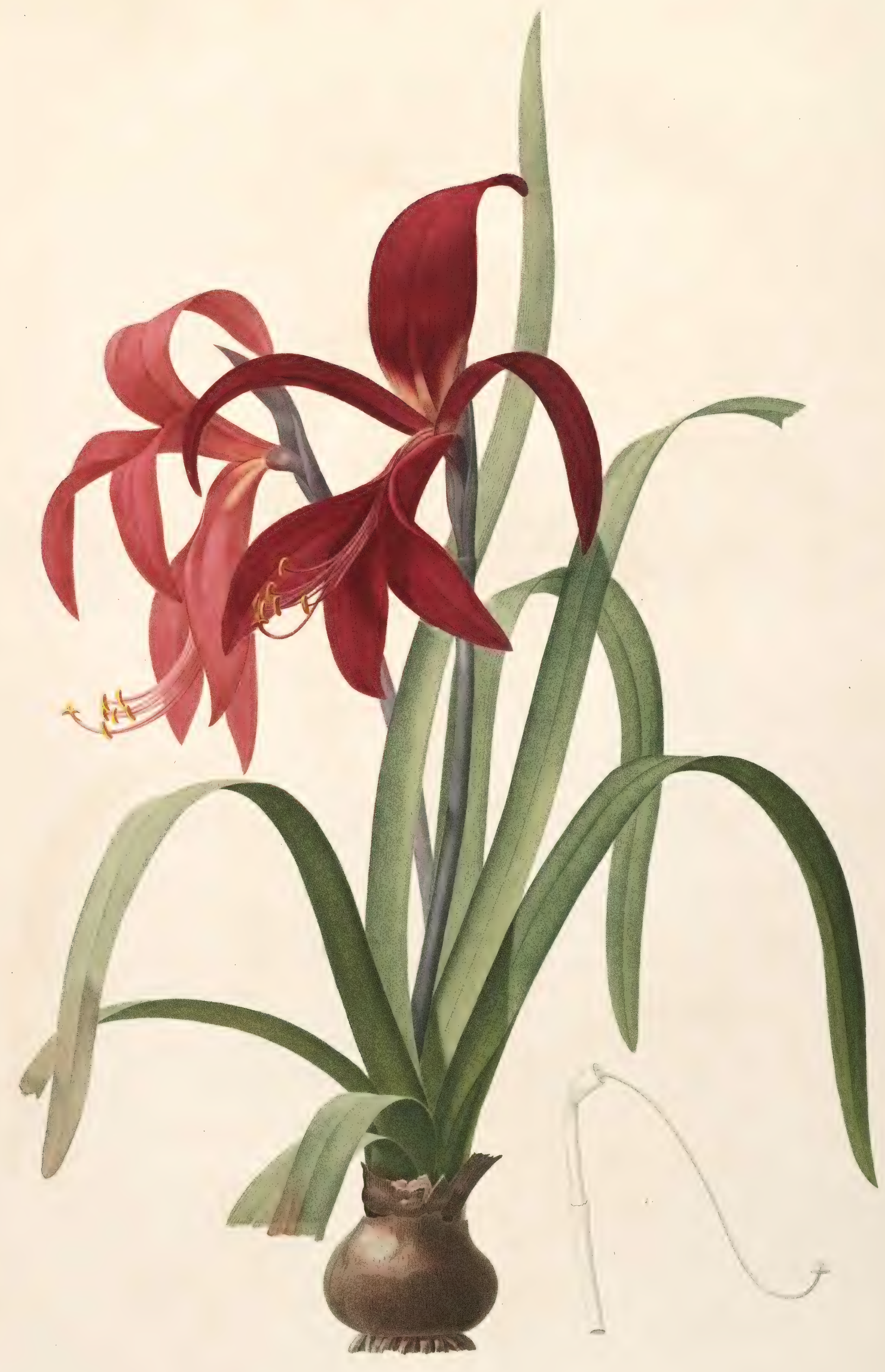

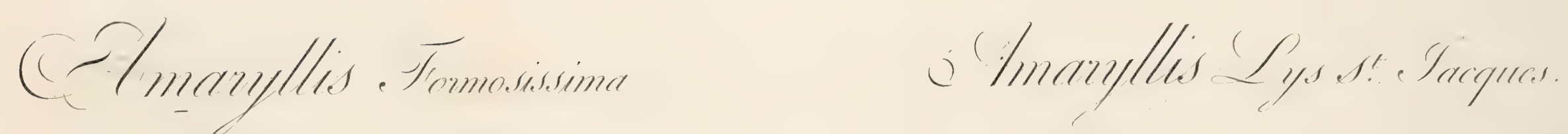




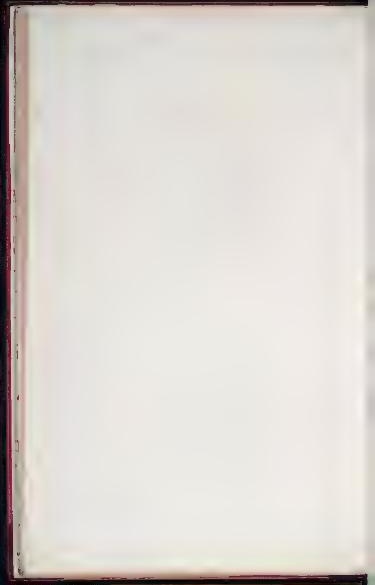




\title{
TIGRIDIA PAYONIA.
}

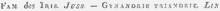

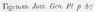

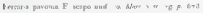

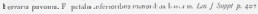

\section{TIGRIDIE QLECE DE PAON}

\section{ก $6.8 \mathrm{C}: \mathrm{H}+10 \mathrm{~K}$}

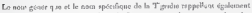

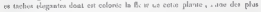

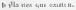

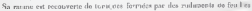

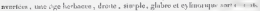

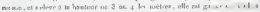

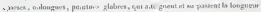

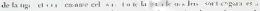

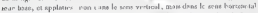

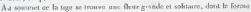

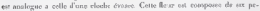

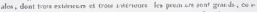

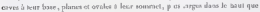

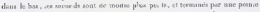

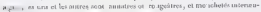

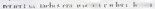

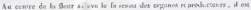

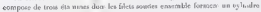

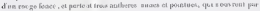

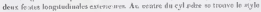

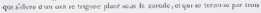

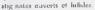

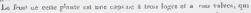

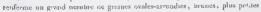

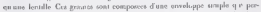

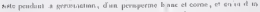

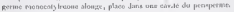




\section{I T T I R}

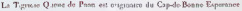

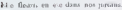

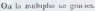

$$
O D S B A F d T R O N S
$$

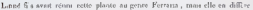

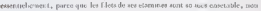

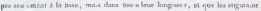
enint buluce, el twe en copegion.

\section{Y LICATION DE LA PLA M T T}

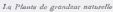

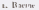




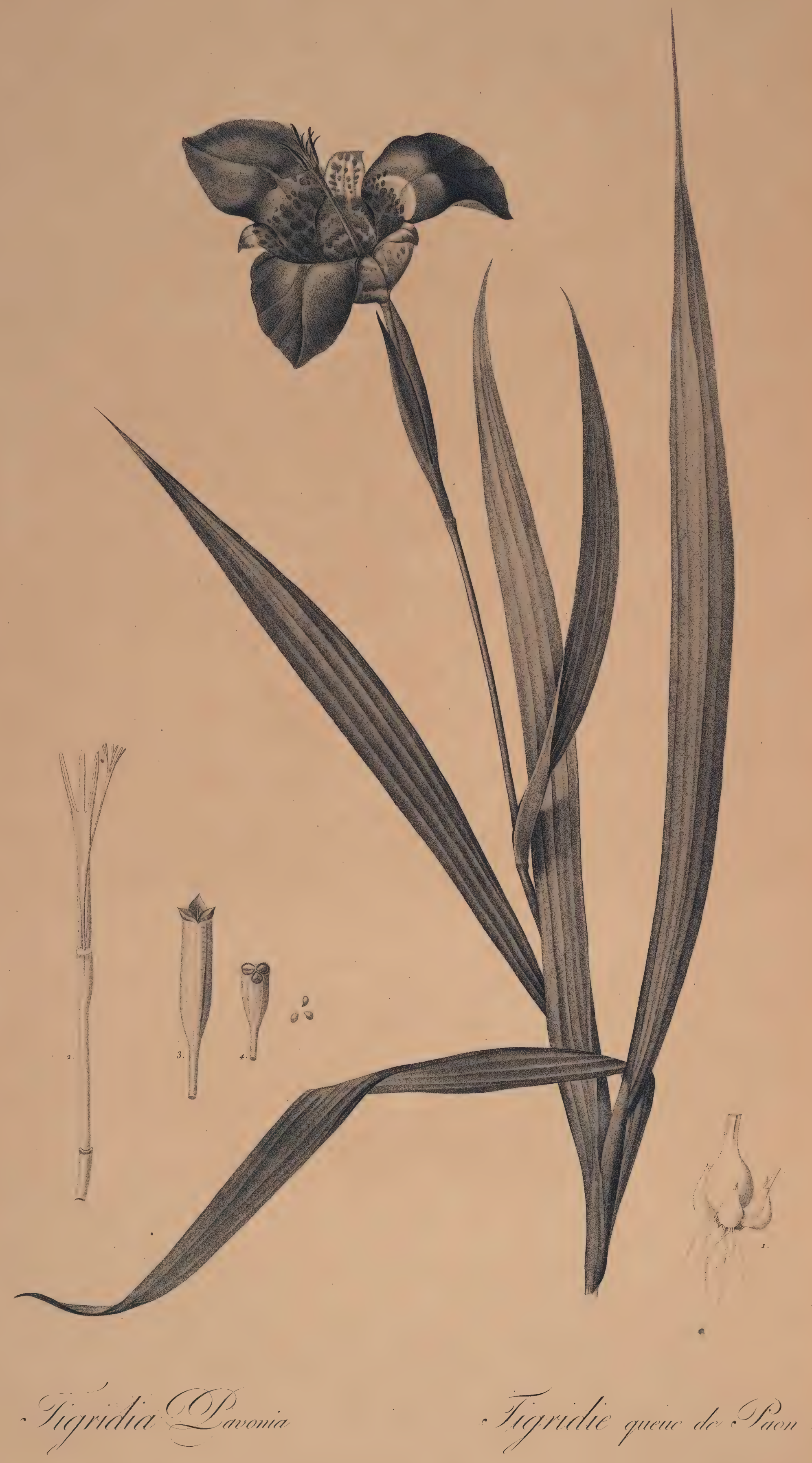




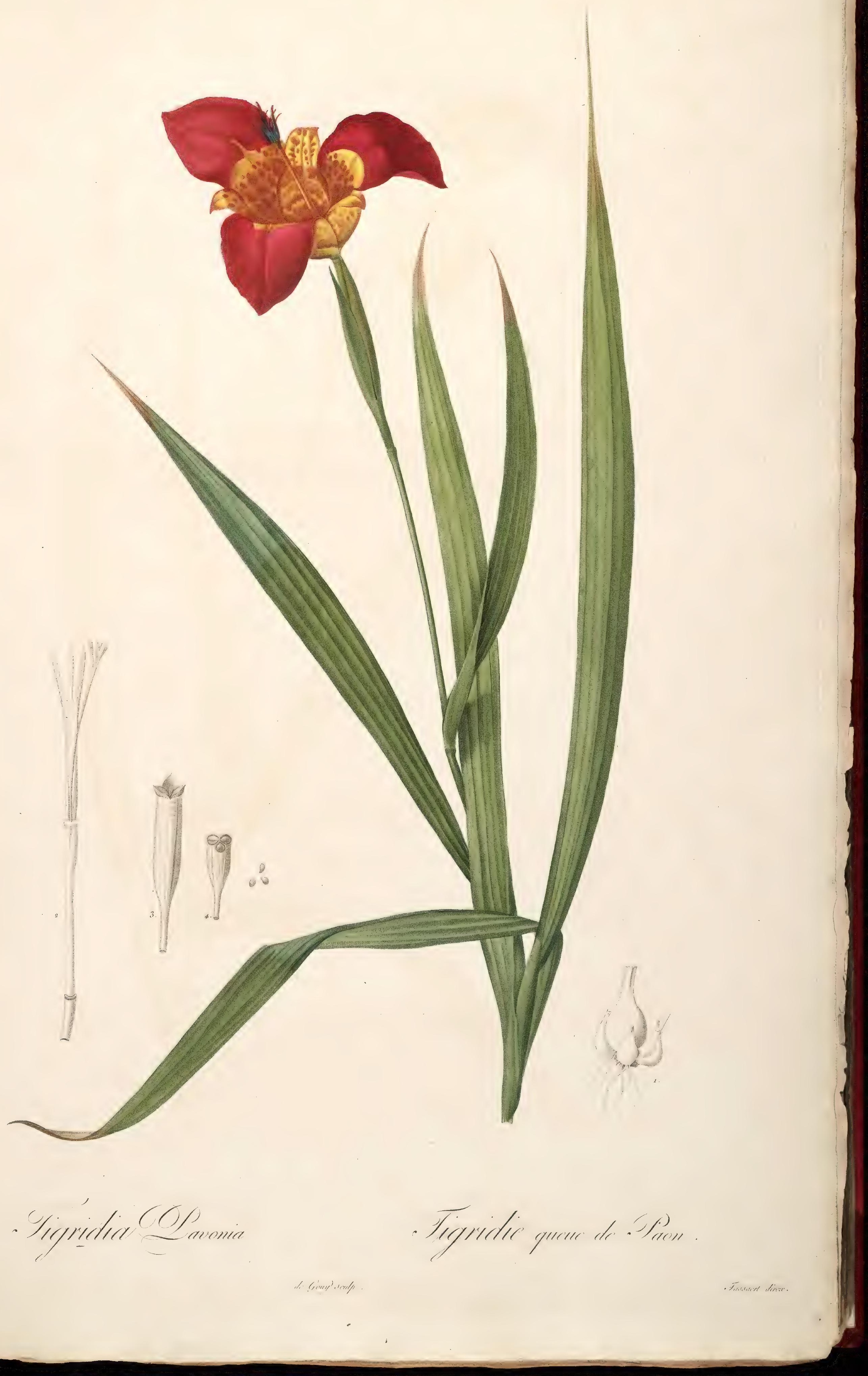




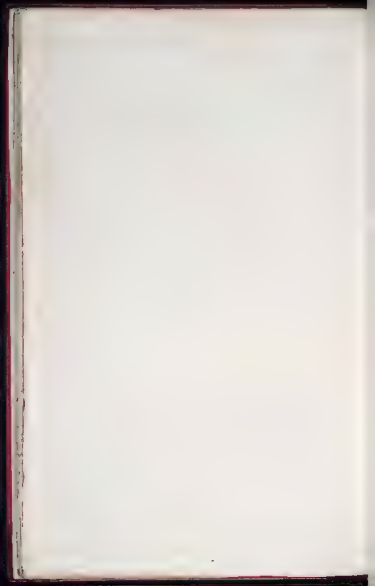




\title{
I.ILUU PONPONIT Y.
}

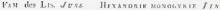

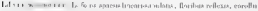

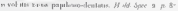

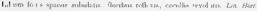

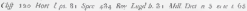

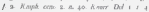

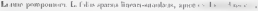

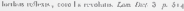

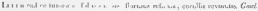

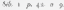

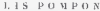

\section{it}

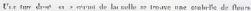

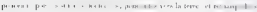

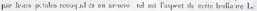
EM:

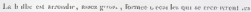

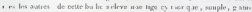

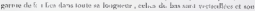

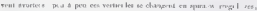

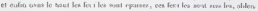

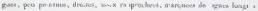

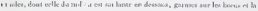

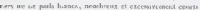

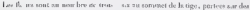

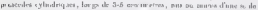

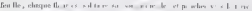

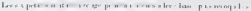

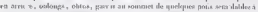

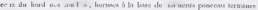

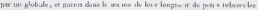

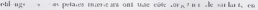
in want : 


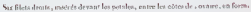

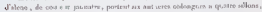

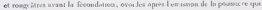

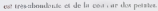

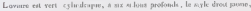

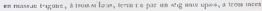

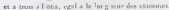

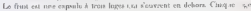

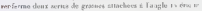

\section{ais $\mathrm{T}$ 앙 it}

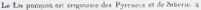

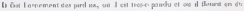

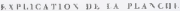

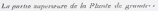

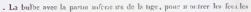
sterbea wrimi lors

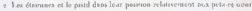
on an bave unem 


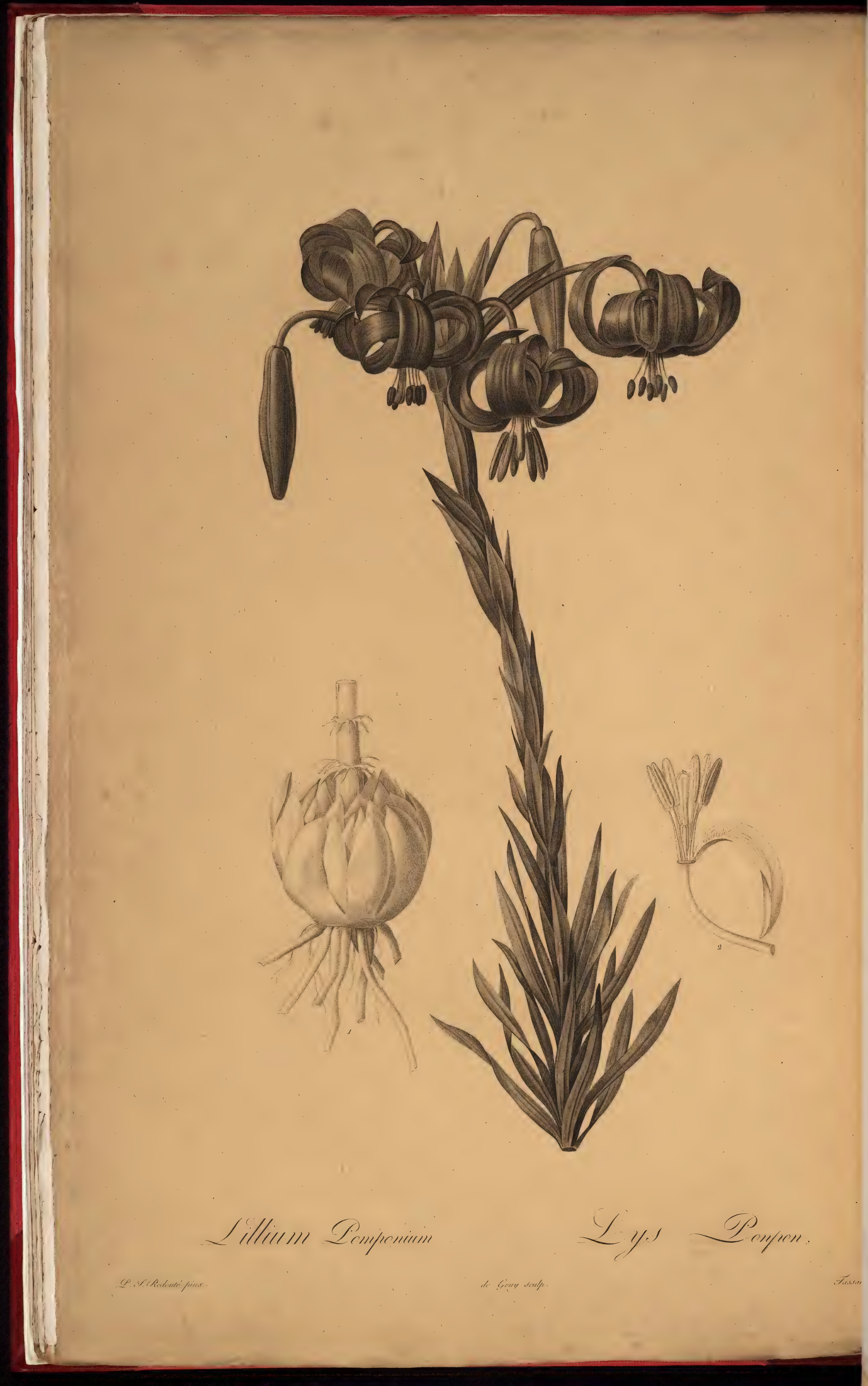




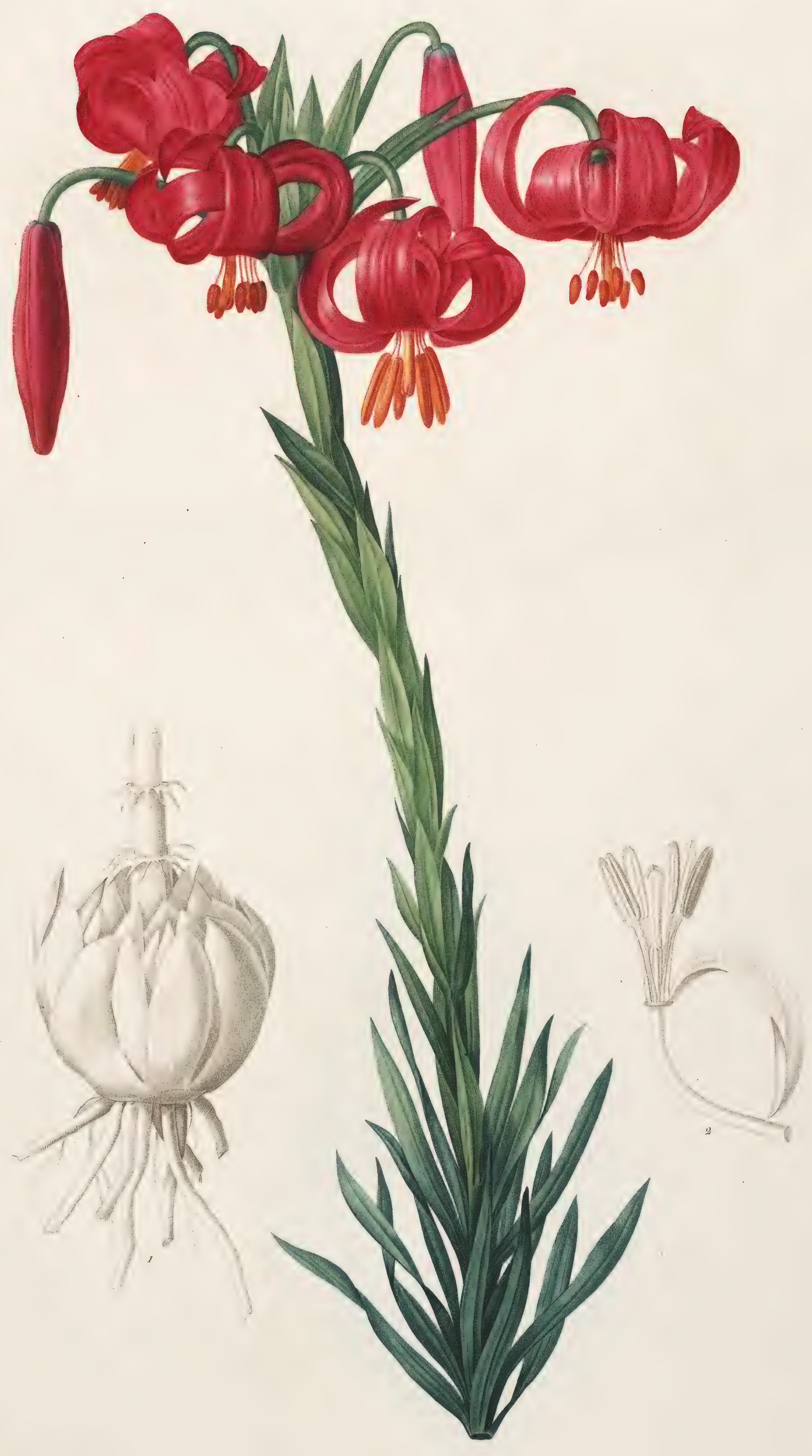

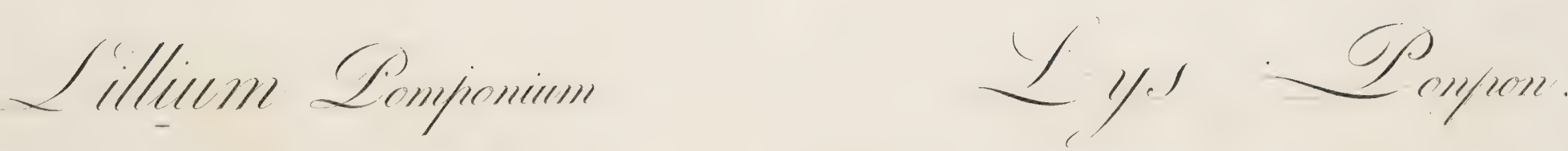




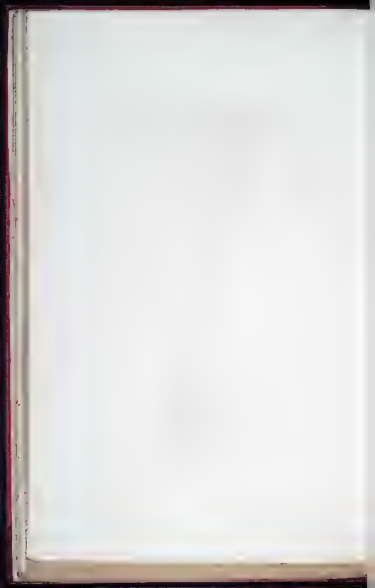




\title{
PIXCRATIU MARITIML H.
}

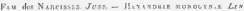

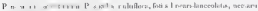

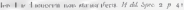

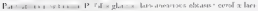

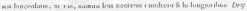
dic i a abs

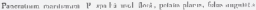

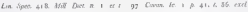
a) 17 is 0

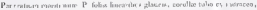

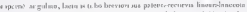

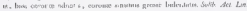

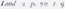

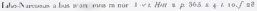
min

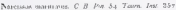

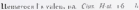

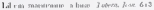

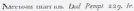

\section{PARCACB NARIL1)}

\section{1) 28 it}

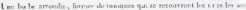

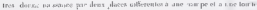

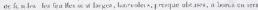

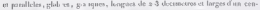

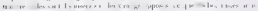
witial one

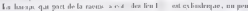

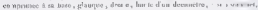

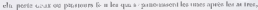

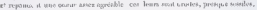

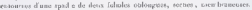




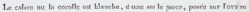

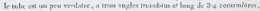

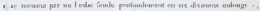

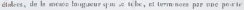

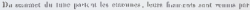

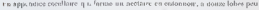

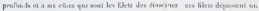

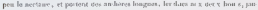

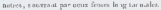

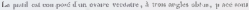

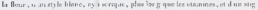

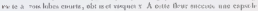

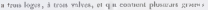

\section{I A T D I E F}

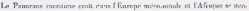

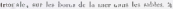

1 bein $\rightarrow$ in: 



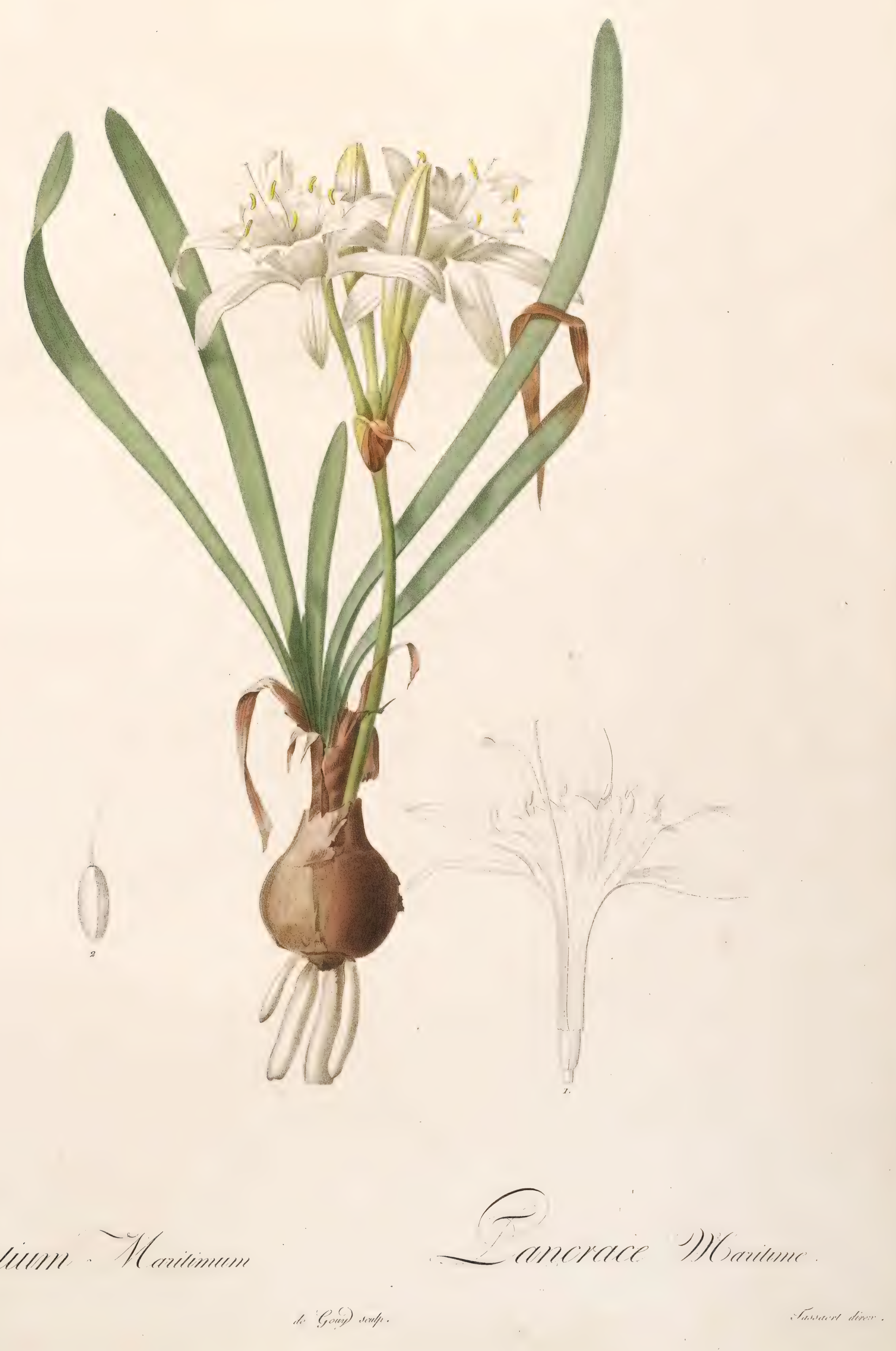




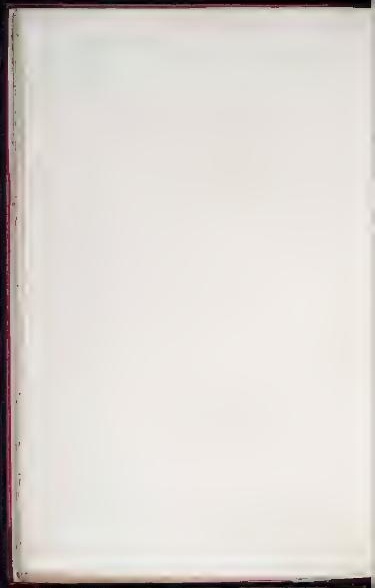




\section{AMARYLLIS REGINE.}

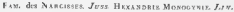

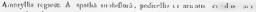

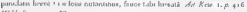
iFill. Bowe a a is

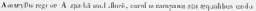

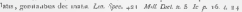

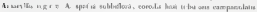

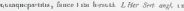

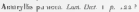

\section{AAARILLIS DE LA AEINT}

\section{DESCRIPTION}

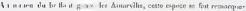

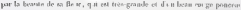

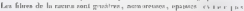

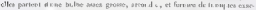

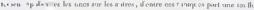

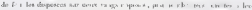

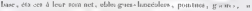

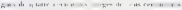

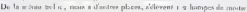

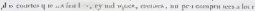

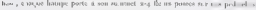

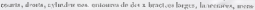

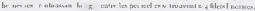

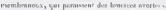

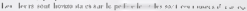

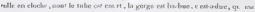

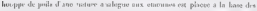

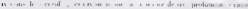

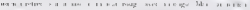

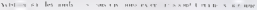

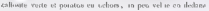




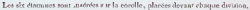

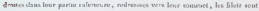

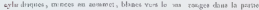

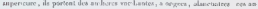

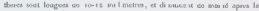
ferumbias:

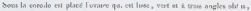

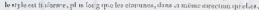

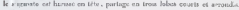

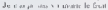

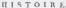

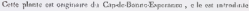

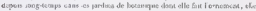

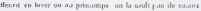

\section{PLECAIIUY DELA PIANEUE}

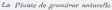

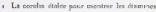

a. Ios paid 



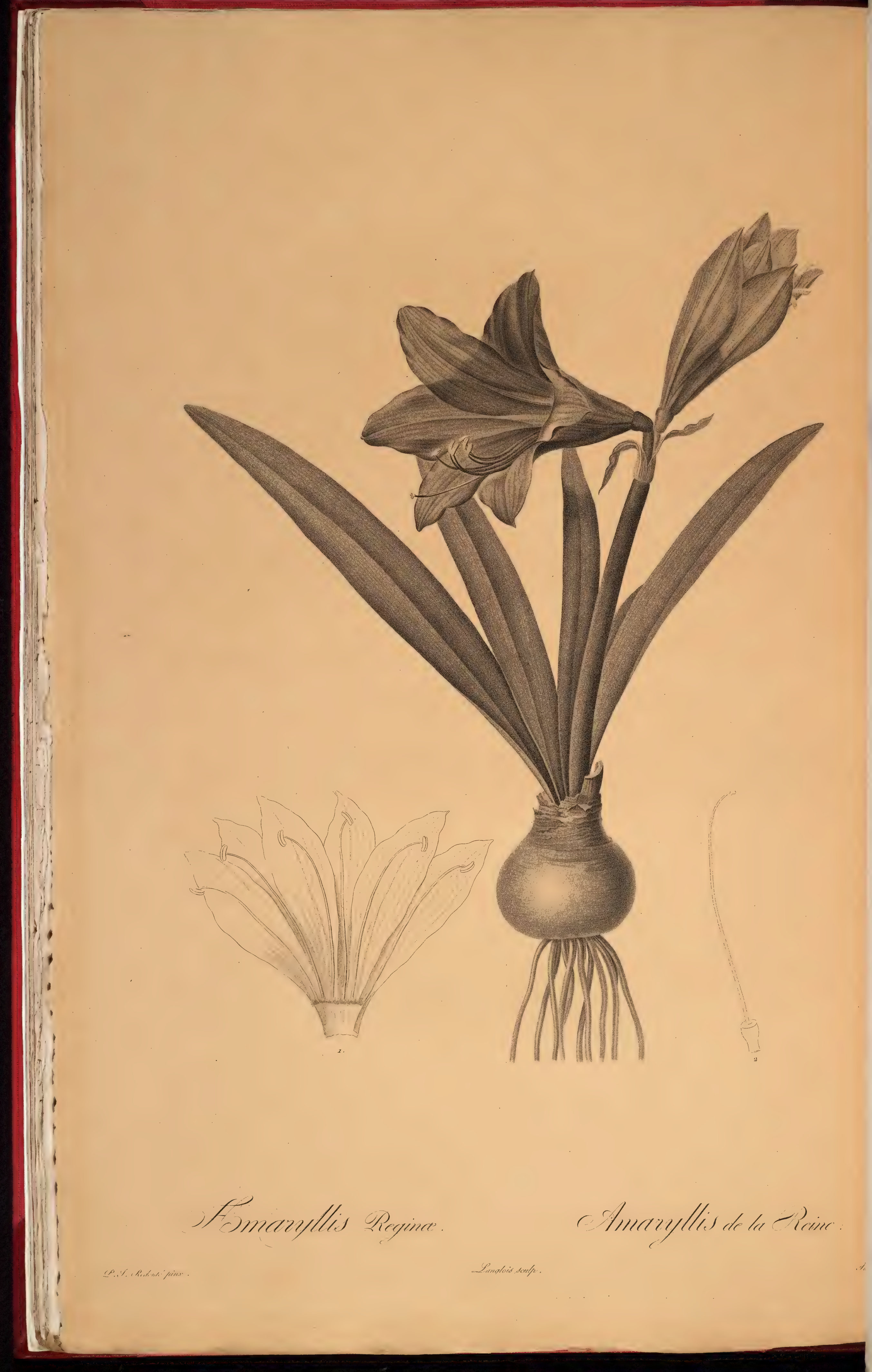




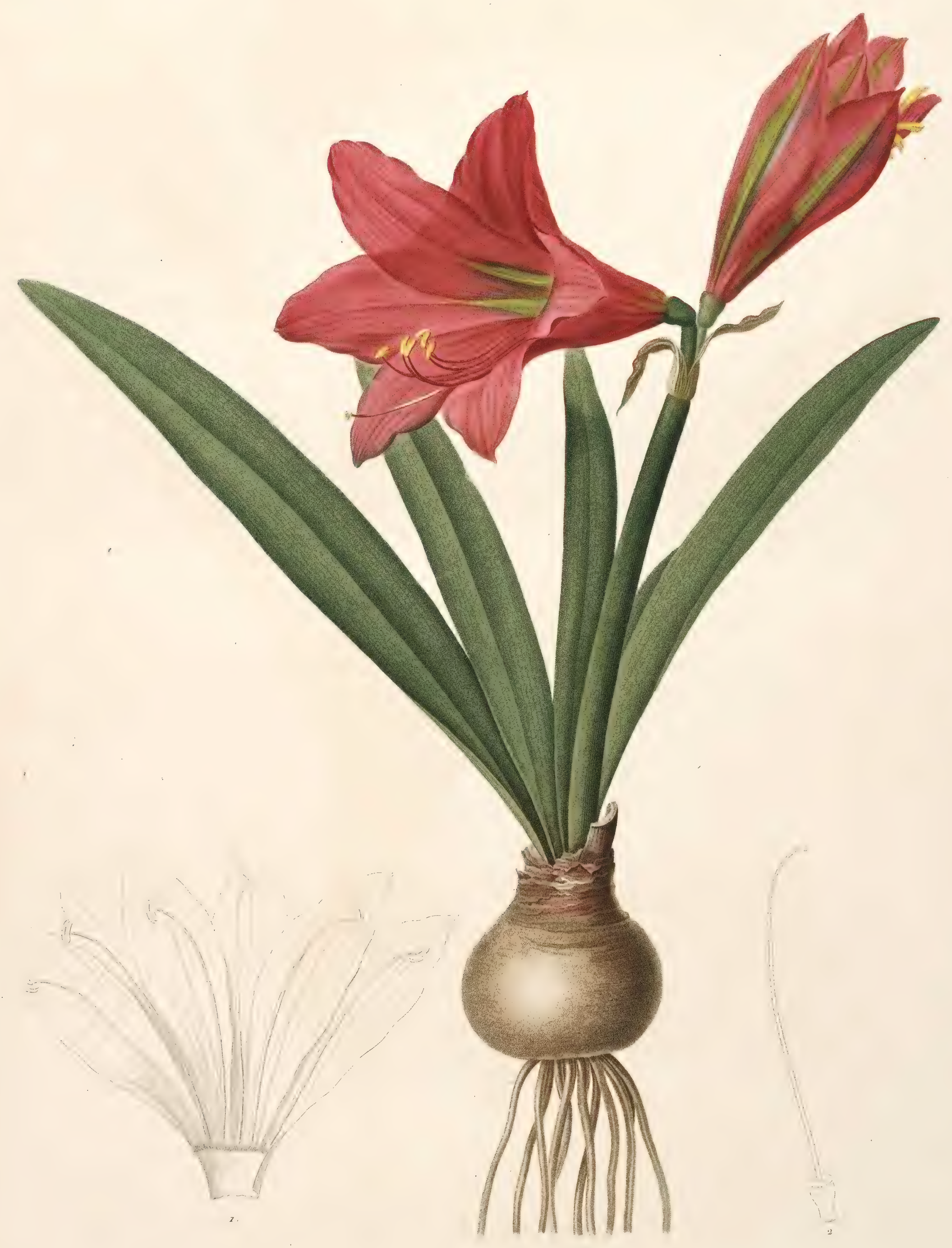

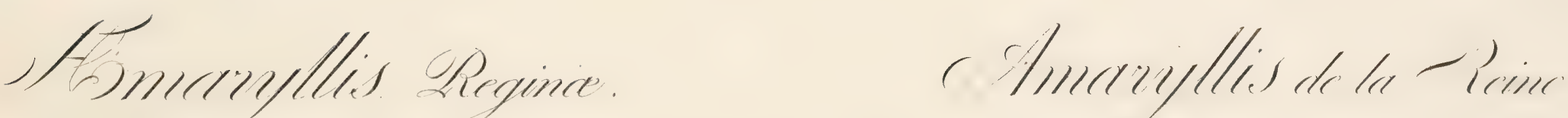




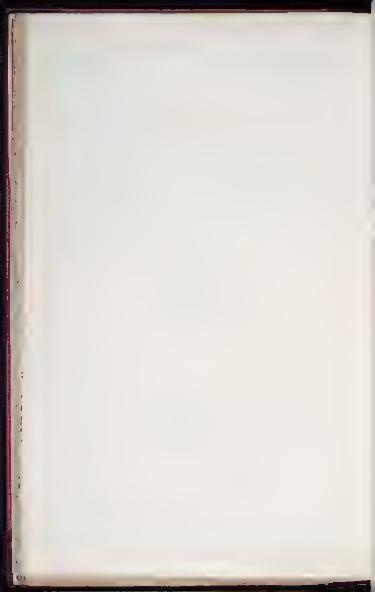




\section{MARYLIS VITTATA.}

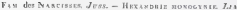

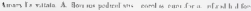

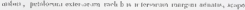

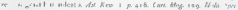
$=\mu-A$

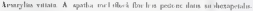

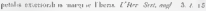

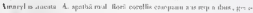

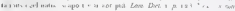

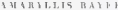

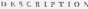

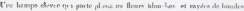

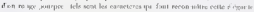

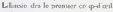

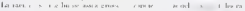

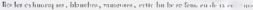

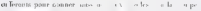

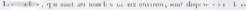

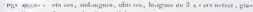

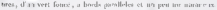

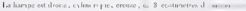

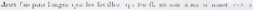

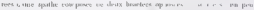

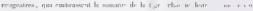

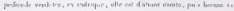

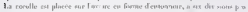

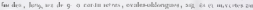

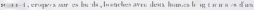
$n \leq$ an

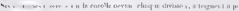

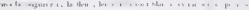

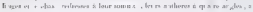




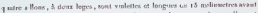

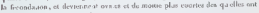

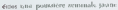

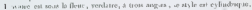

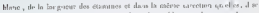

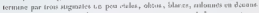

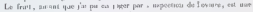

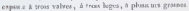

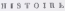

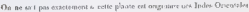

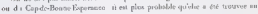
Cang 2

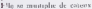

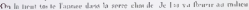

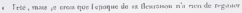

\section{F $X$ PICA TION DE LA PLA V I F}

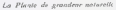

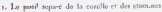





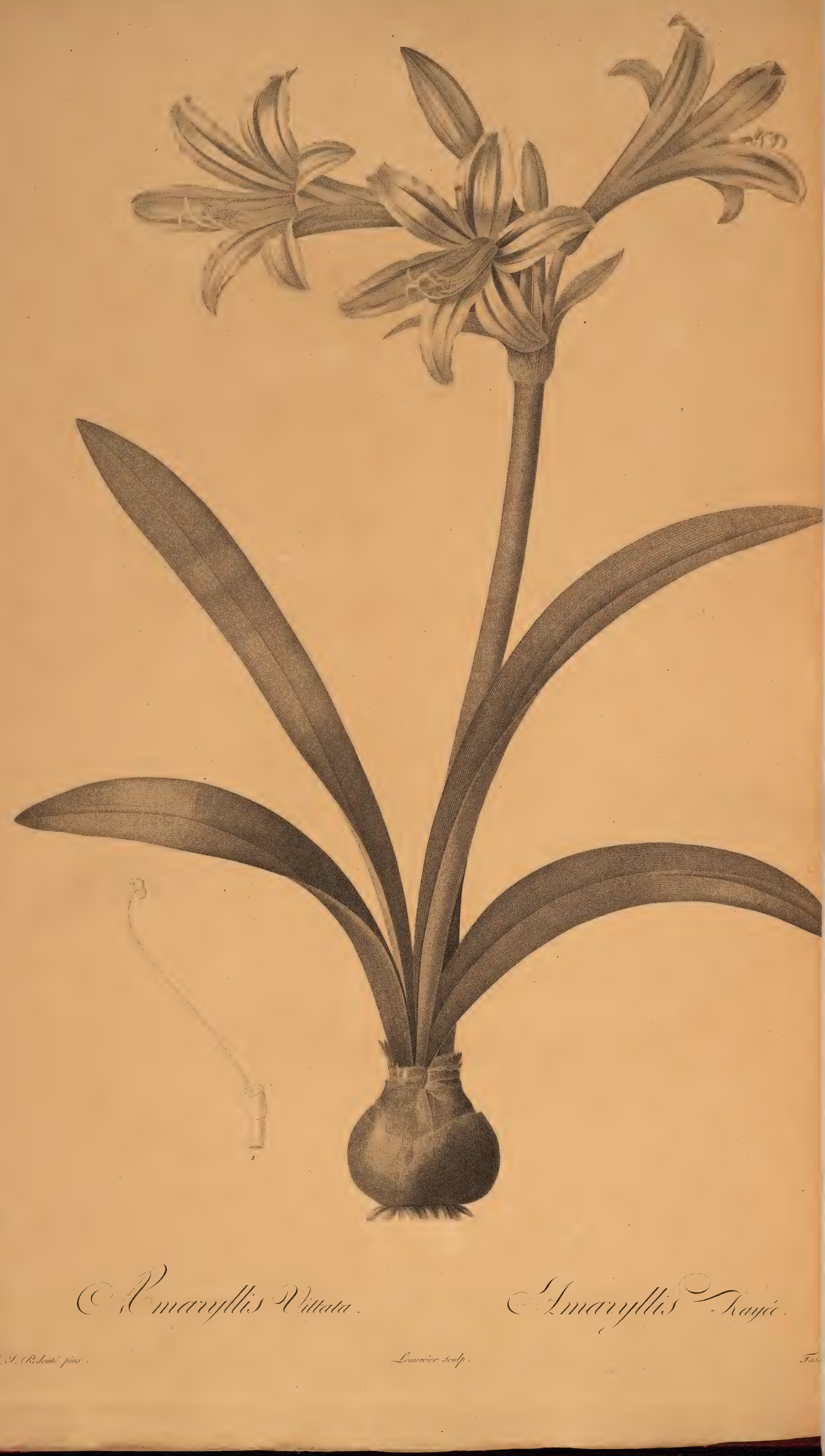




$$
\text { va }
$$




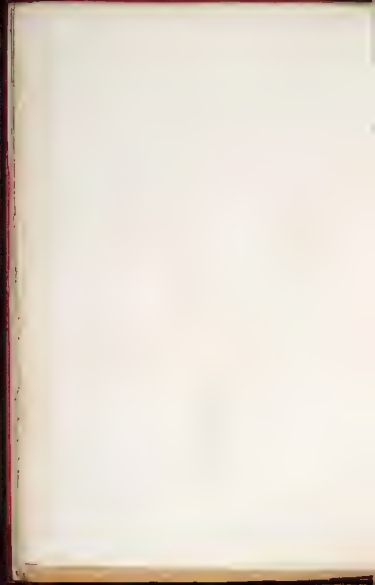




\section{GLADTOLUS MERIAVUS.}

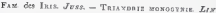

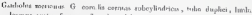

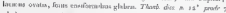

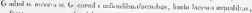

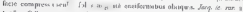

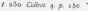

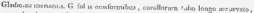

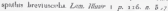

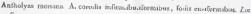
sper it

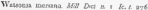

\section{GLIEUL DE NEAIAX}

\section{DES CALPTION}

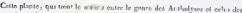

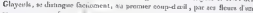

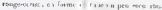

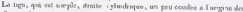

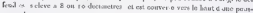
sore bititio

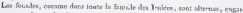

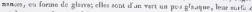

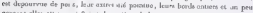

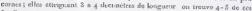

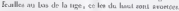

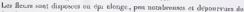

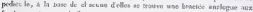

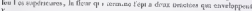
Tetrine

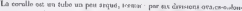

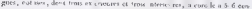

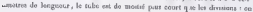




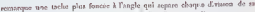

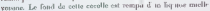

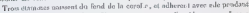

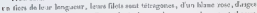

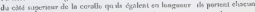

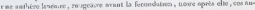

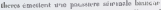

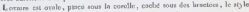

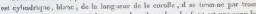

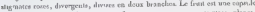

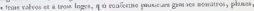

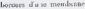

\section{If 1 I T in 8}

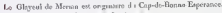

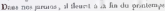

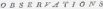

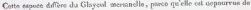
pois

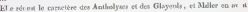

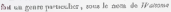

\section{ETPLICATION DE LA PEAACIIE.}

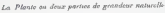

1. La easente osmele prar monifer los fivauces

2 L. part

3 Le fru

4 lis rova de 



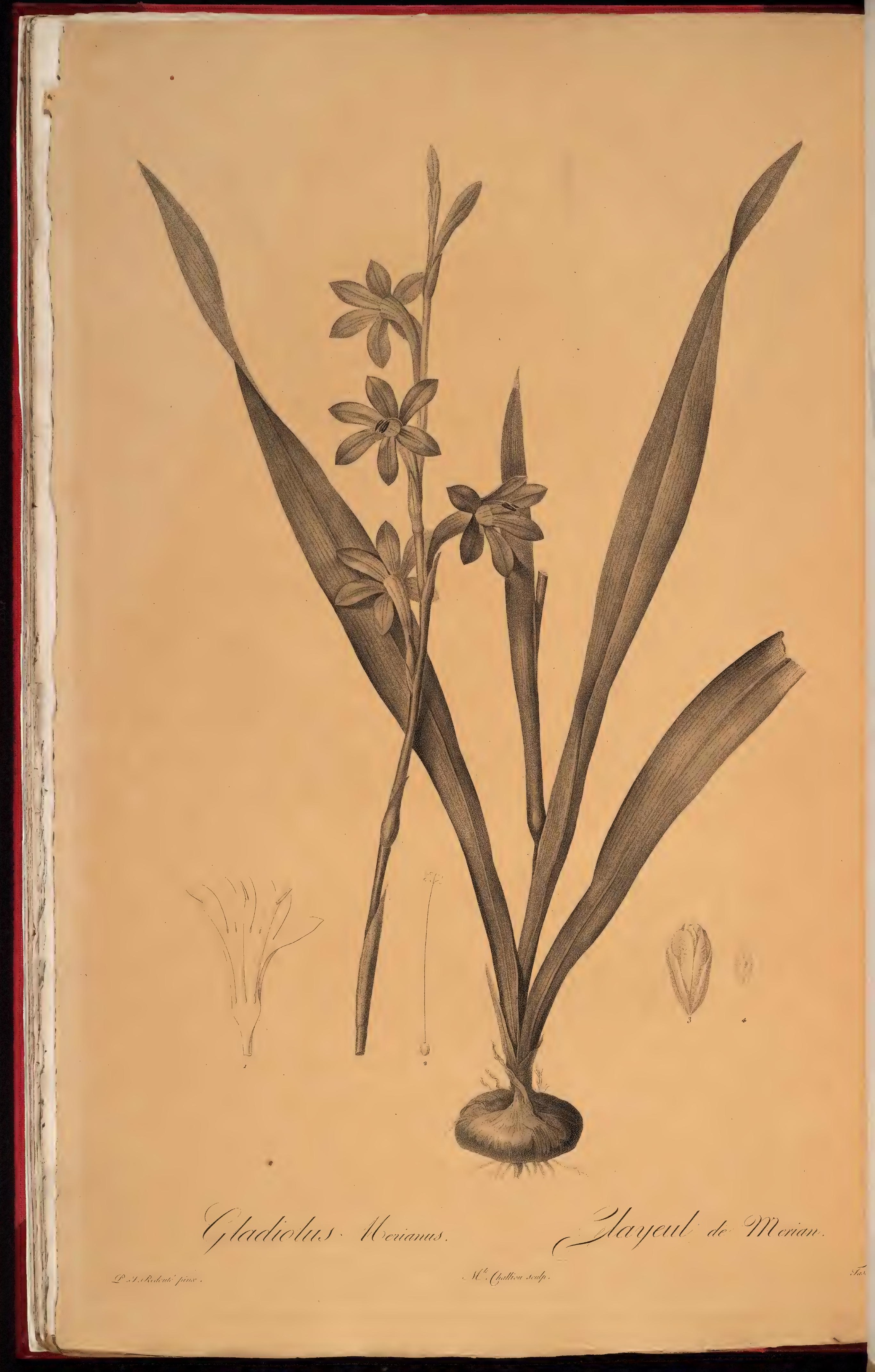




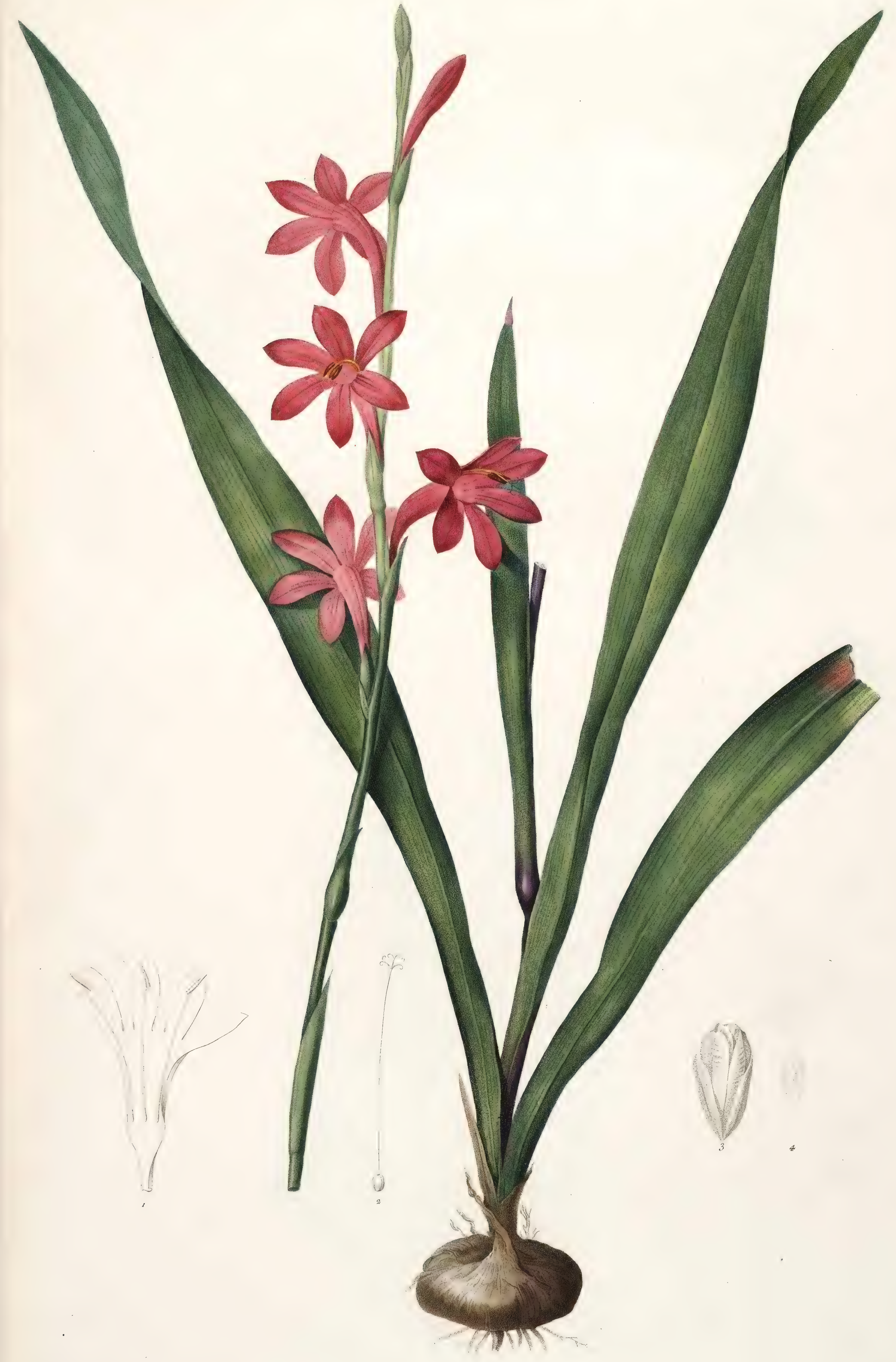

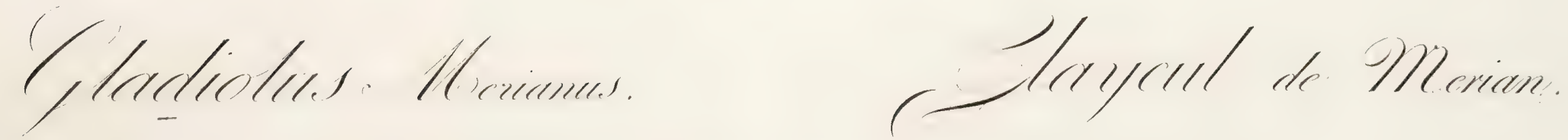




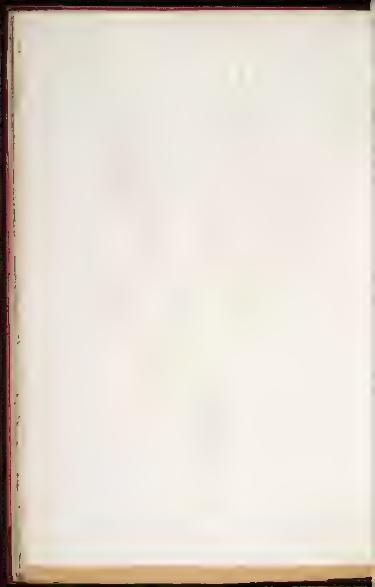




\title{
ANTIOLYZA CUNONIA.
}

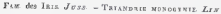

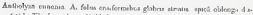

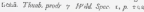

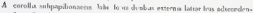

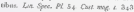

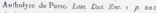

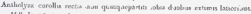
AS

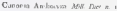

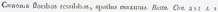

\section{hNTIOLYSE PAPILIONACEL}

\section{DESC R T T I O R}

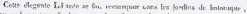

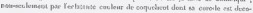

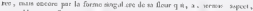
makrablt a sire dijar popr mnacue

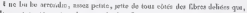

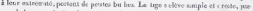

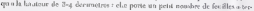

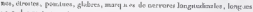
wo g. der melios envimon

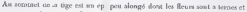

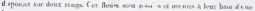

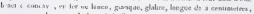

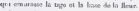

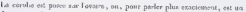

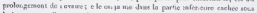

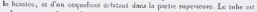

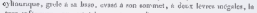

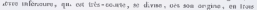

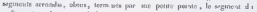

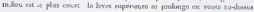




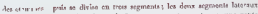

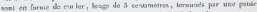

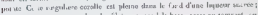

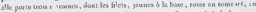

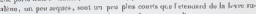

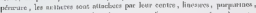

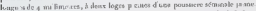

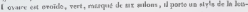

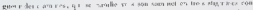

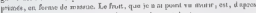

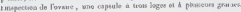

\section{If I 50 I I I h}

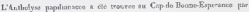

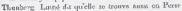

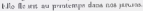

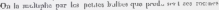

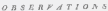

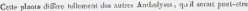

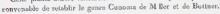

EXPIICATION DE I. $A$ P L A K C I E

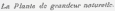
1 Boreve
8. Coculfo atobre
a I ing thancias
4 Poi
s Rxino 



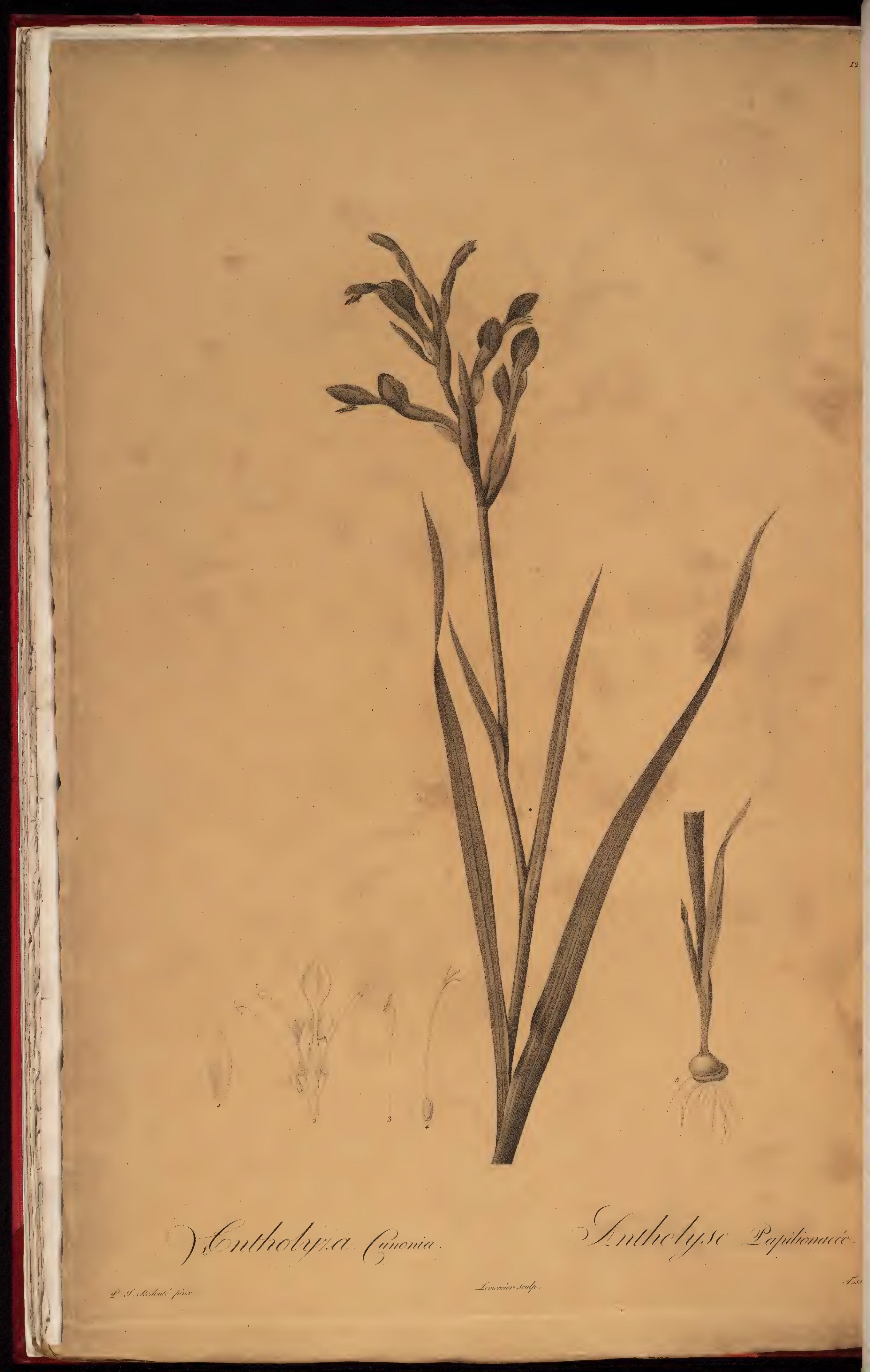




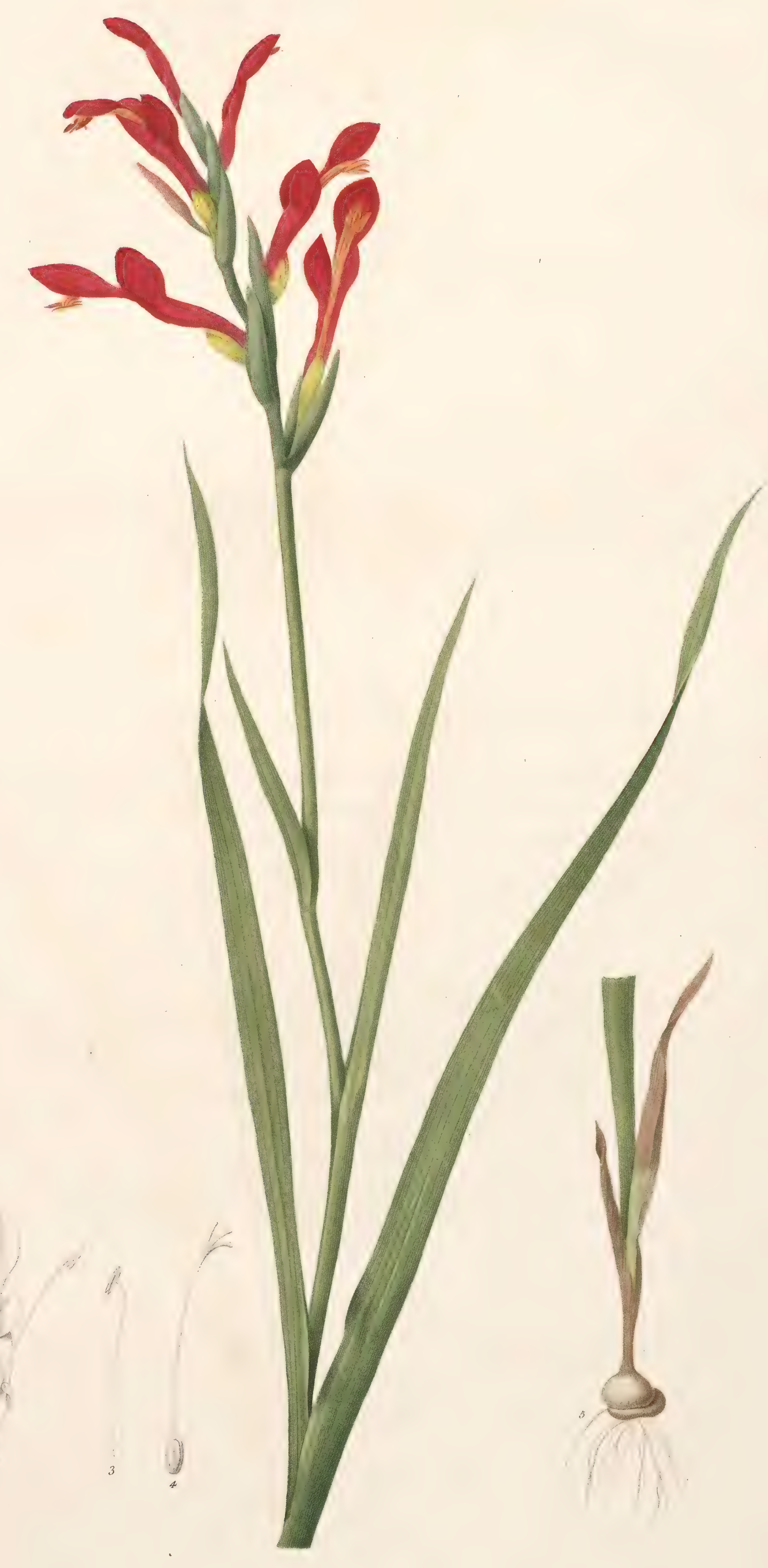

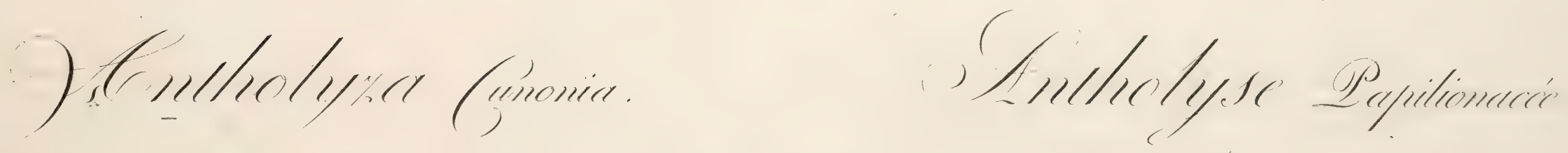




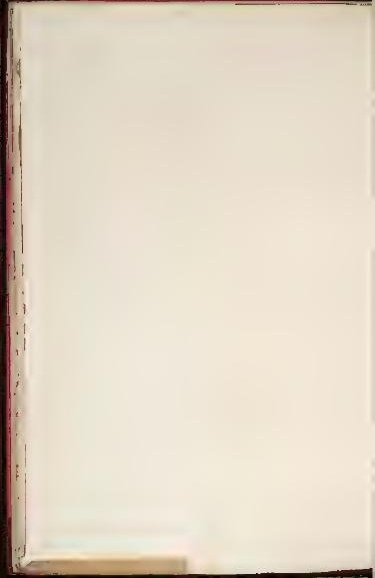




\title{
HELONIAS BULLATA
}

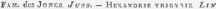

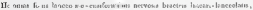

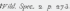

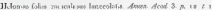

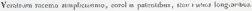

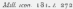

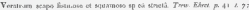

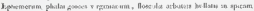

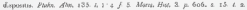
$f=$

\section{HLLONIAS ROSE.}

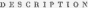

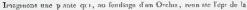

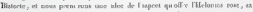

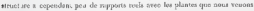
or sthir

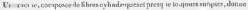

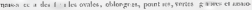

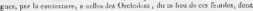

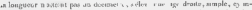

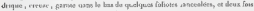

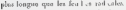

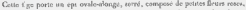

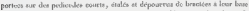

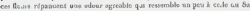
tscii is axar

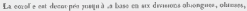

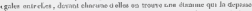

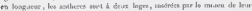

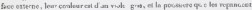

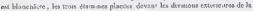

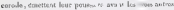




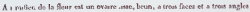

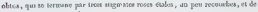

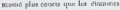

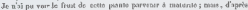

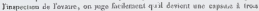

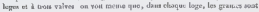

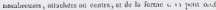

\section{ISTOIR F}

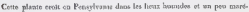
ox. 45.3

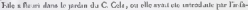

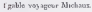

\section{X F I C A TON DE LA FLARCHL}

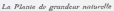

t L. B-ue rue pardreym!

s. Li jlear wae pir-duevere

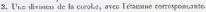

- Los peret, 


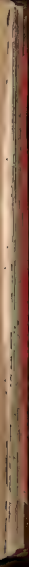




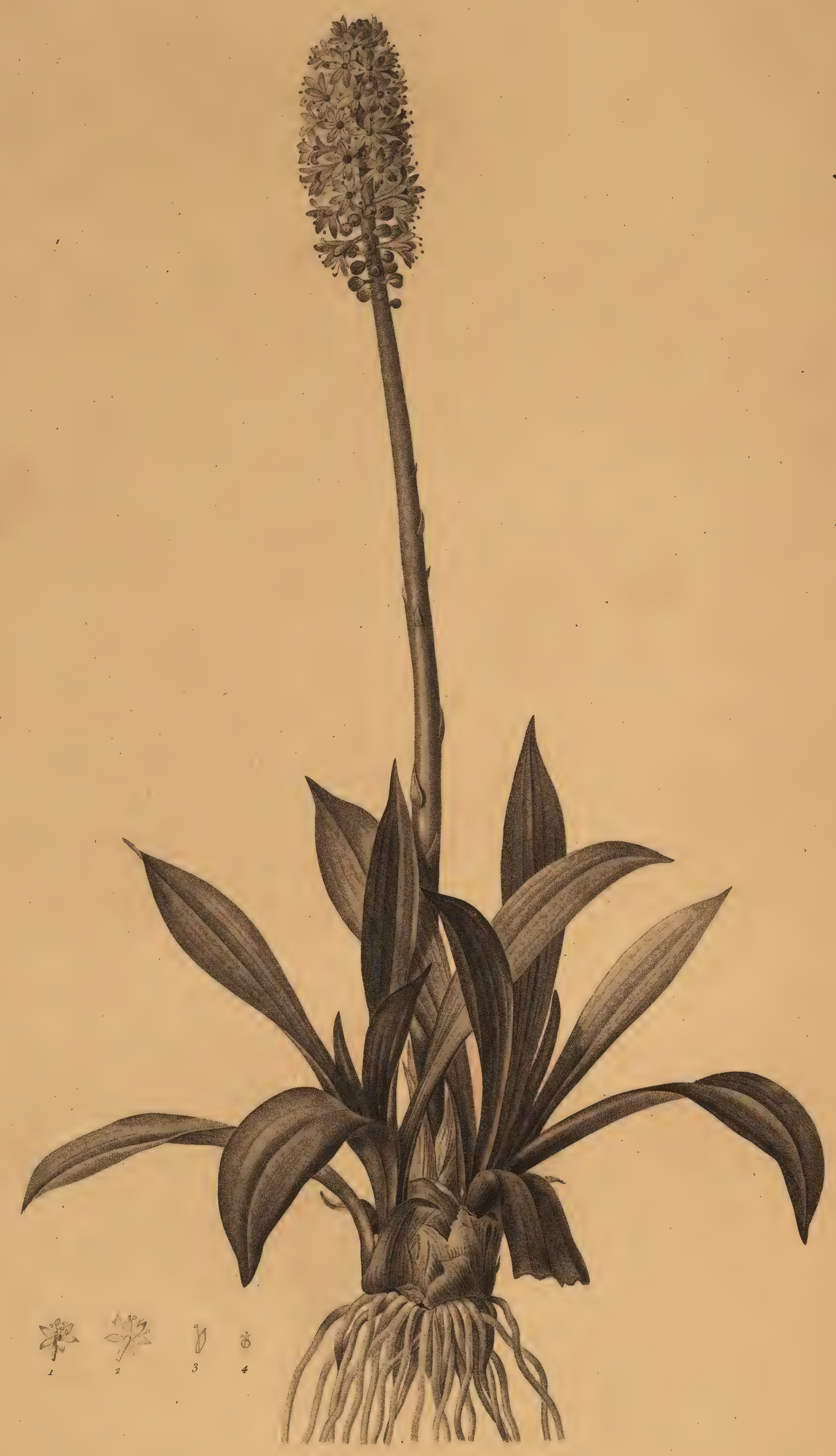

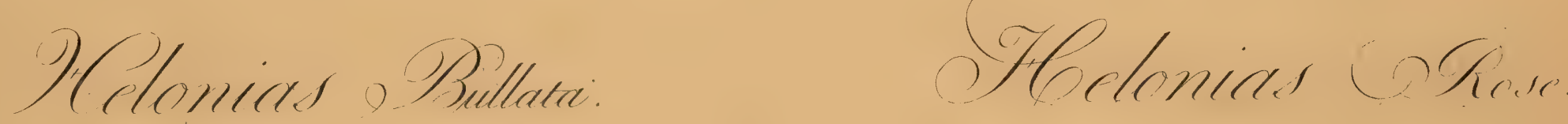





\title{
IIY ICINTIIS A METIYYSTINUS.
}

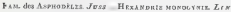

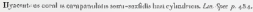

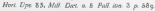

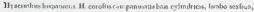

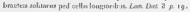

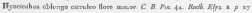

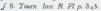

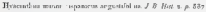

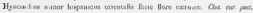
ie fid id

\section{IACIOTHE A UETHYSTE}

\section{DESETIPTION}

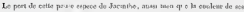

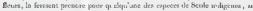

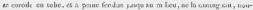

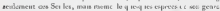

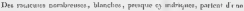

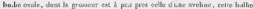

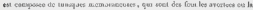
bive ratuie doe For il wh

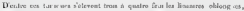

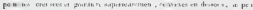

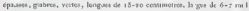

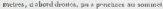

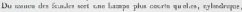

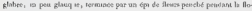

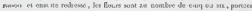

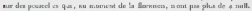

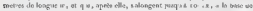

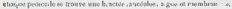

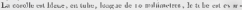

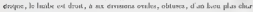

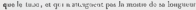




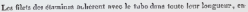

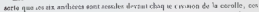

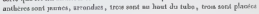
at: calved de so loopprar

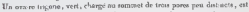

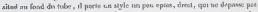

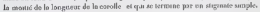

In corulle as trombo por apres a Ronisoe, mour dile reccairse une capude

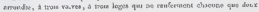
gen ate

\section{แI 1 T O I I I}

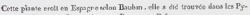

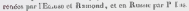

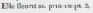

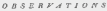

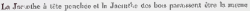

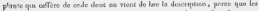

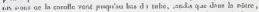

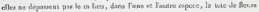

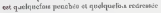

\section{TXPLIOATION DE LA PIAXEHE}

to Mhonst do gendelcur milurets

1. Ia flenr en ica.

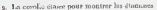

+10 . 14.1 .

4. La sapoule

5 L a gravar. 





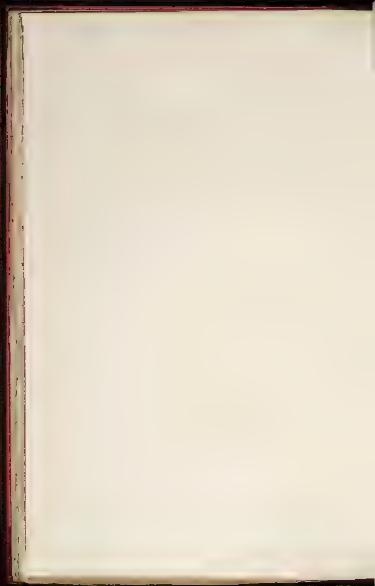




\title{
IIEMEROCALLIS FLAVA.
}

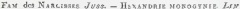

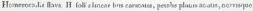

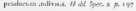

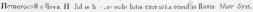

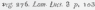

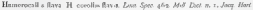

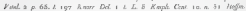
Derax $1=$ S

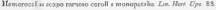

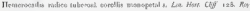
rowath efi

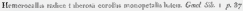

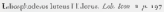

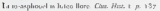

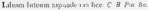

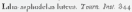

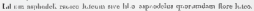

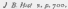

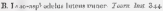

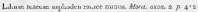

\section{HEREROCALLF JAVY}

\section{DEGCRIFTIOA}

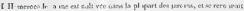

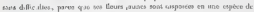
enrotio

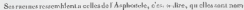

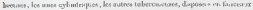

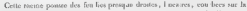

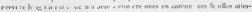

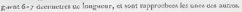




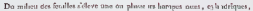

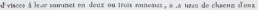

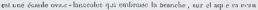

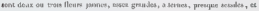

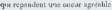

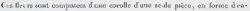

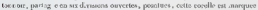

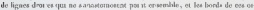

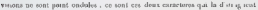
on tespros arrante.

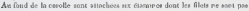

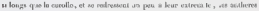
vort of long ies et racillinaks

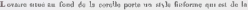

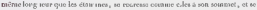
ter wine por wo wisinate simple

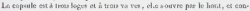
fient pasaçur pronge ncerea el andules

\section{1 i O I I E}

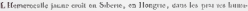
alin. 5 .

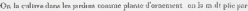
2. acparatien des netses

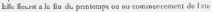





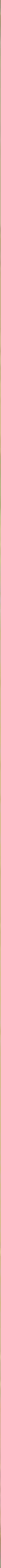




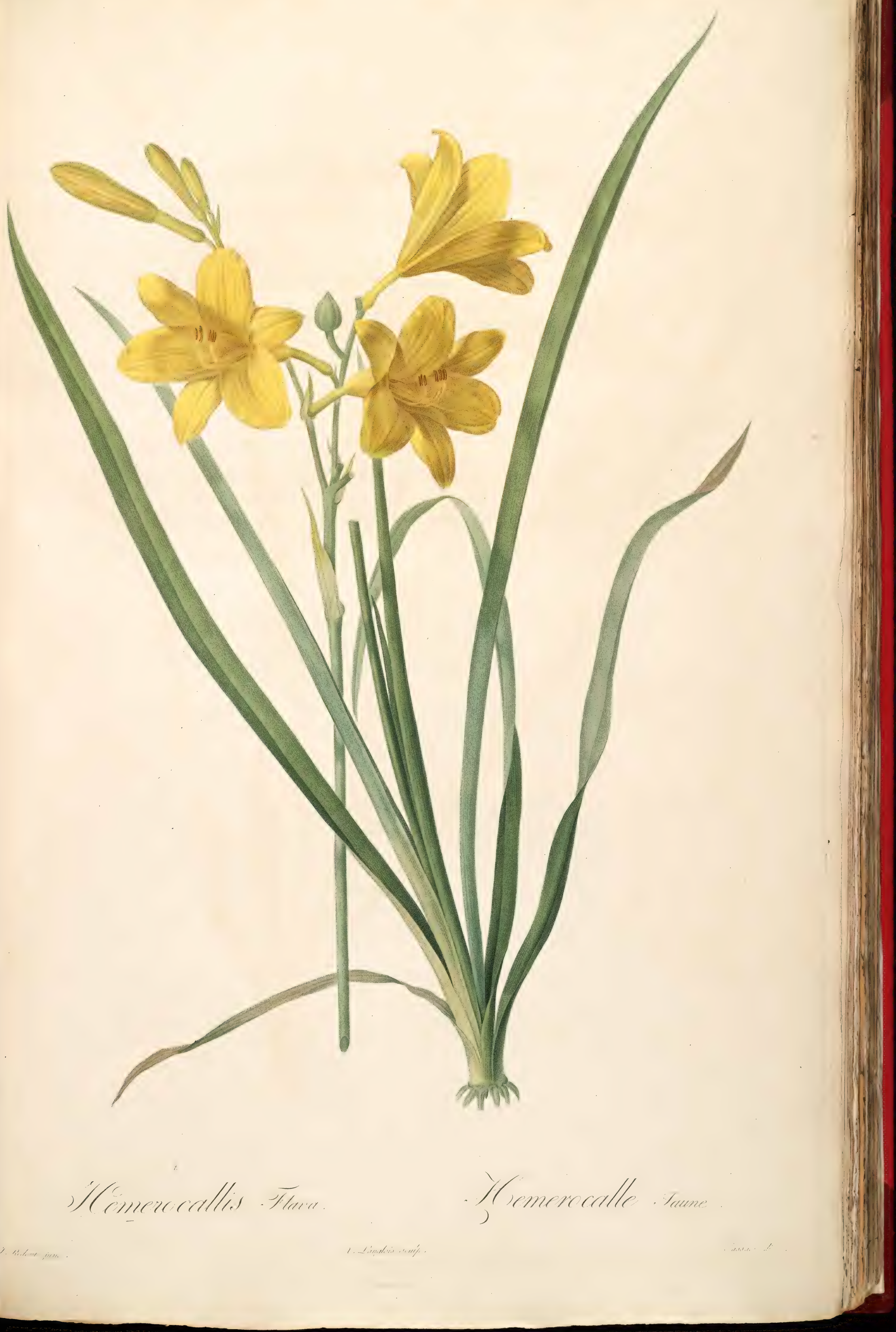





\title{
II E HEROCALLIS FULVA.
}

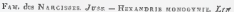

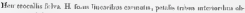

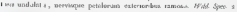
f. 187

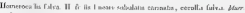

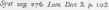

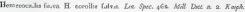
cant 7 of 8 ,

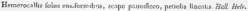
use. pis ras:

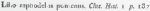

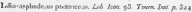

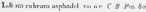

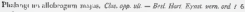
f.

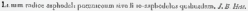
a fai joi

\section{IIE NEROCALLE FAUVE.}

\section{fESCRIPTIOF.}

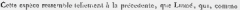

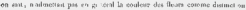

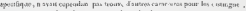

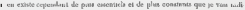
auer imdishogitsoent.

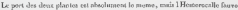

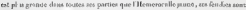

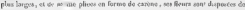

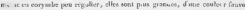

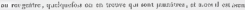

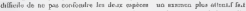

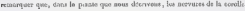




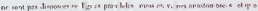

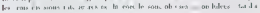

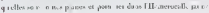

\section{กแร $10121 \mathrm{R}$}

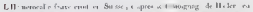

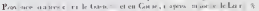

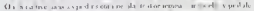

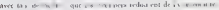

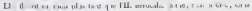
le rither at: 


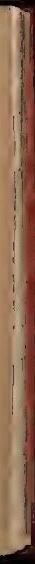




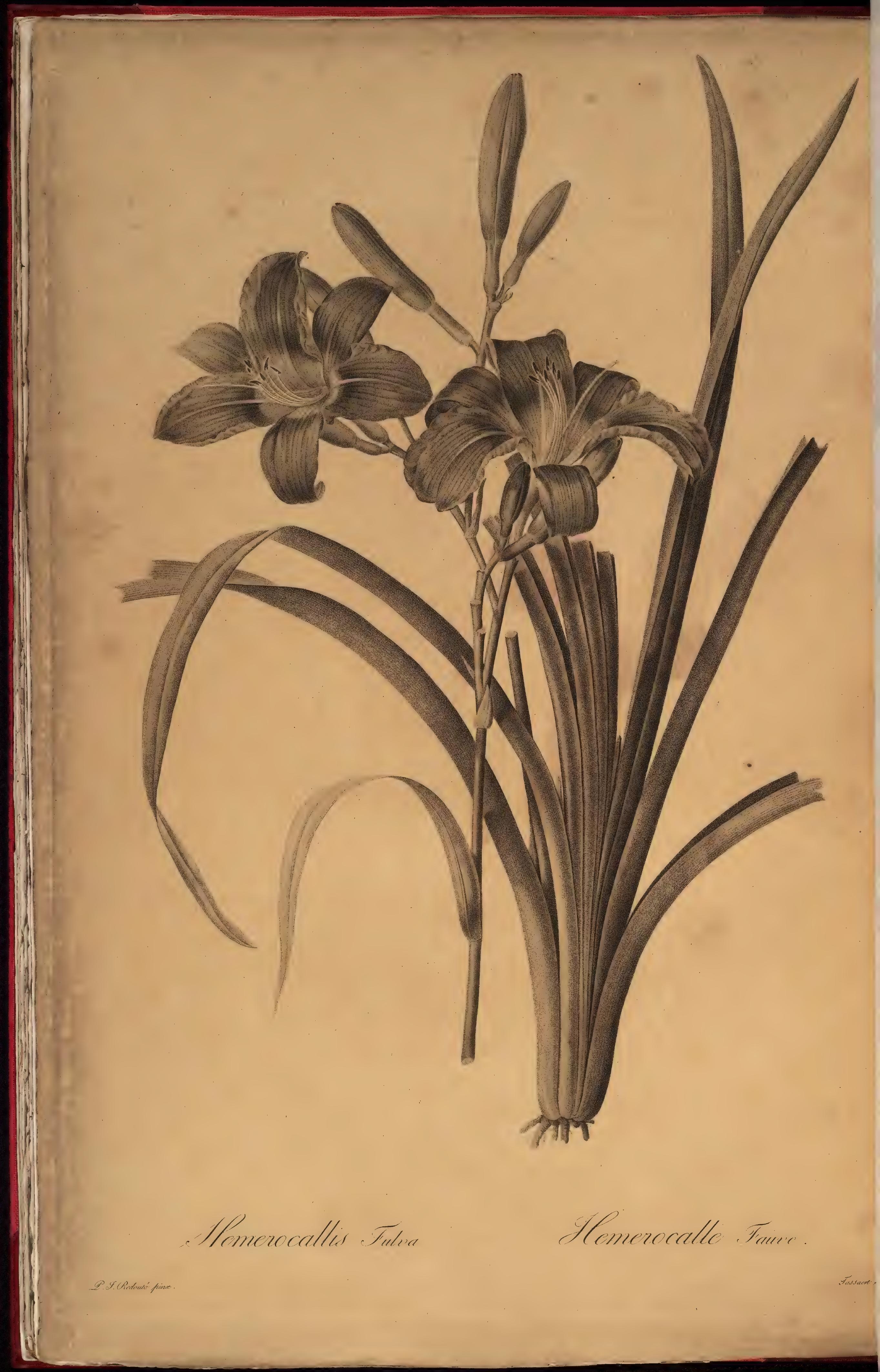




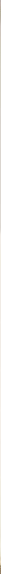




\section{NARCISSUS TAZETT.}

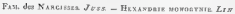

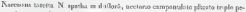

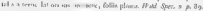

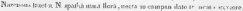

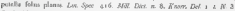

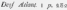

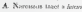

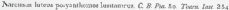

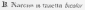

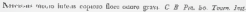

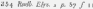

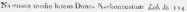

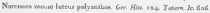

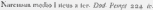

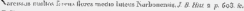
C. Xarnices eamas dobet

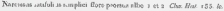

\section{XAIRCISSE A PLUSIELIS FLEURS}

\section{DESC intetor}

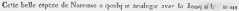

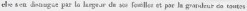
serporit.

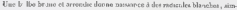

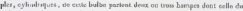

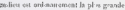

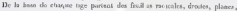

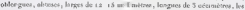

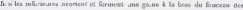

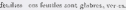

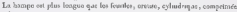
an bies 


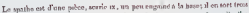

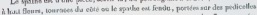

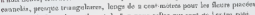

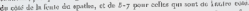

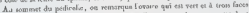

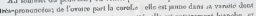

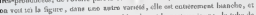

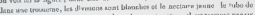

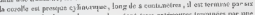

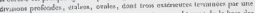

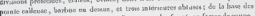

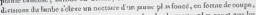

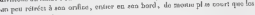
diviuaas du vembe

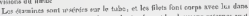

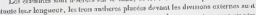

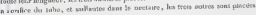
plus bos es renkermere dsas is rabo

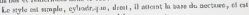

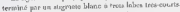

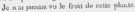

\section{E I 8 T OI I E}

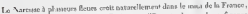

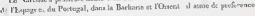
let pritices liuaudes

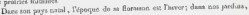

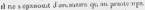

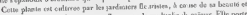

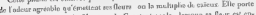

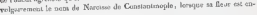
arrevest jawn

\section{EXELICATION DE LA DLAXCII}

\section{La Nhanbe da gndindour nassinfite.}

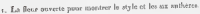




$$
y
$$




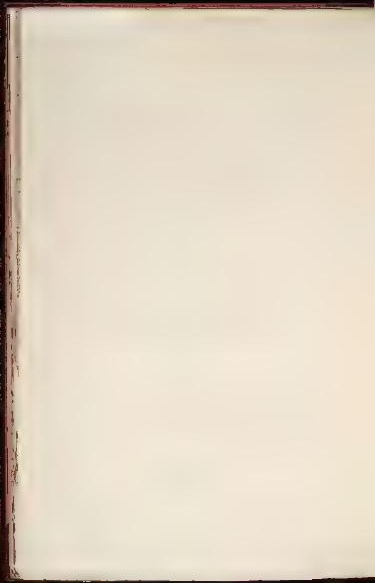




\section{IRIS SUZIANA.}

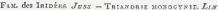

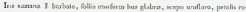

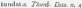

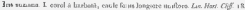

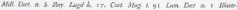
n $\mathrm{at}_{3}$

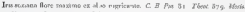

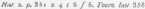

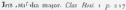

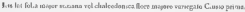
10 thist: 5 . 0 is

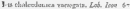

\section{I I S DE B I I I}

\section{E 5 CM IPT IO N}

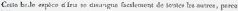

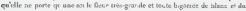
prarpo aurue

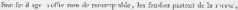

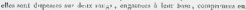

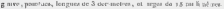

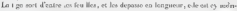

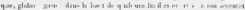

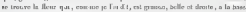

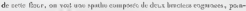

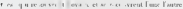

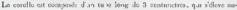

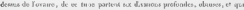

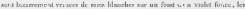

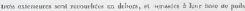

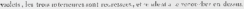

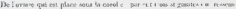

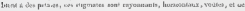




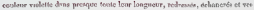

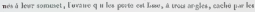
bencker.

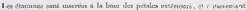

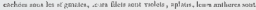

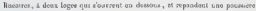
bliminge

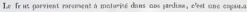

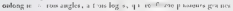

\section{9 T O I I R}

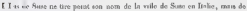

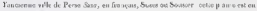

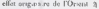

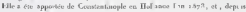

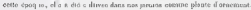

Lie llourat it ha he priabiagy. 



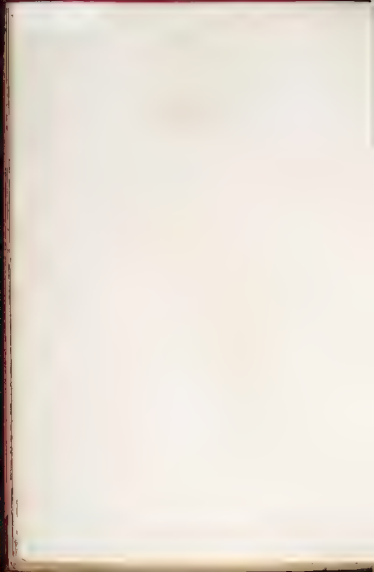




\title{
CYPRIPEDIUM CALCEOLES.
}

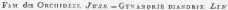

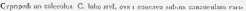

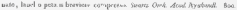
A. 5 . 1

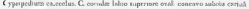

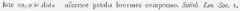
P. 77 \&. 2

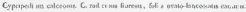

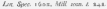

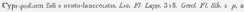
2. 1

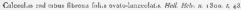

Caleval is its $x$ a

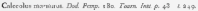

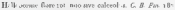

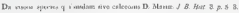

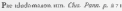

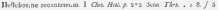

\section{SA BOI DES ALPES.}

\section{IS ESCAT PTIOR.}

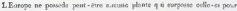

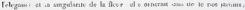

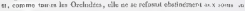
up I ve $x$

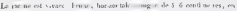

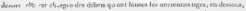

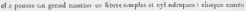

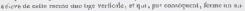

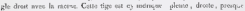

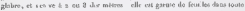

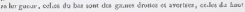




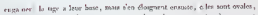

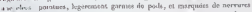

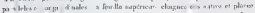

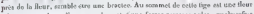

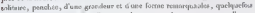

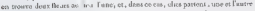

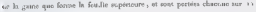
pidarlis.

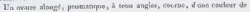

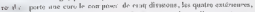

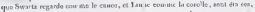

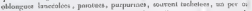

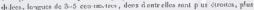

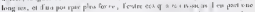

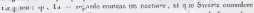

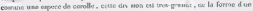

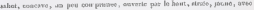
чusthasa vesnes parparines

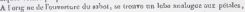

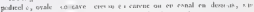

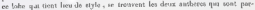

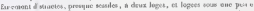

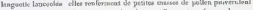

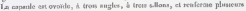

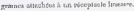

\section{T. TO I I I}

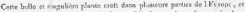

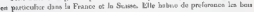
wes exer sowes at

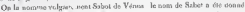

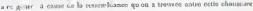

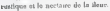





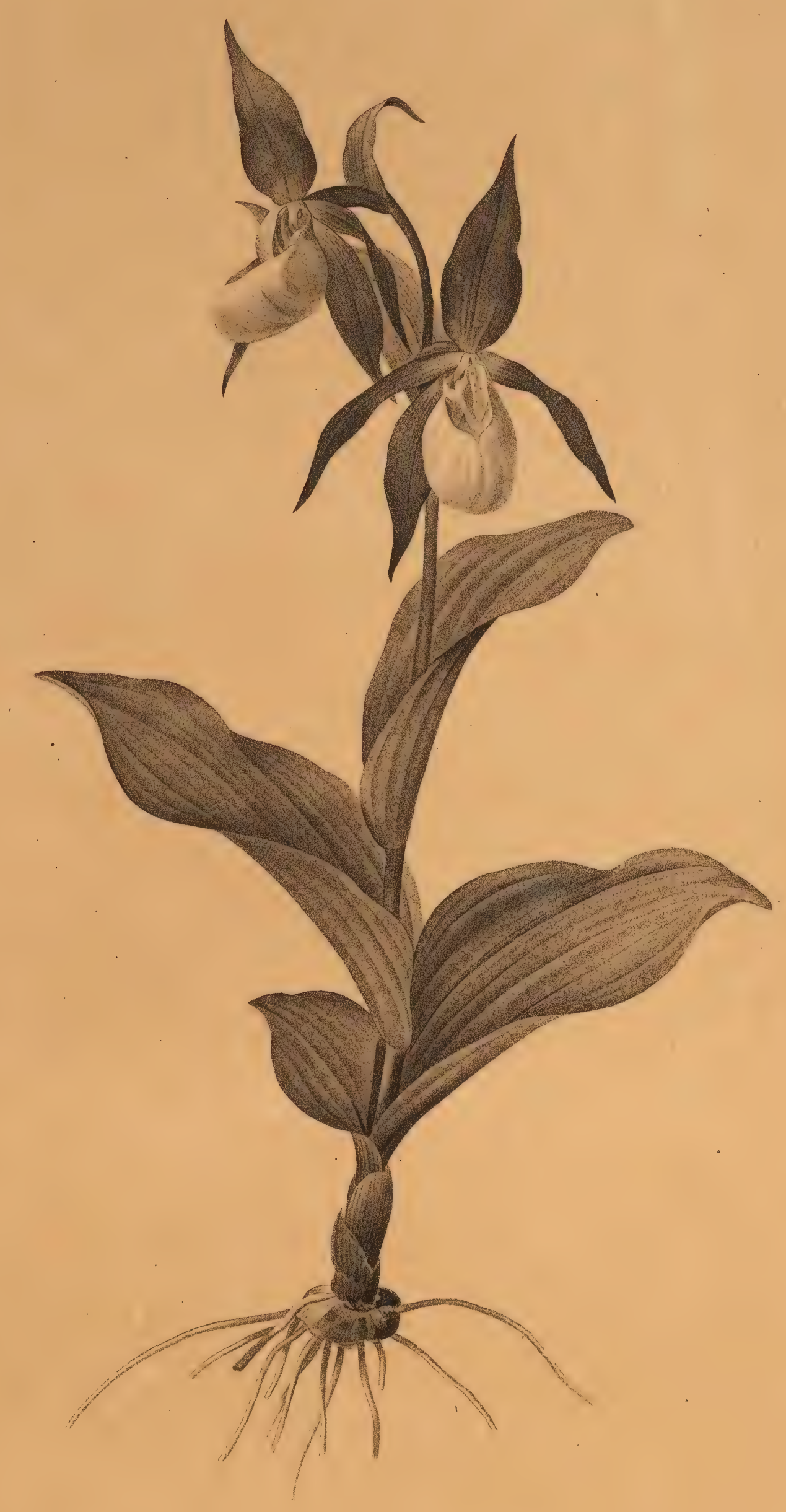

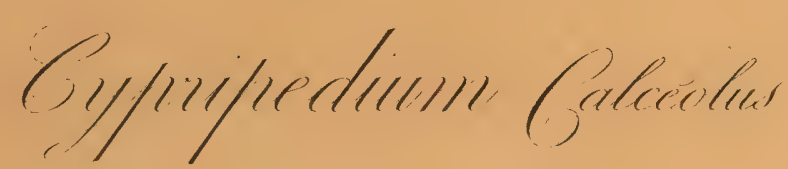

- Cabor cides Colfues. 


$$
\text { 草 }
$$




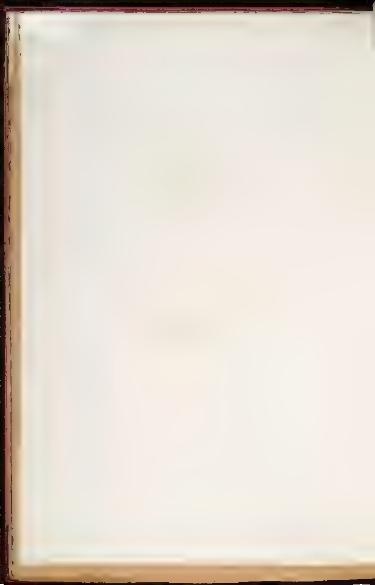




\title{
CYPRIPEDIUM FLAVESCENS.
}

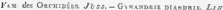

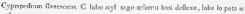

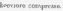

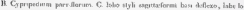

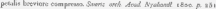

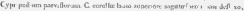

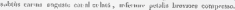

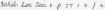

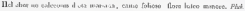

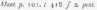

\section{S.MOOF \& FLFT TS JALE}

\section{DISCA I PT T}

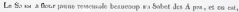

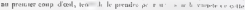

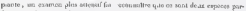

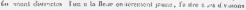

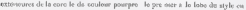

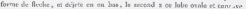

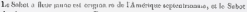
Jes bepes in ac at we qu cu lin ape.

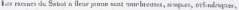

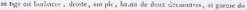

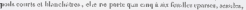

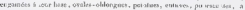

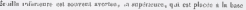

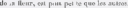

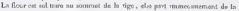

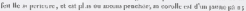

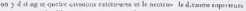

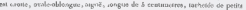

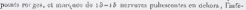

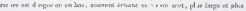

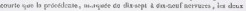




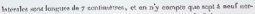

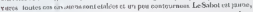

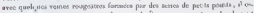

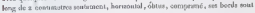
rev ing in ustien:

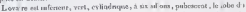

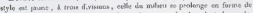

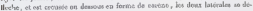
phrea ea bas

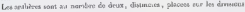

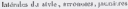

Je o an point vit le firnt le ceste placio

\section{HISTOIRE}

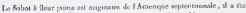

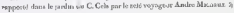

II Lans as prou ber

$$
\text { ODSIRYATHONS. }
$$

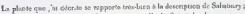

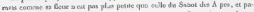

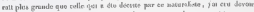

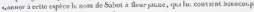

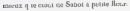

\section{X P L C A T IN DE L \& F L N C L L}

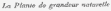

1. Ie lolvo is otgle ru an Jeunas.

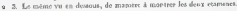




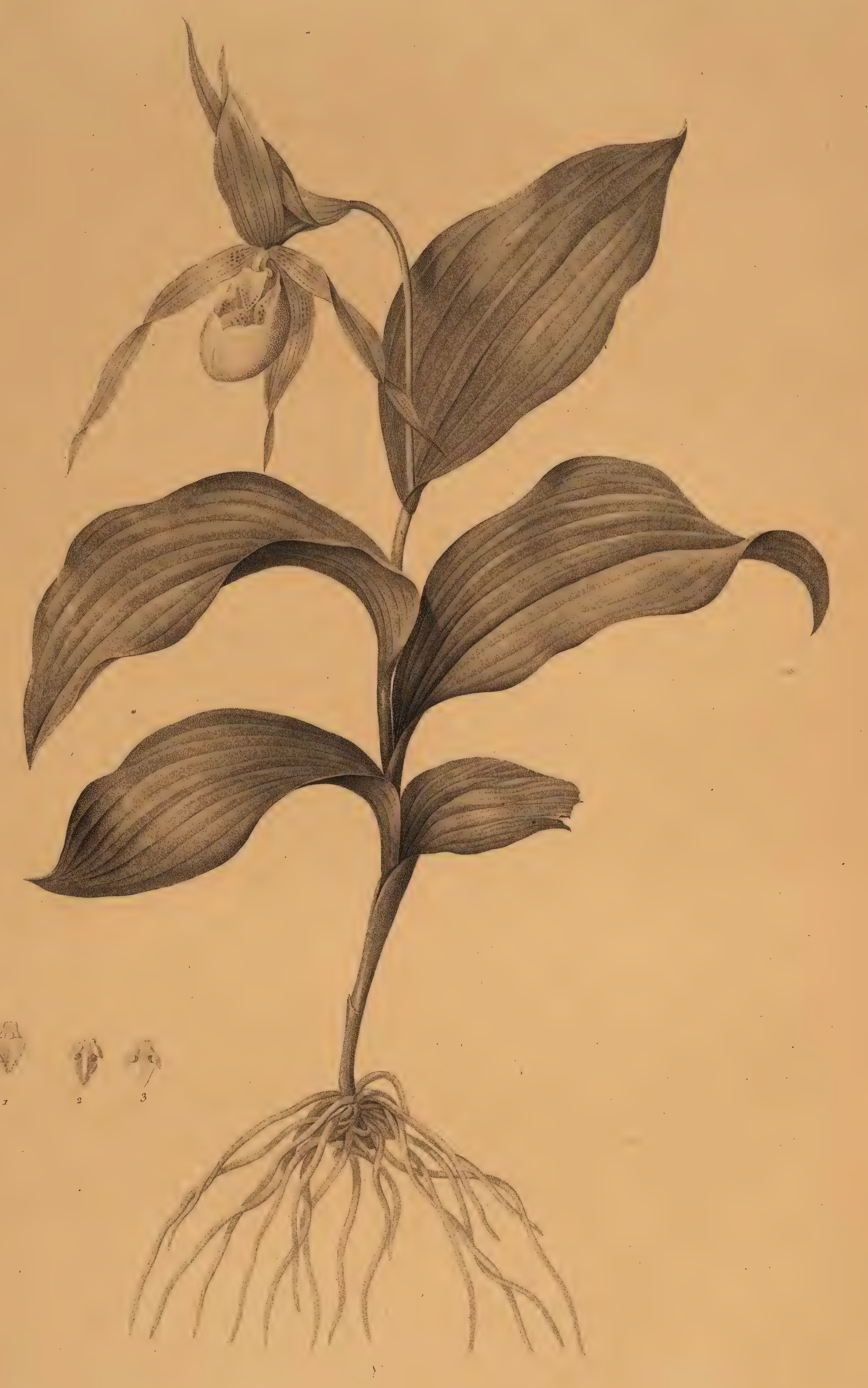

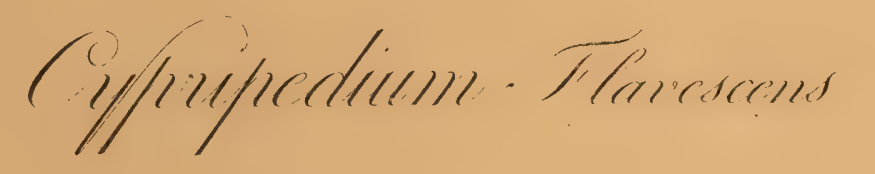

3 Satces is flime pirmene 


$$
\text { 战 }
$$




\title{
ALBUCA MIYOR
}

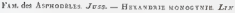

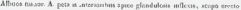

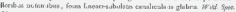
PI s is iso

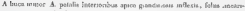

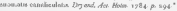

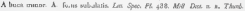
probs 6.5

Alenex, laba vari a Cue ben is a pe

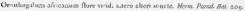
4 abs

\section{ALBUCA JACNATHE.}

\section{J E 8 C $\mathrm{H}+\mathrm{p}$ \% $10 \mathrm{~N}$}

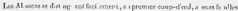

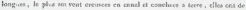

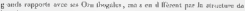

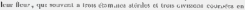
desiros vecs fcur so:n ac

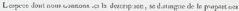

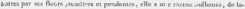

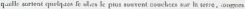

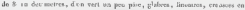

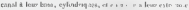

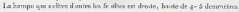

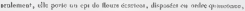

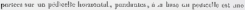

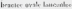

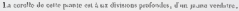

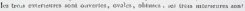

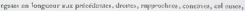

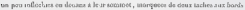
- vecioma 


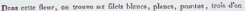

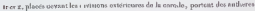

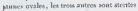

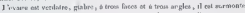

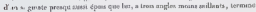

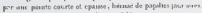

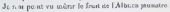

\title{
HI ST T I E.
}

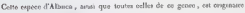

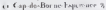

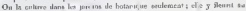

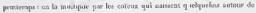
at balaes

\section{EXPLICATION DE LA PLA $\mathrm{X}$ CH}

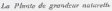

\author{
+ Ie fis ur saniove

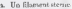 \\ 4 In umena esteracure \\ 4 Eno dirmos wienous \\ I I oe dian en fertik

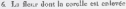 \\ 7 Lovom \\ 8 Lofruic \\ 9. U'ostrains
}




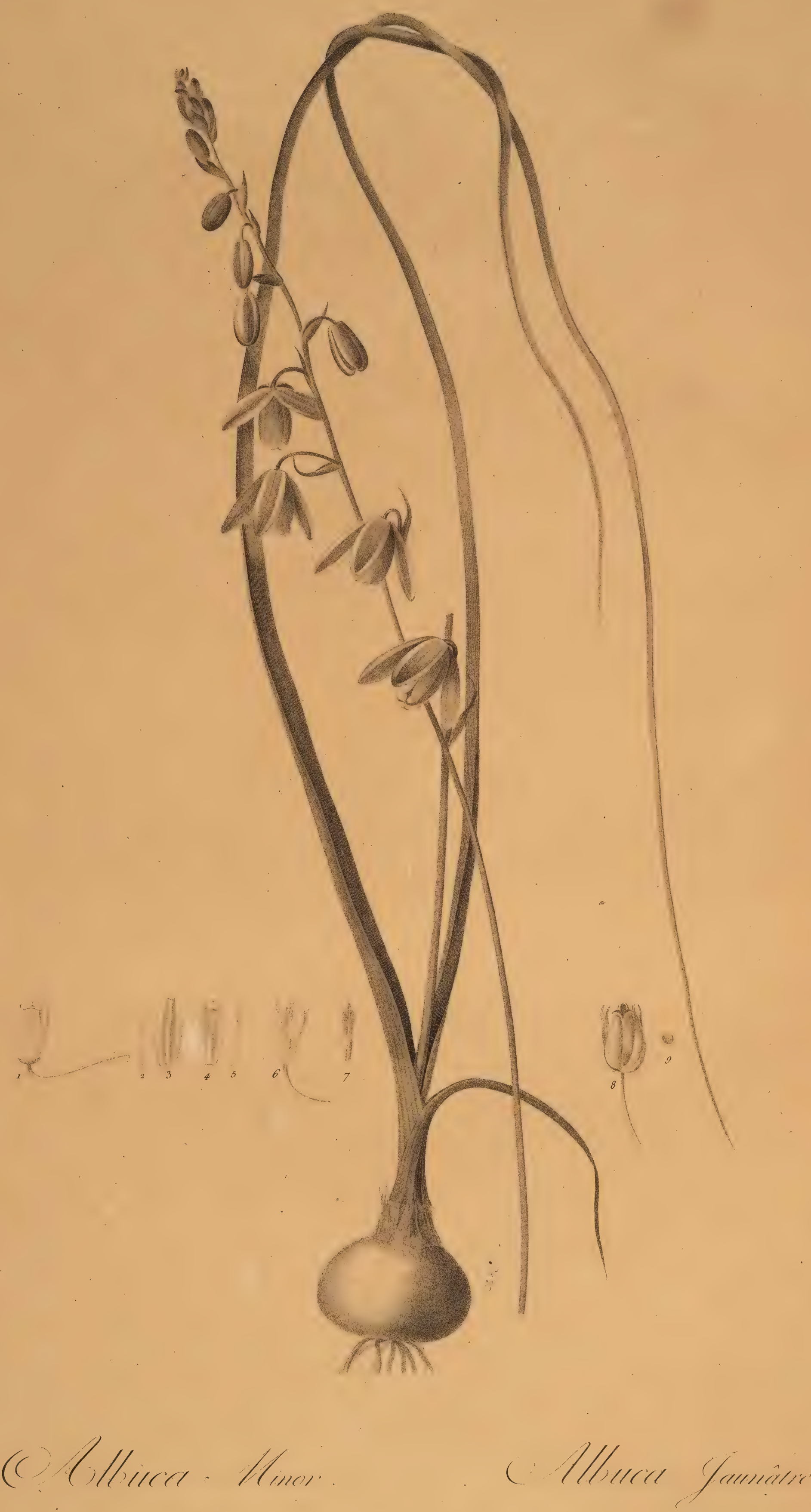




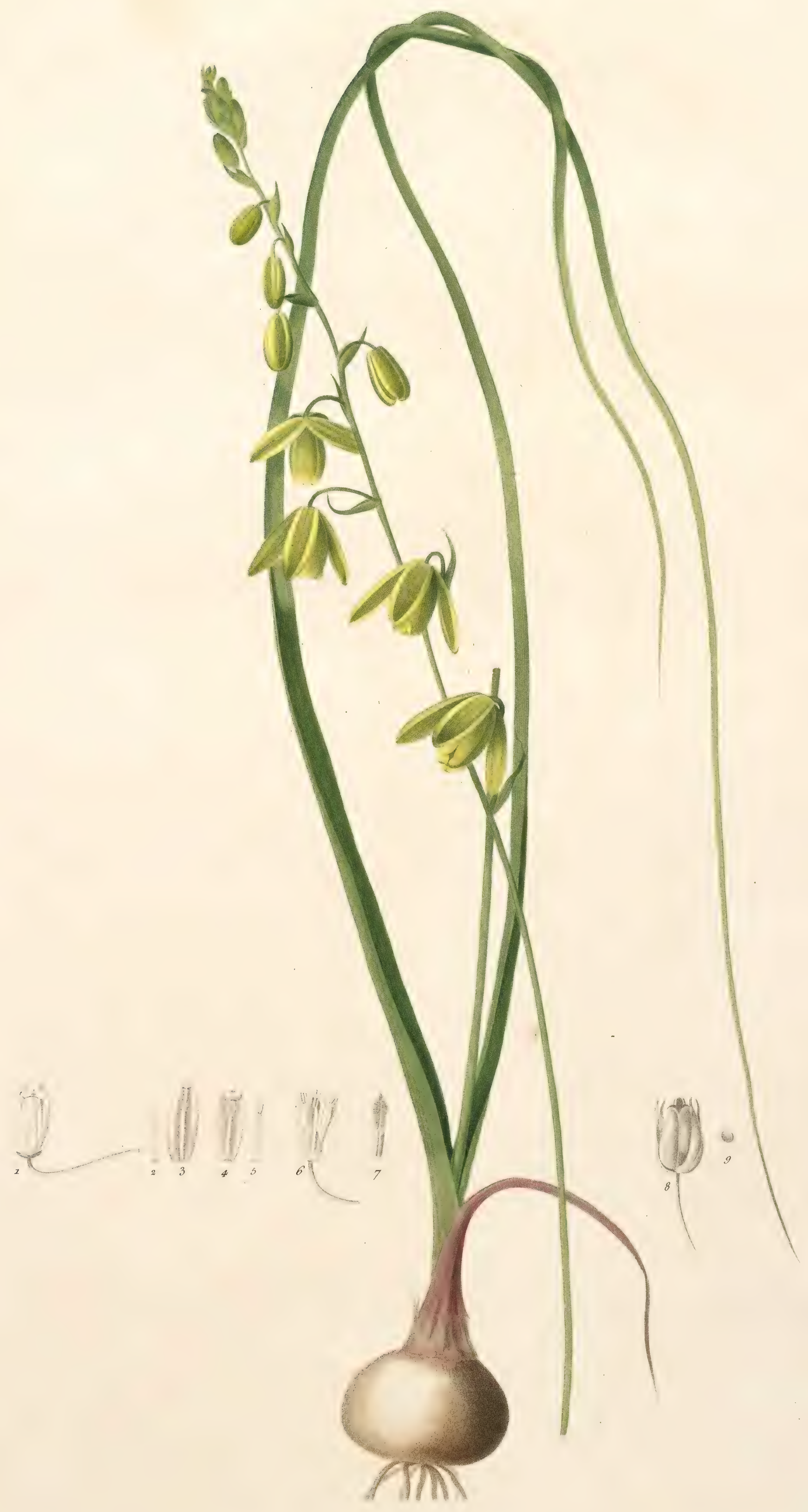

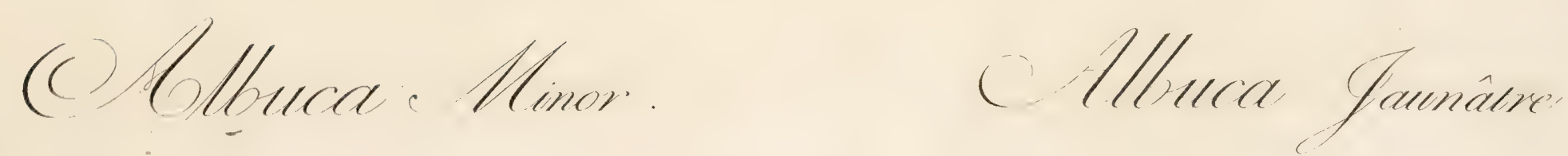




\section{LACIENALIA PALLTDA.}

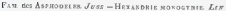

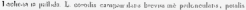

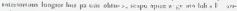

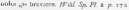

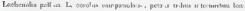

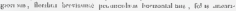

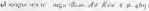

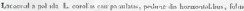

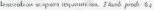

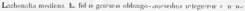

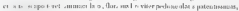

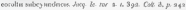

\section{LACHEN $\triangle \mathrm{LE} P \perp L E$.}

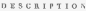

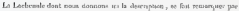

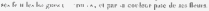

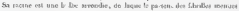

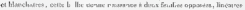

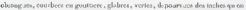

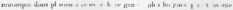

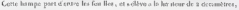

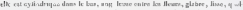

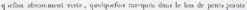

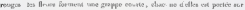

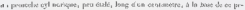

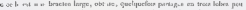

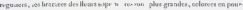

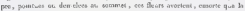

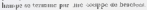

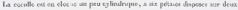

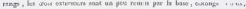

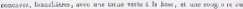




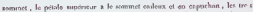

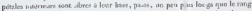

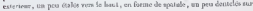

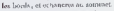

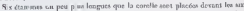

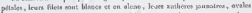

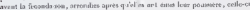

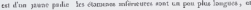

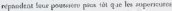

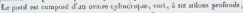

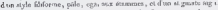

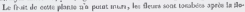
racok

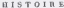

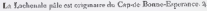

Dum nos jerss. Cle A

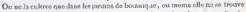

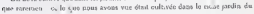
C C... 



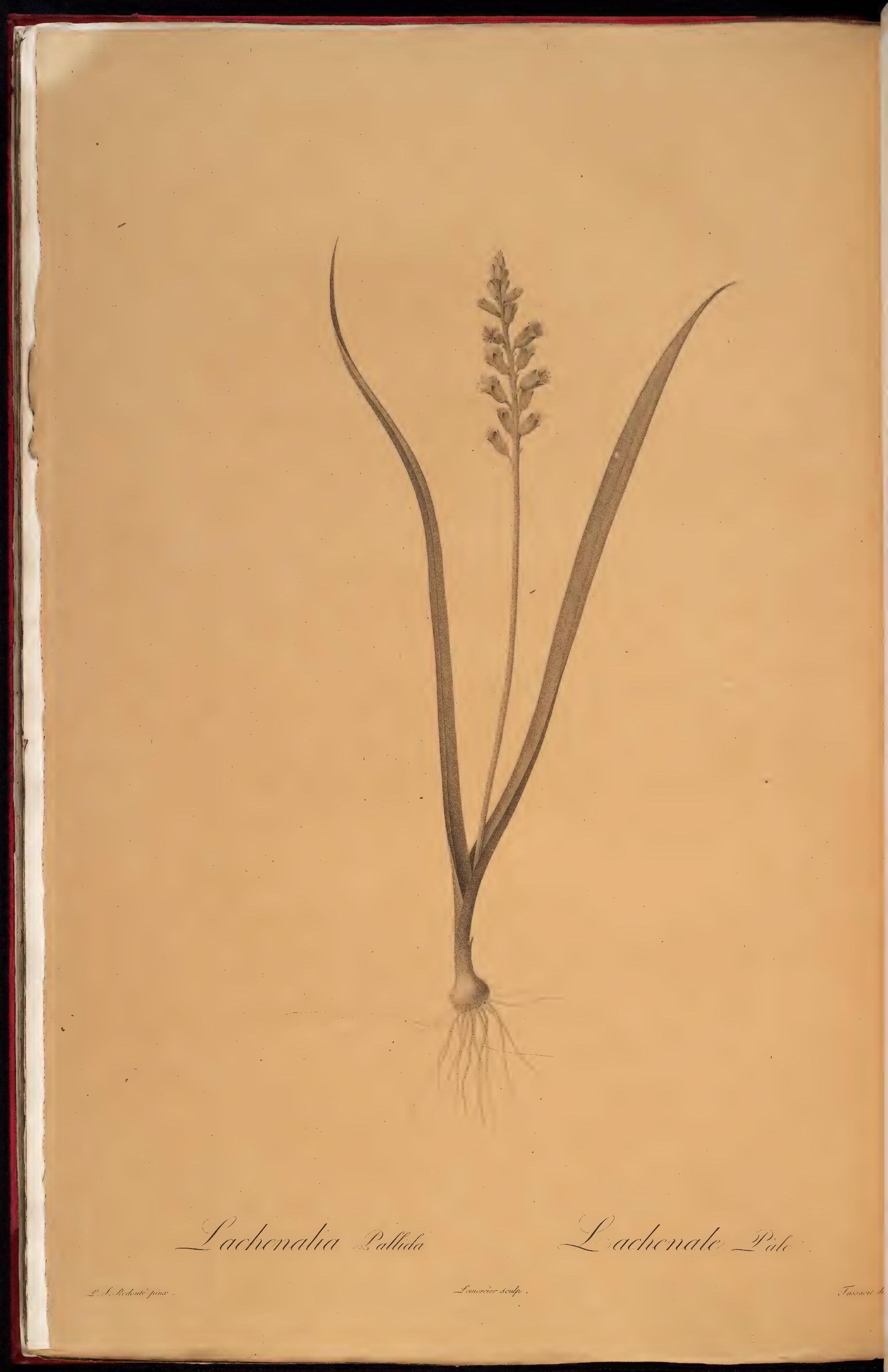




$$
V
$$




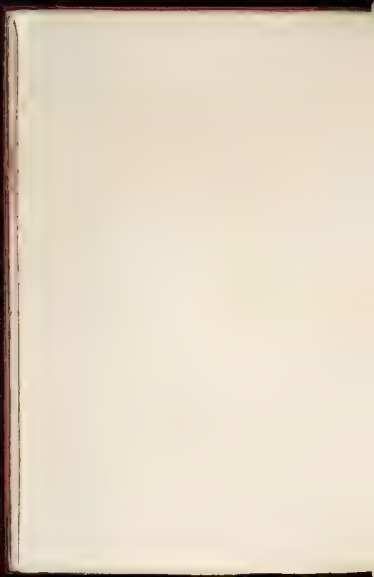




\title{
IRIS FLORENTINA.
}

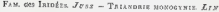

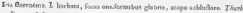
C.ri. x. S.

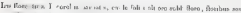

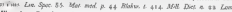

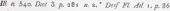

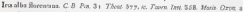

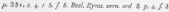

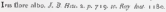

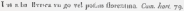

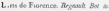

\section{ITIS DE FLOETRCB}

\section{1) $\mathrm{E} S \mathrm{G}$. I T T I O}

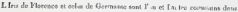

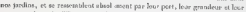

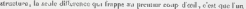

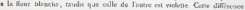

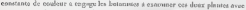

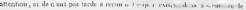

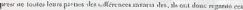

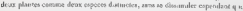

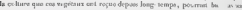

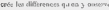

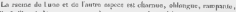

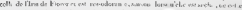

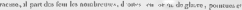

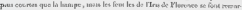

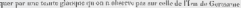

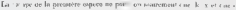

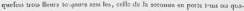

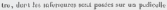




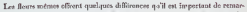

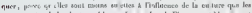

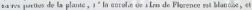

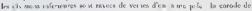

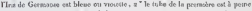

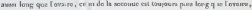

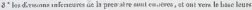

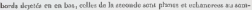

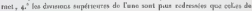

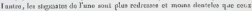
ile inutro

\section{I \& T 0 i I I}

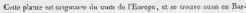
barse : 8

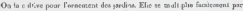
los geuass poels quin pousuat do ia mone 


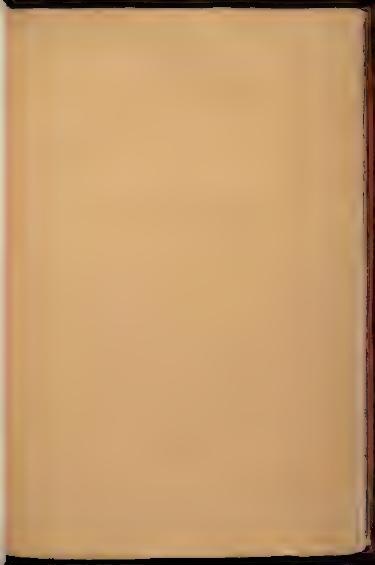




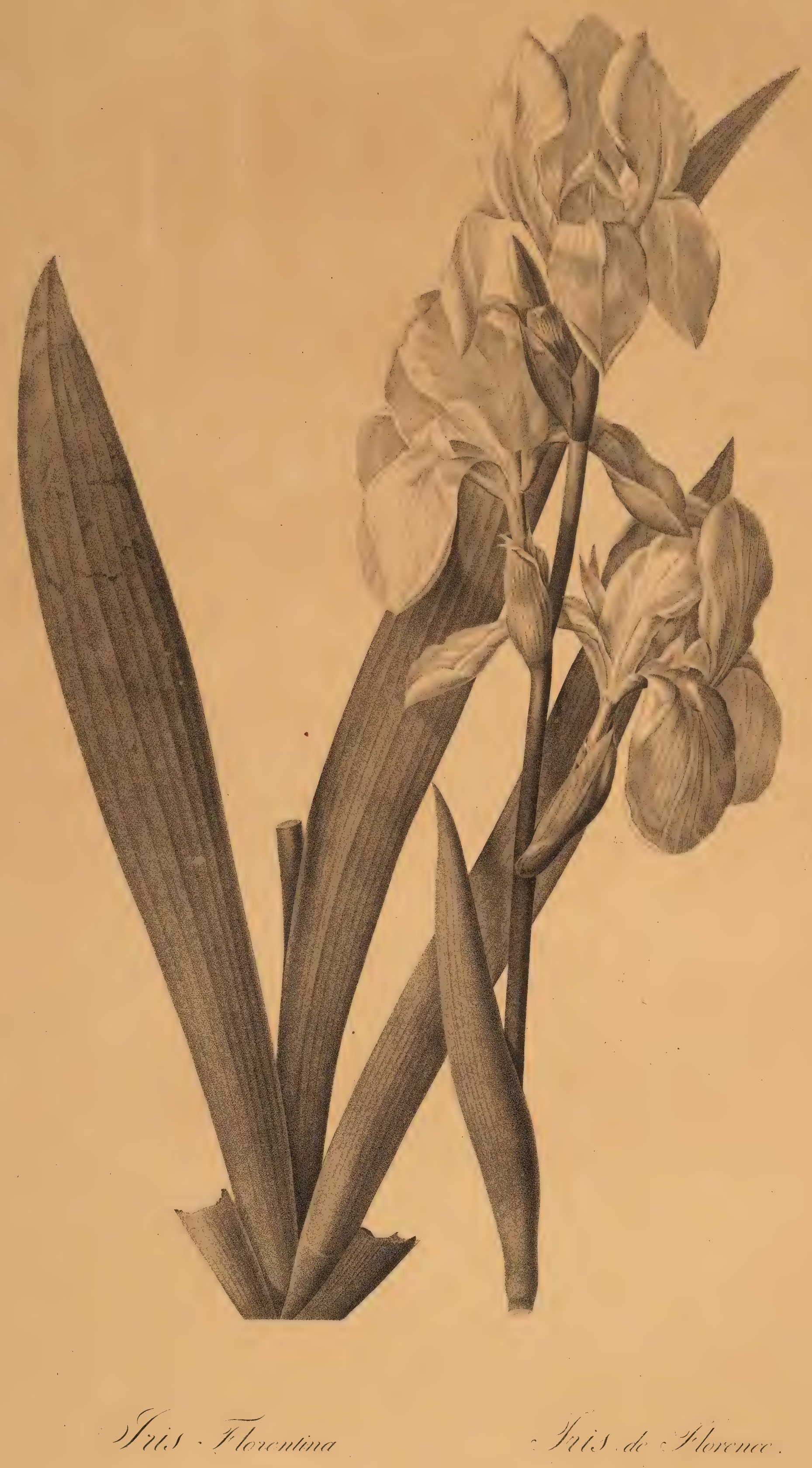




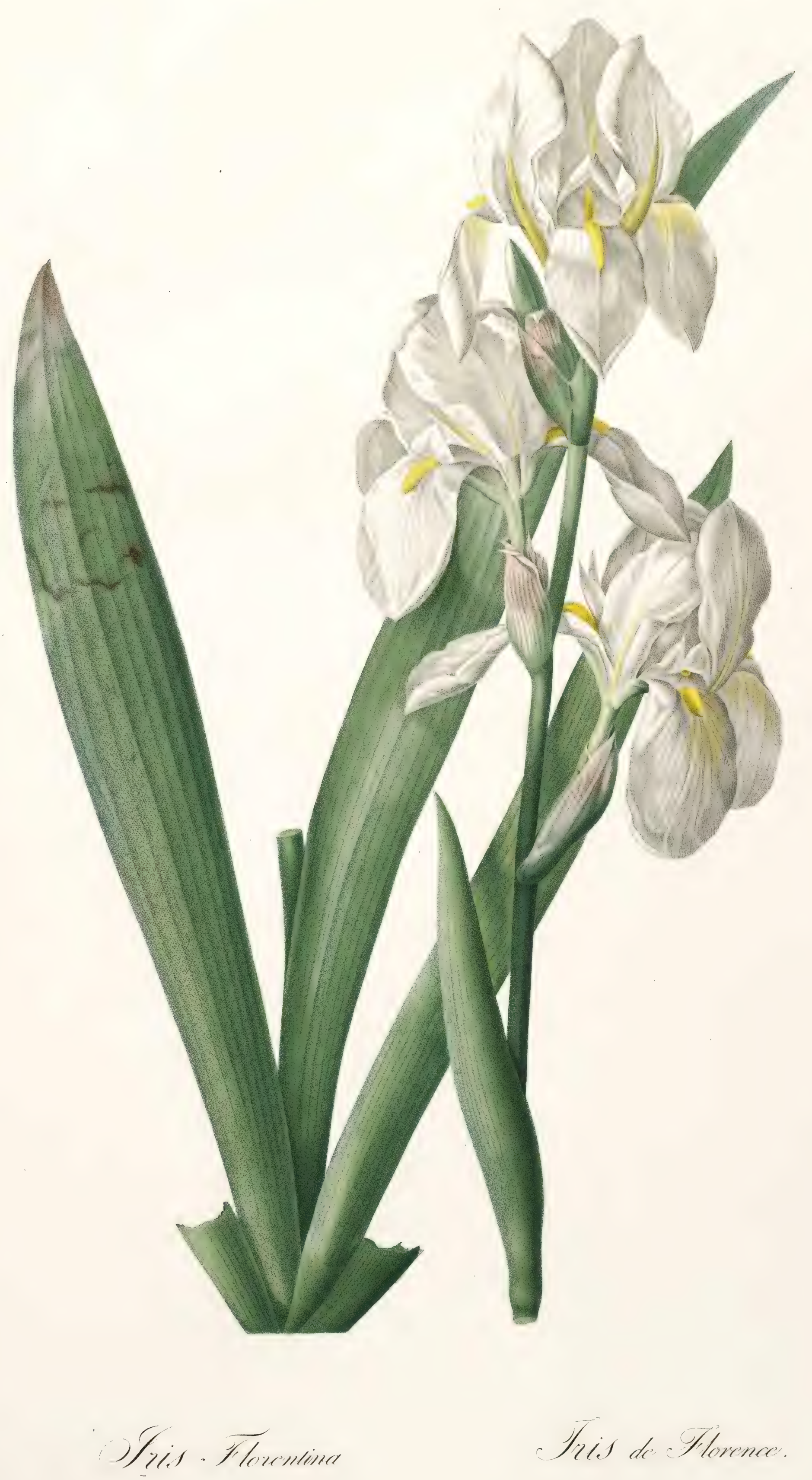




\title{
YARCISSUS BUEBOCODIUM.
}

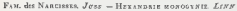

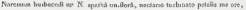

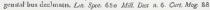

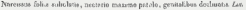

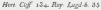

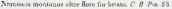

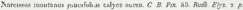
75. 1.5 .9

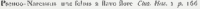

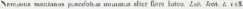
ris

\section{NATCISSE TH LBOCODT.}

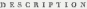

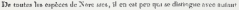

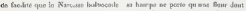

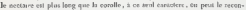

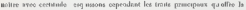

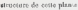

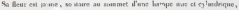

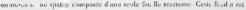

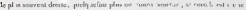

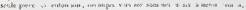

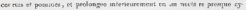

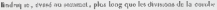

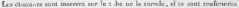

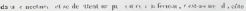

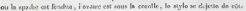

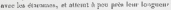

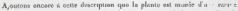

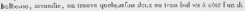

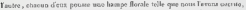

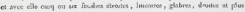




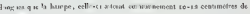
, oag-ate at

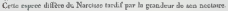

\section{I I I I E}

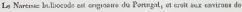
Loboanc 2

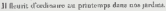

\section{B X L ICA TION DR IA P L A N C I}

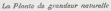

1. Le verule is 0

L Lir post 



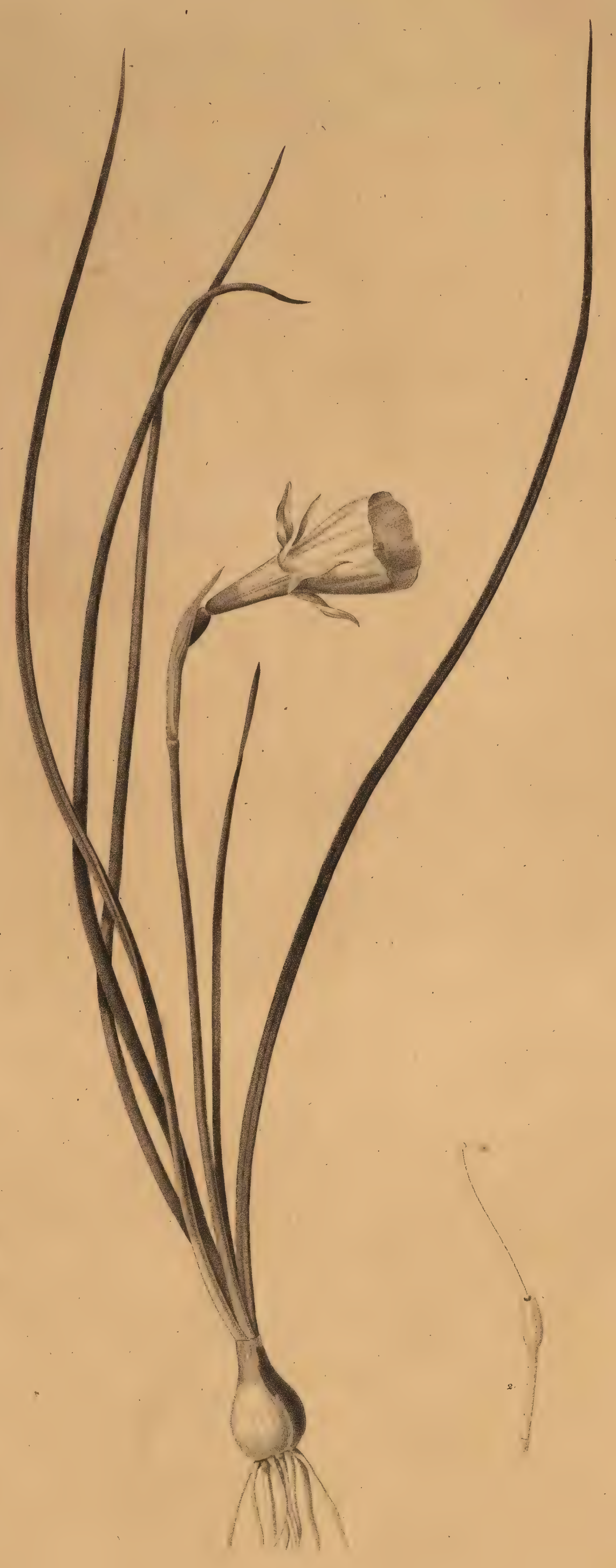

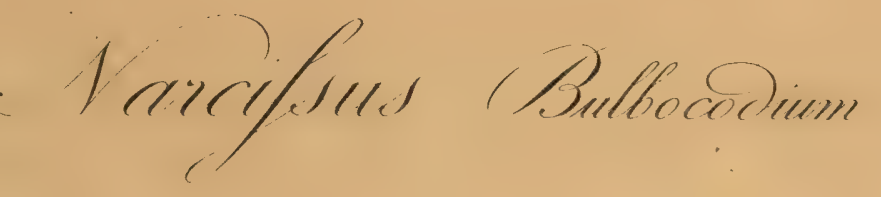

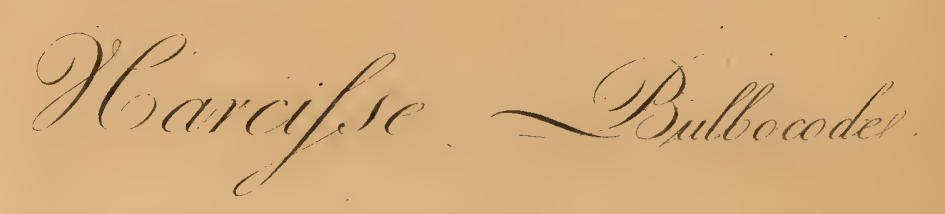





\title{
VEREVDERI BLLBOCODIL
}

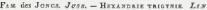

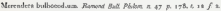

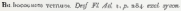

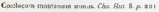

\section{HERENDERE BLLBOCODE}

\section{DESCIIPTION}

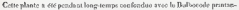

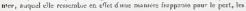

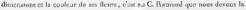

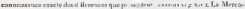

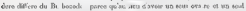

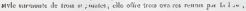

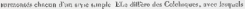

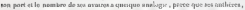

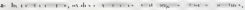

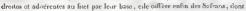

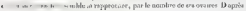

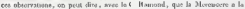

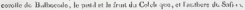

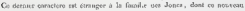

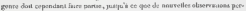

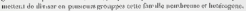

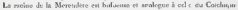

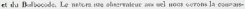

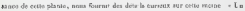

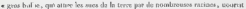

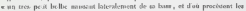

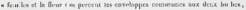

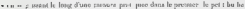

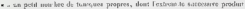

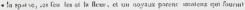

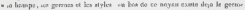

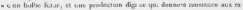

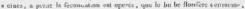

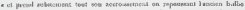

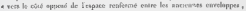




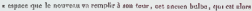

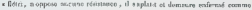

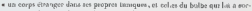

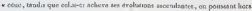

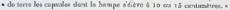

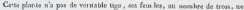

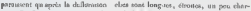

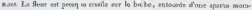

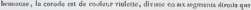

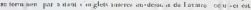

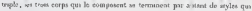

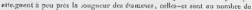

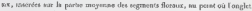

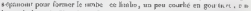

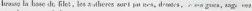

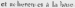

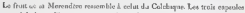

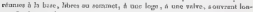

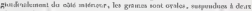

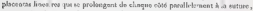
ct «u nitiond of phig remunt Luads isutg.

\section{1 S TO II $\mathrm{T}$}

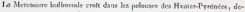

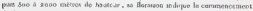

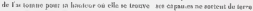

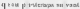

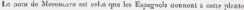
et Alitulevo-annagun:

\section{EXITLIEATION DE LA PI.AKCHE}

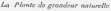

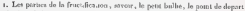

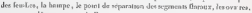

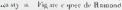

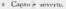

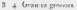

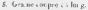

6. Gibane rat peren ravers 



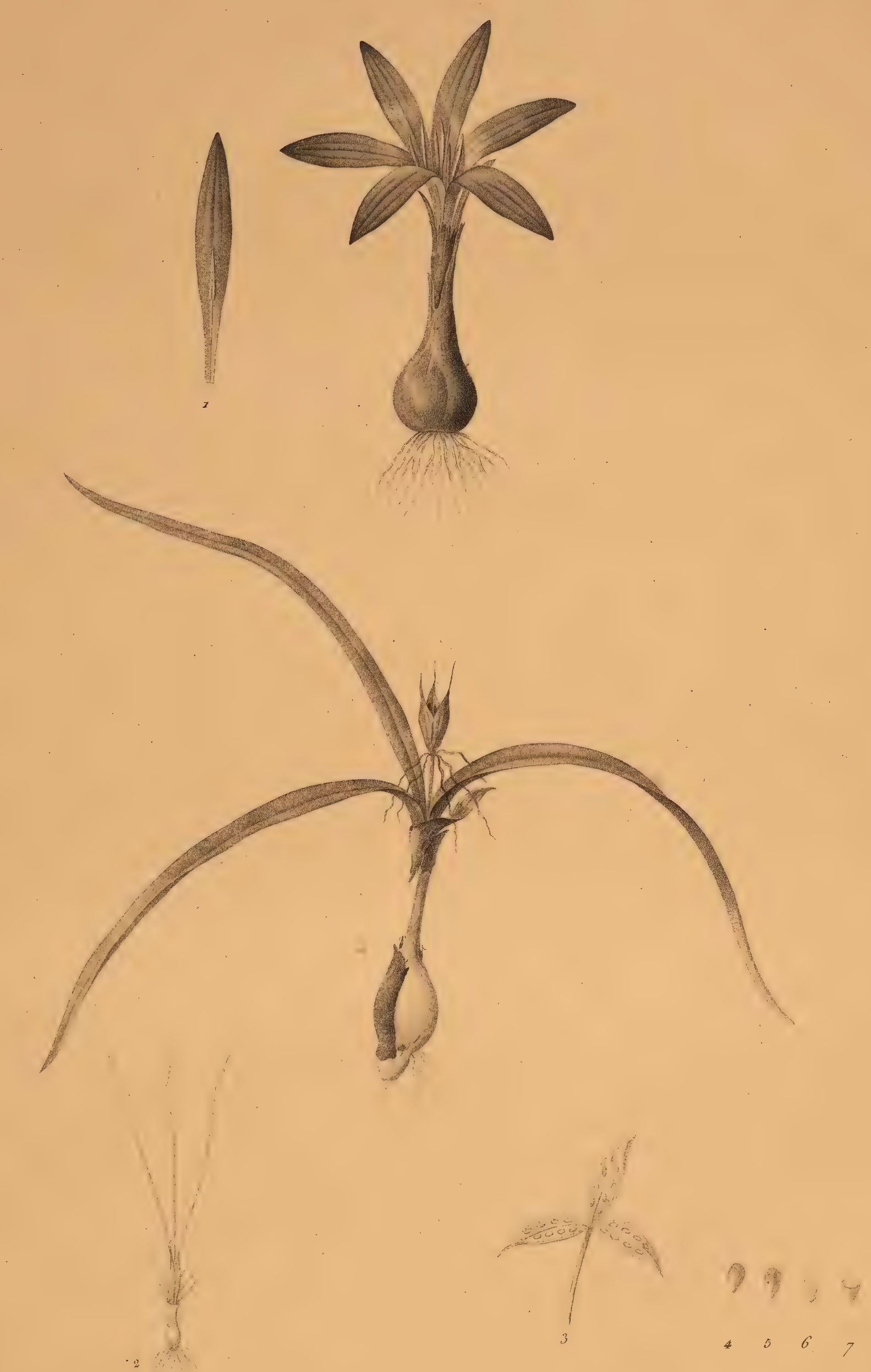

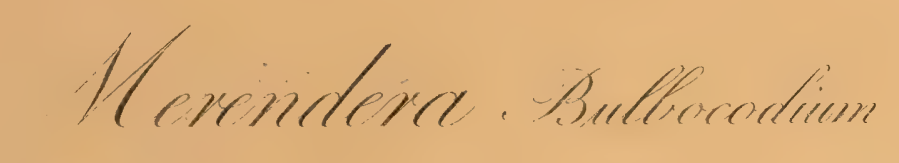

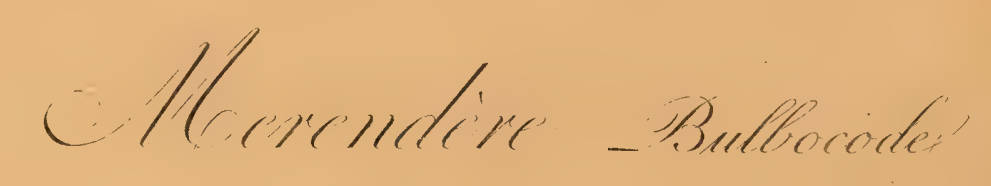




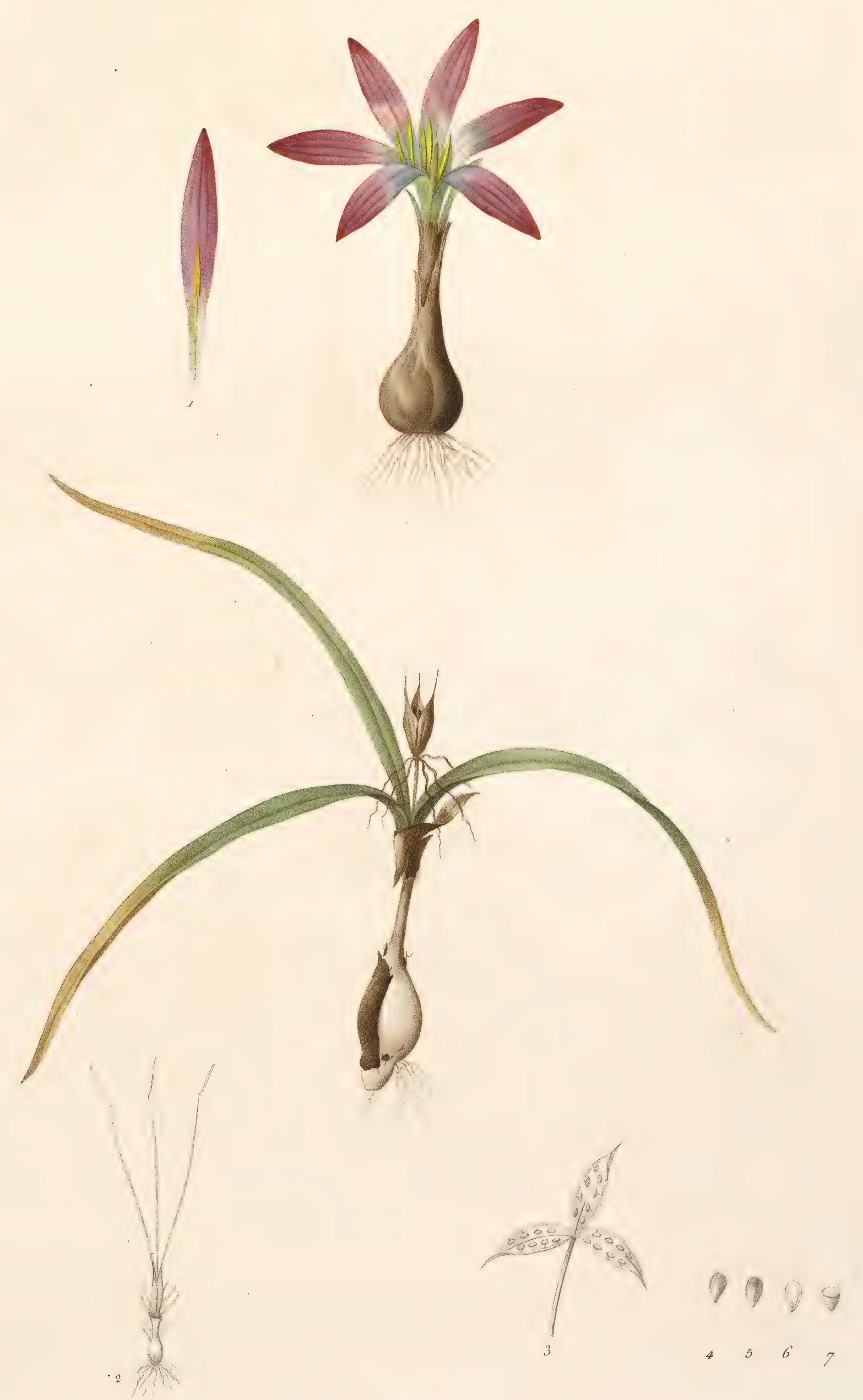

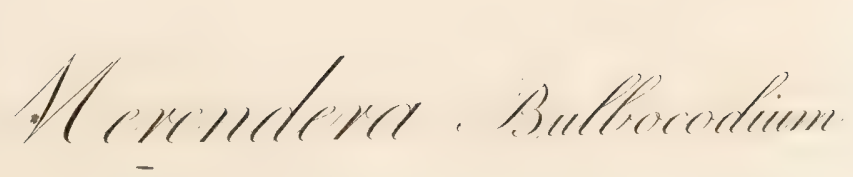

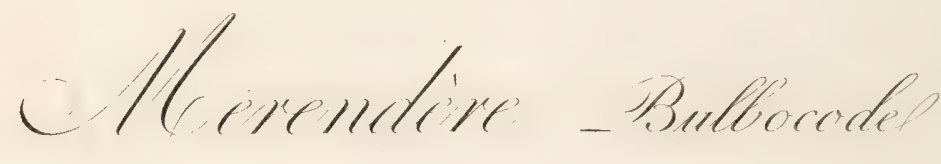




\title{
NETIONIEA SUPERB
}

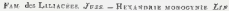

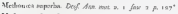

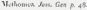

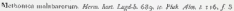

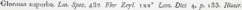

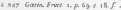

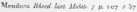

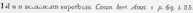

\section{MEIIUNLQLE SUPERBE}

\section{I 8 C I T T T}

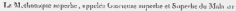

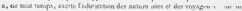

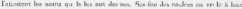

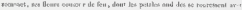

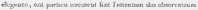

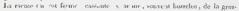

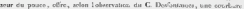

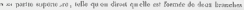

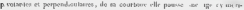

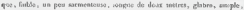

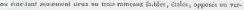
1. 1 .

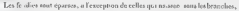

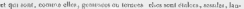

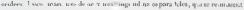

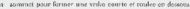

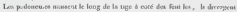

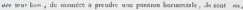

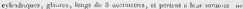
Dum woveire at paechos

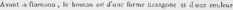

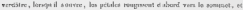

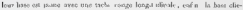

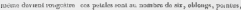




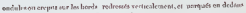

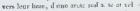

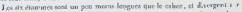

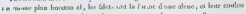

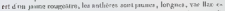

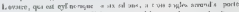

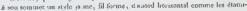

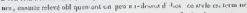

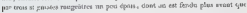
Lan Wate a.iline

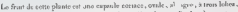

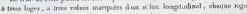

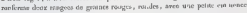
pers ite fun ald

\section{1. $1.50: \pi 1$}

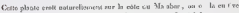

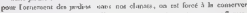

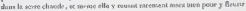

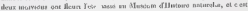

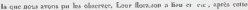

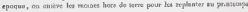
sirvan

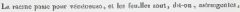

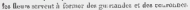

\section{DHSER NAYSONS}

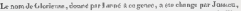

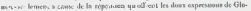

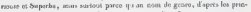

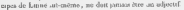

\section{X P I IATION DELA PLANCH B}

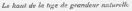

1 Uoc expate en ior

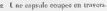

D. I no gruvos 



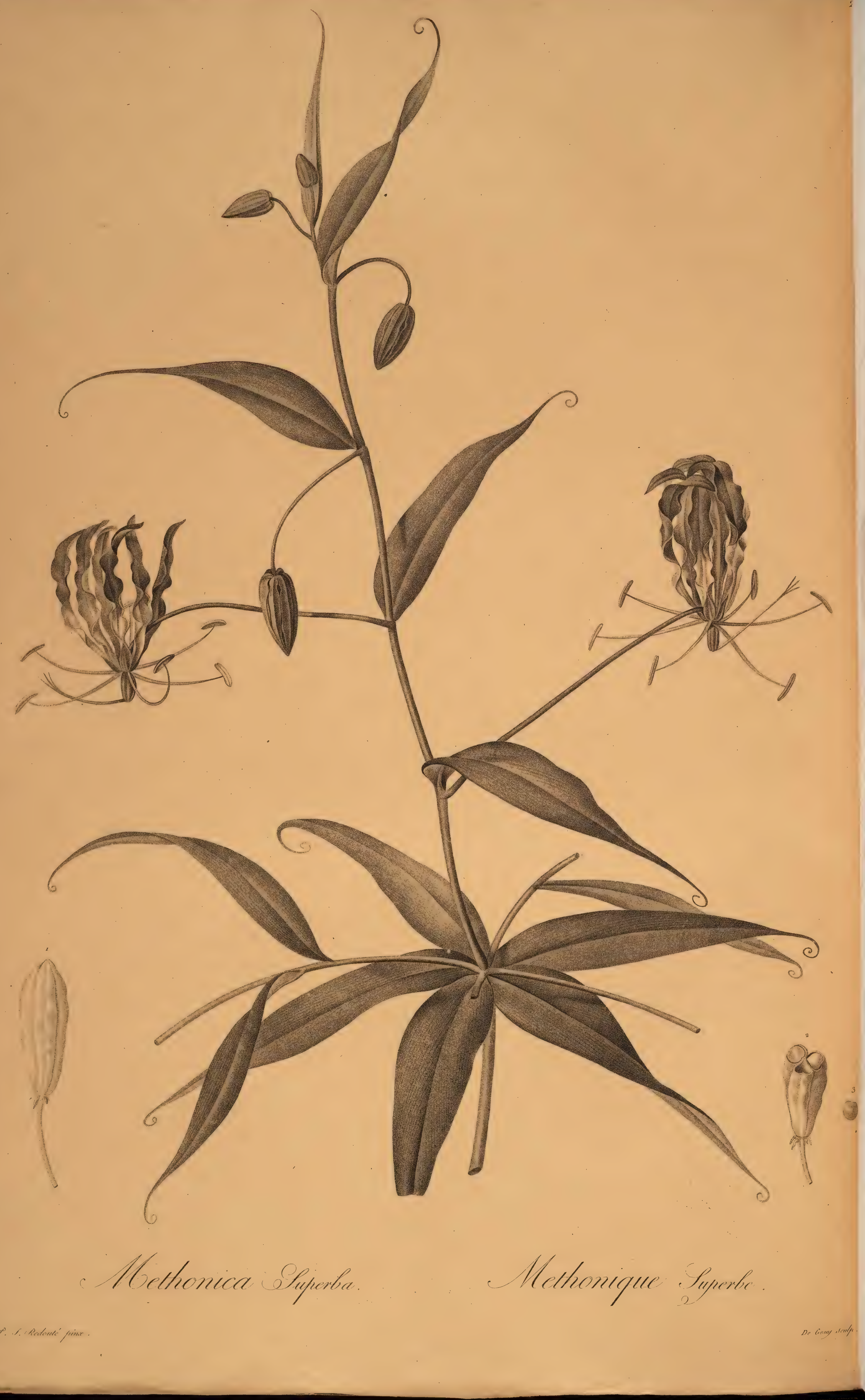




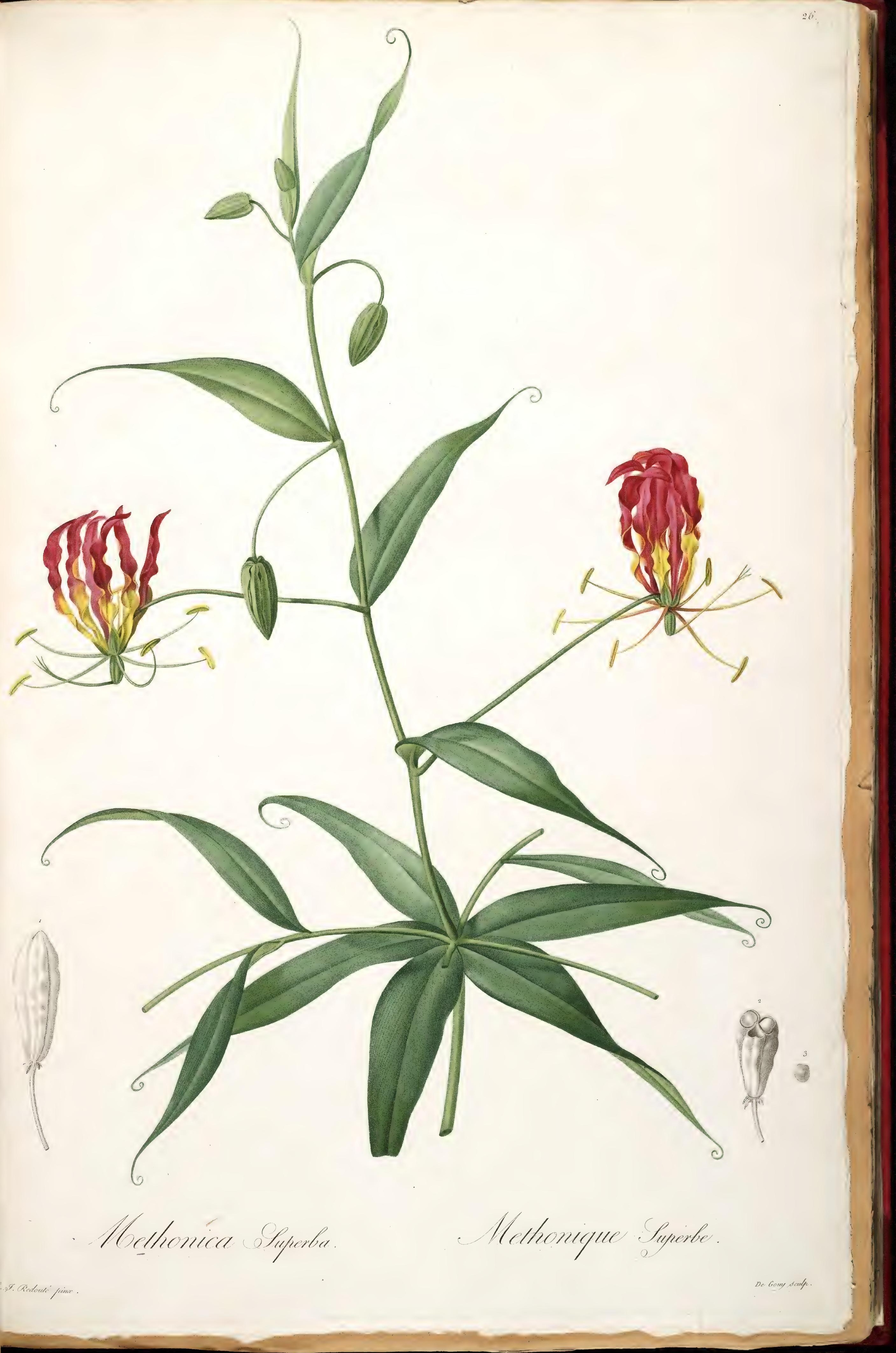




\title{
CRINUA ERUBESCENS.
}

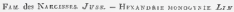

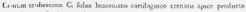

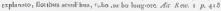

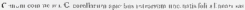

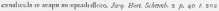

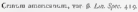

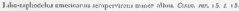

\section{CRTYUA ROTGFATRF.}

\section{J)}

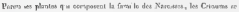

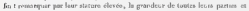

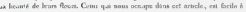

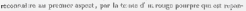

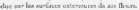

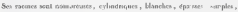

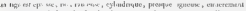

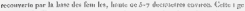

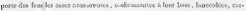

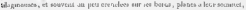

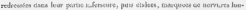
gat al alen.

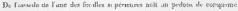

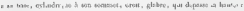

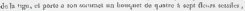

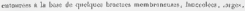

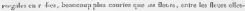

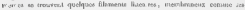
horar etes

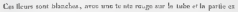

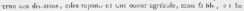

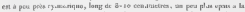

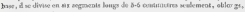
pontus, wowisia 


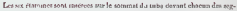

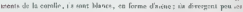

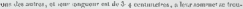

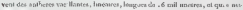
KU I H V pocowne wea is o is a e

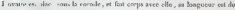

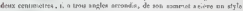

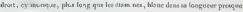

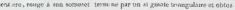

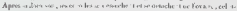

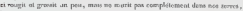

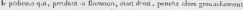

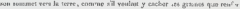

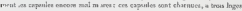
E. arsil vo vos

\section{I $\mathrm{T}$ \% I A F}

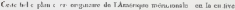

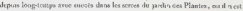

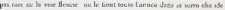

ONSENPA FIOAK

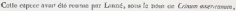

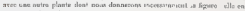

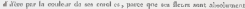

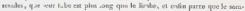

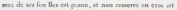





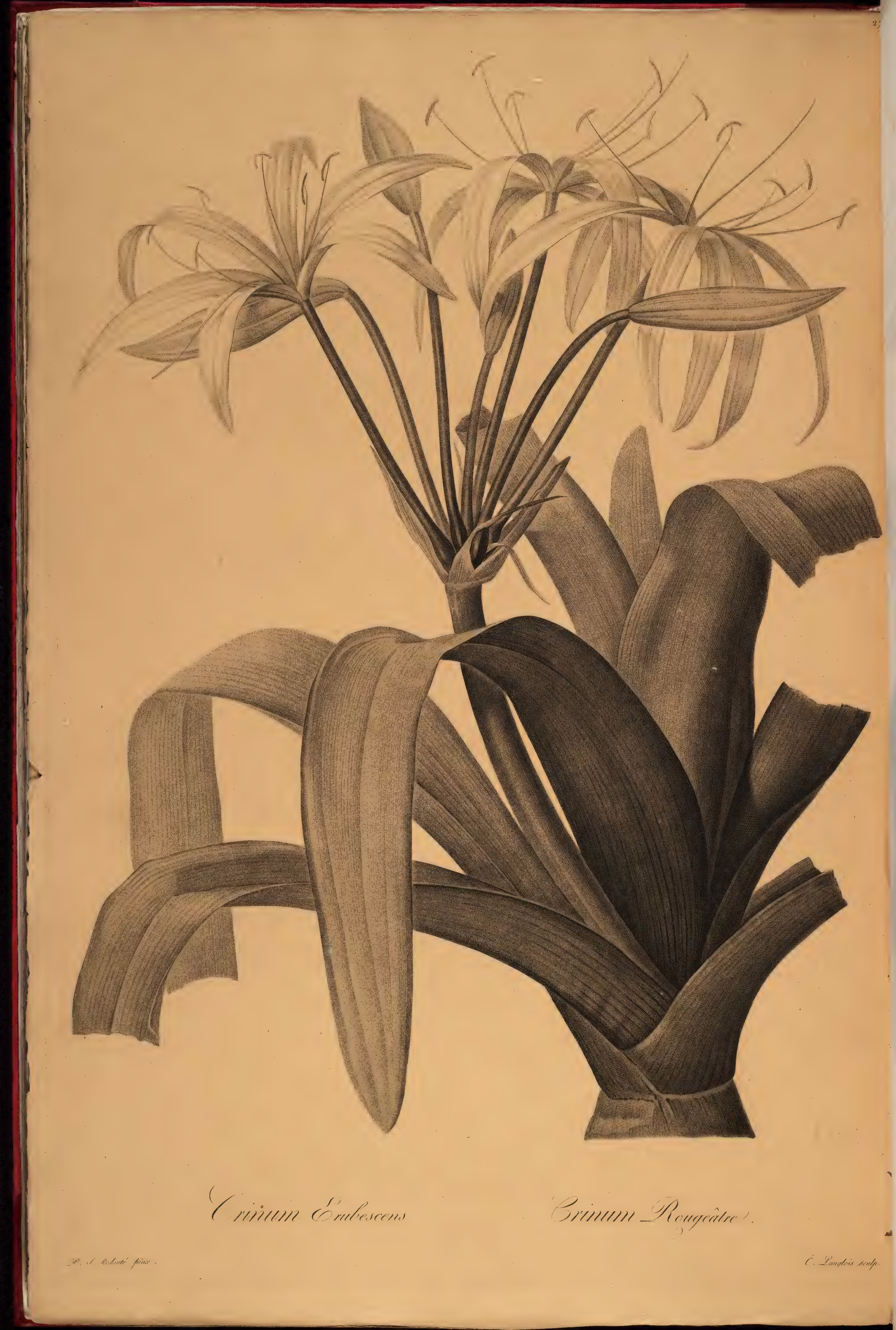




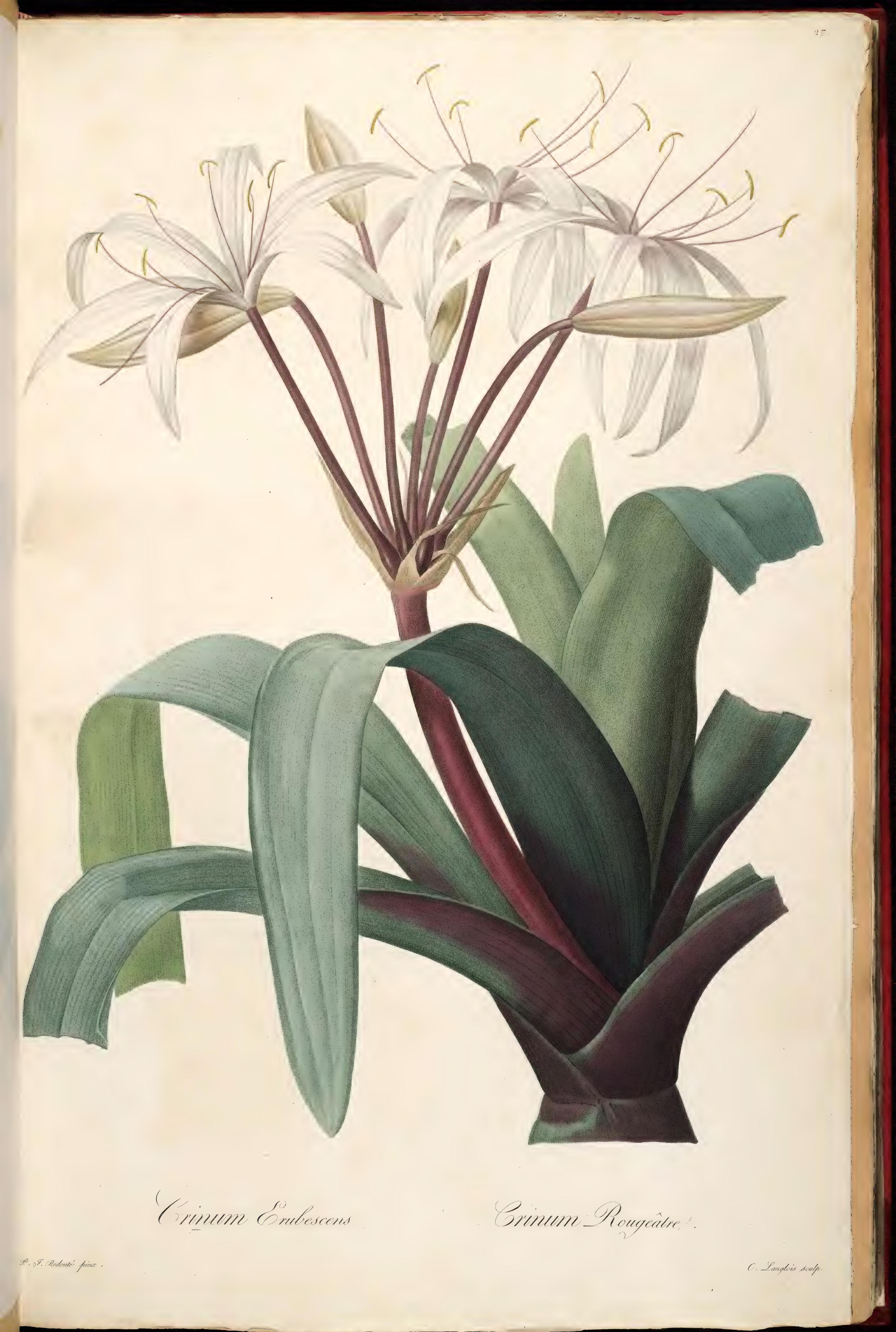




\section{FERRARIA UNDULATA.}

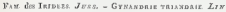

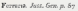

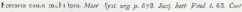

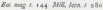

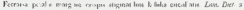
fisti.

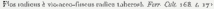

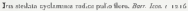

\section{FERARIR ONDULBE.}

\section{DESTIITION.}

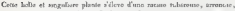

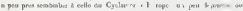

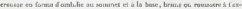

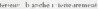

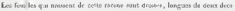

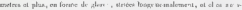

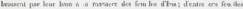

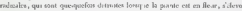

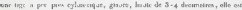

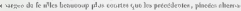

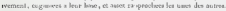

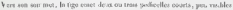

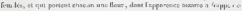

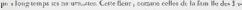

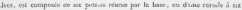

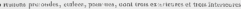

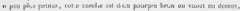

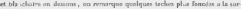

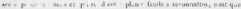

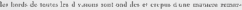
qualite

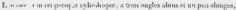

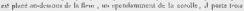




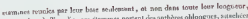

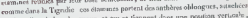

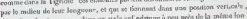

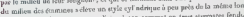

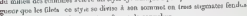

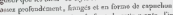

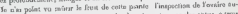

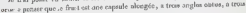

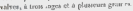

\section{5 \&IR}

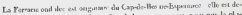

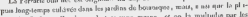

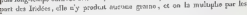
richesen

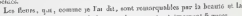

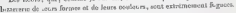

\section{F.XPLICATION DE LA PLANCHL}

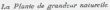

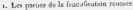

¿ Le puar. 



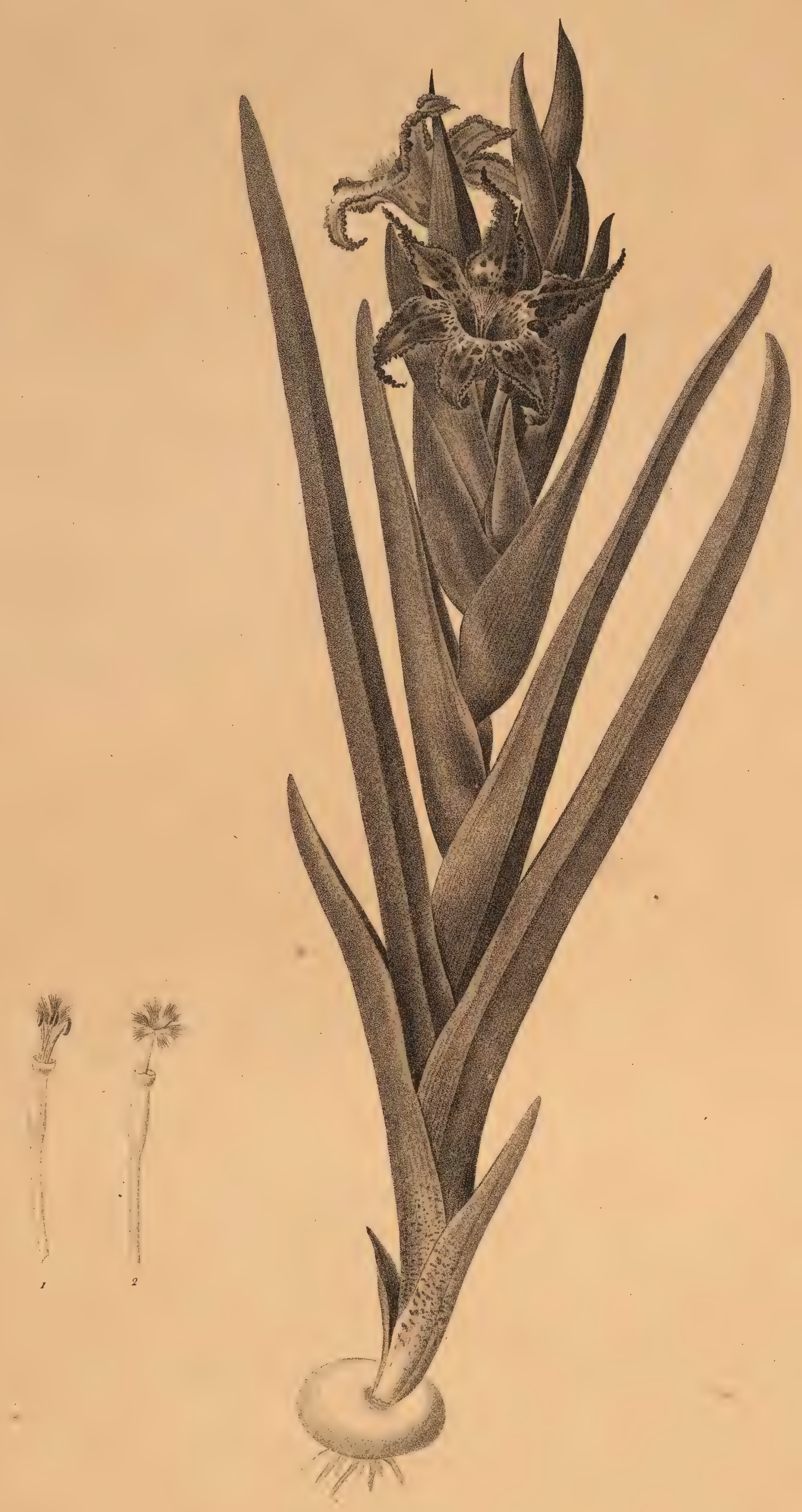

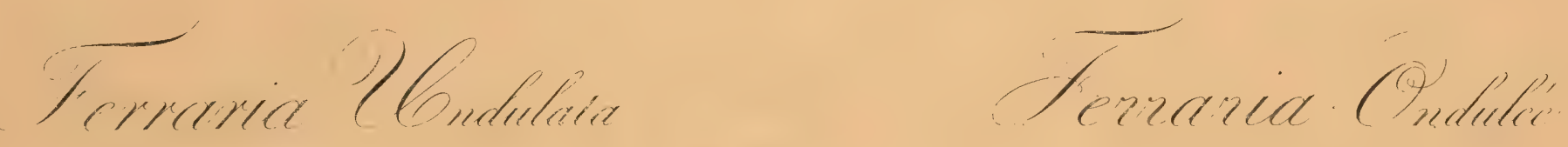




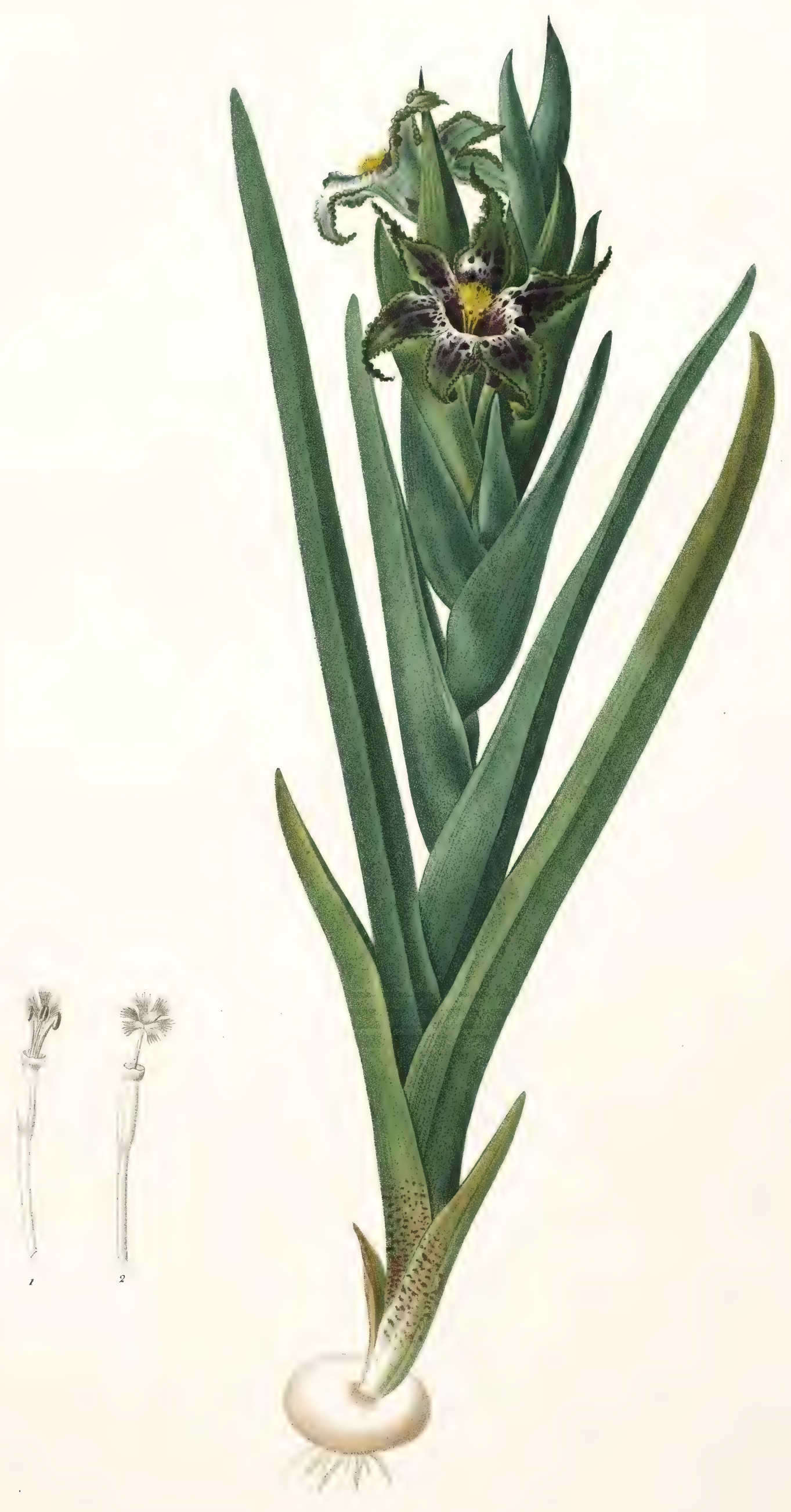

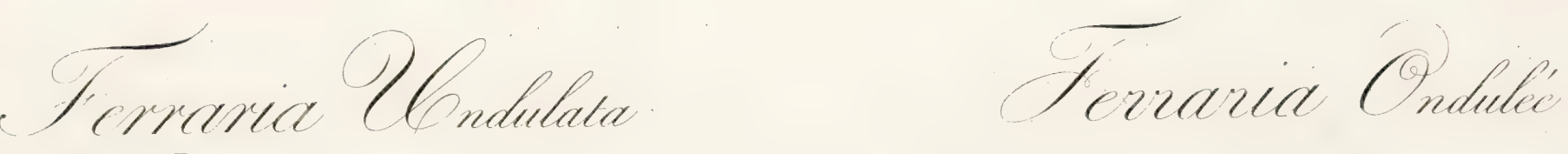





\title{
IRIS SISYRIYCHIUAL.
}

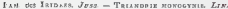

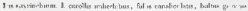

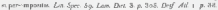

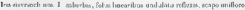

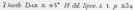

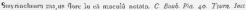

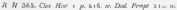

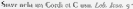

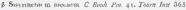

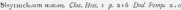

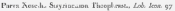

\section{IRIS DOLBLF-ELLB B}

\section{DESCRIPTION}

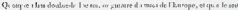

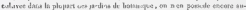

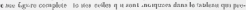

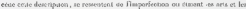

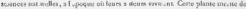

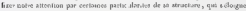

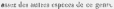

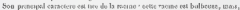

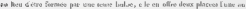

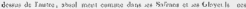

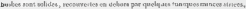

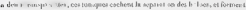

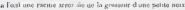

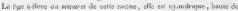

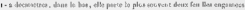

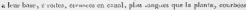

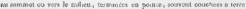
A ra ia de loan fohway

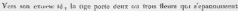

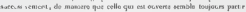




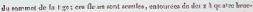

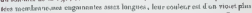

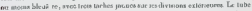

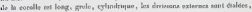

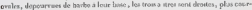
Le pue lys priculesces

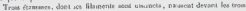

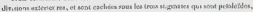

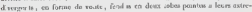

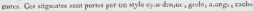

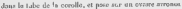

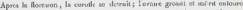

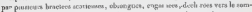

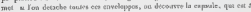

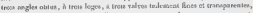

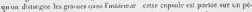

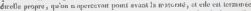

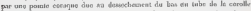

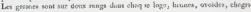

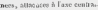

\section{I T T I T}

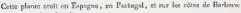

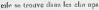

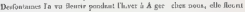
gonlemod at pha-exp

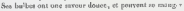

\section{X LICATIQN DE LA PLAXCH}

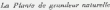

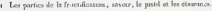

× L. Cronit moavers des spades.

3. La rasules

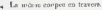

5. 12 melrrows imaits 


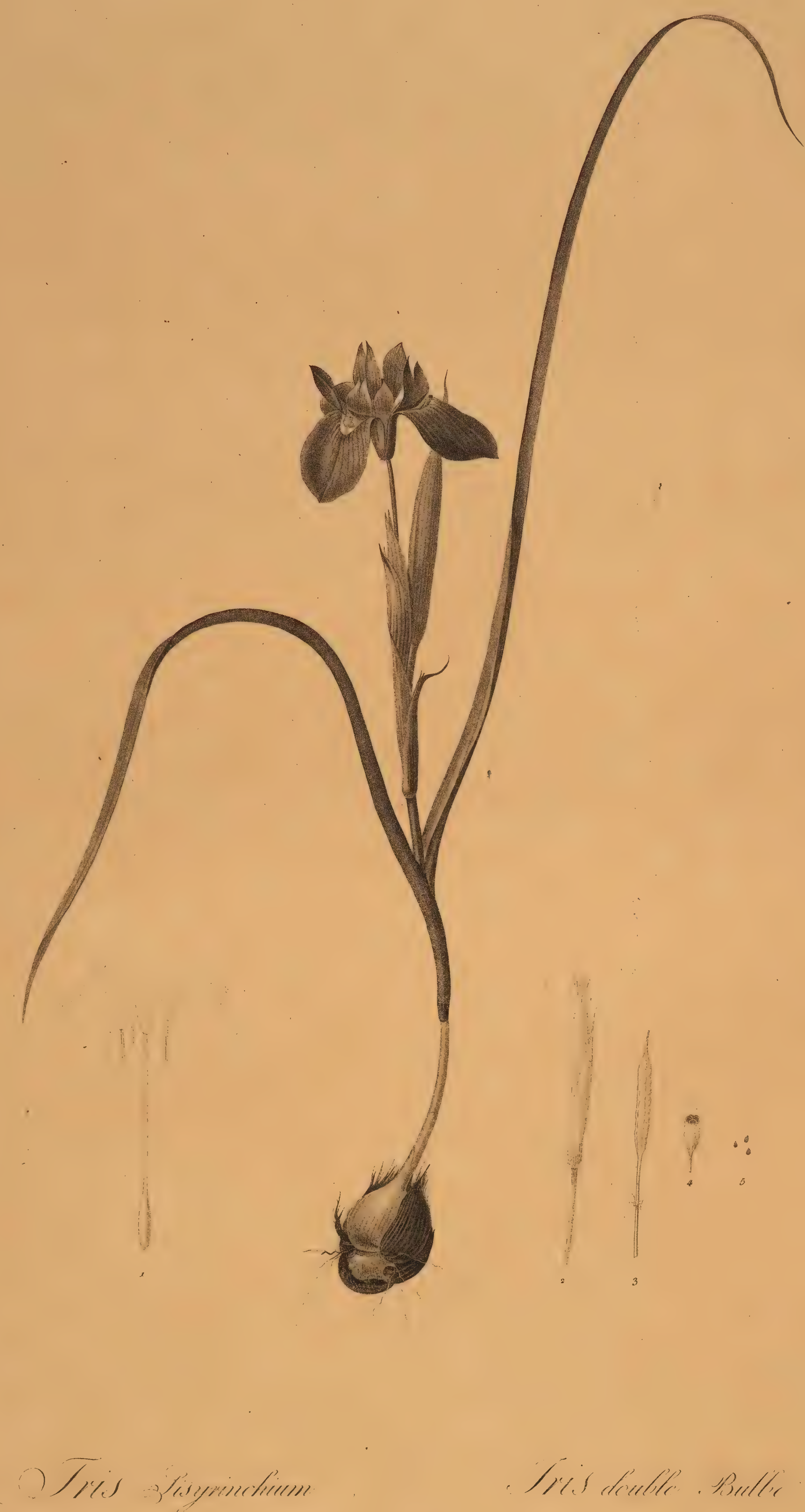






\title{
IXIA FILIFORMIS.
}

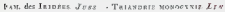

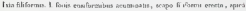

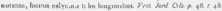

\section{IXIA F ILIFOH AI F}

\section{DESEIITIO}

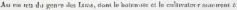

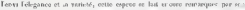

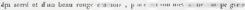
et driter

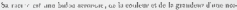

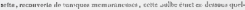

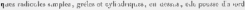

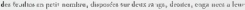

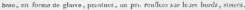

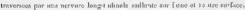

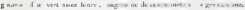
sulinats.

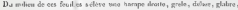

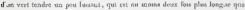

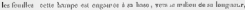

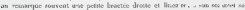

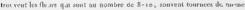

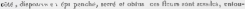

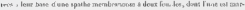

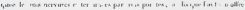

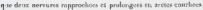

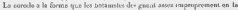

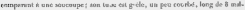

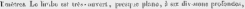

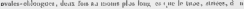

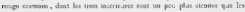
in ares

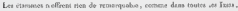

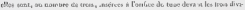




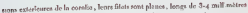

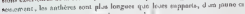
pin, diviles, 11 .

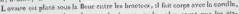

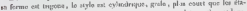

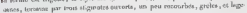

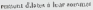

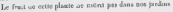

\section{[ 1 \& T 0 : $\mathrm{n}$ \%}

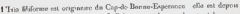

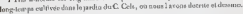

\section{EXPCACATION DE LA HLANCIE}

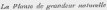

2. Yithos lo la spailia

5. Flour yue pas depsot at uavere paur monirer Tabstiga at ha forave fro 4 Nas int

4 Pisul. 


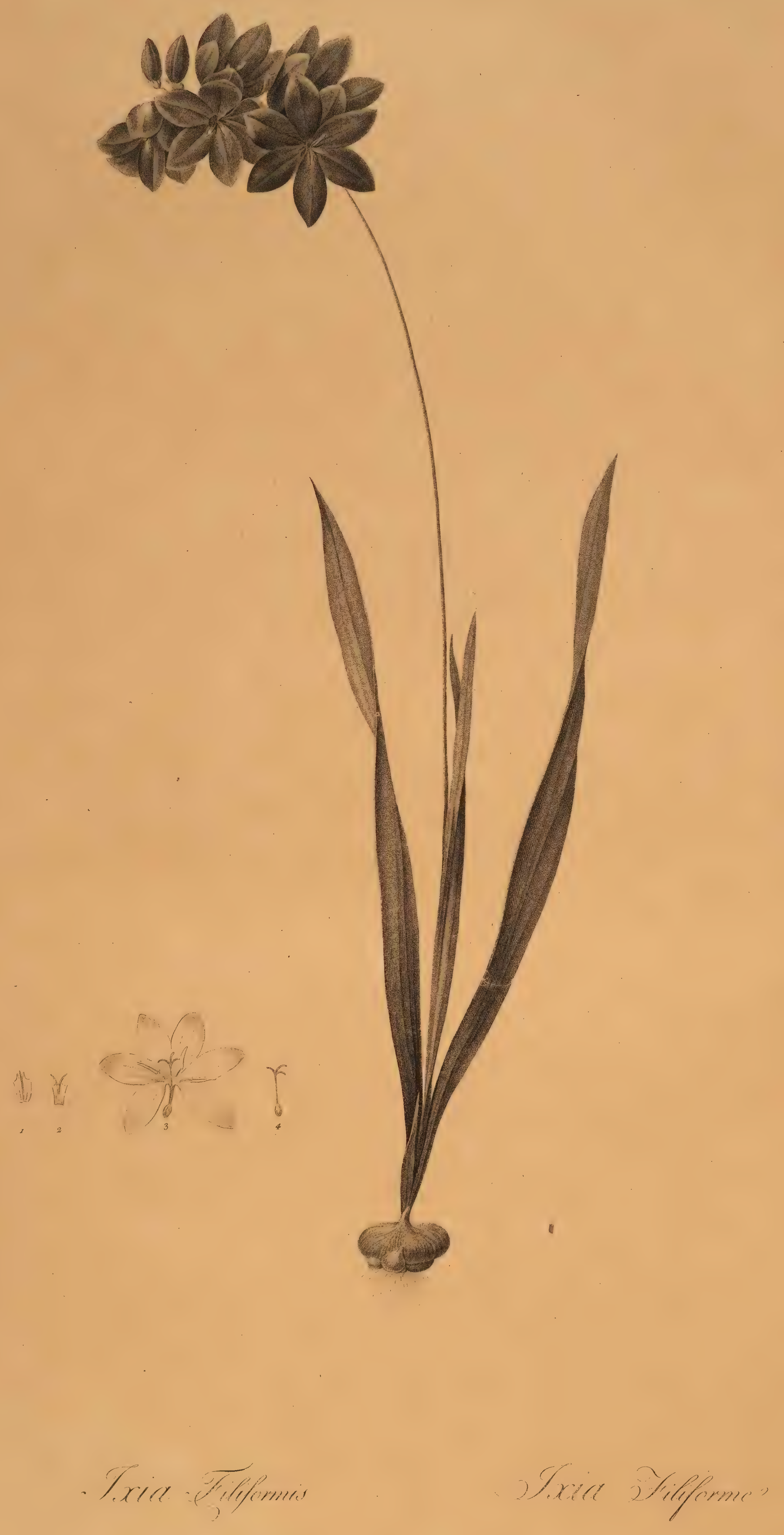




$$
\bar{V}
$$





\section{AMARYLIIS ATAMASCO.}

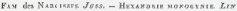

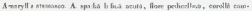

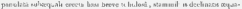

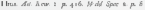

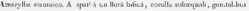
ith 1 a is. L Her. Bire is

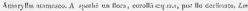

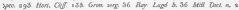

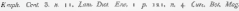
7. 935

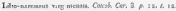

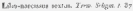

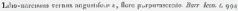

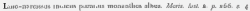
1.24. 4 . Tonro Jese 386

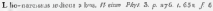

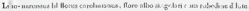

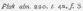

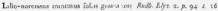

\section{A HAESLLIS DE VIIIGINIE.}

\section{USCNTTETON}

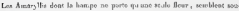

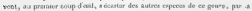

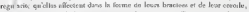

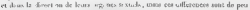

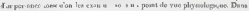

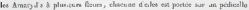

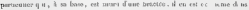

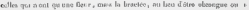

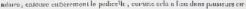

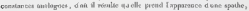

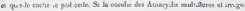




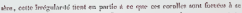

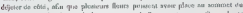

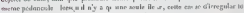

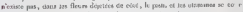

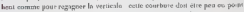

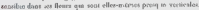

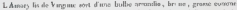

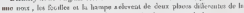

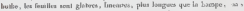

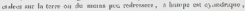

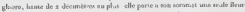

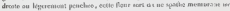

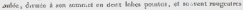

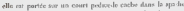

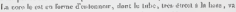

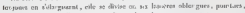

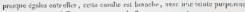

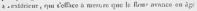

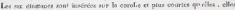

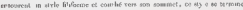

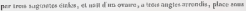
Lit otol

\section{日1 B T O T H}

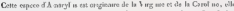

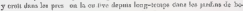

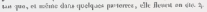





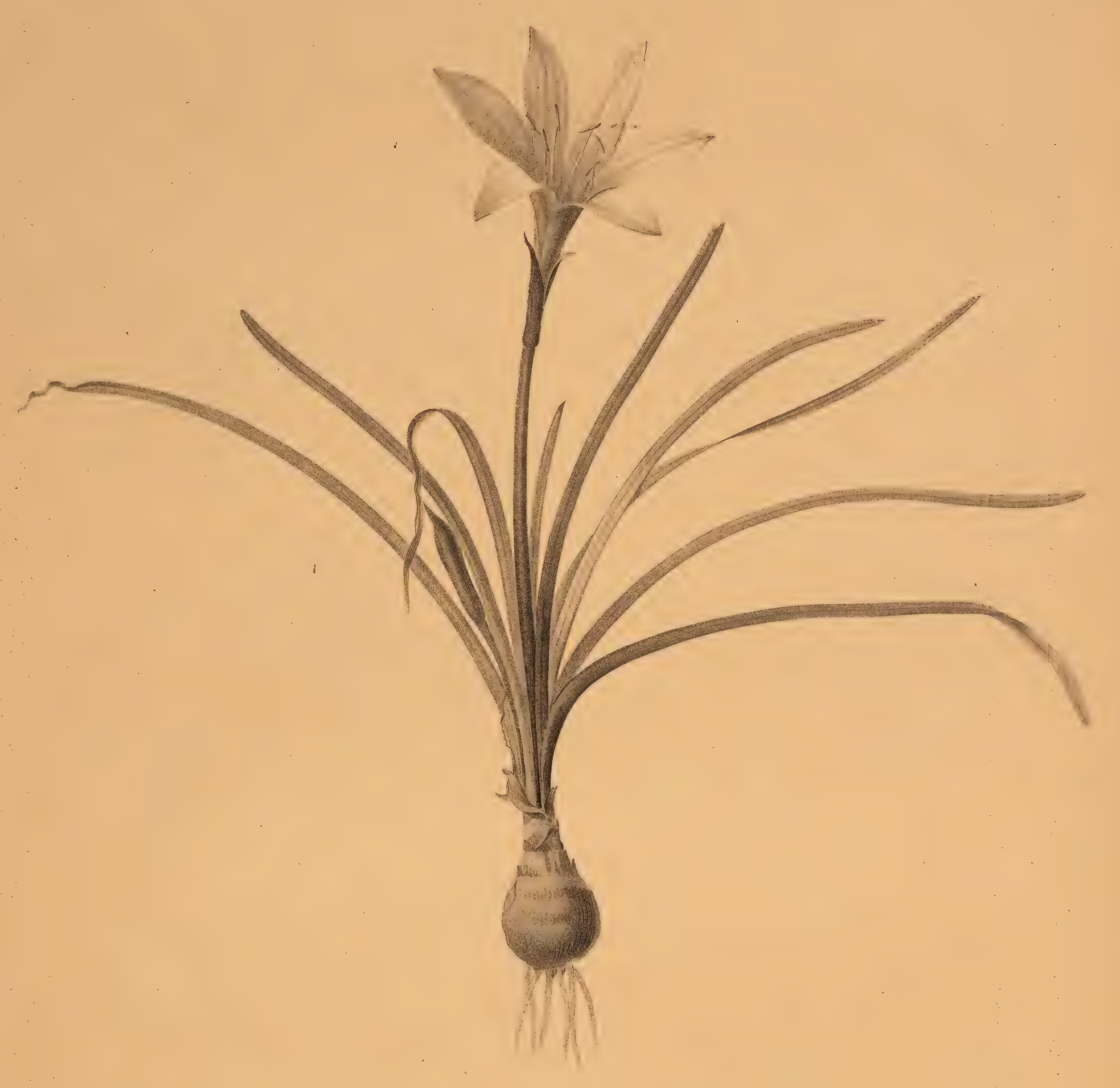

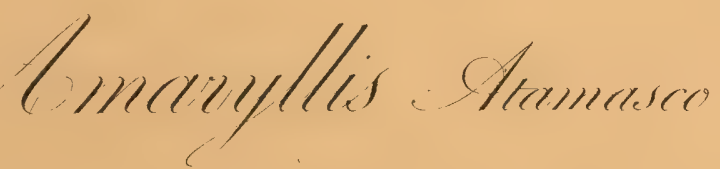

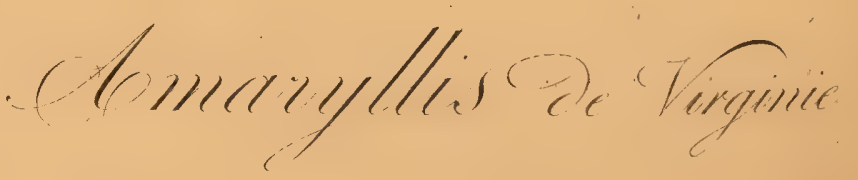






\title{
AMARYLLIS EQUESTRIS.
}

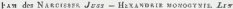

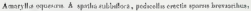

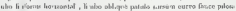

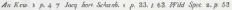

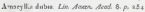

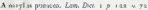

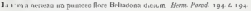

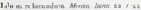

\section{WAEILLIS EOUESTRE}

\section{DKS.GRIfr:0X}

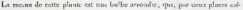

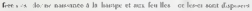

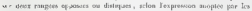

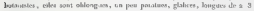
decesens.

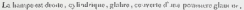

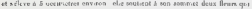

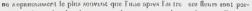

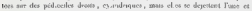

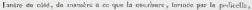

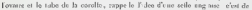

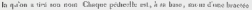

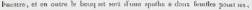

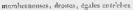

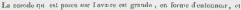

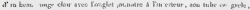

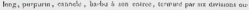

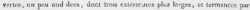

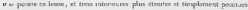

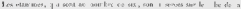

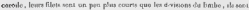

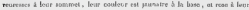




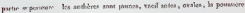
tail anc sw phater

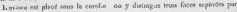

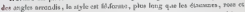

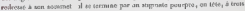

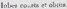

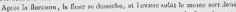

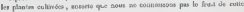
plarnis

\section{5 T 0 it $\mathrm{F}$}

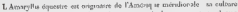

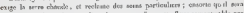

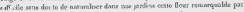

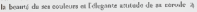

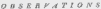

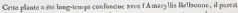

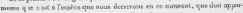

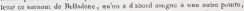

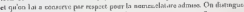

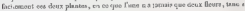

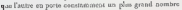




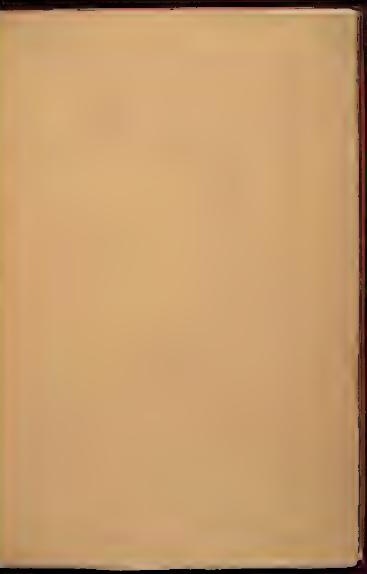




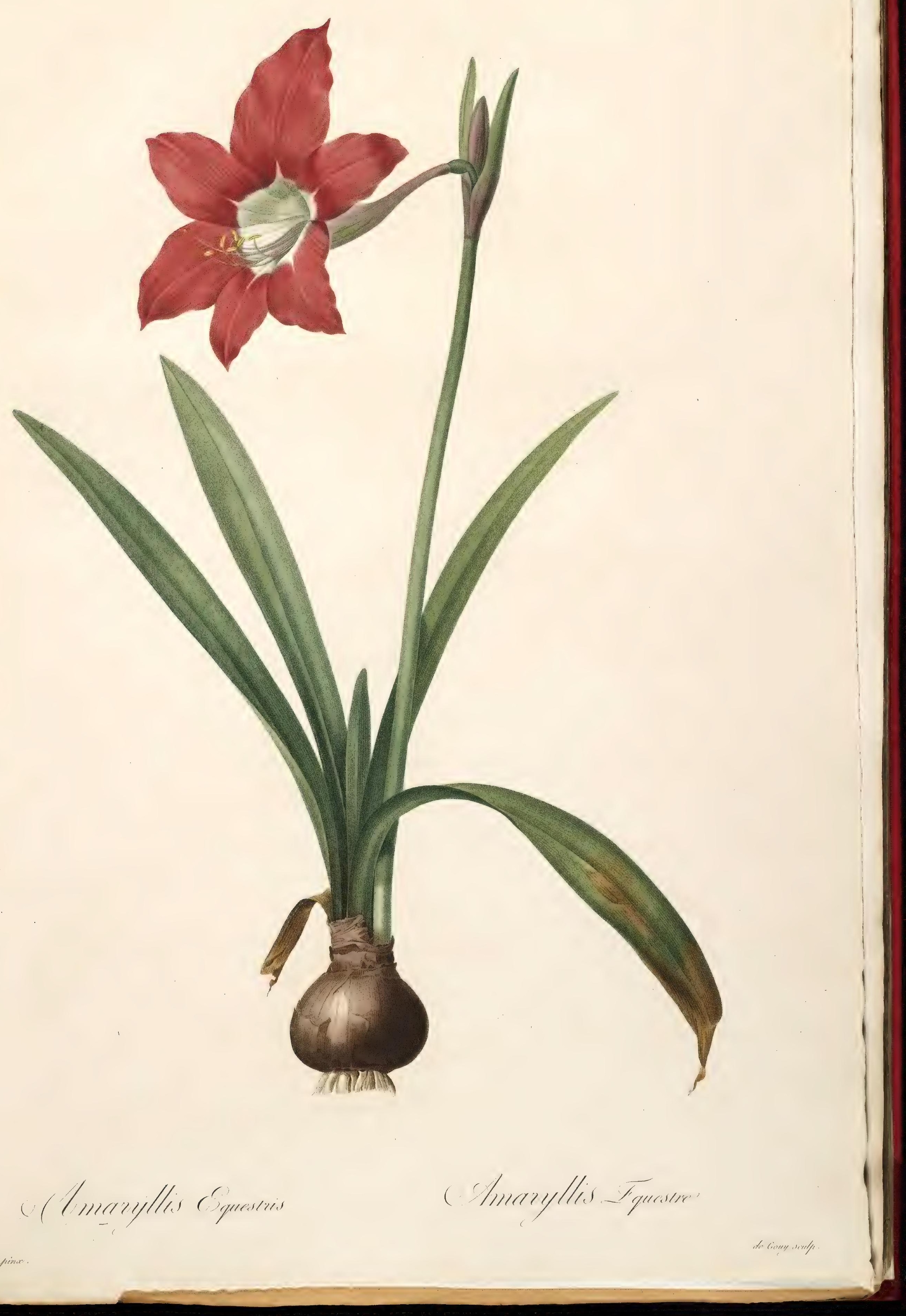





\section{AIARYLLIS SARNIENSTS.}

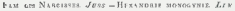

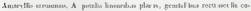

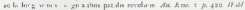
yृeny $=0$ if 5 ,

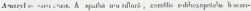

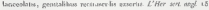

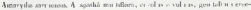

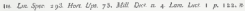

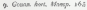

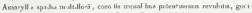

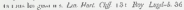

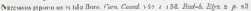

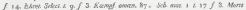
Oras. a. p. S.Sy

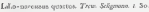

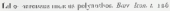

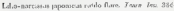

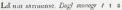

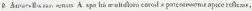

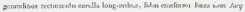

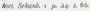

\section{IIAHULIS GLERMESIE Y Y}

\section{U B 5 C I T T TO N}

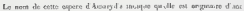

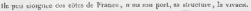

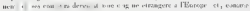

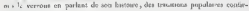

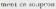

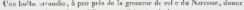

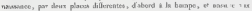

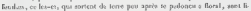

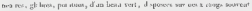




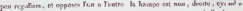

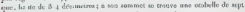

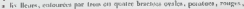

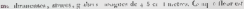

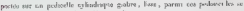

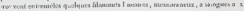
binitio

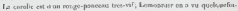

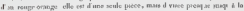

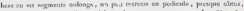

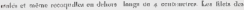

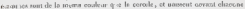

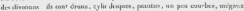

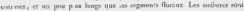

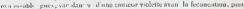

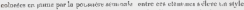

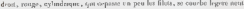

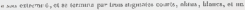
pou lietions

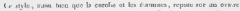

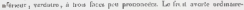
inre: Aas mas procis

\section{HISTOIRE}

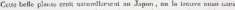

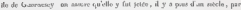

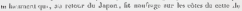

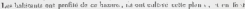

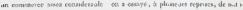

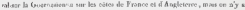

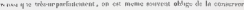

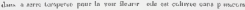

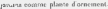

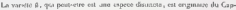

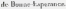



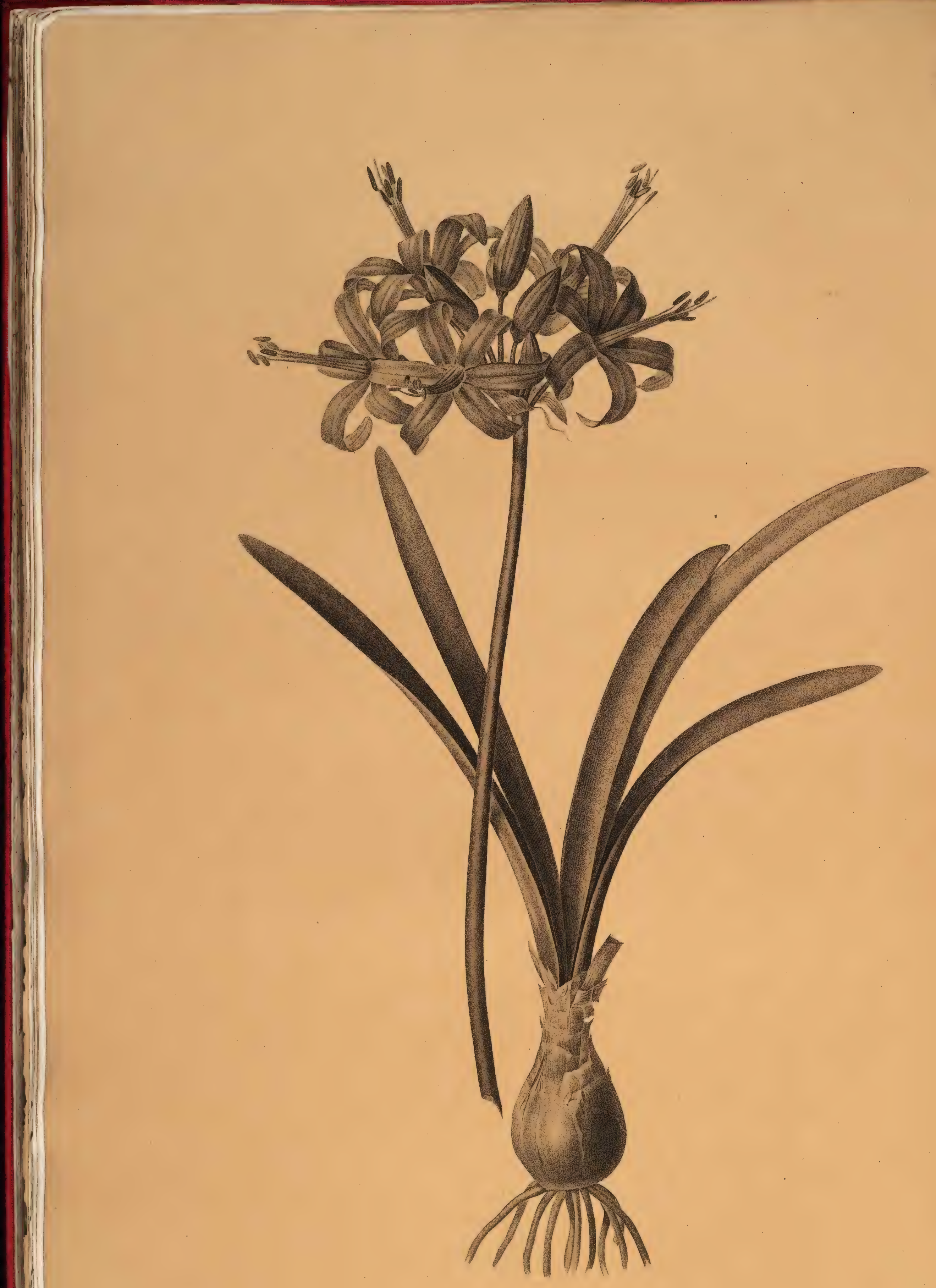

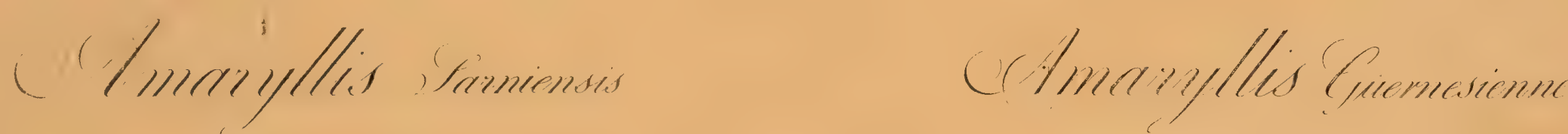




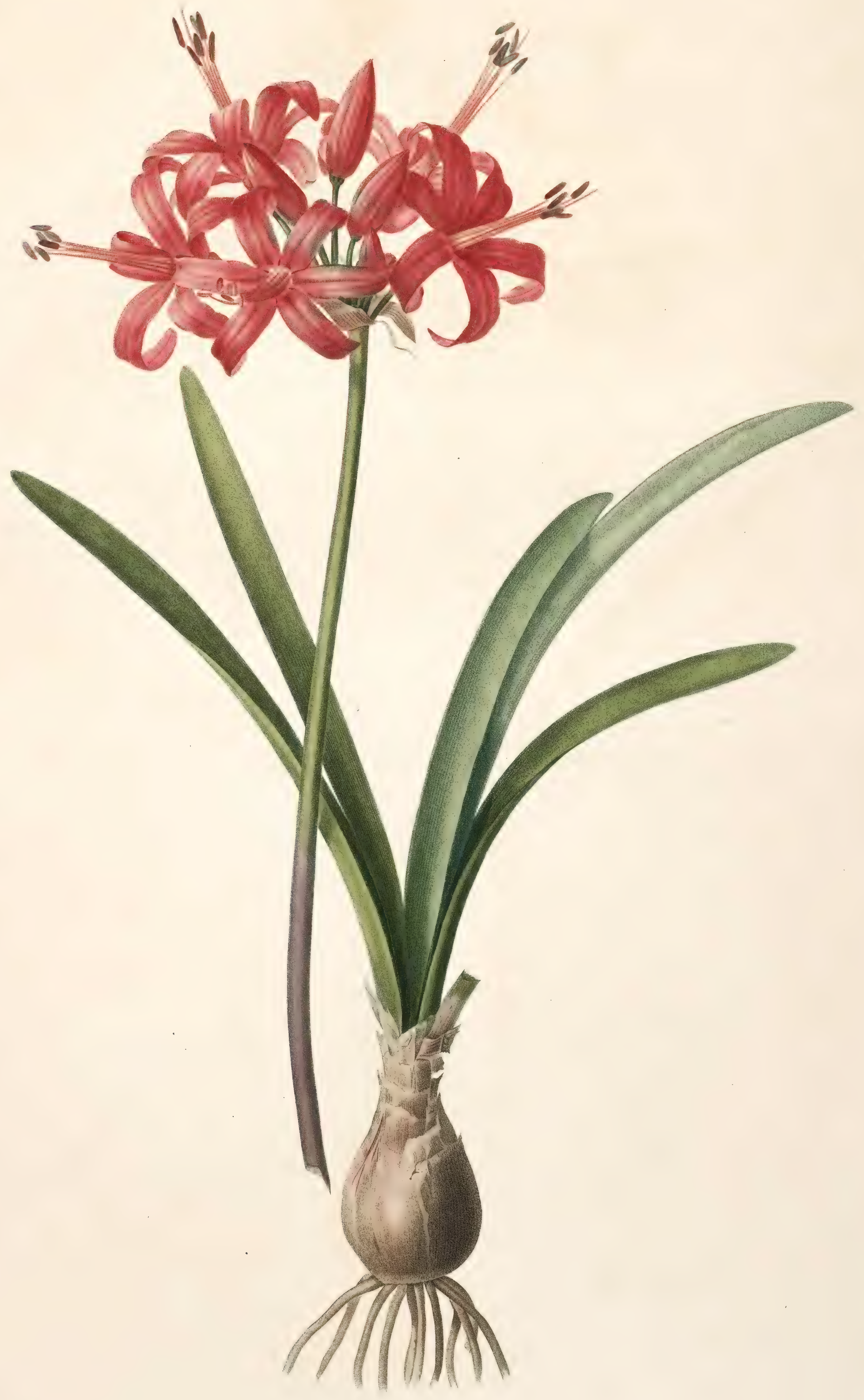

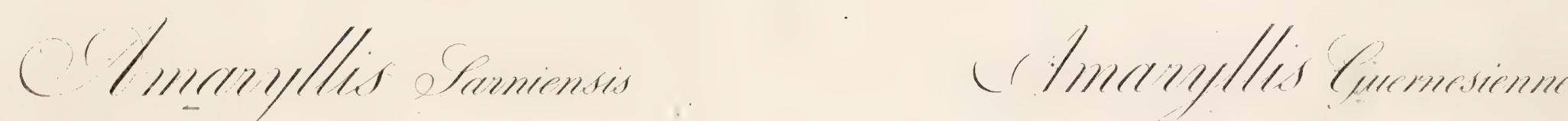




\section{IXIA LONGIFLORA.}

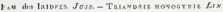

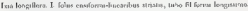

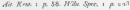

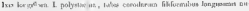

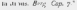

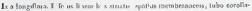

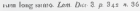

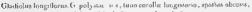

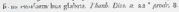

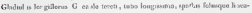
maen wora. Les espor got

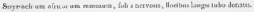
bing ain in

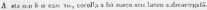

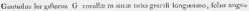

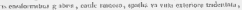

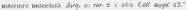

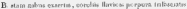

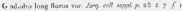

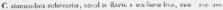

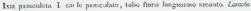
Des, p. $26,6 x+10$

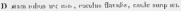

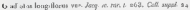

\section{IXIA $A$ LOFCLE ELECIR.}

\section{E CRTPTION}

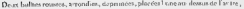

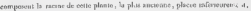

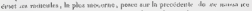

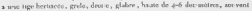

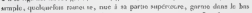

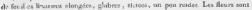

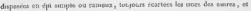




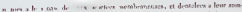

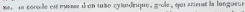

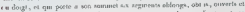

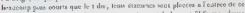

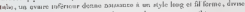

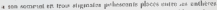

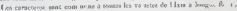

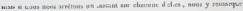

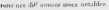

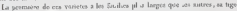

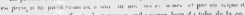

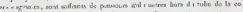

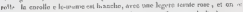

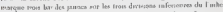

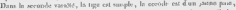

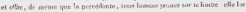

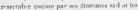

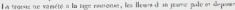

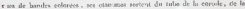

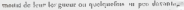

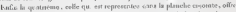

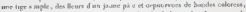

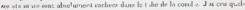

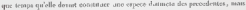

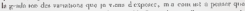

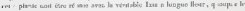

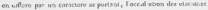

\section{A 5 I 0 ( 8 \&}

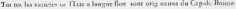

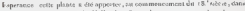

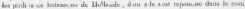
de rigunope 2

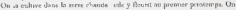
he at $n+$ al $\quad$ that

\section{XPLICATIOX DE LA PLAXCAS.}

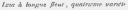

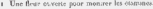

1. $\mathbf{L}=\boldsymbol{w}$ it 


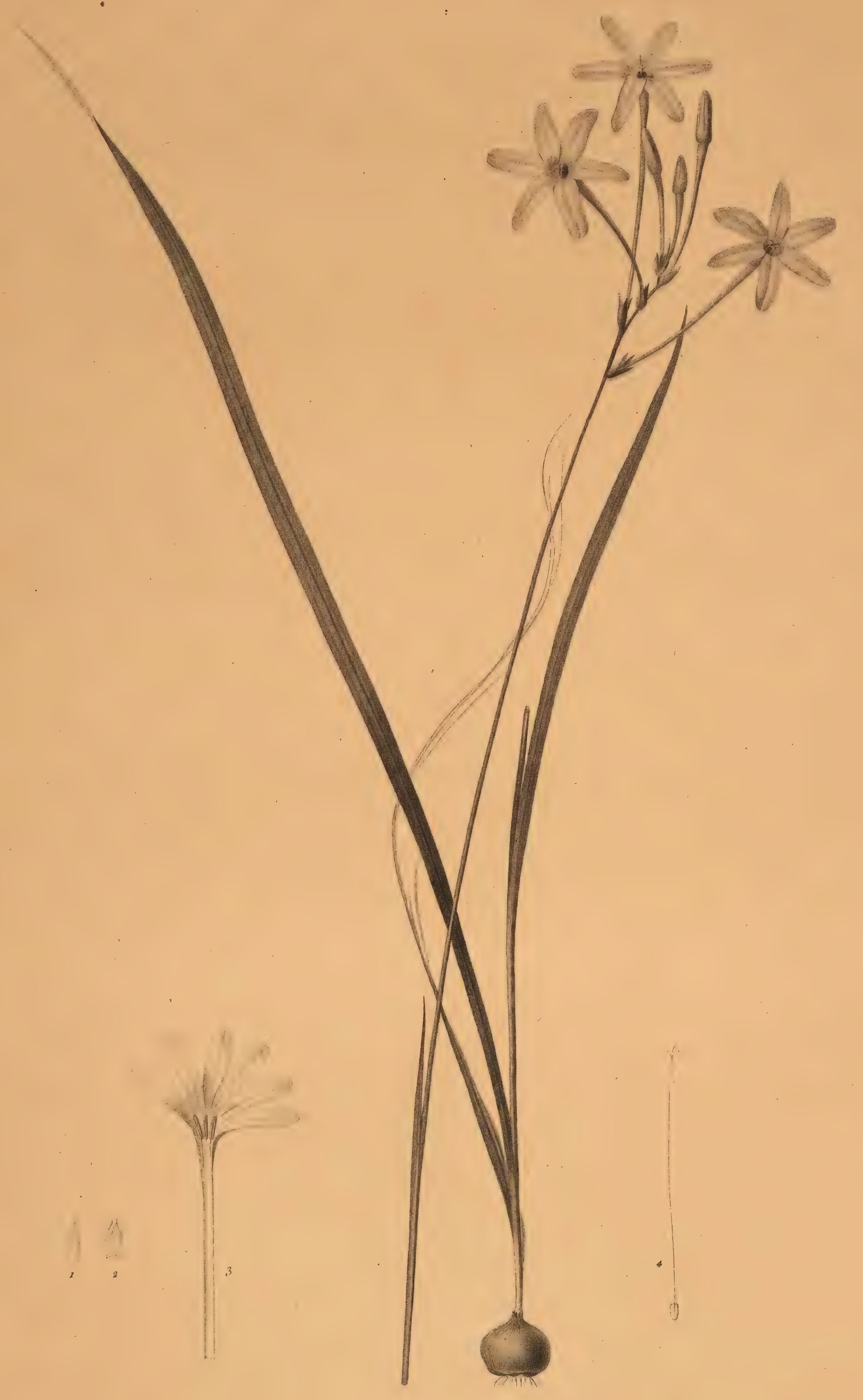

- Sxia Songiform Disia à longue Seur. 


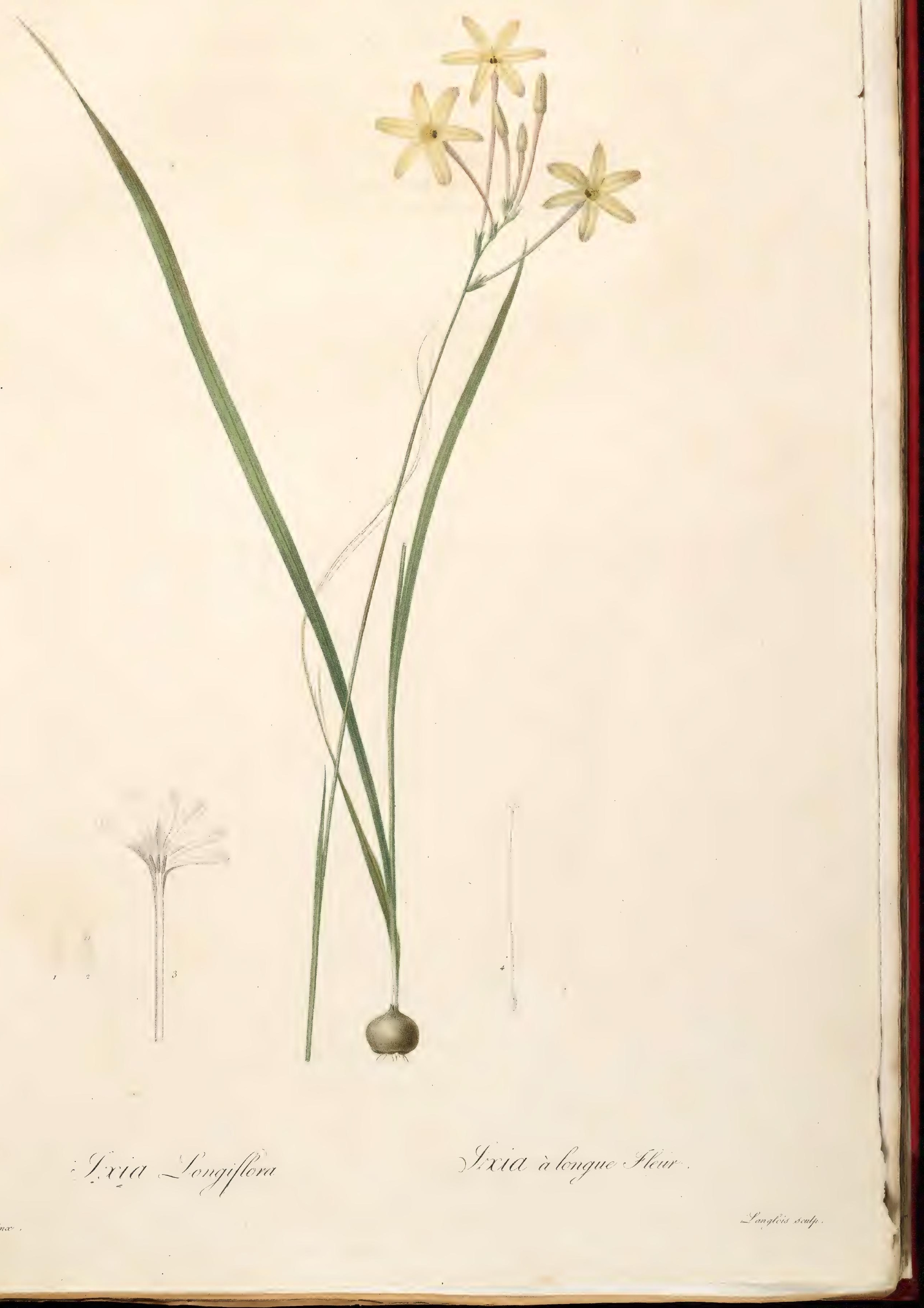




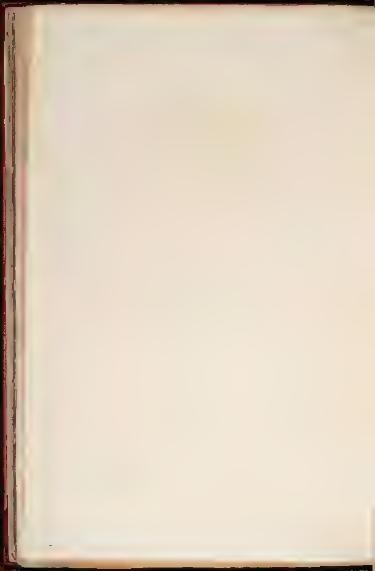




\title{
GLAD1OLUS TRISTIS.
}

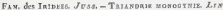

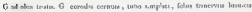

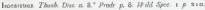

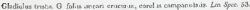

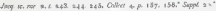

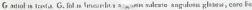

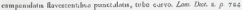
Dowe a sir

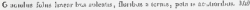
Lavo., a. 6 four 6. 235 \&. I

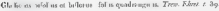

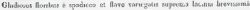

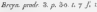

\section{GIAYEUL TAISTF.}

\section{DEमCRIP TIO X}

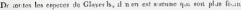

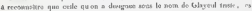

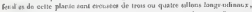

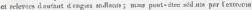

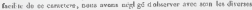

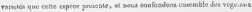
d ilerenta

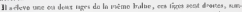

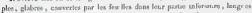

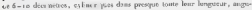

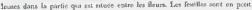

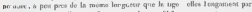

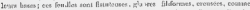

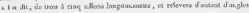
nxiluiks

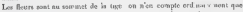

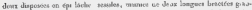

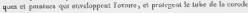




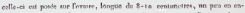

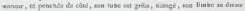

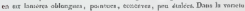

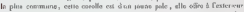

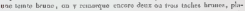

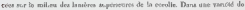

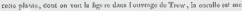

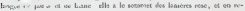

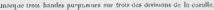

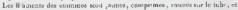

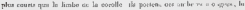

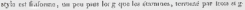

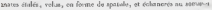

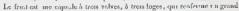

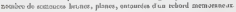

\section{$\pi 1 \& \mathrm{~T} 01 \mathrm{~h} x$}

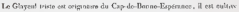

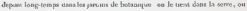
it fiscuir a i piracaids

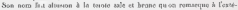

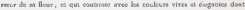
la plapart ax Glageule wrot decerce 



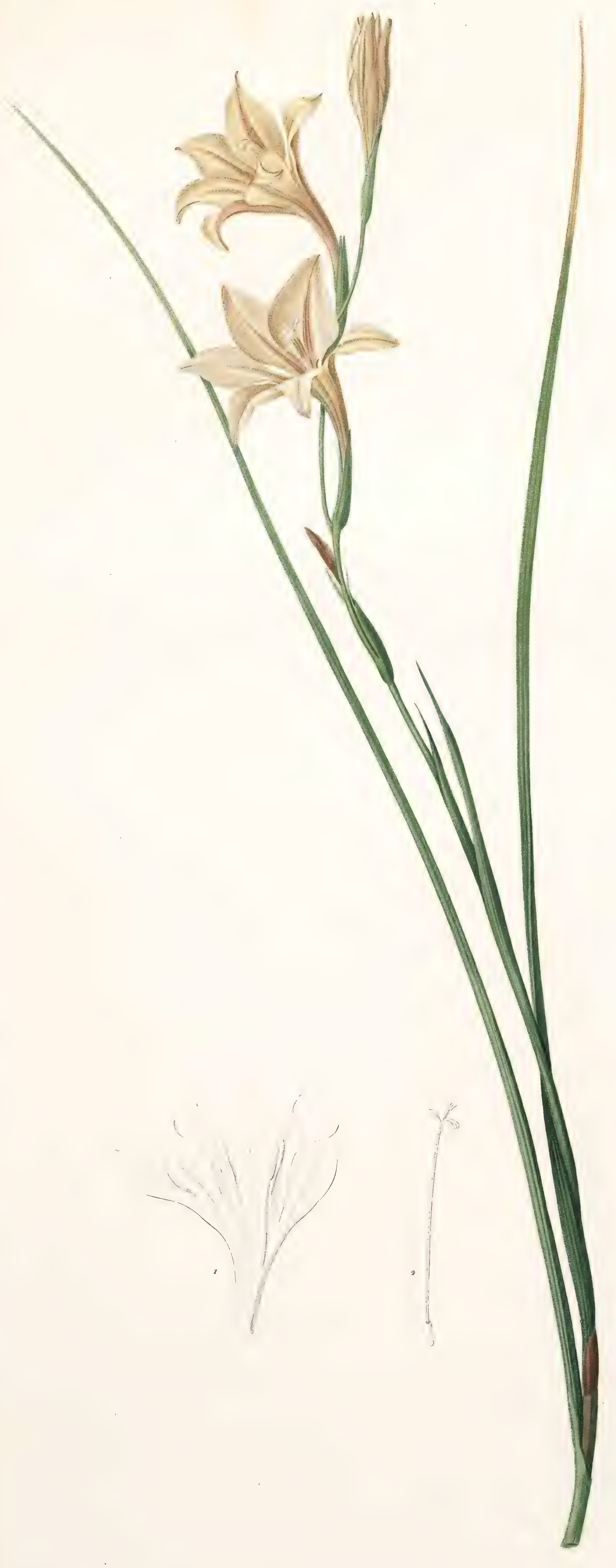

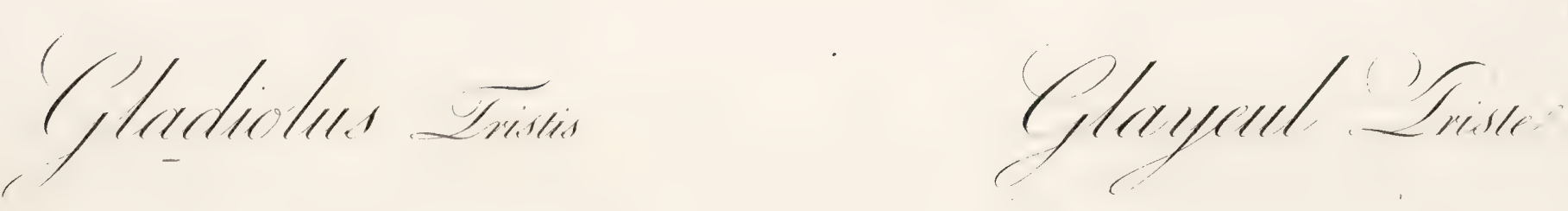





\title{
GLADIOLUS CUSPIDATUS
}

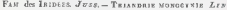

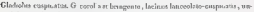

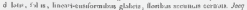

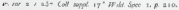

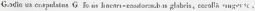

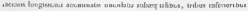

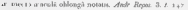

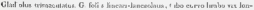

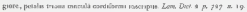

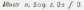

\section{GLAYELL EY POIXTE}

\section{DBSCH I T T T}

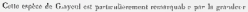

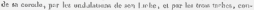

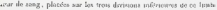

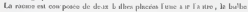

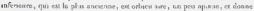

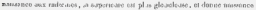

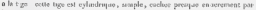

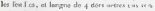

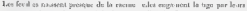

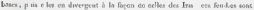

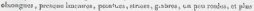

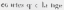

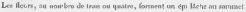

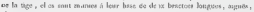

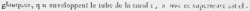

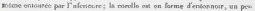

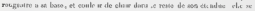

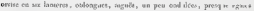

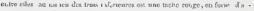

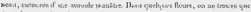

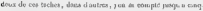




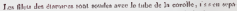

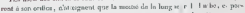

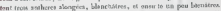

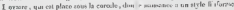

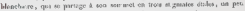

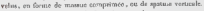

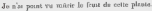

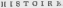

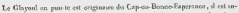

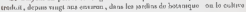

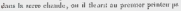

\section{IXFLICA I I $\mathrm{X}$ D D LA PLA R I T.}

\section{Le Phinto do griandion nahunethe}

- Le carofie ainene paje maniro los îsai aca,

5. Pra

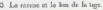




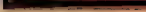




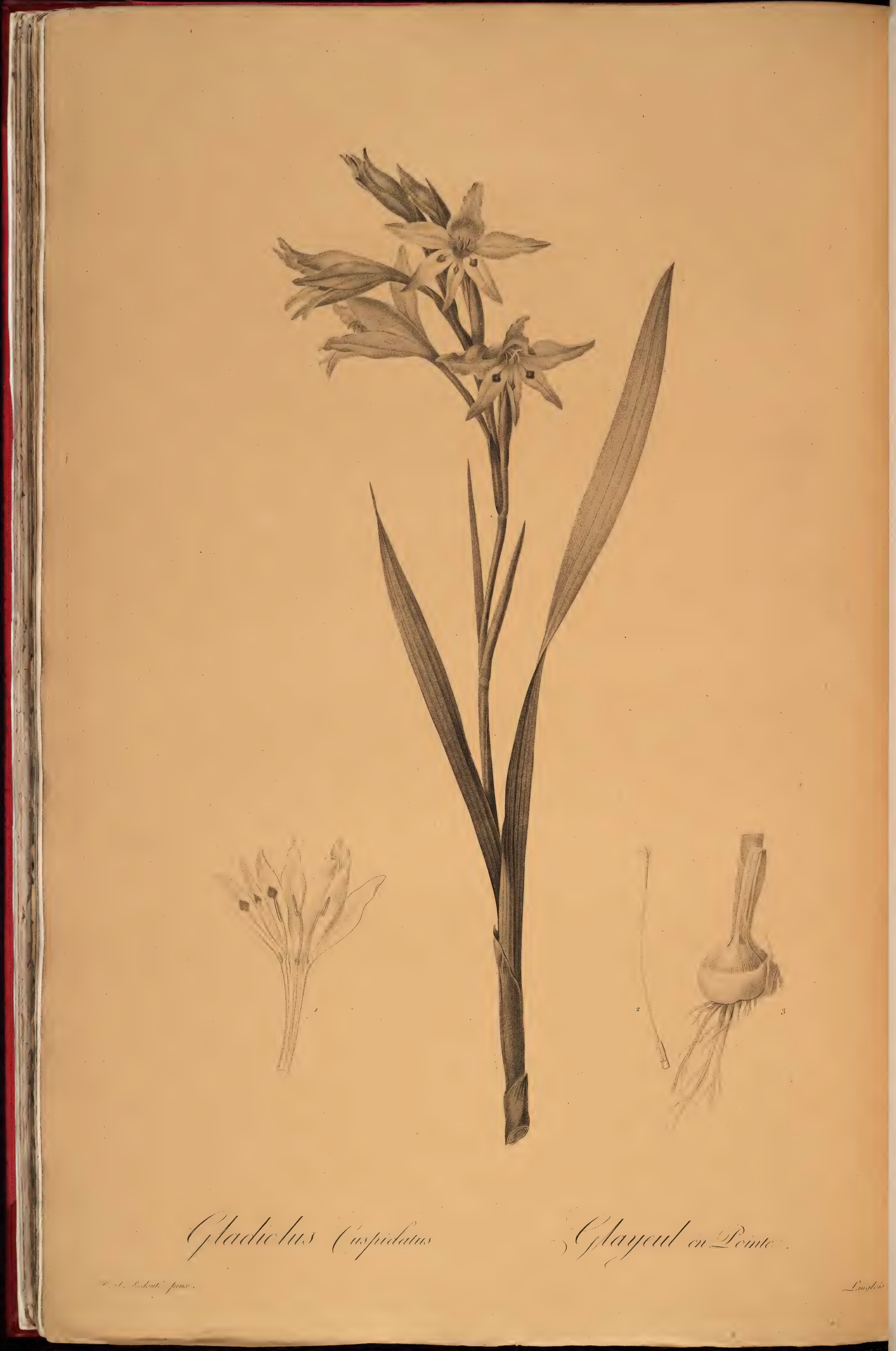




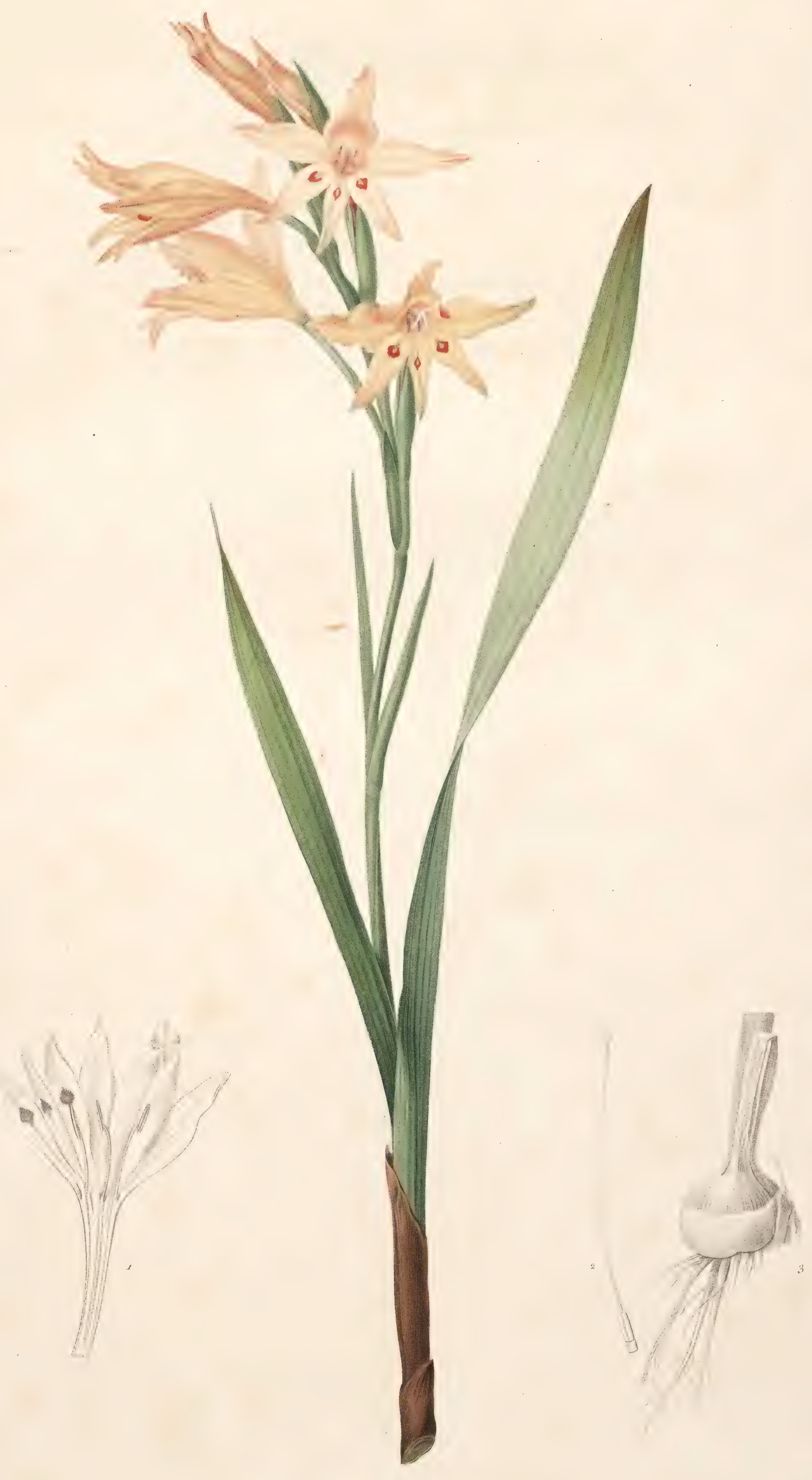

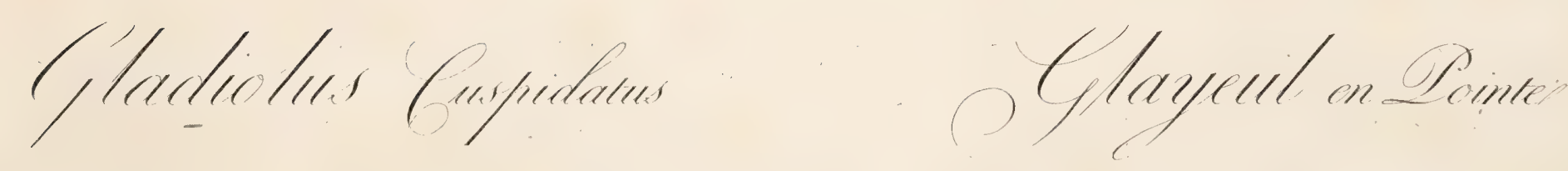





\section{TULIPA CLUSIANA.}

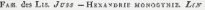

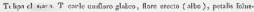

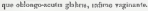

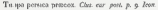

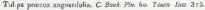

\section{TEIIPE DE L'ECIUSE}

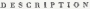

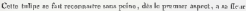

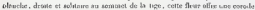

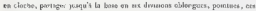

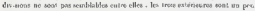

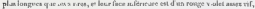

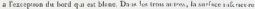

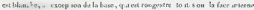

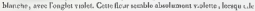
coet inas

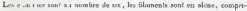

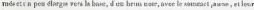

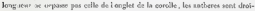

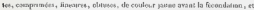

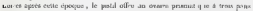

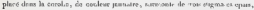

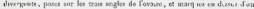
sil an probus

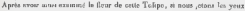

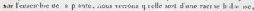

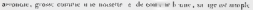

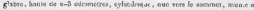

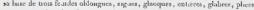

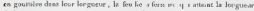

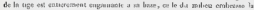

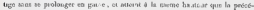

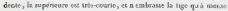




\section{7.}

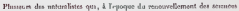

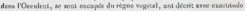

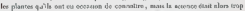

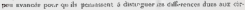

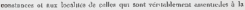

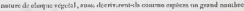

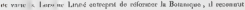

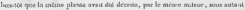

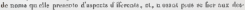

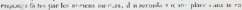

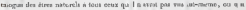

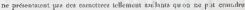

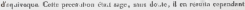

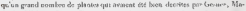

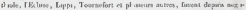

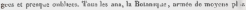

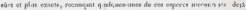

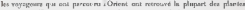

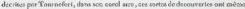

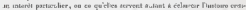

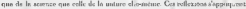

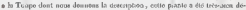

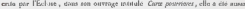

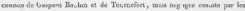
Boburin.

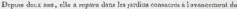

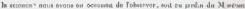

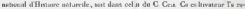

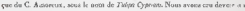

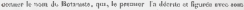

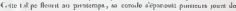

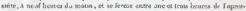
wi $\mathrm{T}$

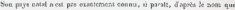

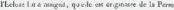

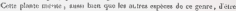
eolumes doms len juedins deocosent 



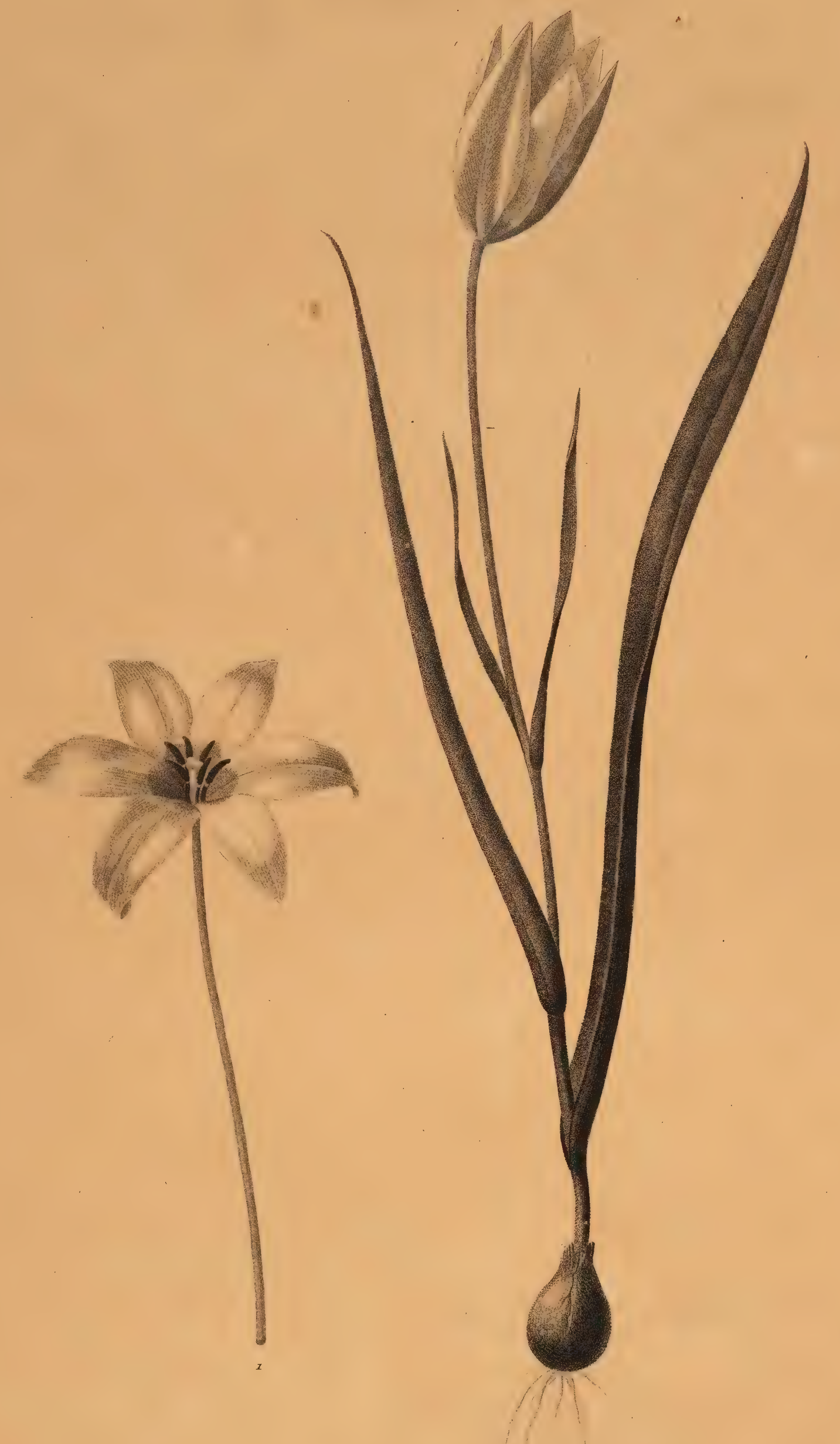

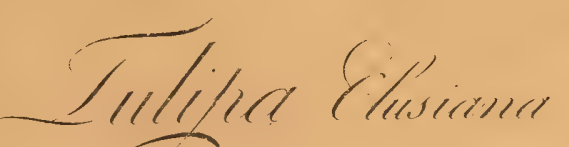

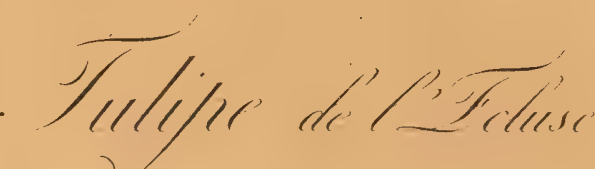




$$
y
$$




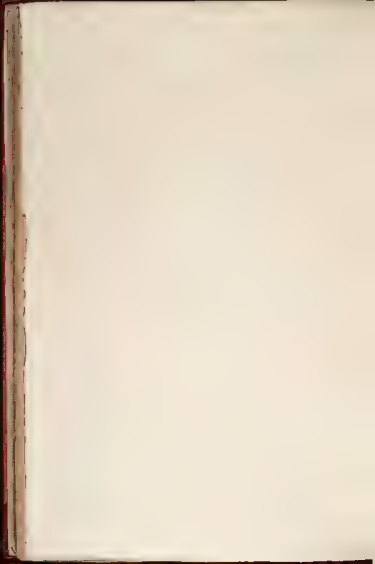




\title{
TULIPA CELSIANA.
}

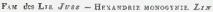

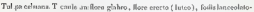

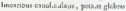

\section{TULIPL D E C E L S}

\section{I) E.S C R IPTI.ON}

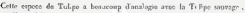

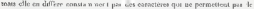

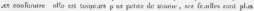

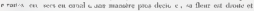

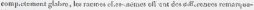

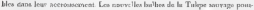

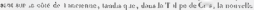

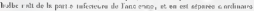

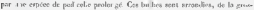

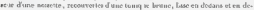

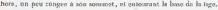

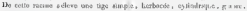

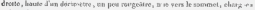

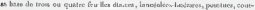

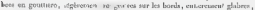

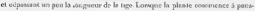

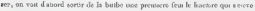

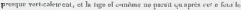

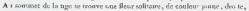

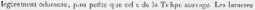

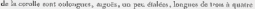

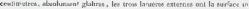

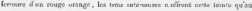

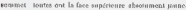

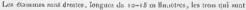

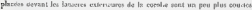

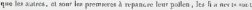

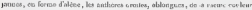
quat les filemernss 


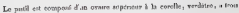

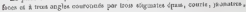

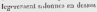

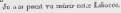

\section{I 5 TO I I I}

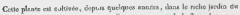

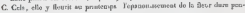
danl quclonese jeun.

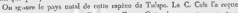

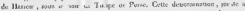

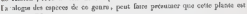
angousto d Oniese

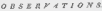

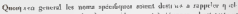

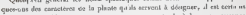

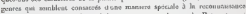

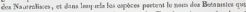

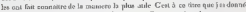

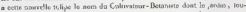

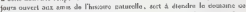

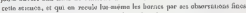

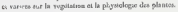





$$
Y
$$




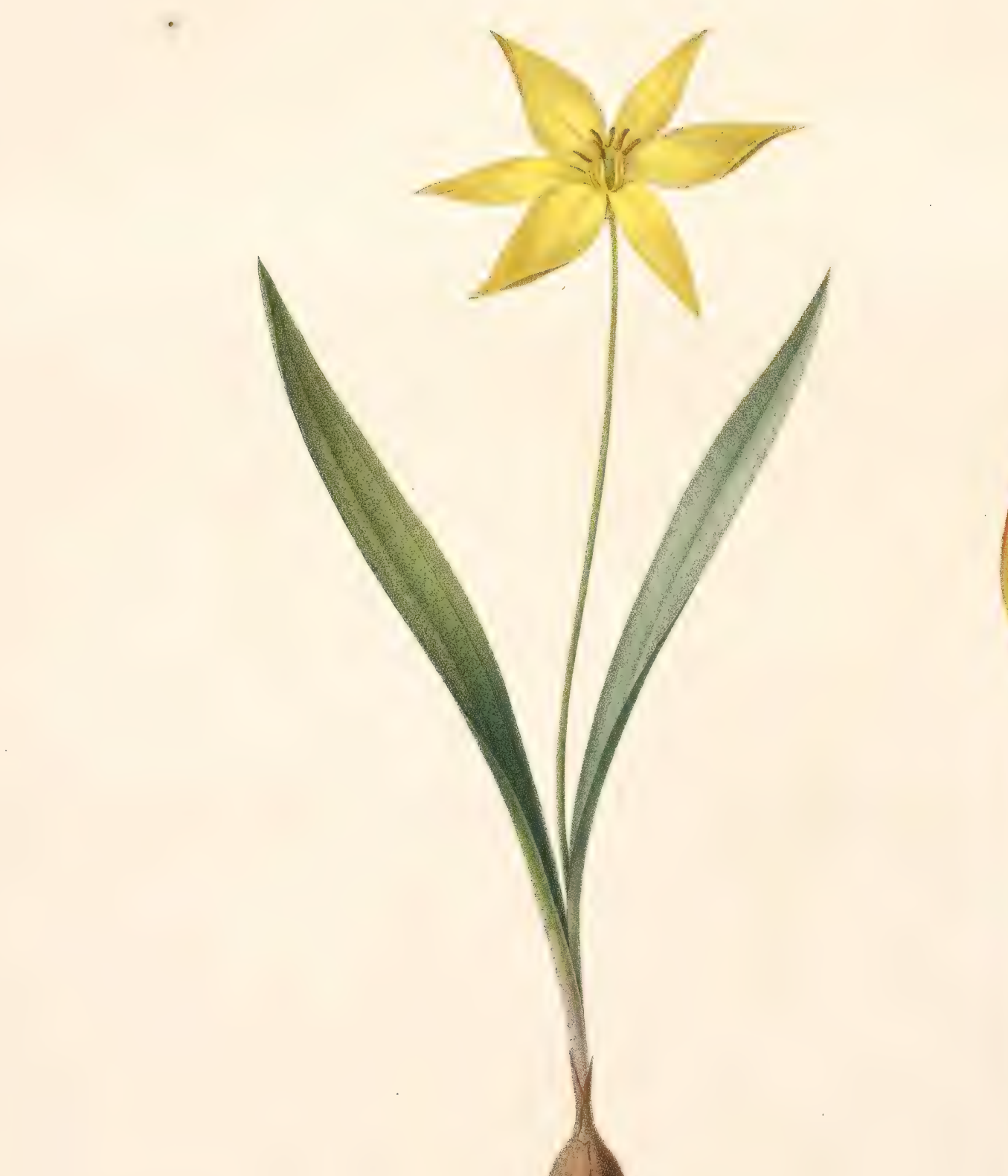




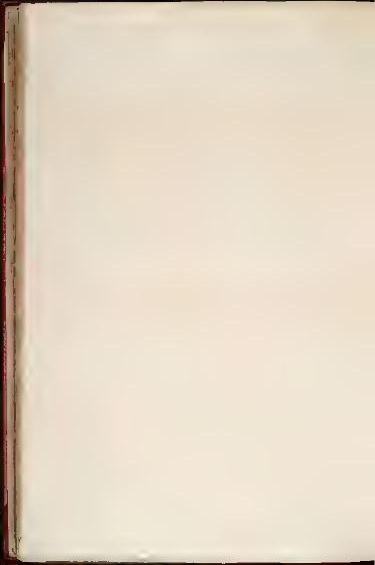




\title{
II EARANTIUS COCCINELS.
}

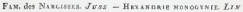

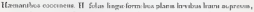

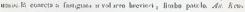

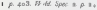

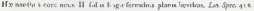

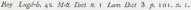

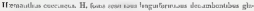

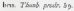

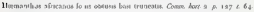

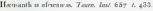

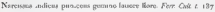

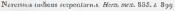

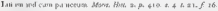

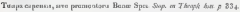

\section{AZ. U A THE F.CARLATR}

\section{E 5 E I P T T $\mathrm{x}$}

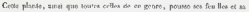

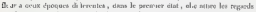

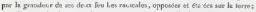

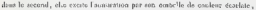

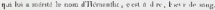

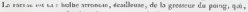

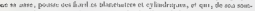

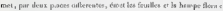

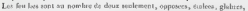

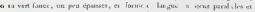

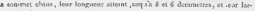

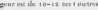

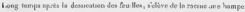

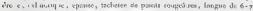

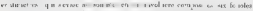

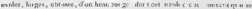

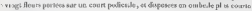
que limuliare 


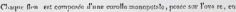

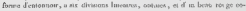

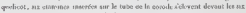

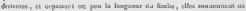

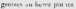

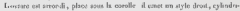

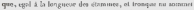

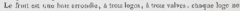

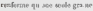

\section{I 5 T 0 IIIE}

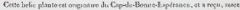

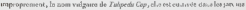

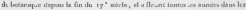

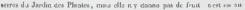

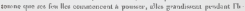

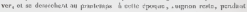

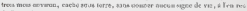

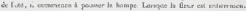

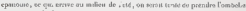

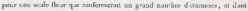
Iuvauore sacas li corola. 



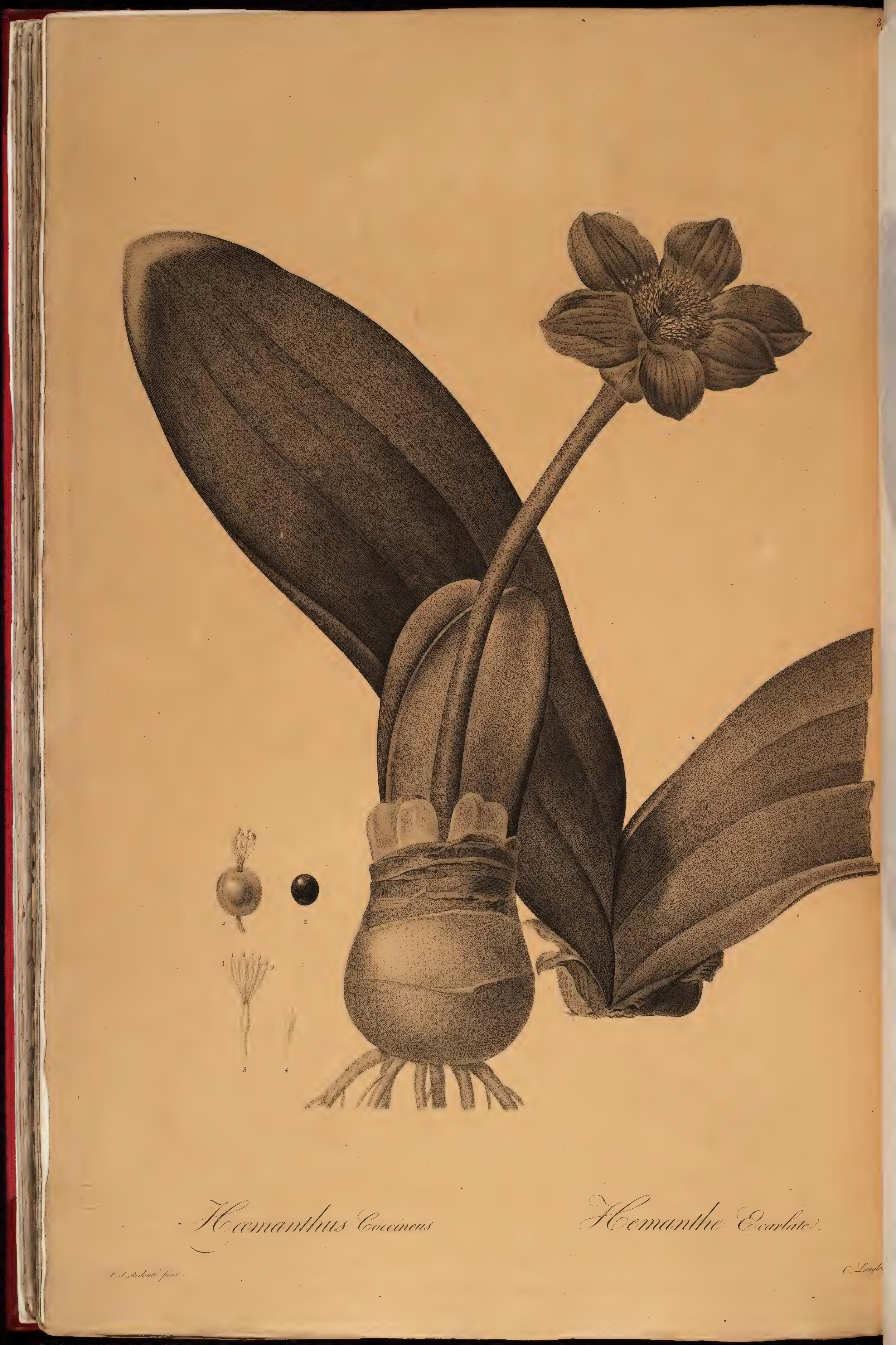




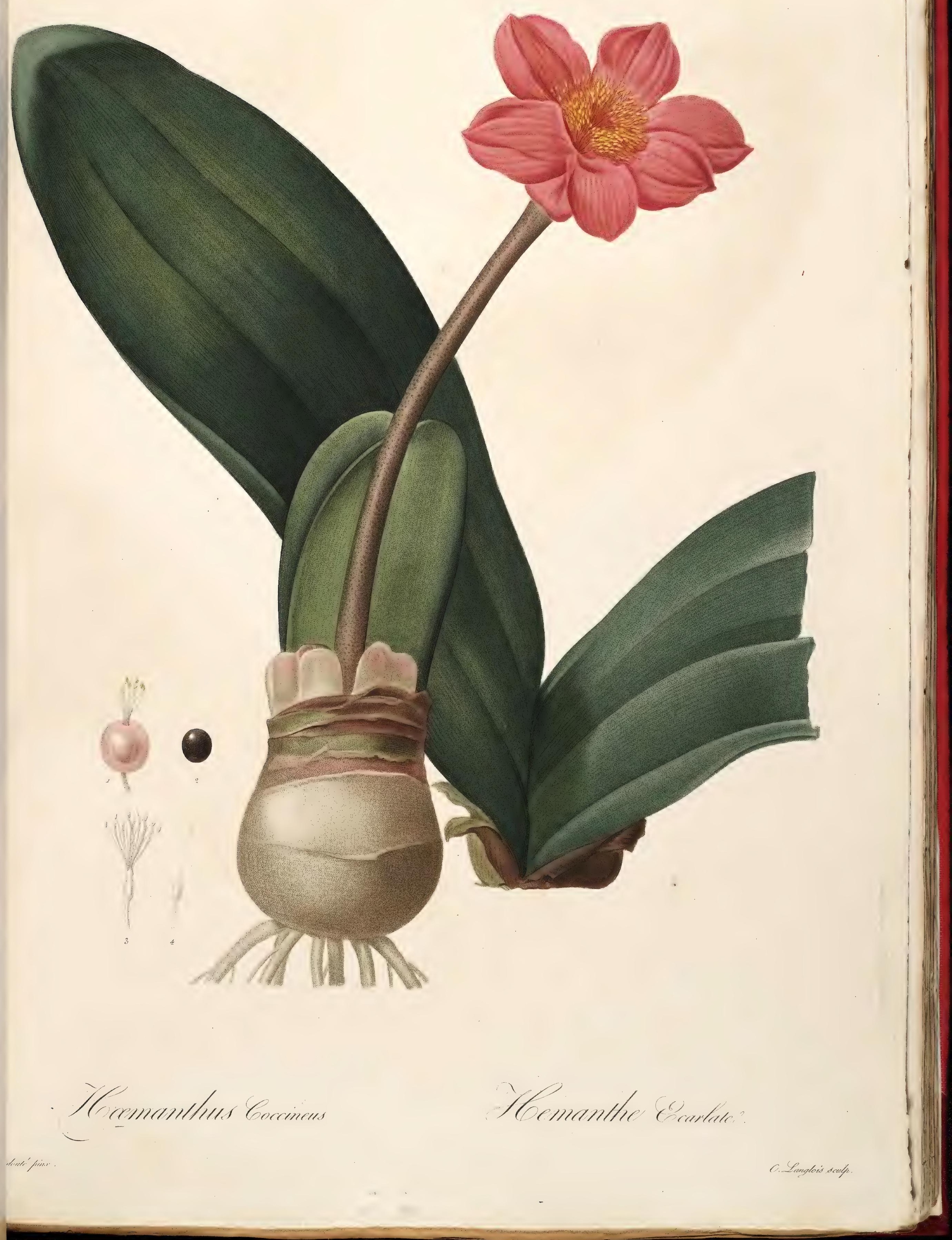





\section{ALSTROEMERIA LIGTU.}

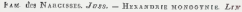

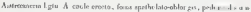

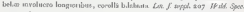
จ. 2k-105

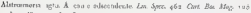
Lat Mavar. a sat $f$ i

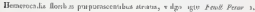
a. 7 ac ir 4

\section{ATSTRE.BFERIA LIGTE}

\section{I) $\mathrm{BSCR} 1 \mathrm{~T}$ T I O N}

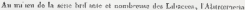

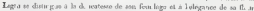

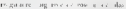

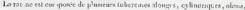

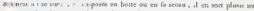

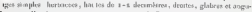

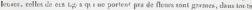

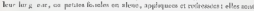

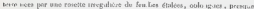

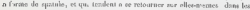

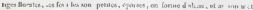

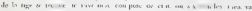

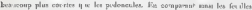

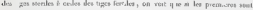

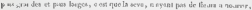

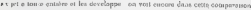

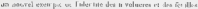

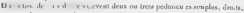

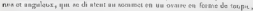

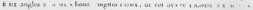

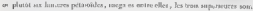
strvces olmon bo

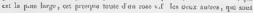




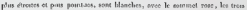

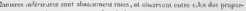

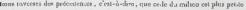
el plas poant at que is desi biweles.

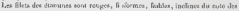

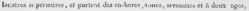

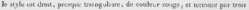

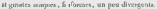

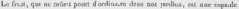

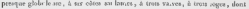
clasupo rwa frame fluevern pranes.

\section{$11+3 \% 0$ i $\mathrm{R}$}

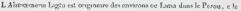

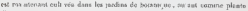

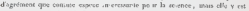

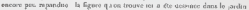

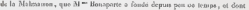

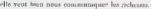

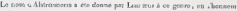

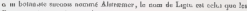

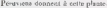

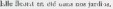





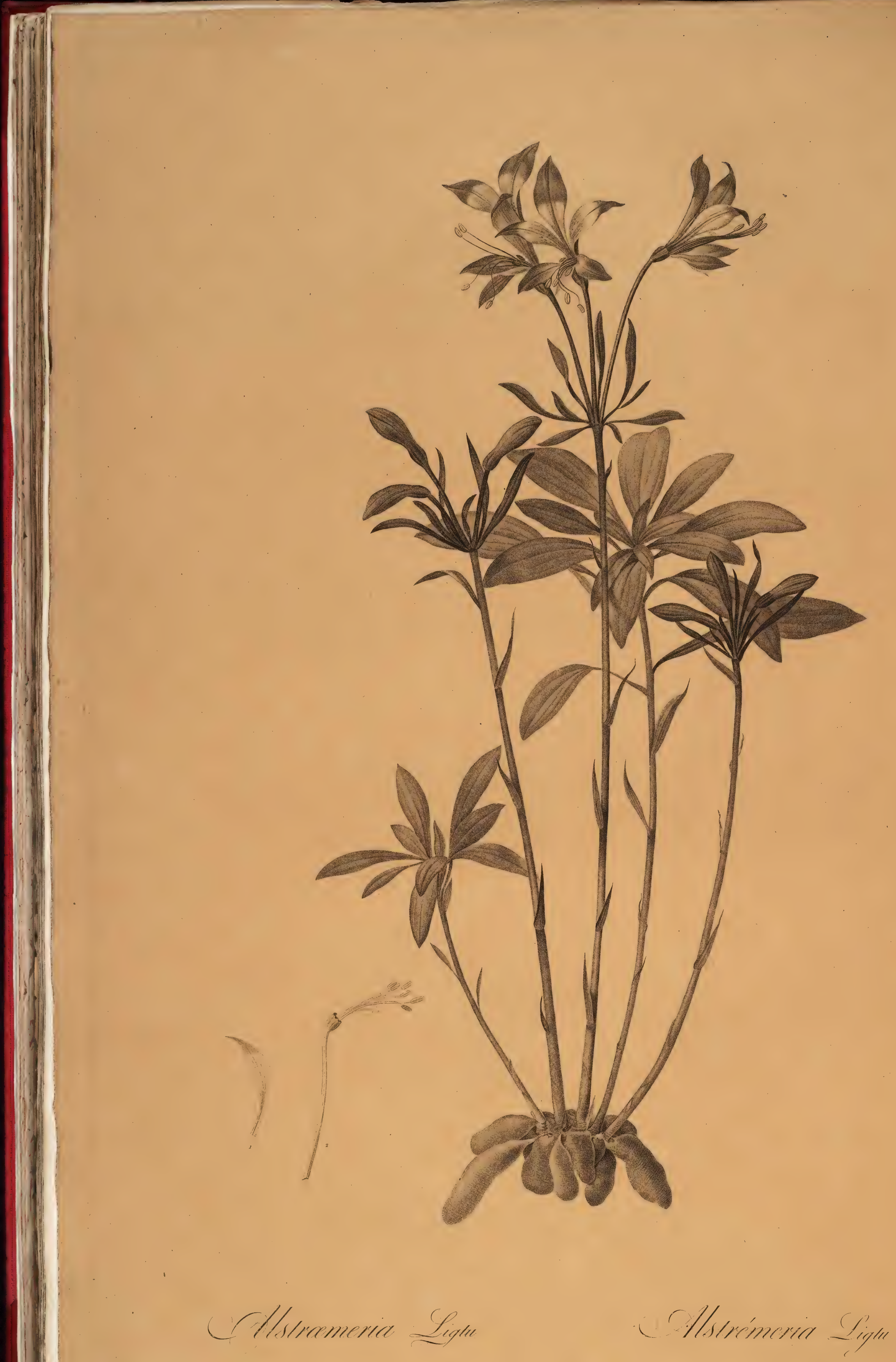




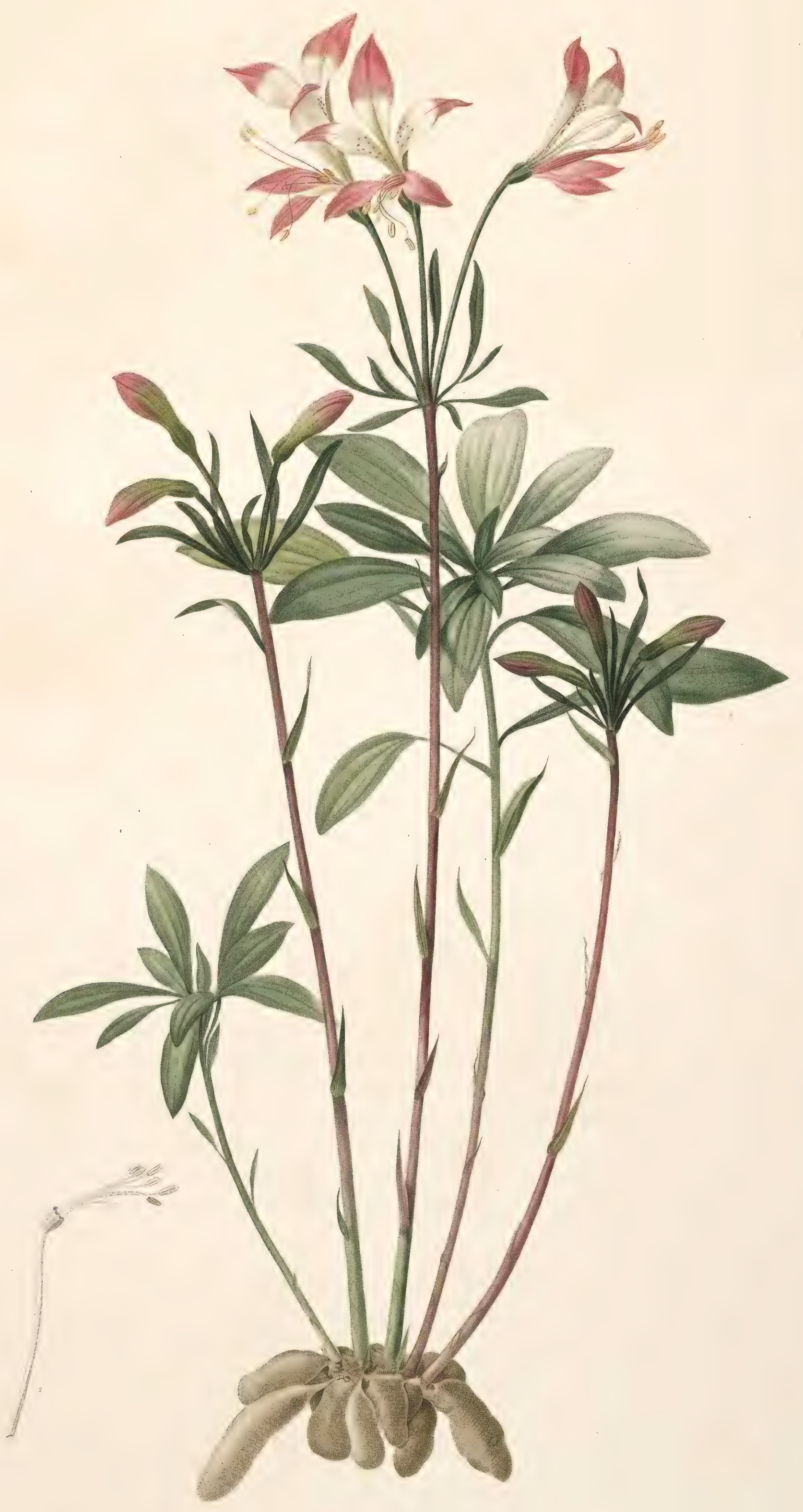

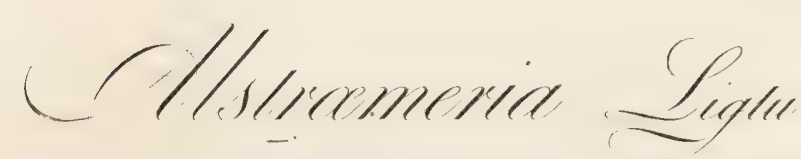

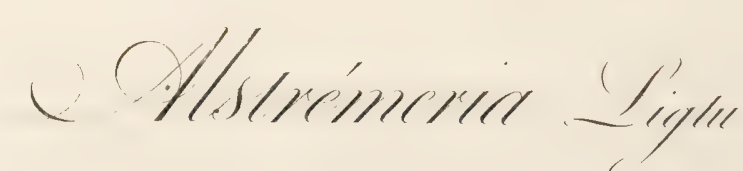





\title{
GALAXIA IXIEFLORA.
}

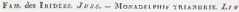

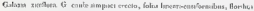

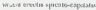

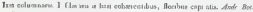
fivo , $903,210 \times 219$

\section{GALAXIA FLELR D'IXIA}

\section{ISCRIPTION}

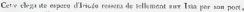

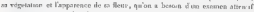

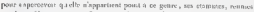

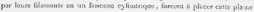

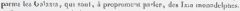

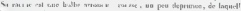

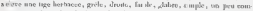

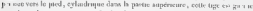

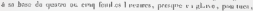

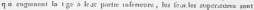

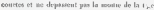

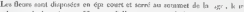

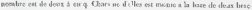

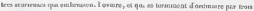

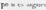

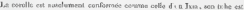

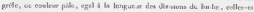

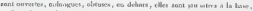

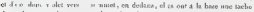

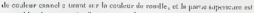

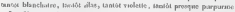

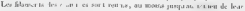

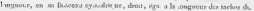

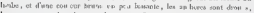

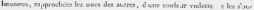

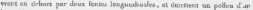
Li o blutars 


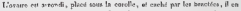

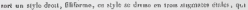

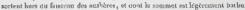

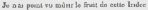

\section{I $50.1 \mathrm{TL}$}

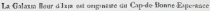

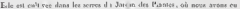

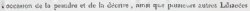
Hent newe renns dege prit.

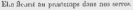

$$
\text { OBSERPATIONS }
$$

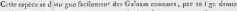

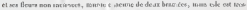

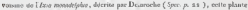

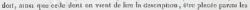

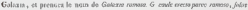

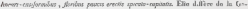

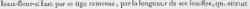

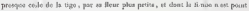

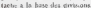

RXIIICATJOK DELA PLANC II L

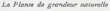

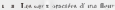

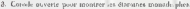

4 Lo paris

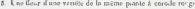






\section{VIEUSSEUXIA GLAVCOPIS.}

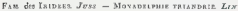

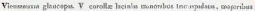

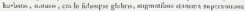

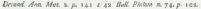

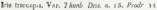

\section{MEUSSEUXIE A TACHLS BIELES}

\section{DESCA IPT 10 X}

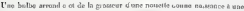

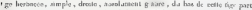

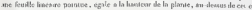

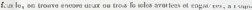

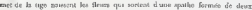

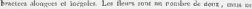

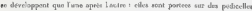

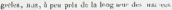

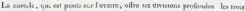

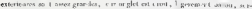

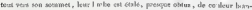

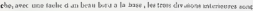

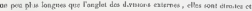

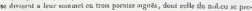

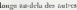

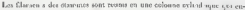

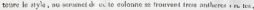

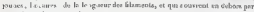
deox fened lengramiakes

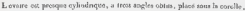

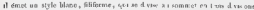

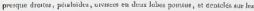
bords

\section{IS I I T I R}

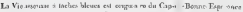

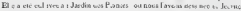




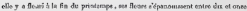

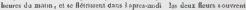
d des pourr doflecenle

$$
O D S \& F A T I O N=
$$

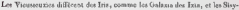

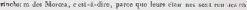

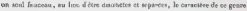

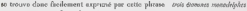

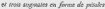

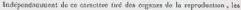

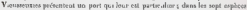

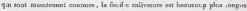

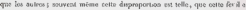

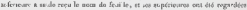
comme ile smaples fou llich

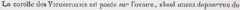

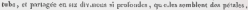

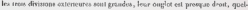

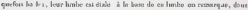

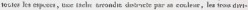

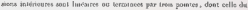

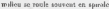

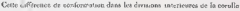

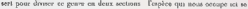

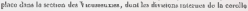
wonl decos gouiter

\section{IXPLICATTON DE LA PLAXCBE}

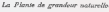

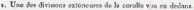

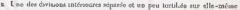
1 ad sairition.

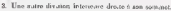

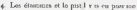

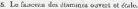

6. Lo prsil mes o ait. 



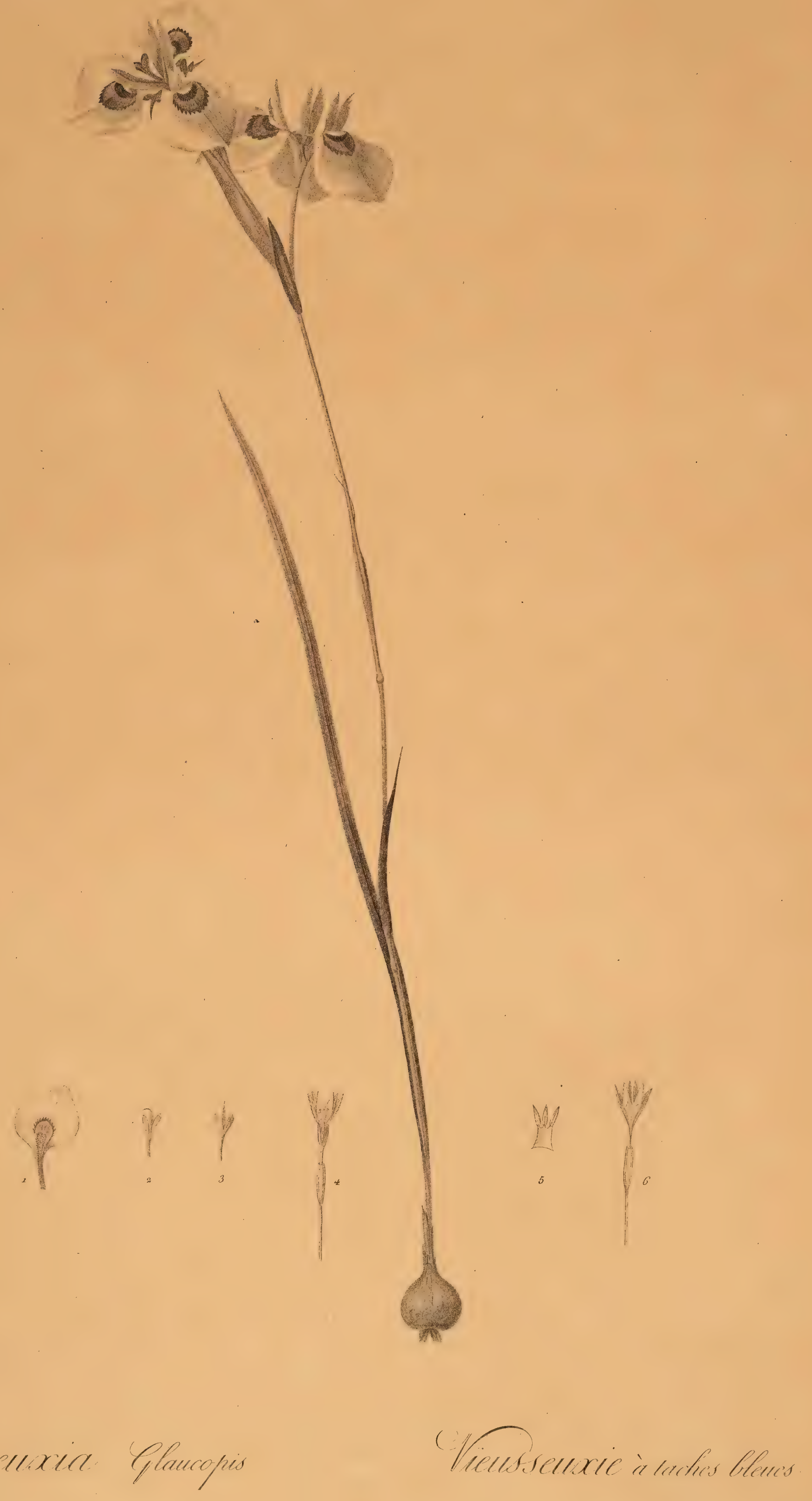




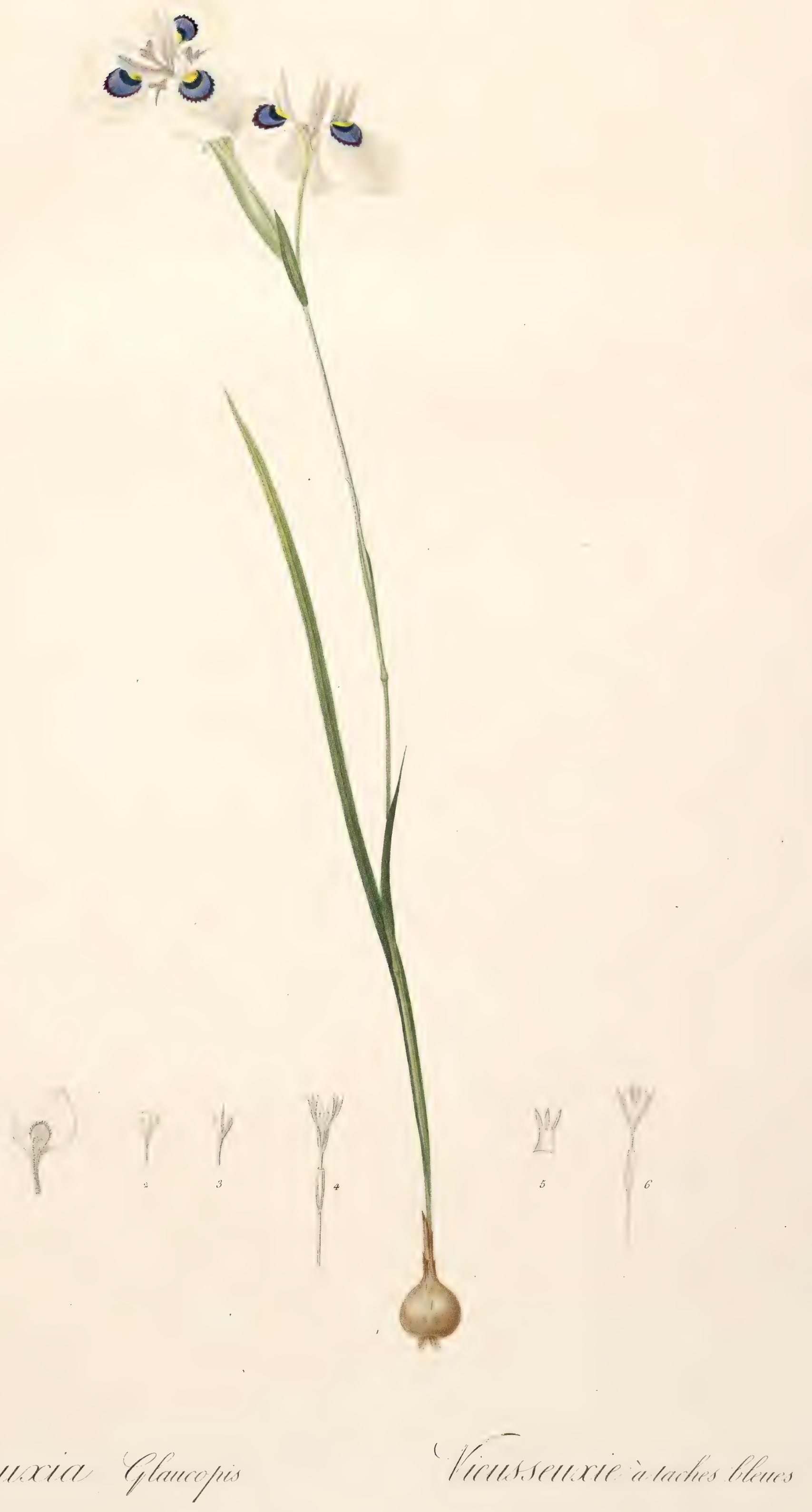

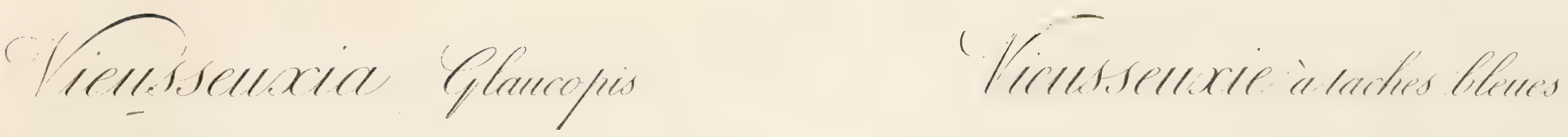





\title{
LIMODORLU TAXKER WILLE.
}

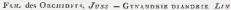

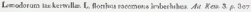

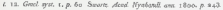

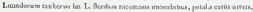

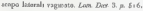

\section{LIATODOIE DE TISHERMILL}

\section{DEFTתTRTION}

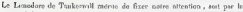

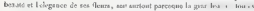

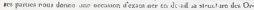

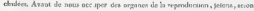

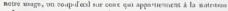

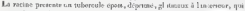

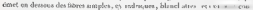

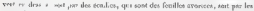

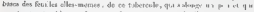

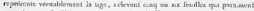

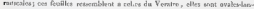

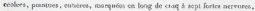

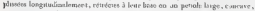

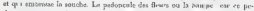

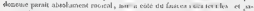

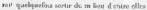

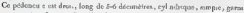

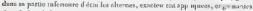

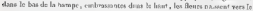

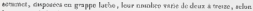

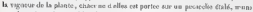

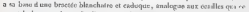

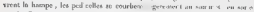

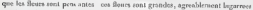

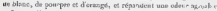

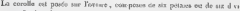

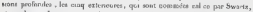

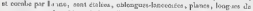

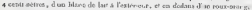

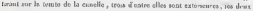




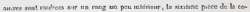

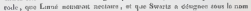

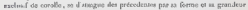

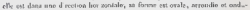

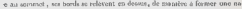

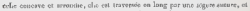

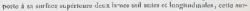

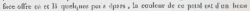

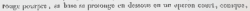

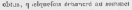

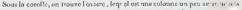

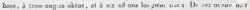

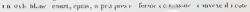

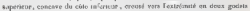

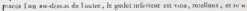

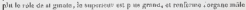

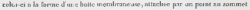

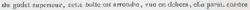

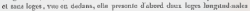

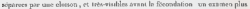

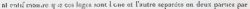

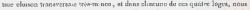

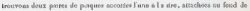

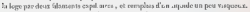

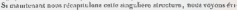

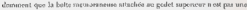

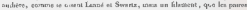

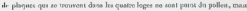

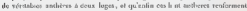

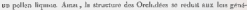

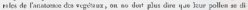

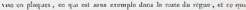

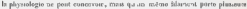

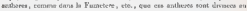

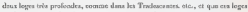

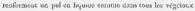

\section{I 5 T 10 . F}

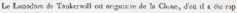
perts wa Arglit m

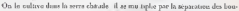

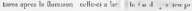






\title{
GLADIOLUS INCLIXATUS.
}

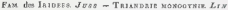

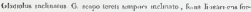

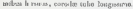

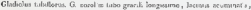

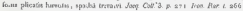

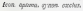

\section{GI, AYUL I CEINF}

\section{D $x$ a 1 I 1 T 19}

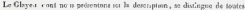

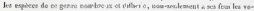

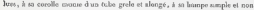

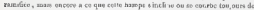

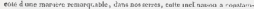

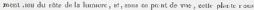

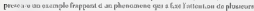

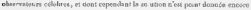

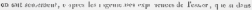

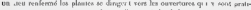

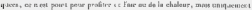

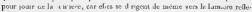
e. 16 per an mines

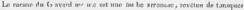

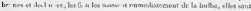

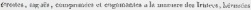

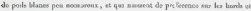

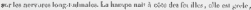

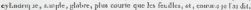

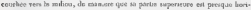
sonia o

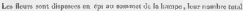

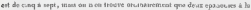

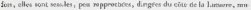

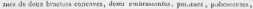

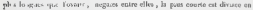
deus larreses 


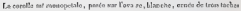

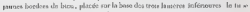

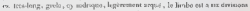

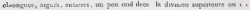

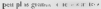

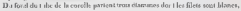

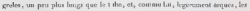

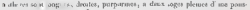

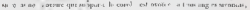

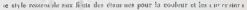

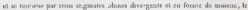

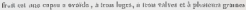

\section{I S I O I R B}

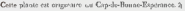

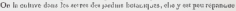

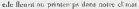

Dh la mulaplie do exguas

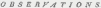

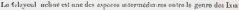

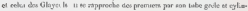

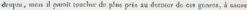

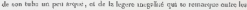
Af. wank de ver livatse

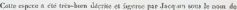

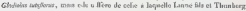

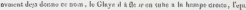

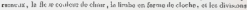

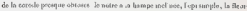

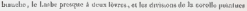

\section{EX P I C A T I O N D B LA E L A N H E}

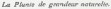

- La lorar-es in Girkure

2 La bractesa spenoura.

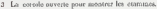

\& $\mathrm{Le}[\mathrm{iat}$ ! 


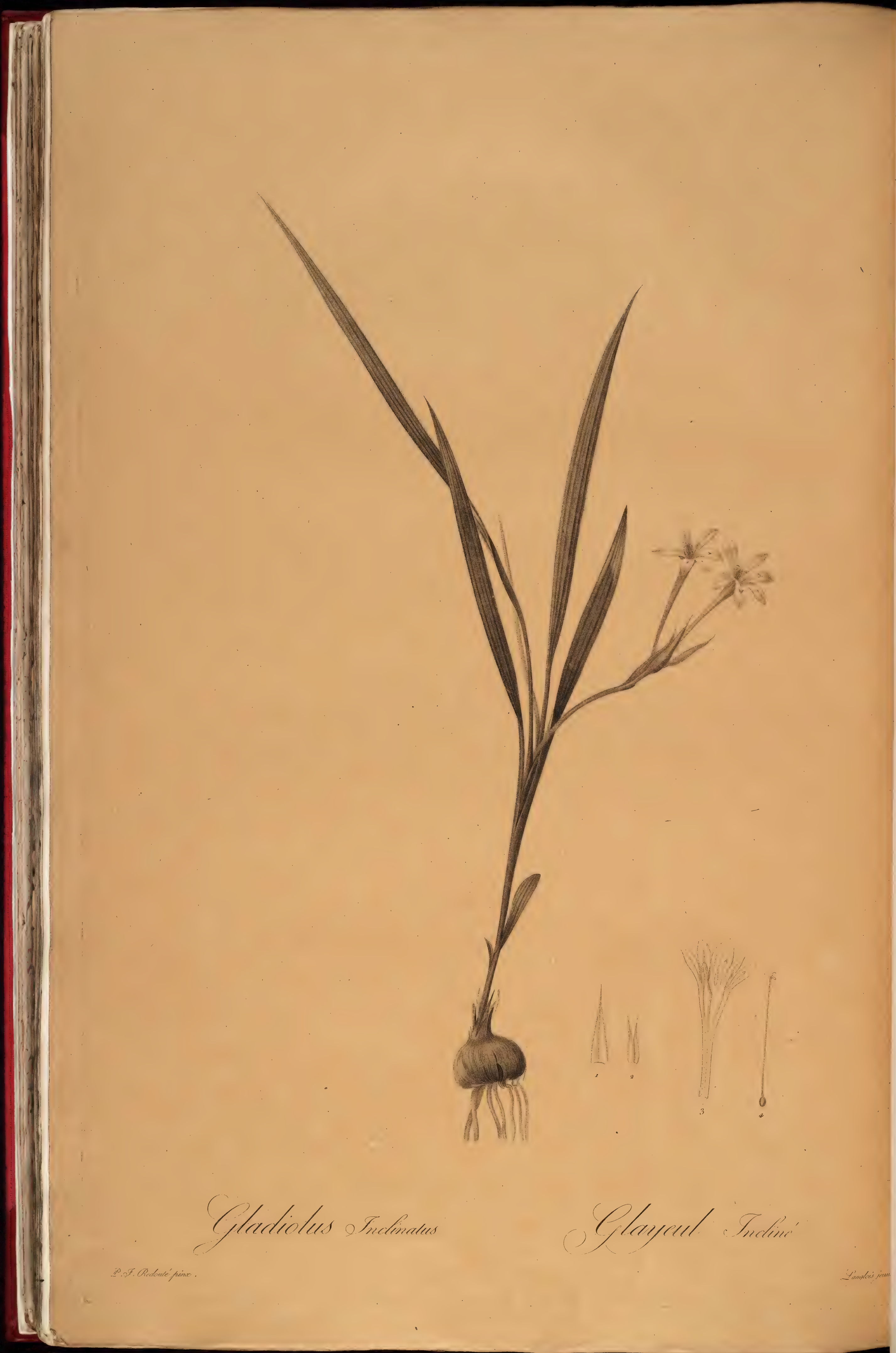




$$
\psi
$$





\title{
MOROEA IRIDIOIDES.
}

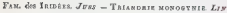

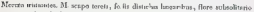

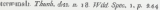

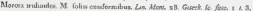
bop thor Schanbr: $=6200$

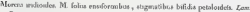

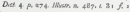

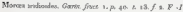

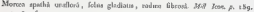
$t=8 \%, \mathrm{~h}$.

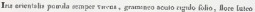

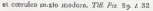

\section{MOREE FAUSSE-IRIS.}

\section{DESCRIPTIO}

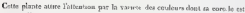

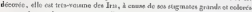

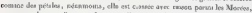

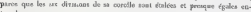
tro slat

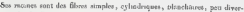

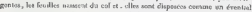

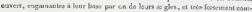

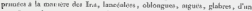

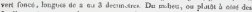

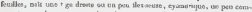

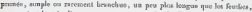

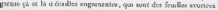

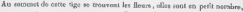

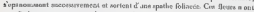

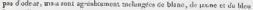

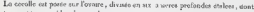

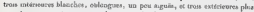

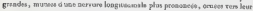




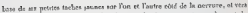

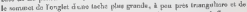

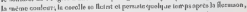

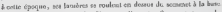

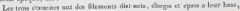

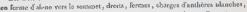

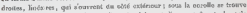

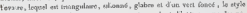

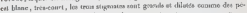

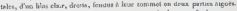

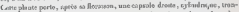

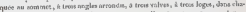

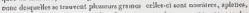

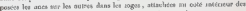

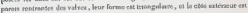

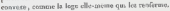

\section{II $18 \mathrm{~T}$ if $\mathrm{I}$ is $x$}

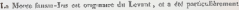

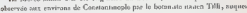

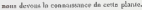

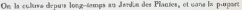

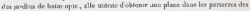

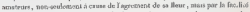

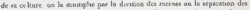

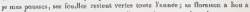

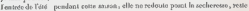

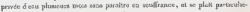

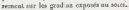

\section{BXPLICATION DE LA PLANGHE}

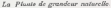

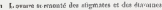

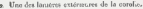

9 ha coroule anse it peticeale

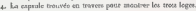

b I extraiae. 


$$
\sqrt{2}
$$




\section{ALSTROEMERIA PELEGRINA.}

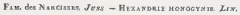

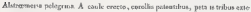

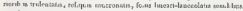

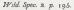

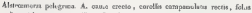

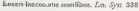

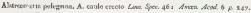

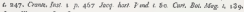

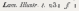

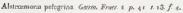

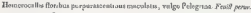
a p 71a 62.

\section{ALSTROE HERIA PELEGRIXA}

\section{DESCRIPTIOX}

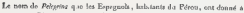

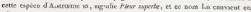

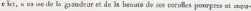
sbitaks rolpaisurwasent

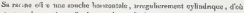

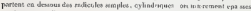

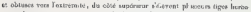

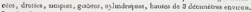

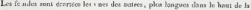

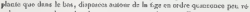

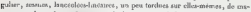

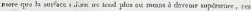

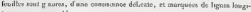
tucimech peu amsilim.

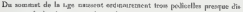

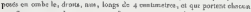

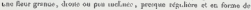

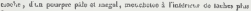
fousta 


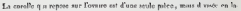

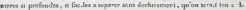

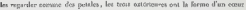

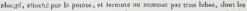

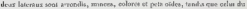

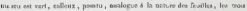

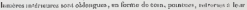

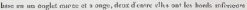

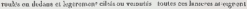
3 ceasontose de longunn

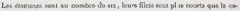

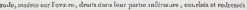

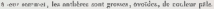

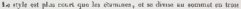

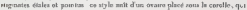

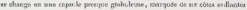

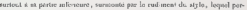

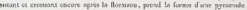

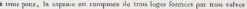

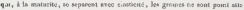

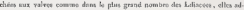

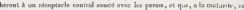

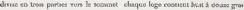

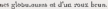

\section{1 S T O I $\mathrm{N} \mathrm{F}$}

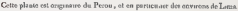

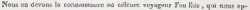

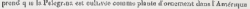

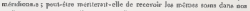

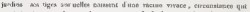

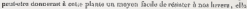

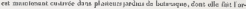

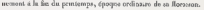

\section{EXPLICATLOA DE LA PLANCHE}

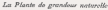

- Lo patin et he plamives bilizransis da ta cosolle

2 Ler rocel 


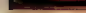




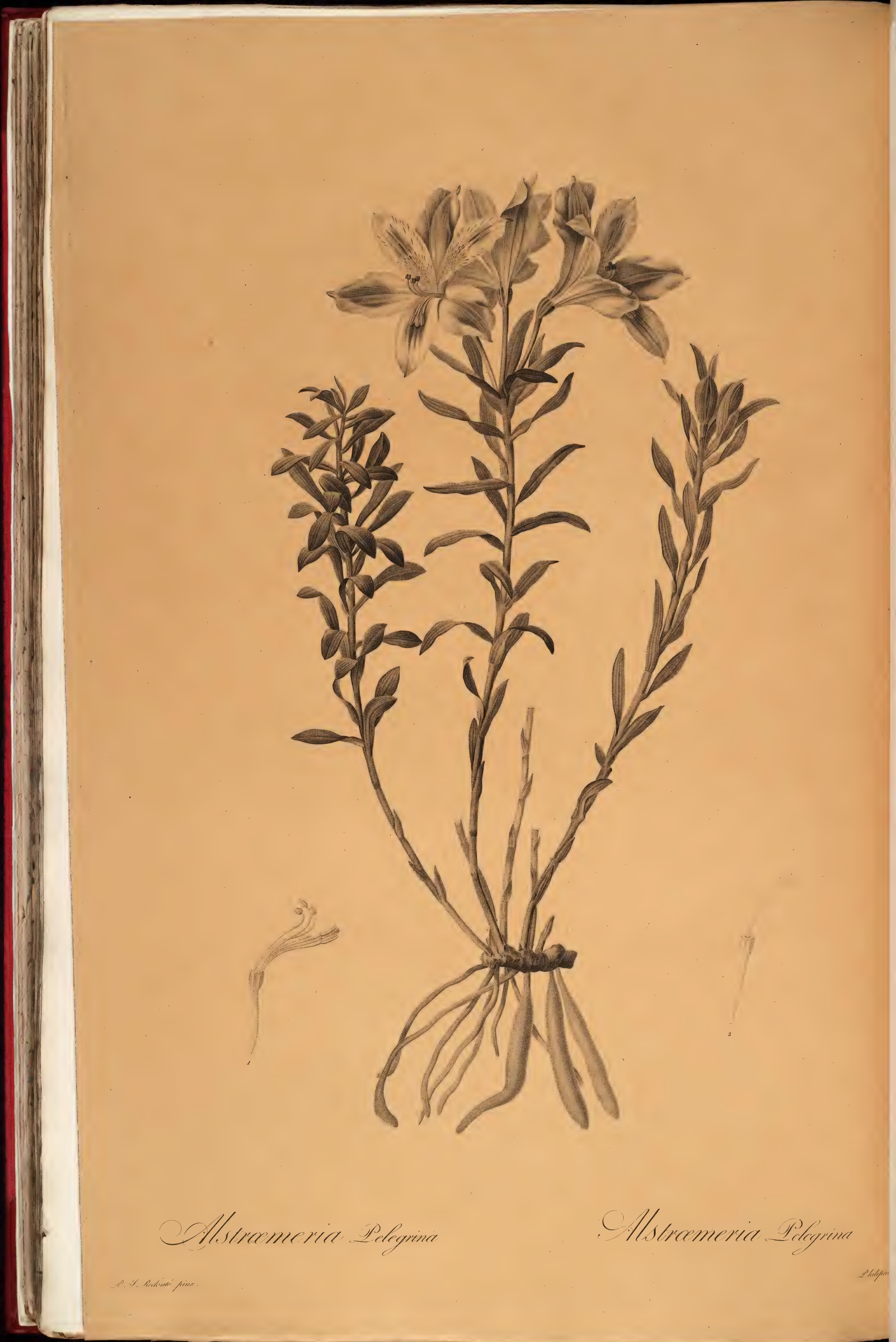




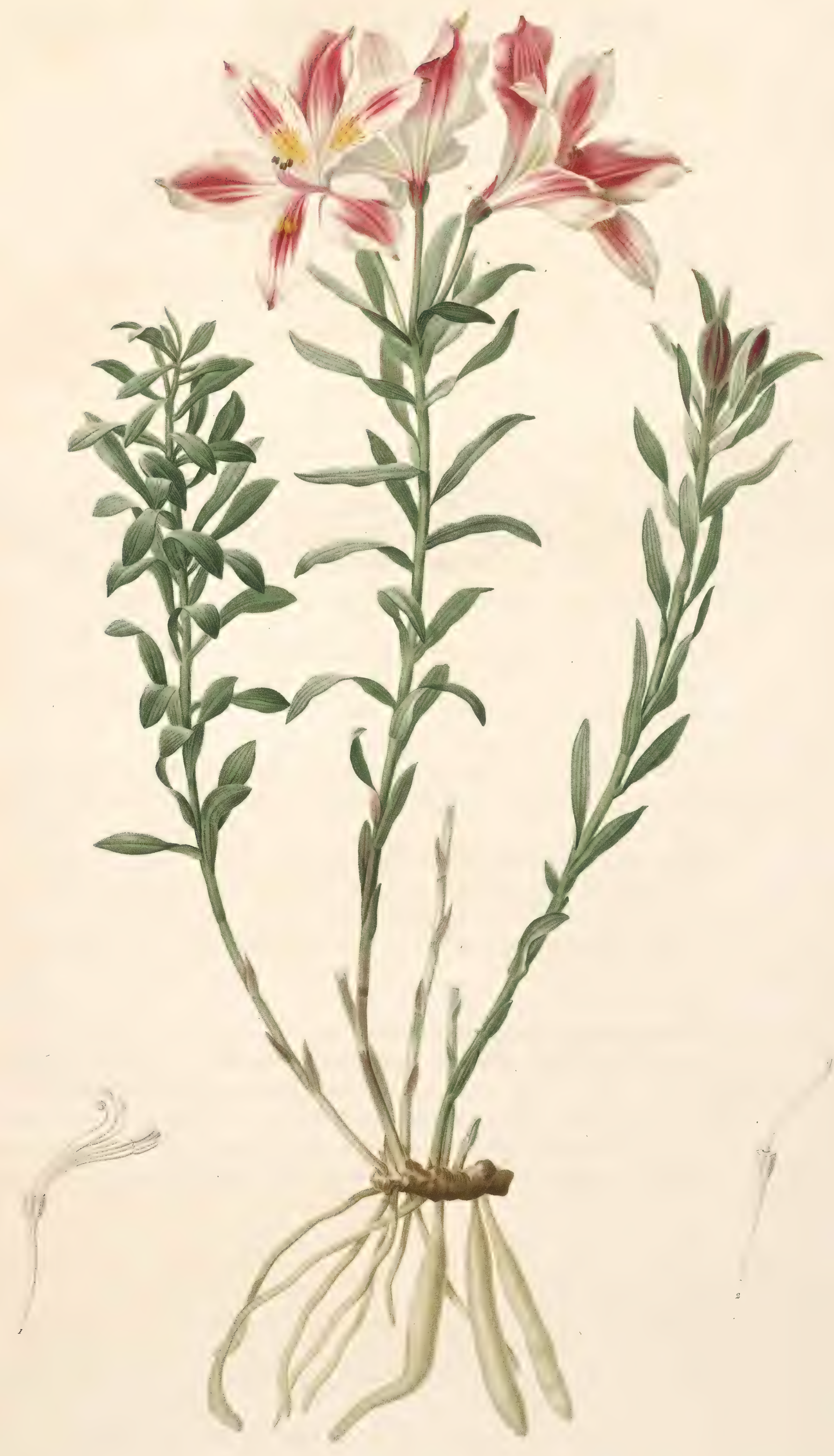

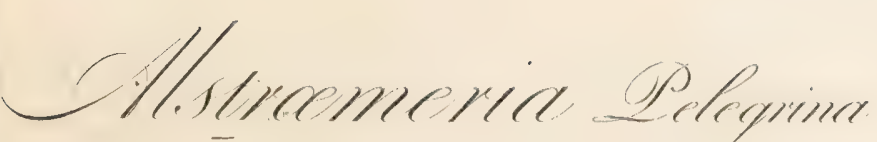

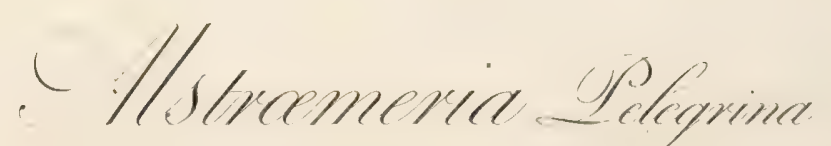




\title{
SISYRINCHIUM CONTOLUTUW.
}

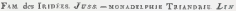

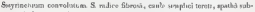

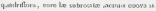

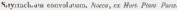

\section{BE. R ULDIENNE ROULER}

\section{DISCRIFTION}

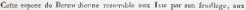

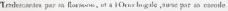

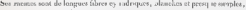

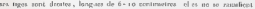

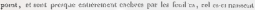

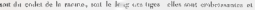

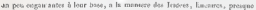

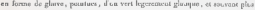
angote que la fiǵ

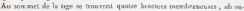

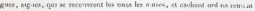

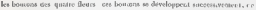

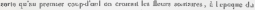

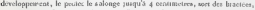

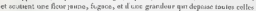
Ae a sures

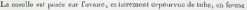

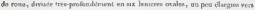

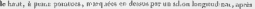

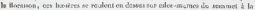

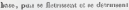

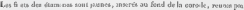

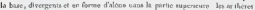

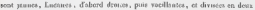

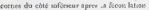

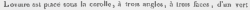

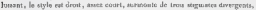

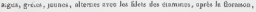




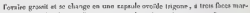

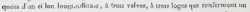

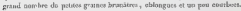

\section{It 1 \& T O I I}

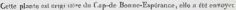

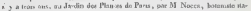

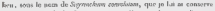

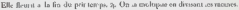

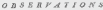

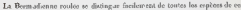

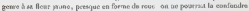

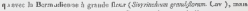

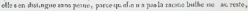

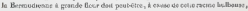
Ptre raprotion su ganne \&alinan

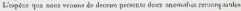

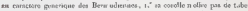

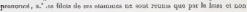
tasa foulo drur las gincur.

\section{BXPLICATIONDE LA PLANCBE}

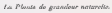

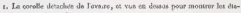

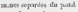

2. Le prist

3. La parenes.

4. Quelioes grance. 


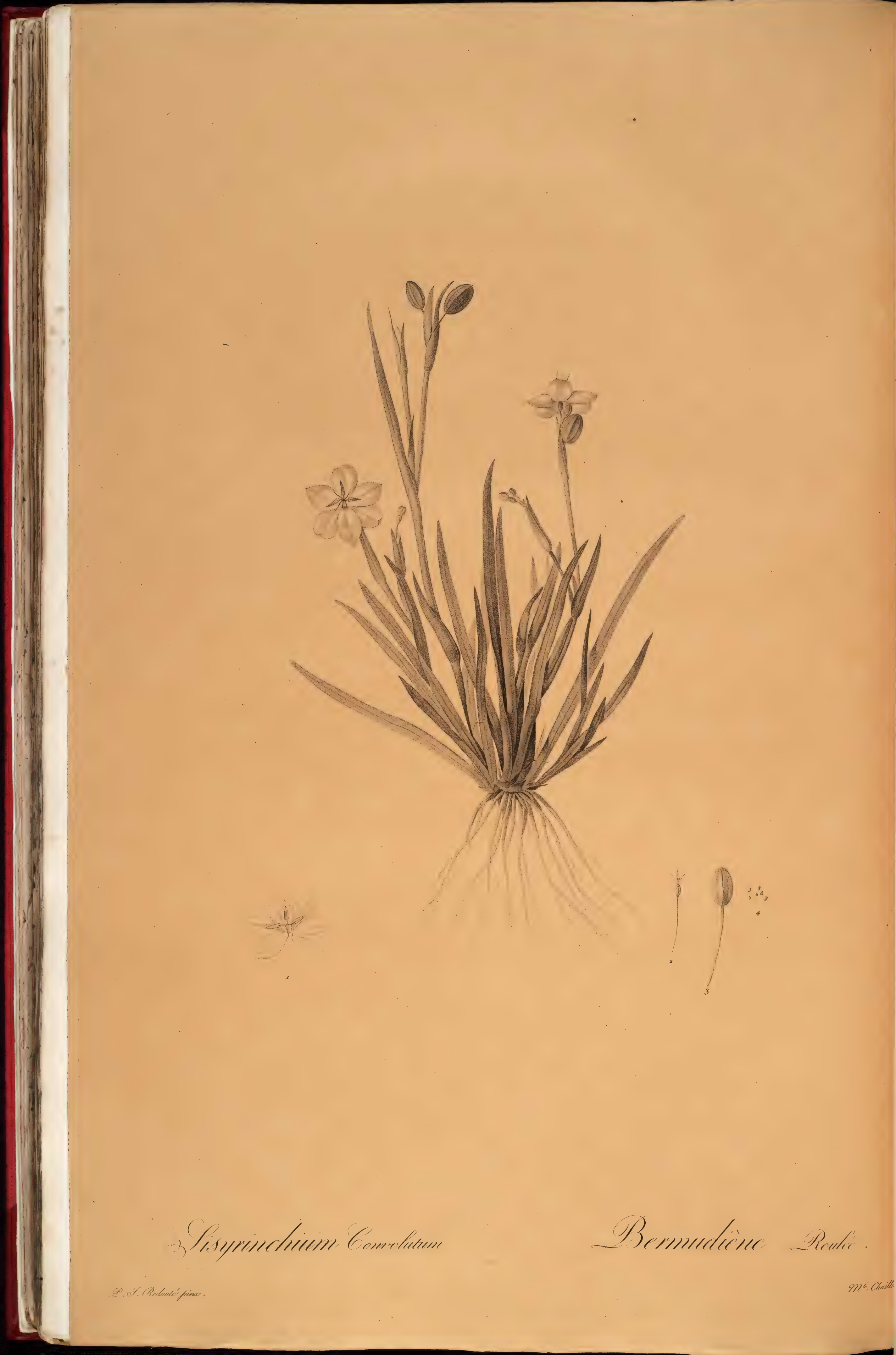





\title{
IRIS TOBEIOSA.
}

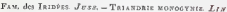

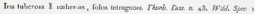
p. 540

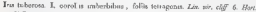

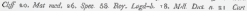
$B \times 5$ ato 4,53

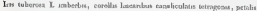

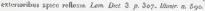

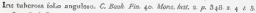

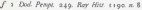

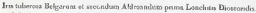
$\operatorname{Lot} k \mathrm{~kat}, \mathrm{o}^{\mathrm{s}}$

Heriadisctylue bolse guadrangale, Tasen, Gor: bo

Tace Heconadacte Yals

\section{IIS TUBEAEUSE}

\section{B C R IP $T$ I 0}

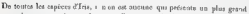

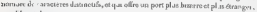
Fue Ina taterecise

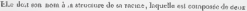

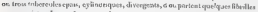

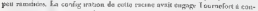

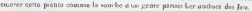

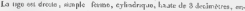

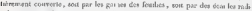

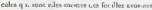

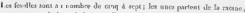

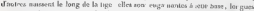

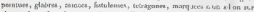

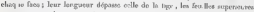

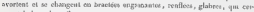
g. neot la bene do a Deric 


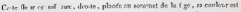

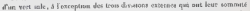

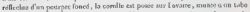

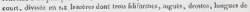

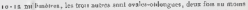

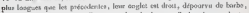

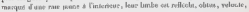
pi d an poui pre nomule

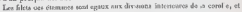

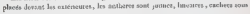
ves rigeinzets.

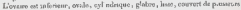

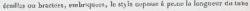

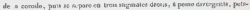

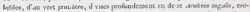

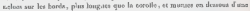

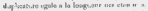

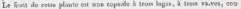

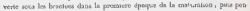
What J a $n$ aini..

\section{1 - 10.15 .8}

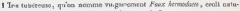

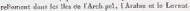

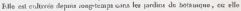

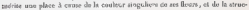

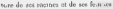

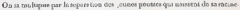

\section{EX YLICATION D\& LA P L A C R R}

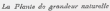

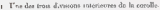

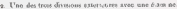

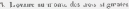






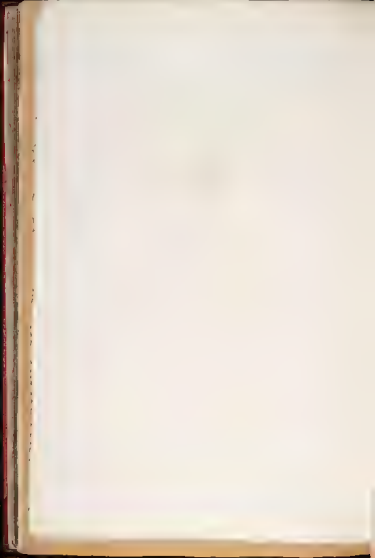




\section{KOEMPFERIA LONGA.}

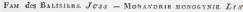

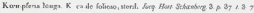

\section{RE IPETRIR IOXG U.}

\section{DESCA E F T I O N}

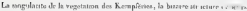

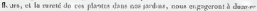

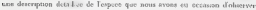

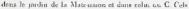

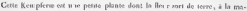

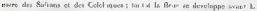

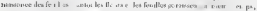

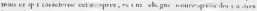

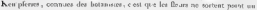

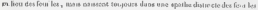
enurane daes lyo Aryan lics

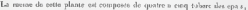

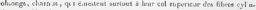

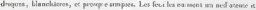

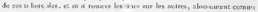

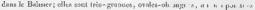

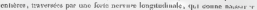

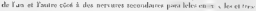

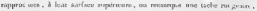

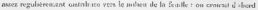

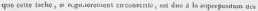

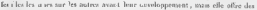

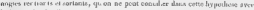

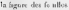

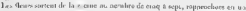

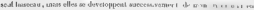

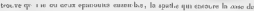

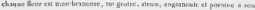

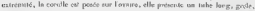

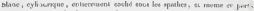

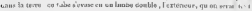




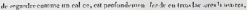

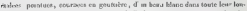

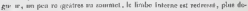

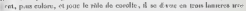

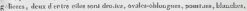

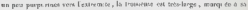

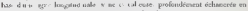

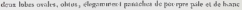

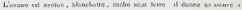

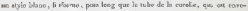

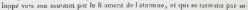

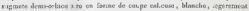
c licesur les terils

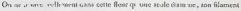

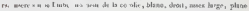

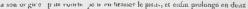

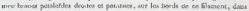

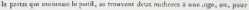

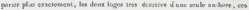

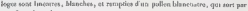
me finte Linerale

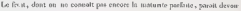
elte, late sepale a troa loges et at trou va ves

\section{I T T I I E.}

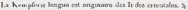

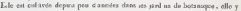

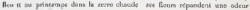
almet of vesten the

\section{LKRLICATIOC DE LA PIAXCAF.}

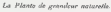

1 Dre llewiandre.

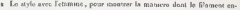
leare 1 p pail

8. Lu firith.

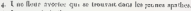

d. Le praic de la teur arsation. 


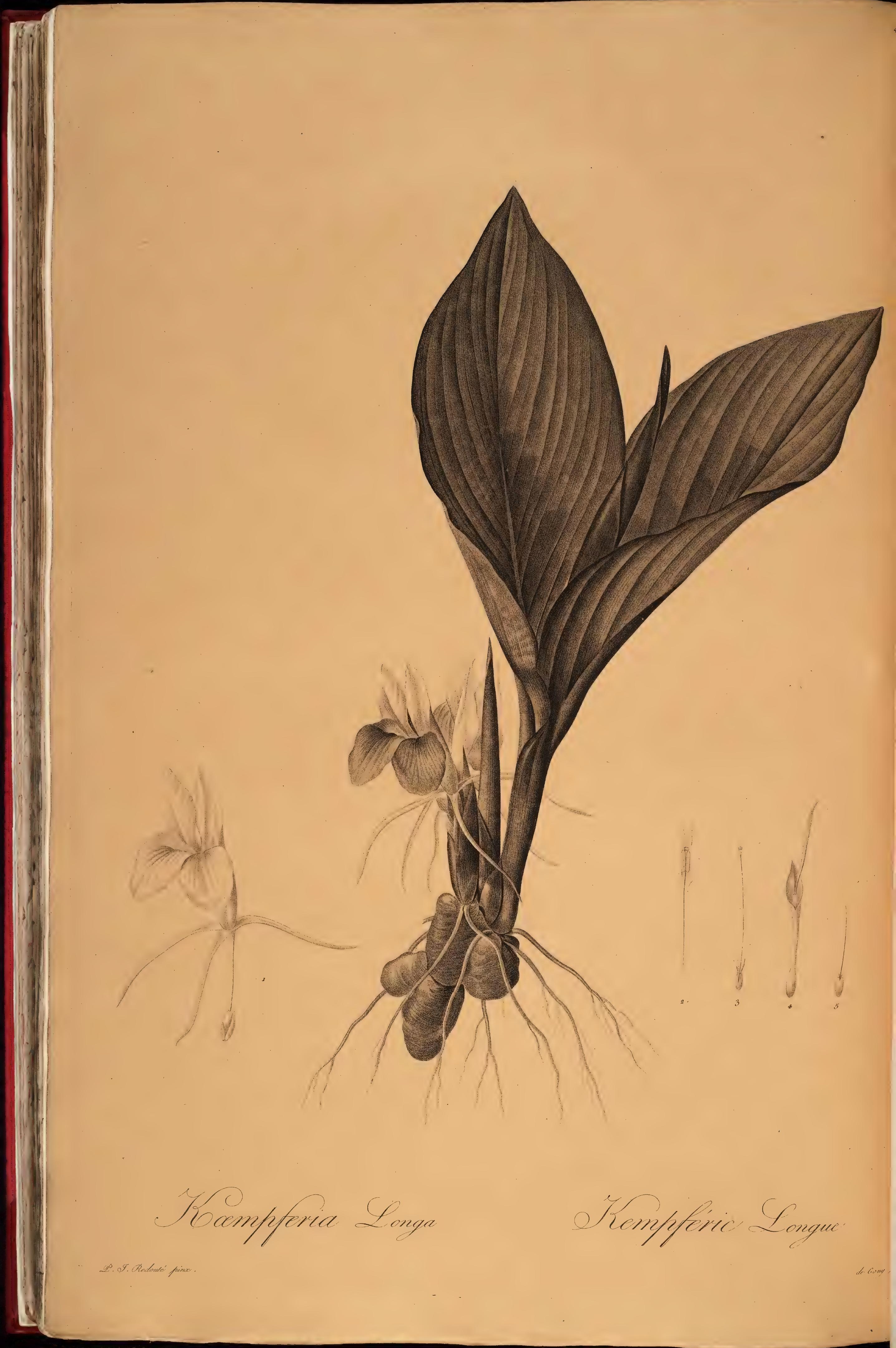




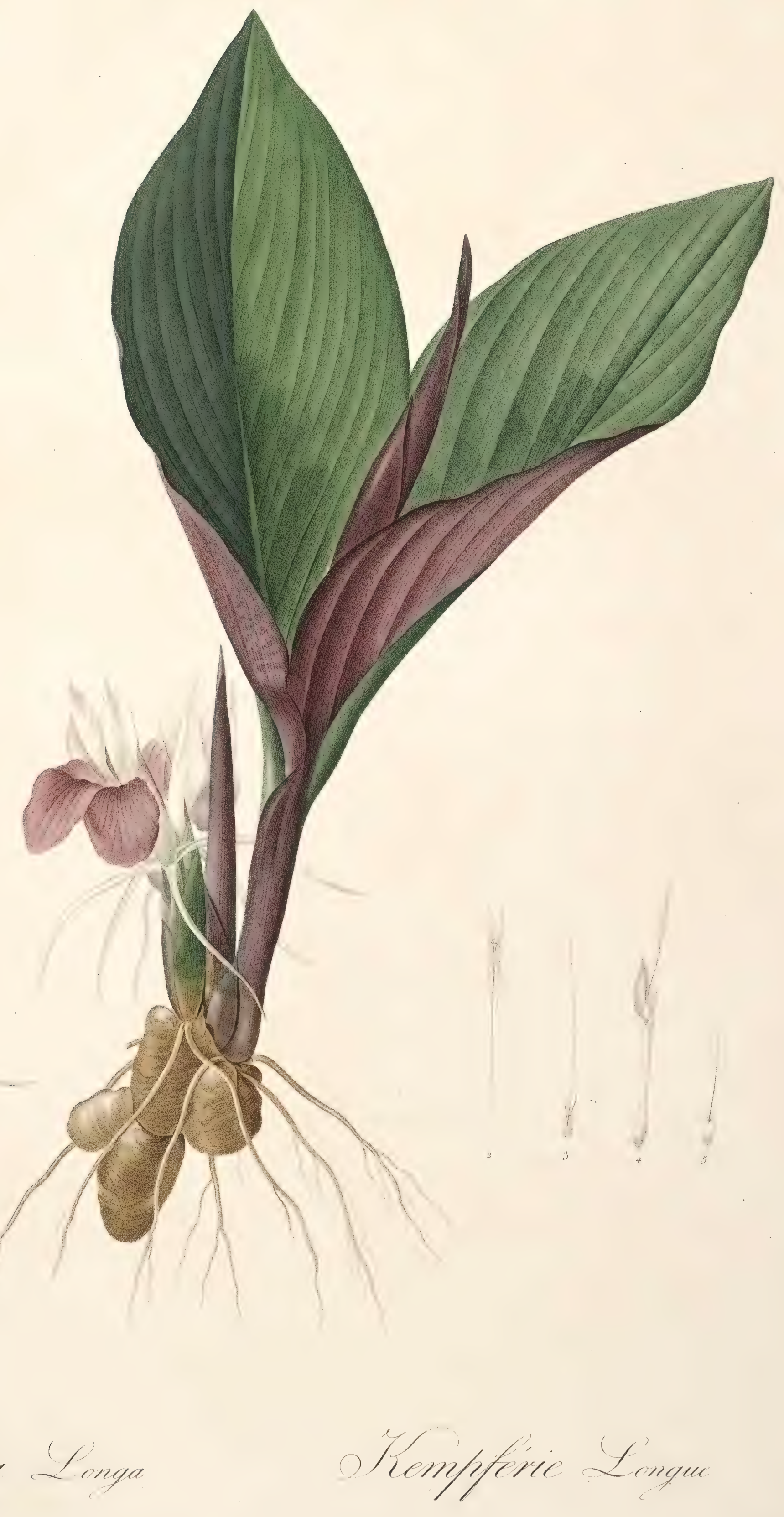

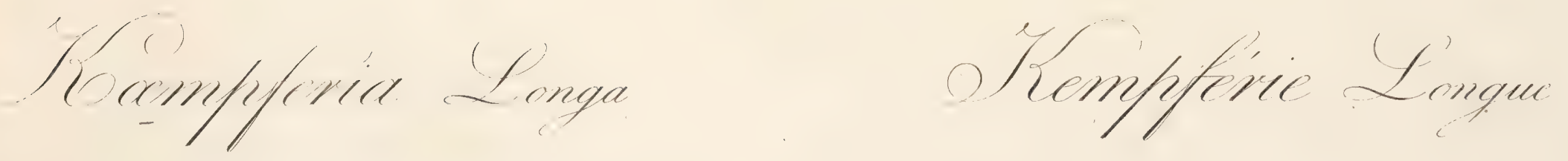




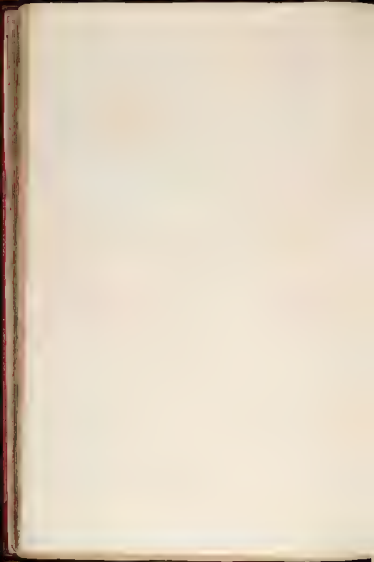




\section{A L L IU M STR IATEM.}

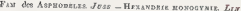

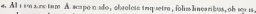

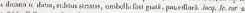

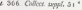

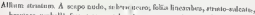

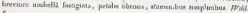
Pex \& \& 79

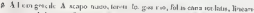

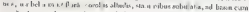

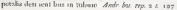

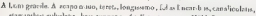

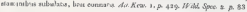

\section{AIL STRIE}

\section{DESCRTPTION}

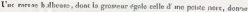

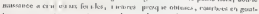

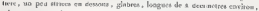

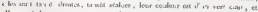

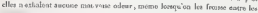
II 1 is.

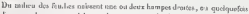

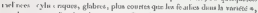

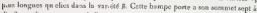

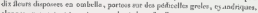

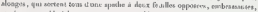

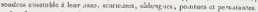

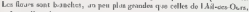

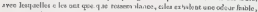

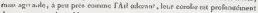

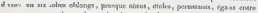

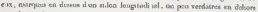
prìa da wave lavo

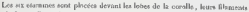

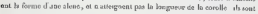




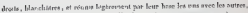

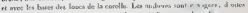

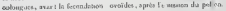

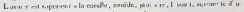

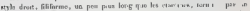

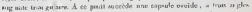

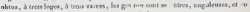

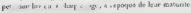

\section{15. T D I I I}

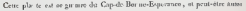
te la Ja nasiot 1

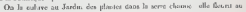
prentems

\section{OBSARARTONS.}

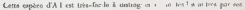

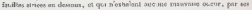

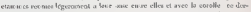

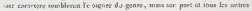

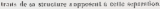

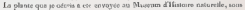

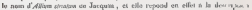

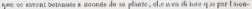

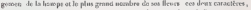

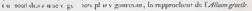

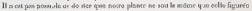

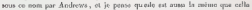

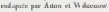

\section{EXPLICATION UE LA PLA R C B L}

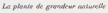

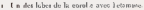

- De pasir

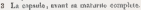

4 Ls ospari of arrevera.

\$, Quelyaet kres net 


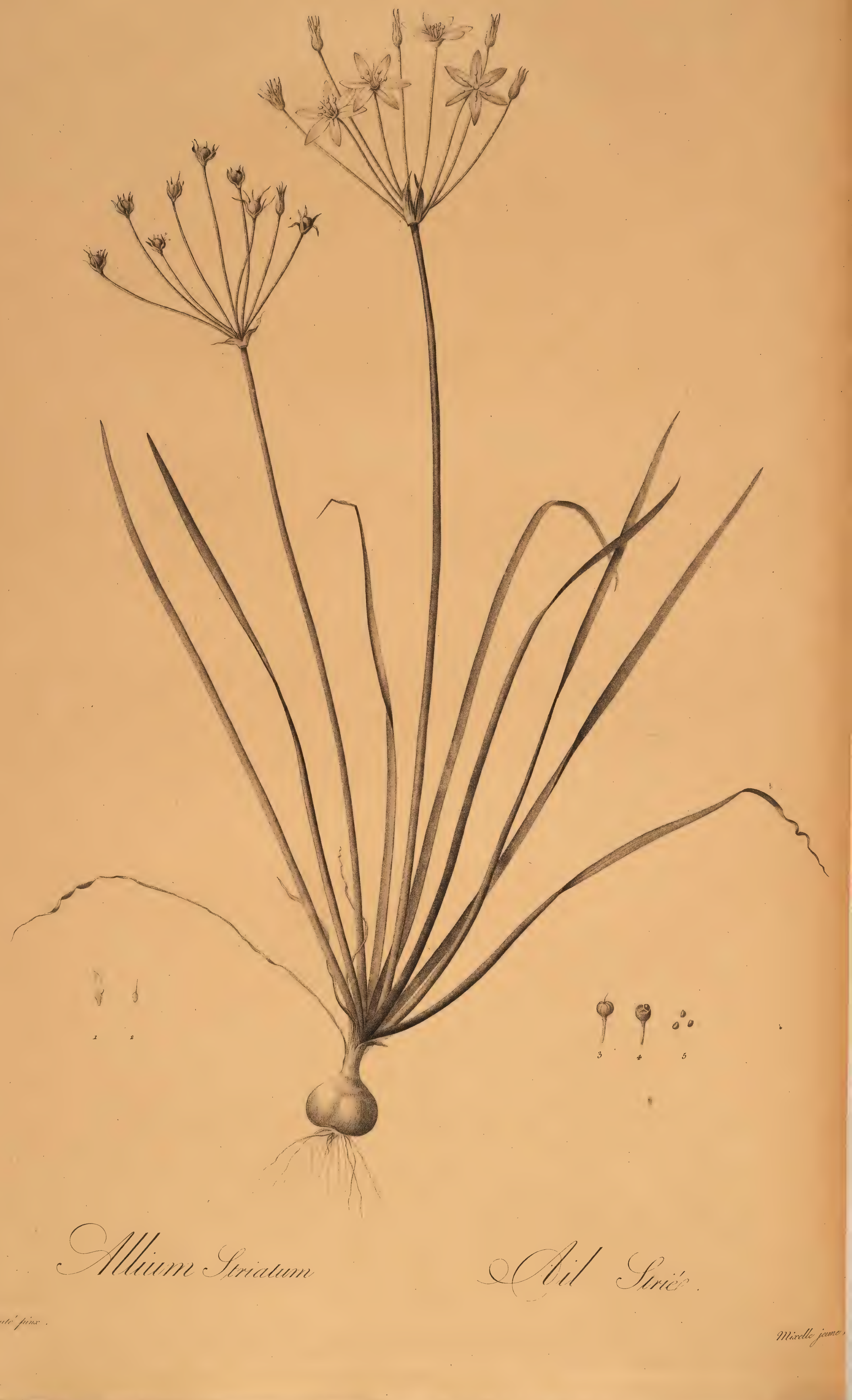




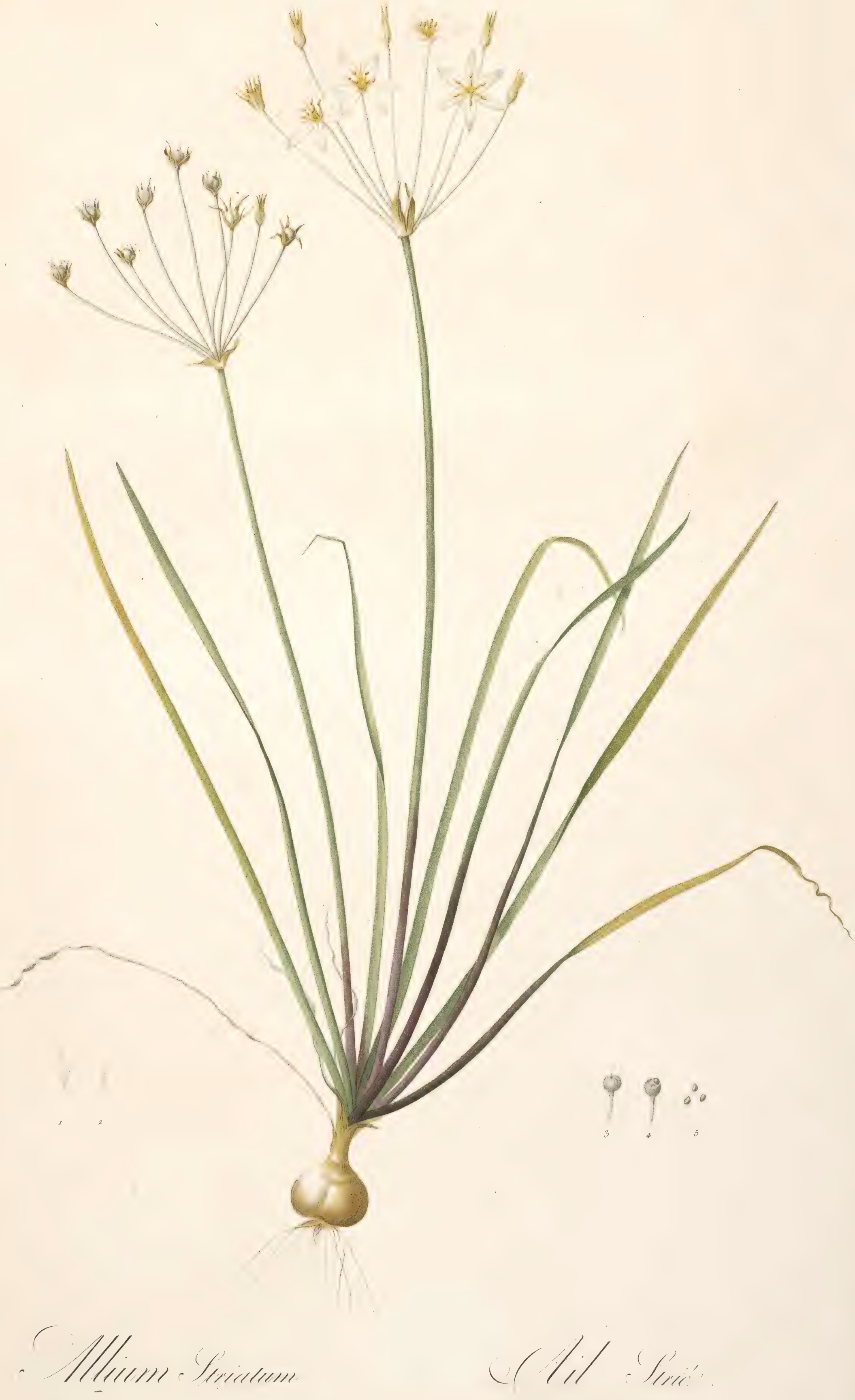




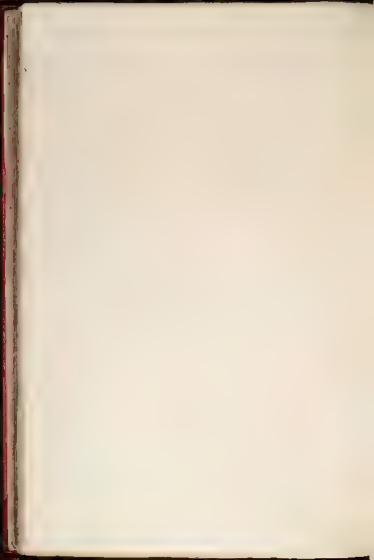




\title{
FRITILLARIA LATIFOLIA.
}

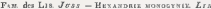

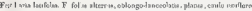

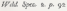

\section{FRITILLAIKE A LATGB FEUILIT}

\section{DISC $\mathrm{AI}$ II0}

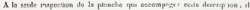

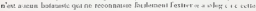

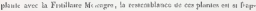

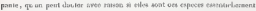

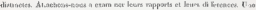

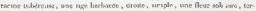

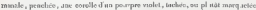

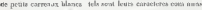

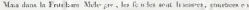

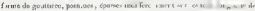

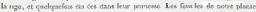

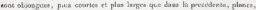

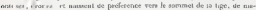

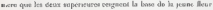

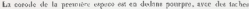

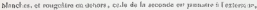

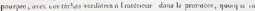

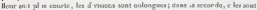

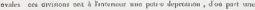

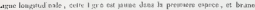
dose ho sarve it

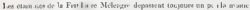

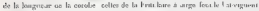
n ne:

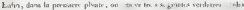

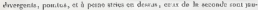

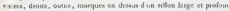




\section{I)}

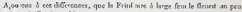

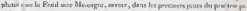

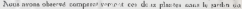

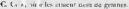

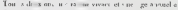

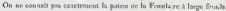

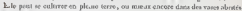
pe iding it wer

$$
\text { OASEAPATIDNS }
$$

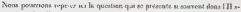

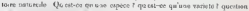

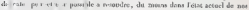

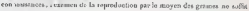

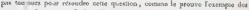

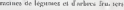

\section{EXPEICATIOY DE LA P L N T C E E}

\section{Le Planls de enandieve maturnille}

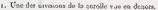

- Ia muna nor en delina

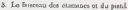

4. La parat. 



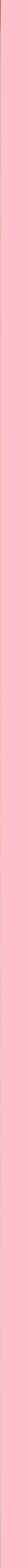




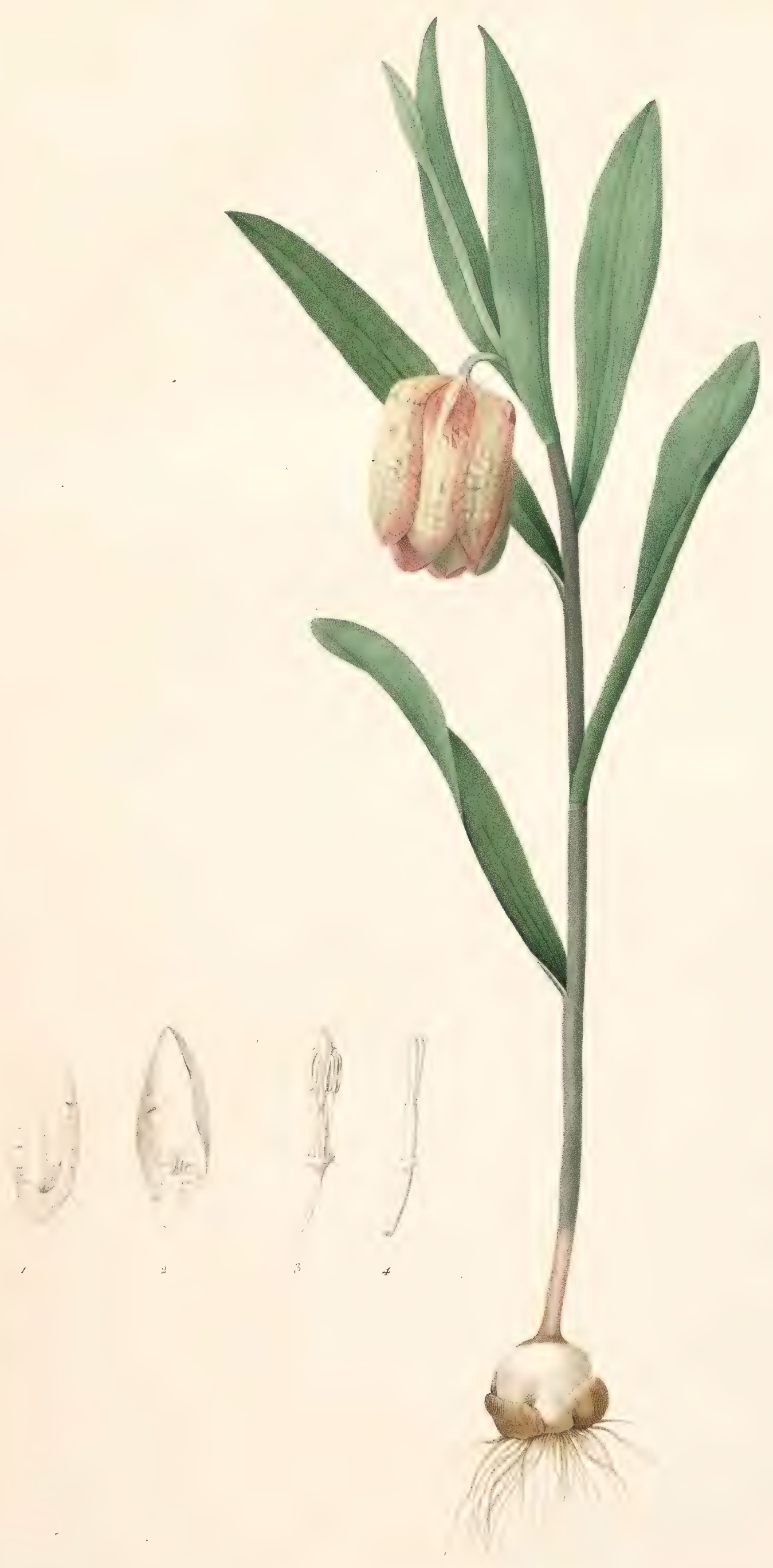

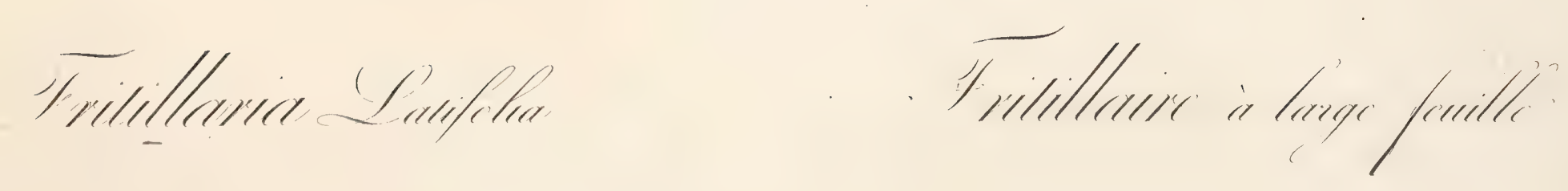


P 


\section{LACIINALIA PENDULA.}

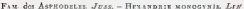

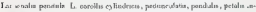

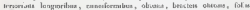

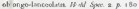

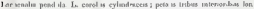

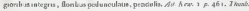
ind of of

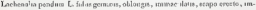

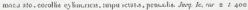
colis 5 sat?

\section{LACHENALE PHNANTE}

\section{TBSCDTPTIOX}

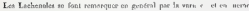

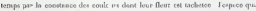

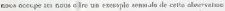

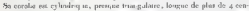

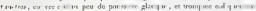

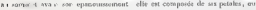

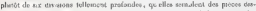

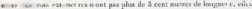

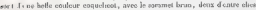

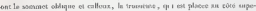

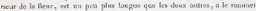

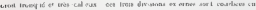

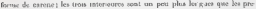

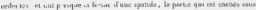

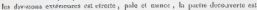

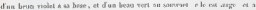

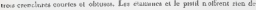
partaculier bे conte enpsot.

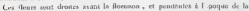

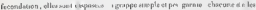

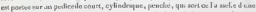

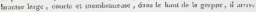




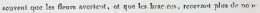

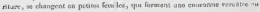

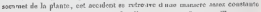

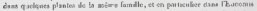

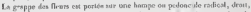

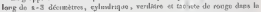

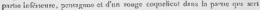

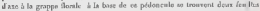

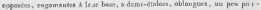

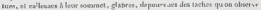

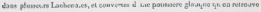
suc a hamper

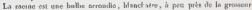

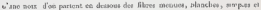
oylindrogoce.

\section{BISTOI I E}

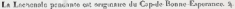

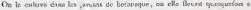

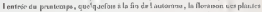

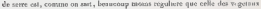
culvere en ples th

\section{OASERPATHQNS}

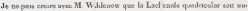

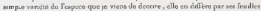

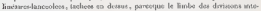

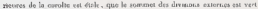

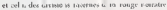

\section{EXPLICATION DE LA PLANGUE}

Lo Phuire de fraudion wafornile

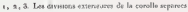

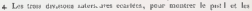
sit $\times$ iriese

I L prote 

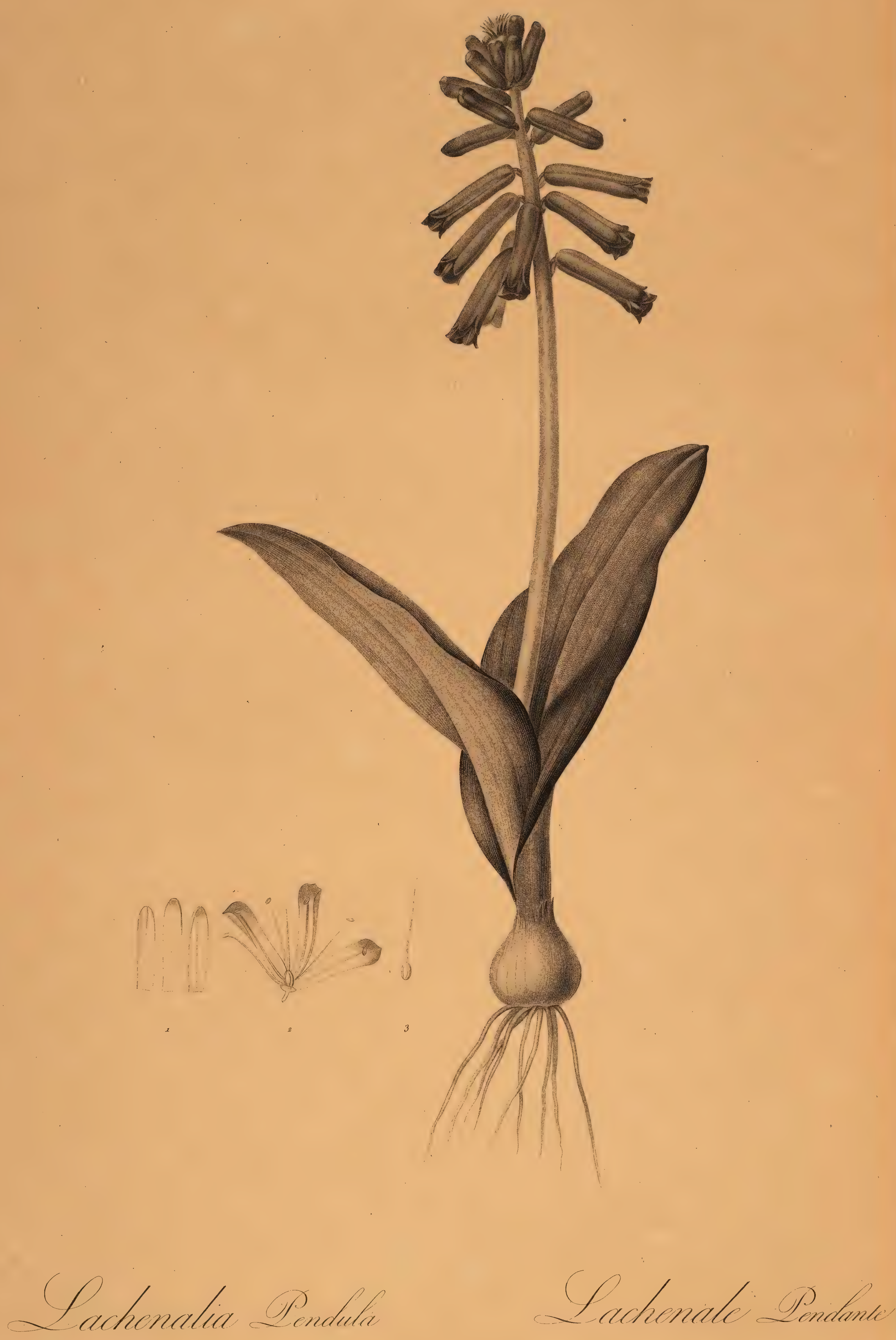


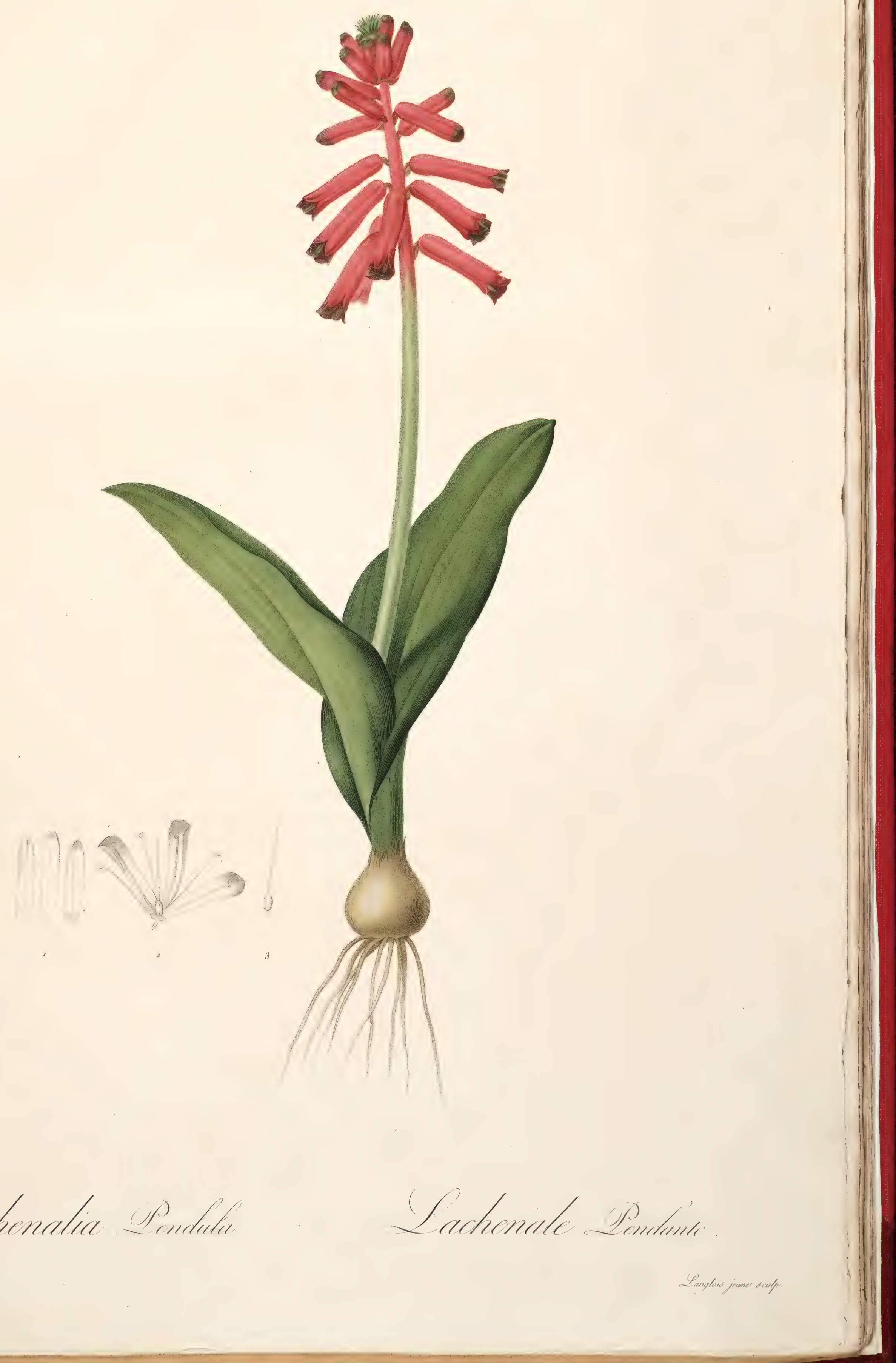




\title{
MONTBRETIA.
}

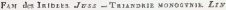

\section{CIARAOTRE GENEREUS}

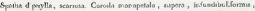

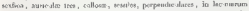

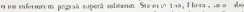

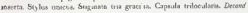

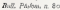

\section{ง 0 N T B I I T T I F}

\section{OASA ANATHOAs}

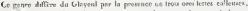

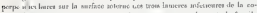

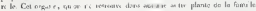

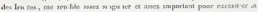

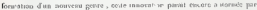

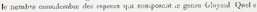

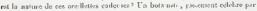

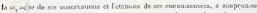

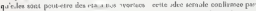

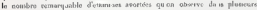

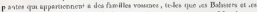

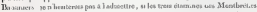

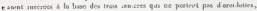

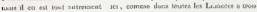

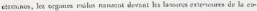

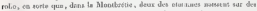

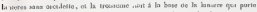

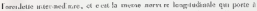

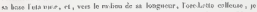

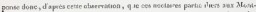

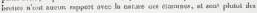
pretuberamen ba la eseotlo 


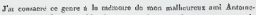

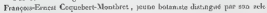

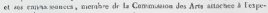

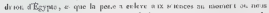

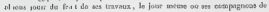

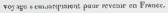




\section{HONTBRETJA SEGURIGERA.}

Ghitol as teournger Covt boe sug 4.383

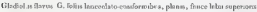

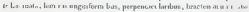

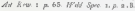

\section{WON FBRETE PORTE. ITCHE.}

\section{5 C. I I F T O X}

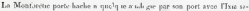

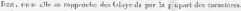

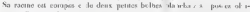

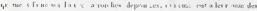

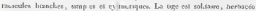

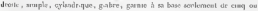

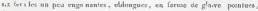

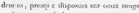

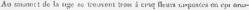

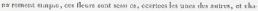

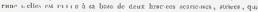

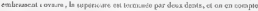

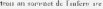

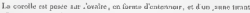

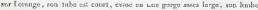

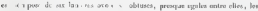

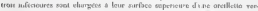

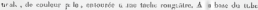

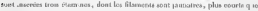

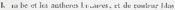

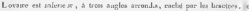

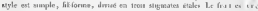

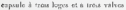

\section{1 T I 조}

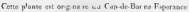

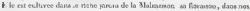

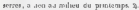




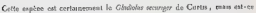

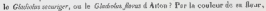

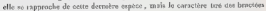

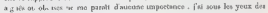

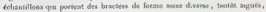

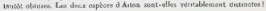

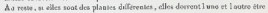
mpporides nn grean MCoutbesiet

\section{RXPLICATION DE I. T E L A C B E}

\section{La planle de grandoue nafurcilir}

I. Le oncule osveste

s. Le pisiol. 


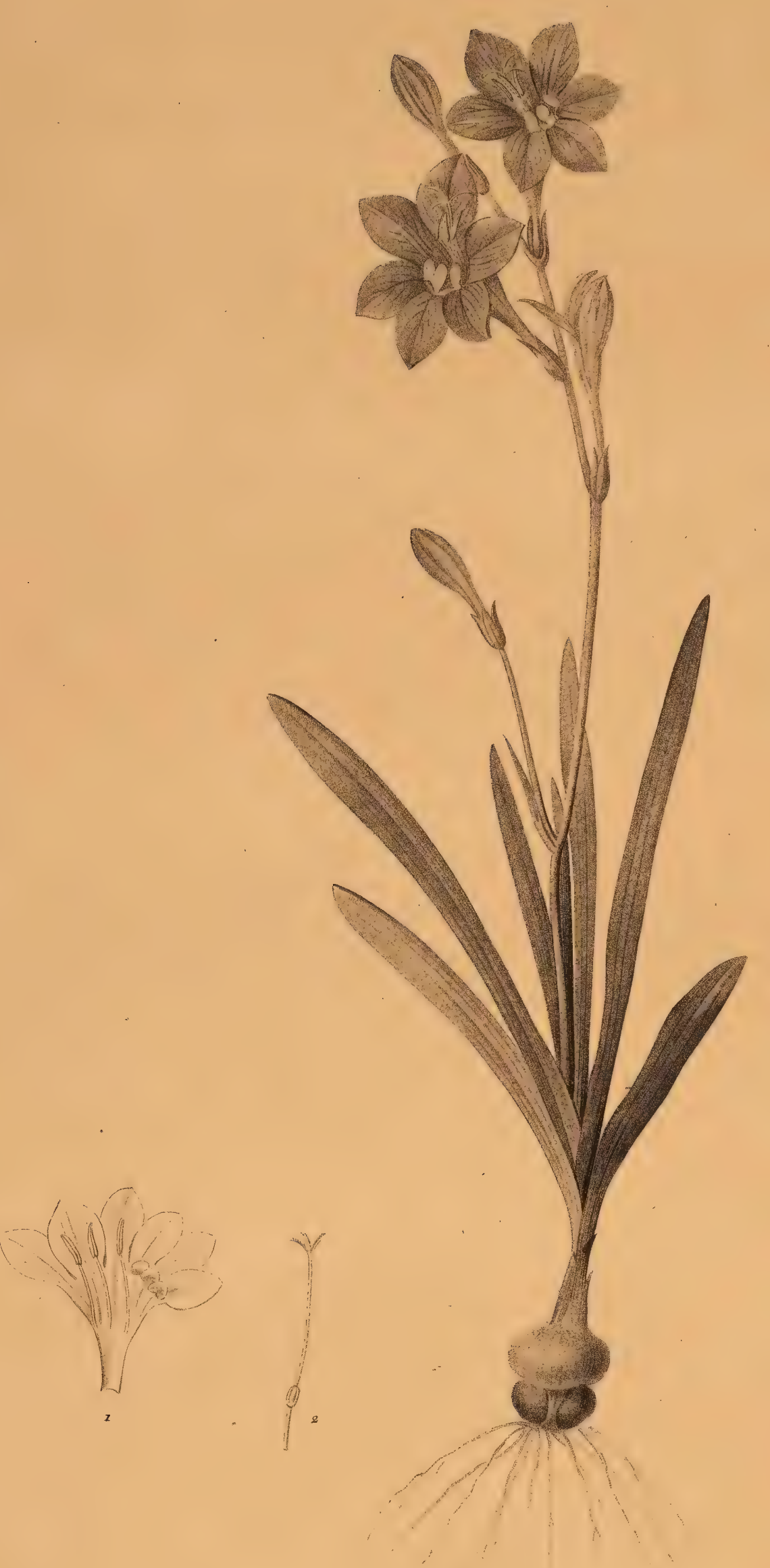

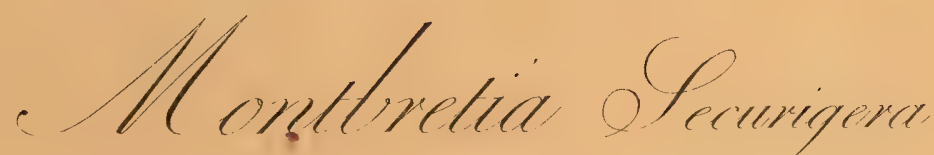

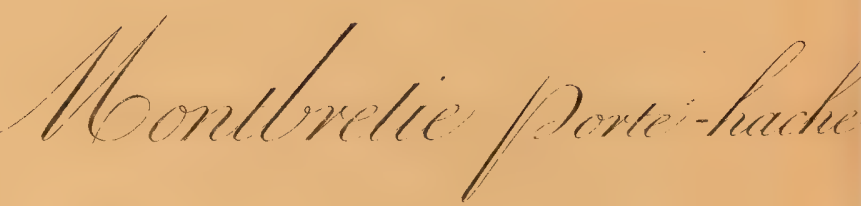




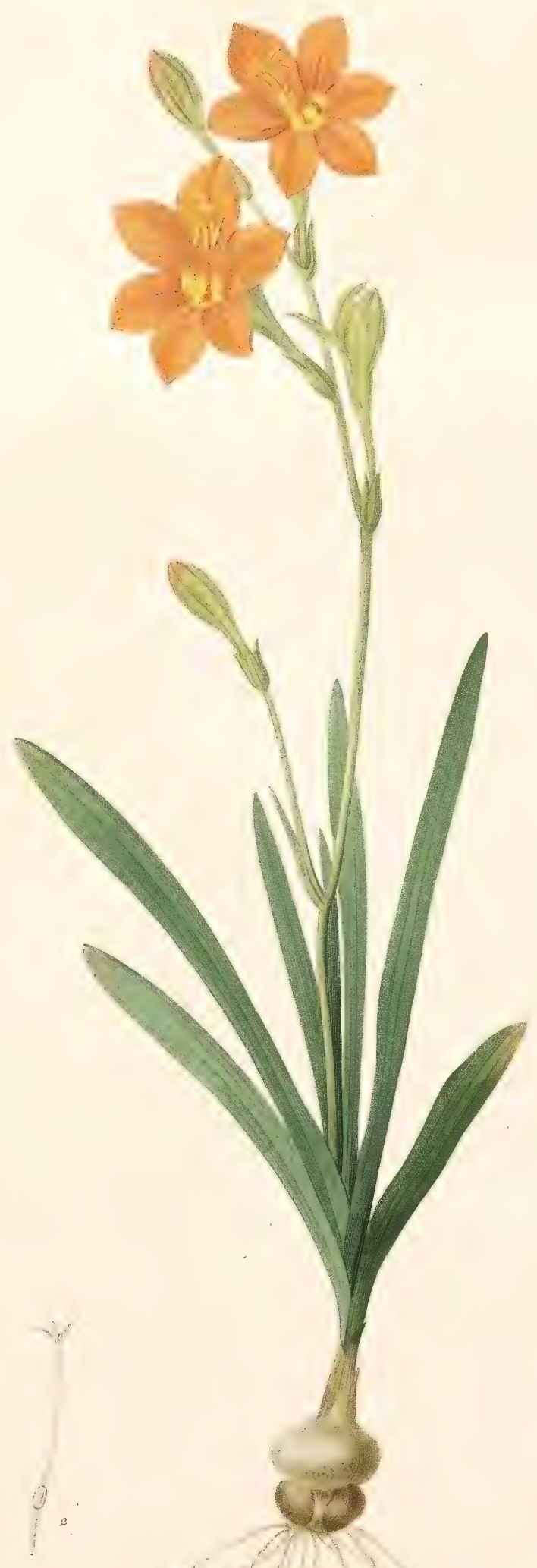

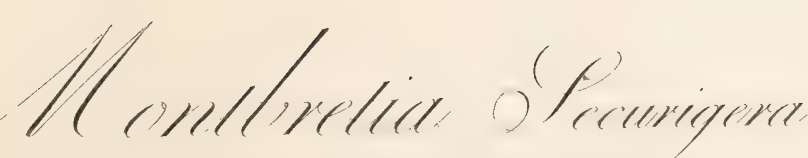

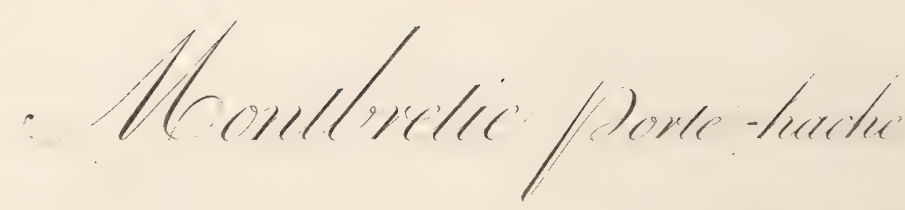




\title{
DIASIA.
}

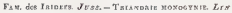

\author{
CIARAGTBR GEV
}

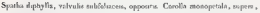

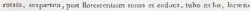

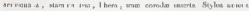

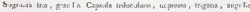

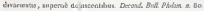

\section{1) I A S L L}

\section{OASRAPTIOYS}

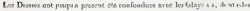

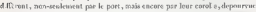

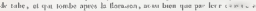

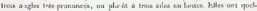

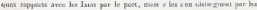

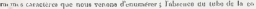

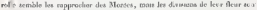

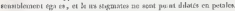

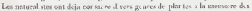

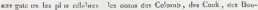

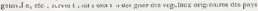

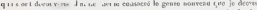

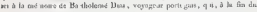

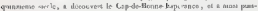

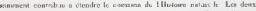

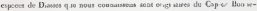
L.ppannee. 


\section{DIASTA IRIDIFOLTA.}

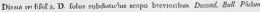
a. 8 .

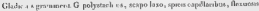

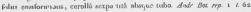
anch in

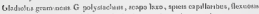

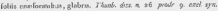

\section{DIASIE \& FUILLES D'IRIS}

\section{DESC R 1 P T I.ON}

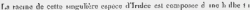

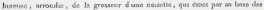

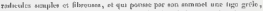

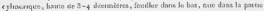

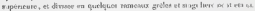

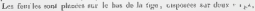

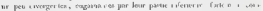

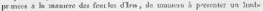

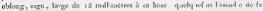

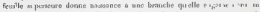

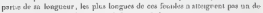

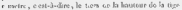

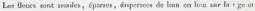

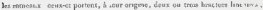

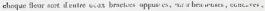

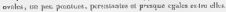

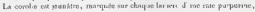

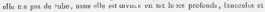

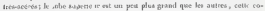

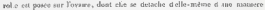

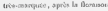

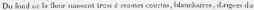

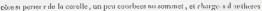

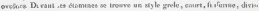

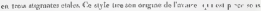

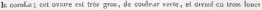




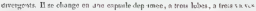

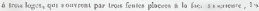

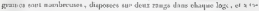

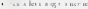

\section{I T 0 : R F}

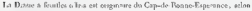

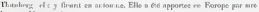

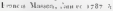

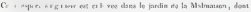

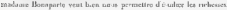

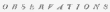

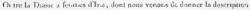

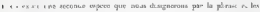
ponargates wasoais

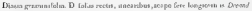
fine prowe a 8

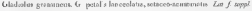

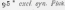

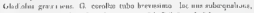

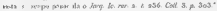

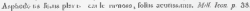
\& $6 \%$

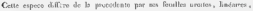

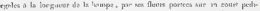

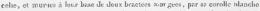

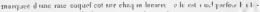

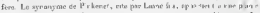

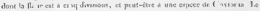

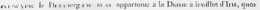

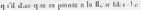




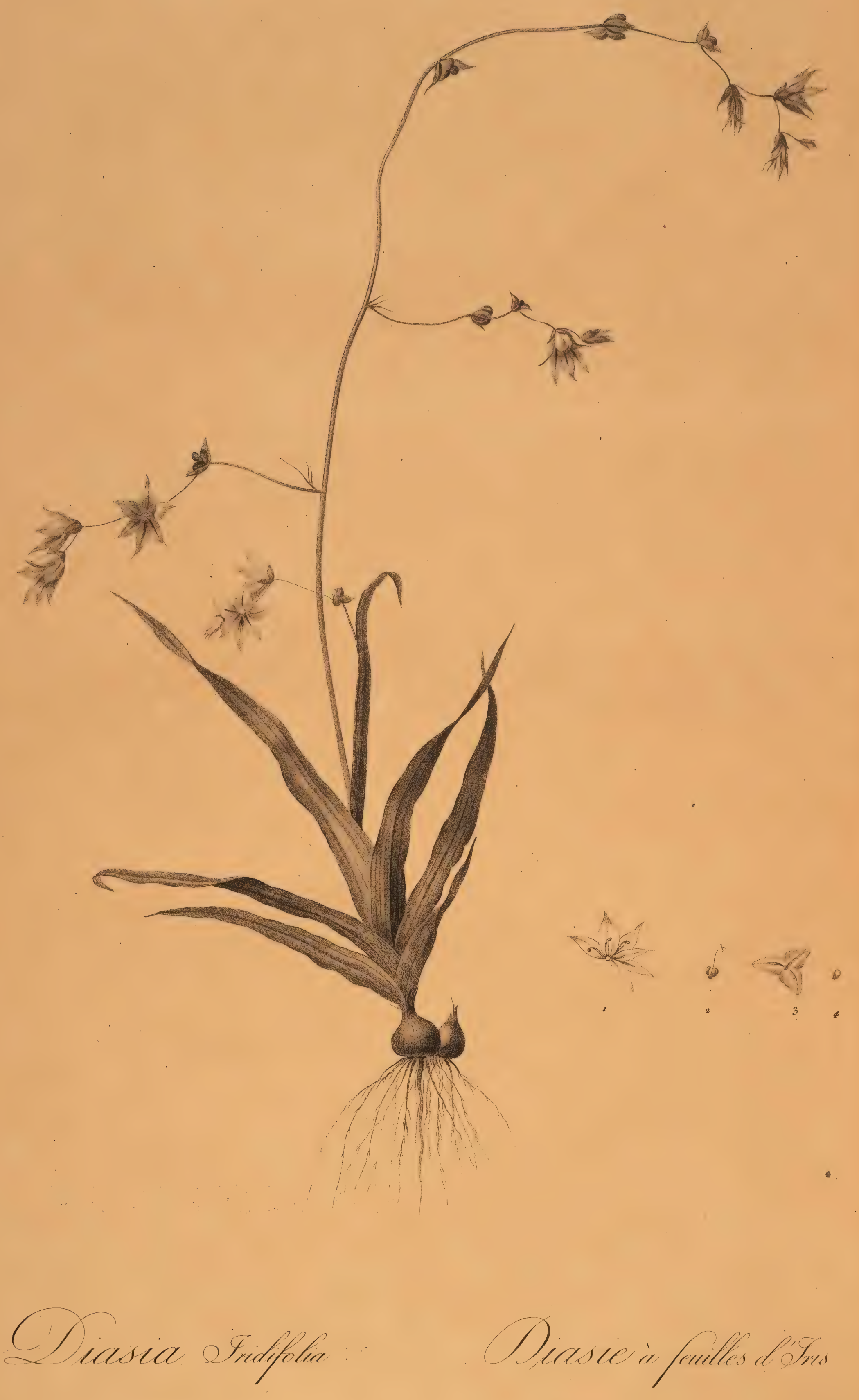




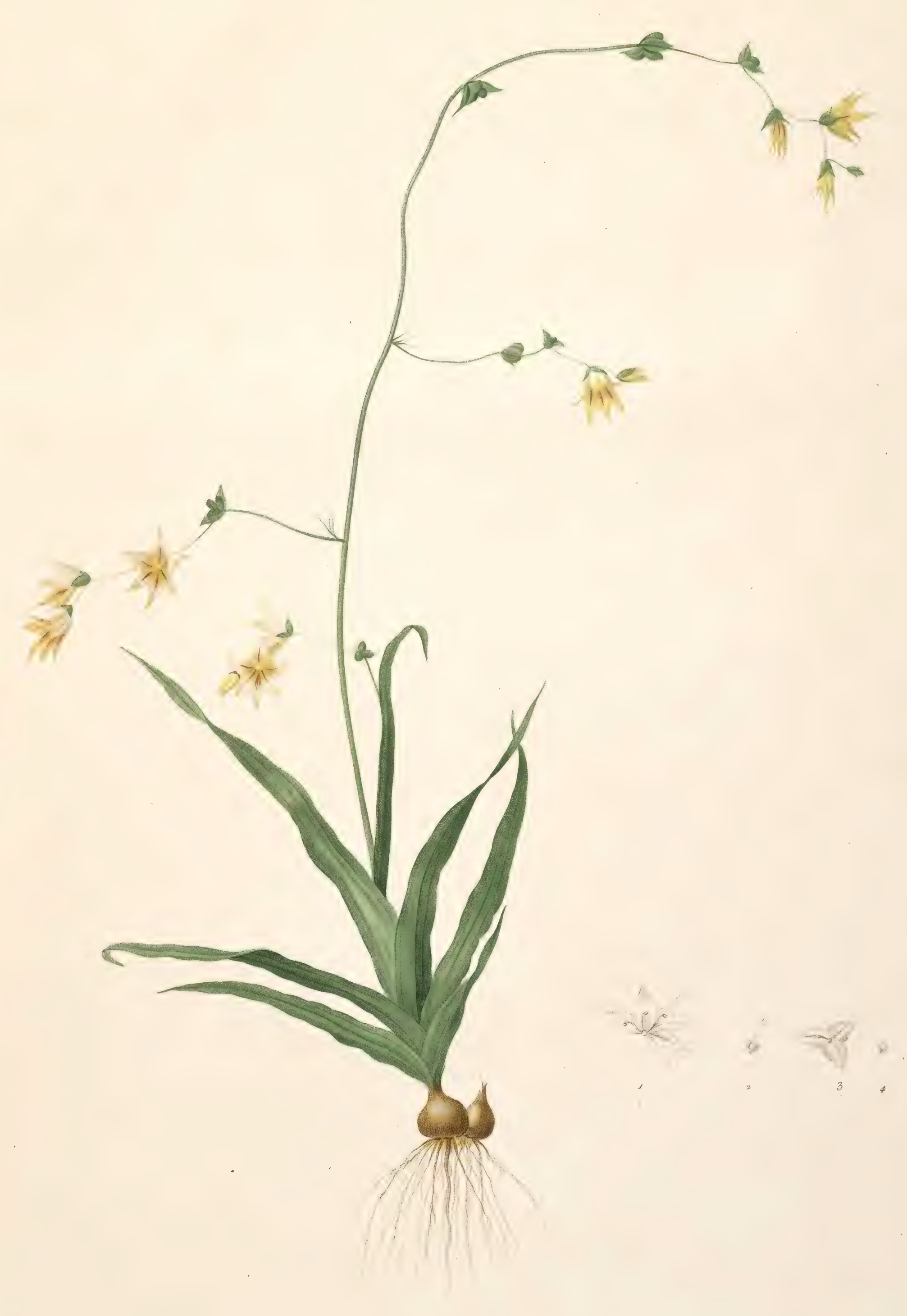

Clicesia Dymefolum

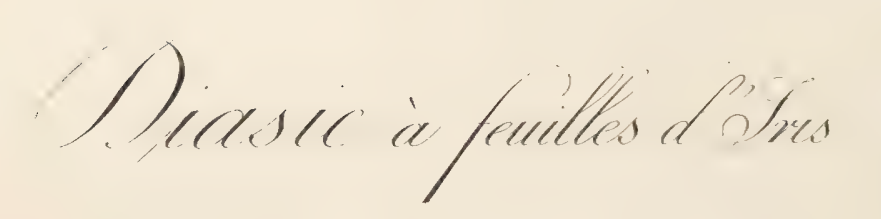




\title{
GLADIOLUS LINEATUS.
}

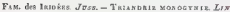

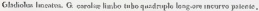

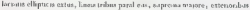

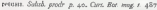

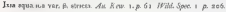

\section{GIXEUL BAYK}

\section{DFSCTIPTION}

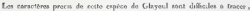

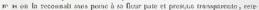

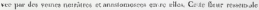

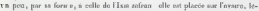

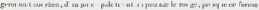

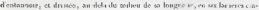

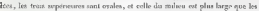

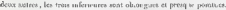

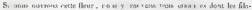

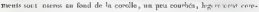

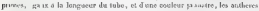

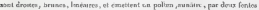
pliodes it leur faos esiono enire Jee éthia

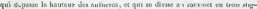

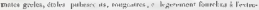

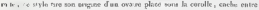

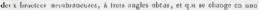

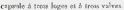

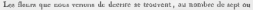

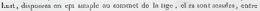

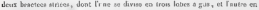

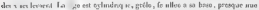

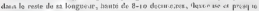

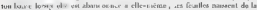

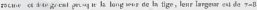

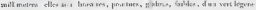

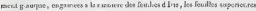

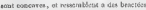




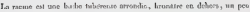

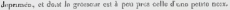

\section{15 T O I A R}

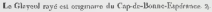

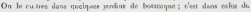

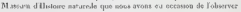

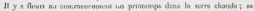

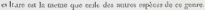

$$
\text { Q BSERPATIOAS }
$$

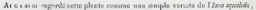

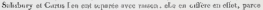

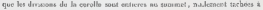

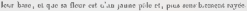

\section{LXPLICAIIOX DE LA PLANCLE}

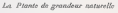

I Lea buteris.

3 La corcuk anvere

4 Le pisist 


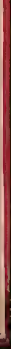




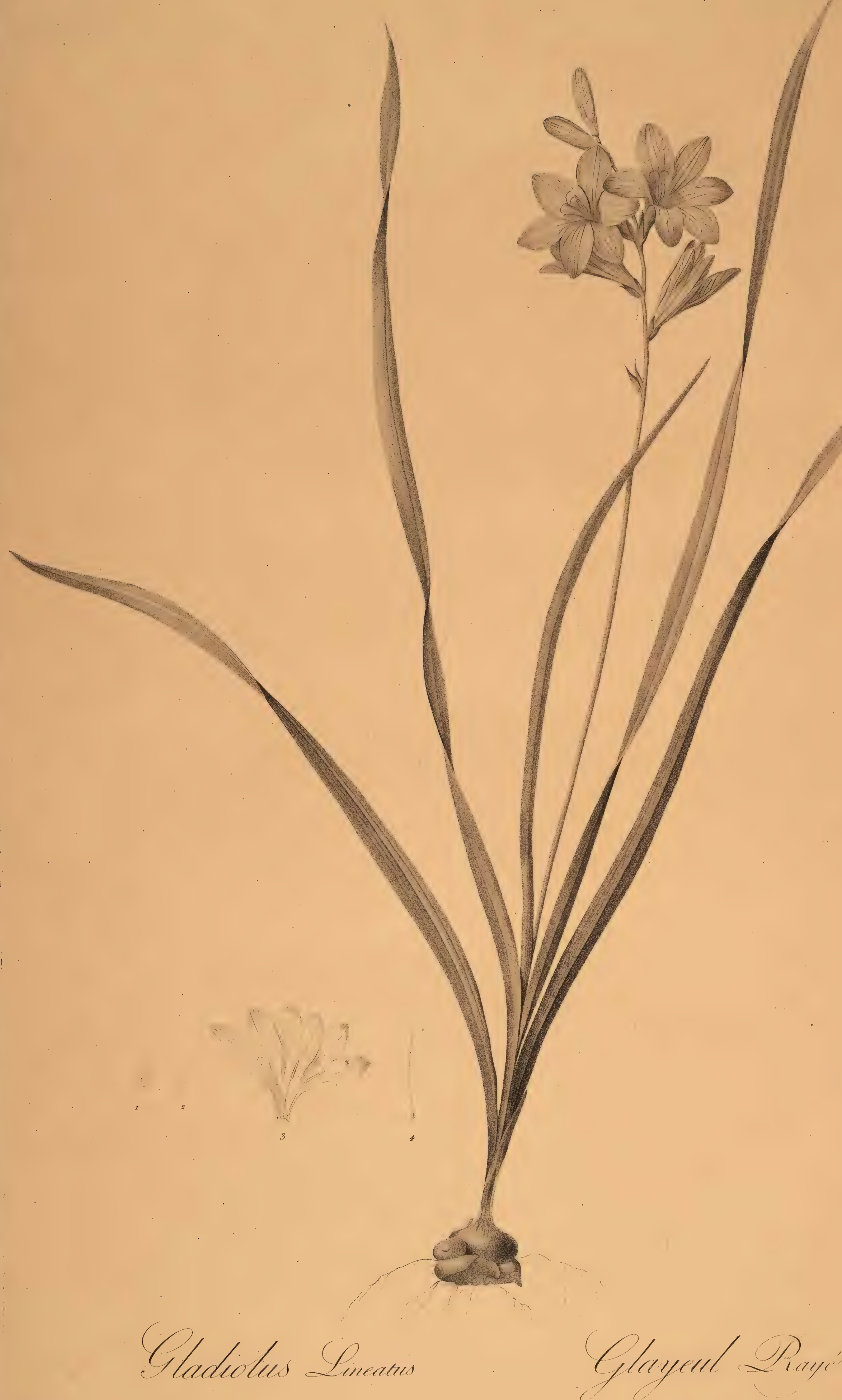





\title{
MOROEA VAGINATA.
}

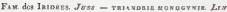

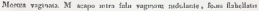

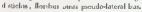

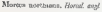

\section{MOREE A LONGLE GAINE}

\section{I. 5 C I I P TIO}

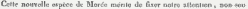

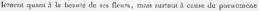

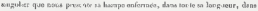

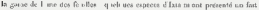

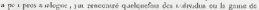

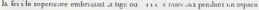

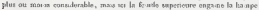

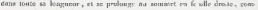

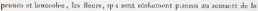

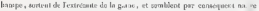

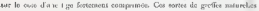

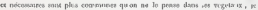

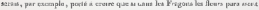

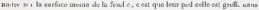

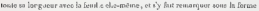

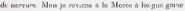

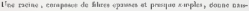

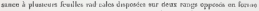

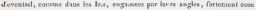

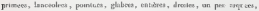

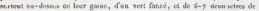
meivace t

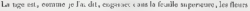

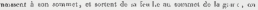

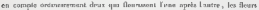

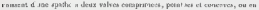

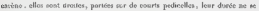

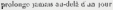




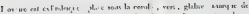

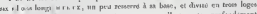

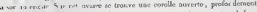

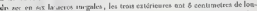

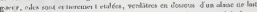

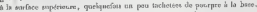

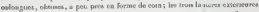

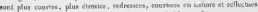

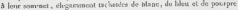

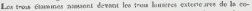

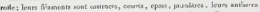

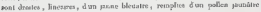
lygurcanrat vavqueus

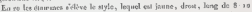

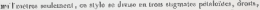

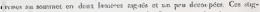

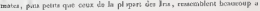
enais des 5 cousers stie

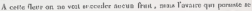

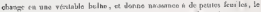

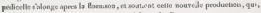

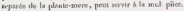

\section{I S T O I E T}

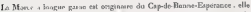

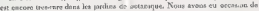

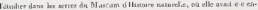

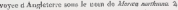

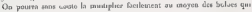
neendeot aus Aletrs.

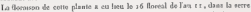

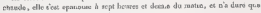

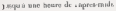

\section{IXILICA T IOX DELA FLANC II}

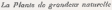

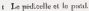




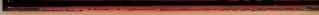




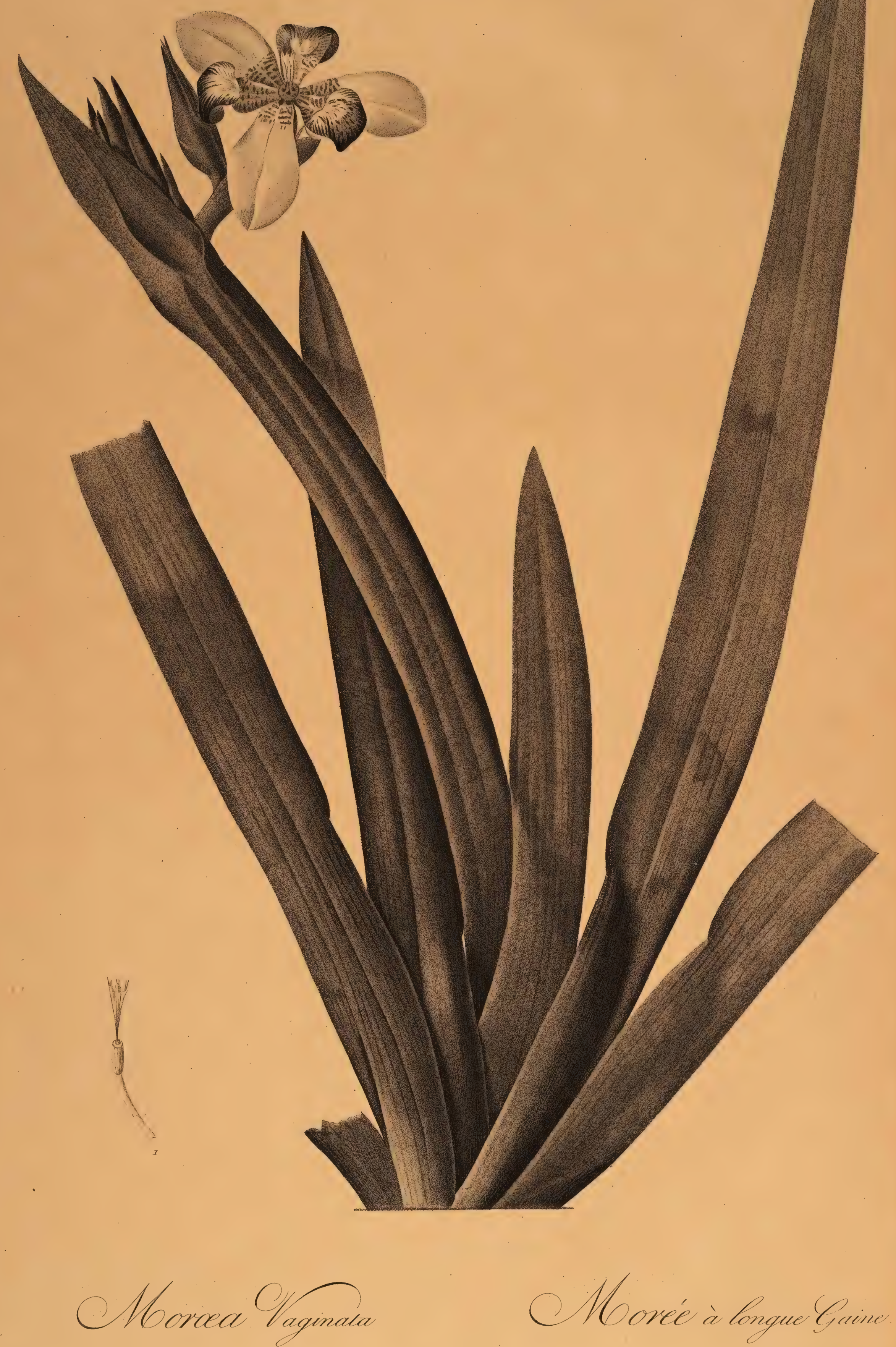




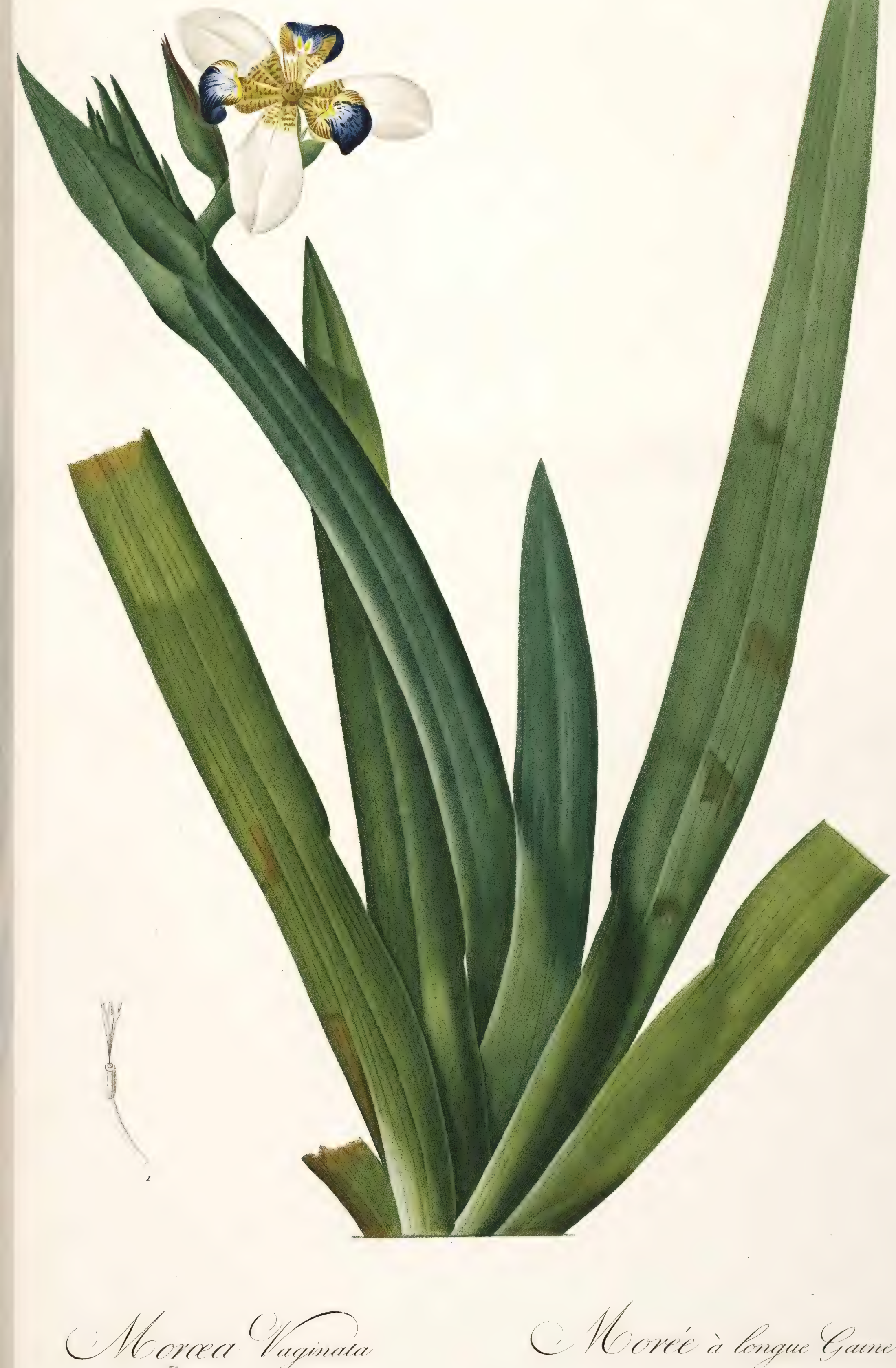




\section{MIAANTA ARTNDTVACEA.}

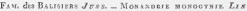

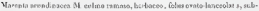

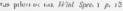

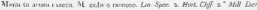

K. I Ginoh and ne dis

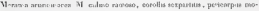

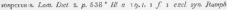

M.

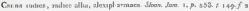

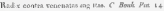

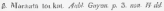

\section{IARAXTA TOSEAL.}

\section{DESORTTT:ON}

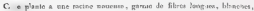

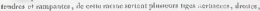

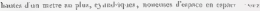

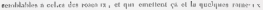

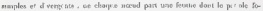

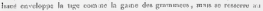

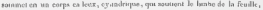

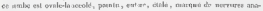

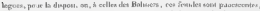

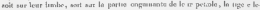

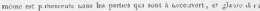

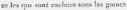

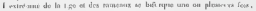

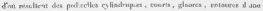

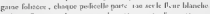

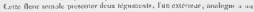

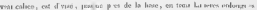

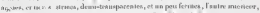

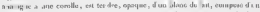

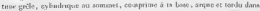

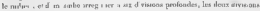

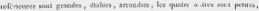
estong nus, bov weoct. 


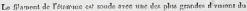

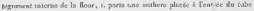

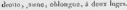

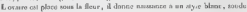

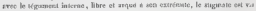

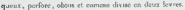

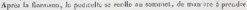

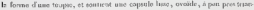

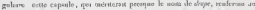

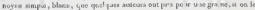

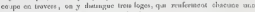
xrusar deus de cen granes arotent $k$ plas eouroat

\section{ก t 5 T 01 if $x$.}

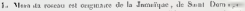

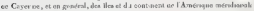

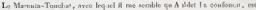

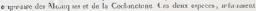

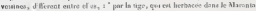

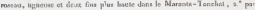

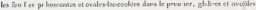

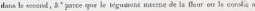

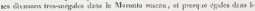

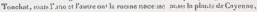

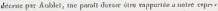

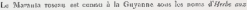

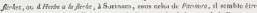

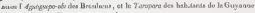

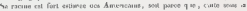

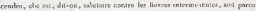

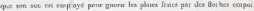

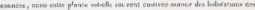

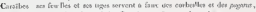

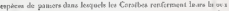

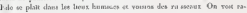

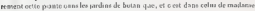
Becapario que num abed e 1 ectwion oo Jolkerver

\section{EXPLICAT1OSDELA PLANCHA.}

1. Les tran scgiments esbernes de Te Mear = Les segoenbs iniernes 


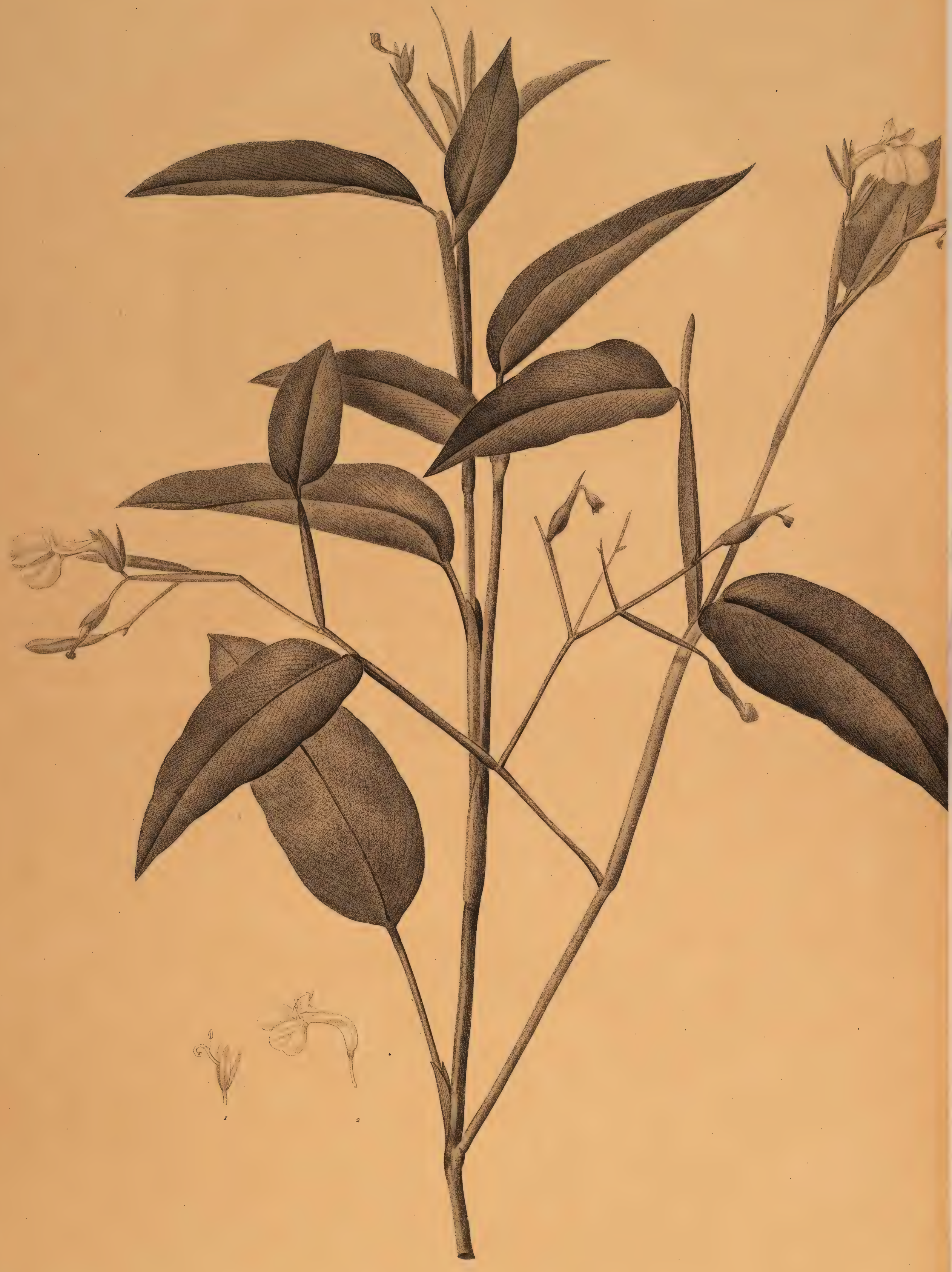

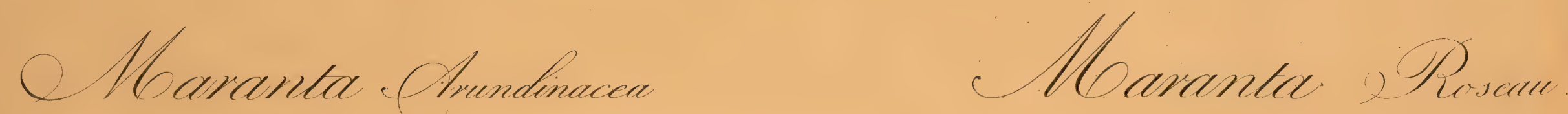




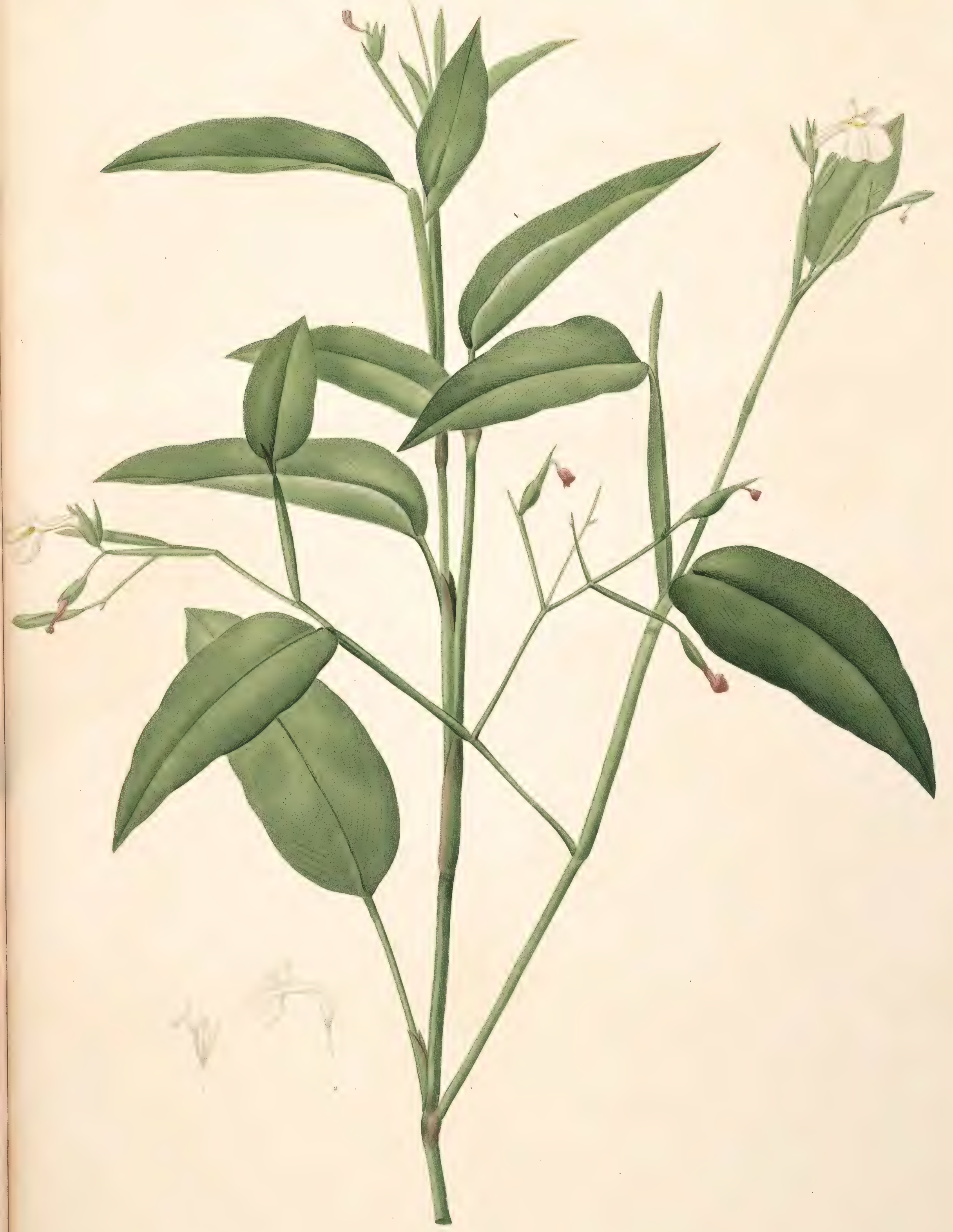

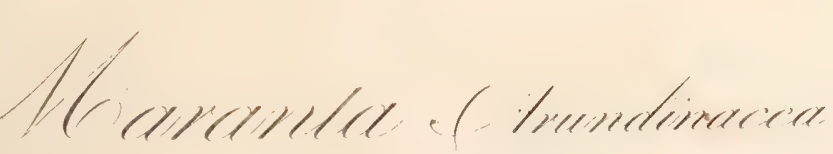

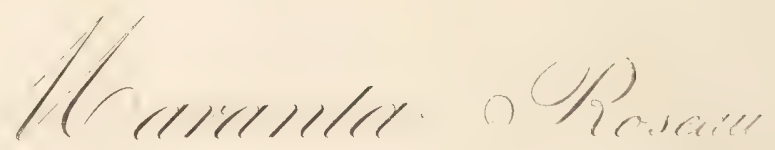





\title{
A NTHERICUY MILIEFLORTM
}

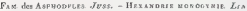

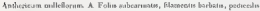

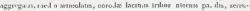
nib, exirrn's, nikexis, Ea 1 gono

\section{ANTHERIC A MILIE IJFERS}

\section{DESE EIPTIOA}

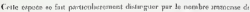

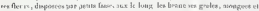

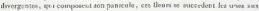

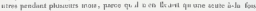
dans divique if 'or pe

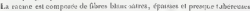

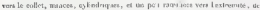

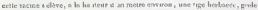

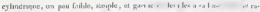

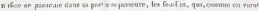

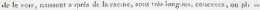

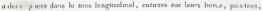

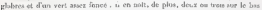

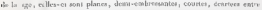
eba, of momb at ex a des braciese

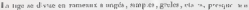

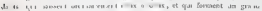

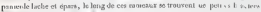

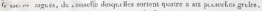

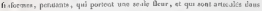
Le aniver coonme racas les Aproges

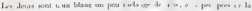

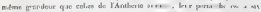

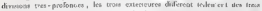

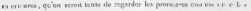

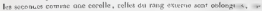

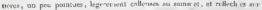

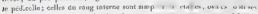

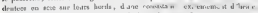

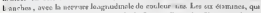

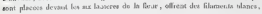




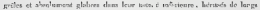

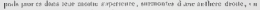

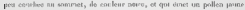

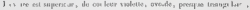

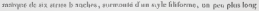

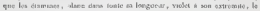

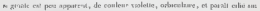

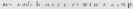

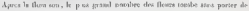

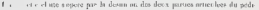

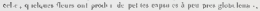

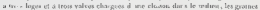

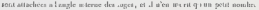
tame chathat capilik.

\section{T 0 I $\mathrm{E}$}

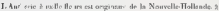

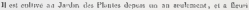
cons le ont cide lae posit

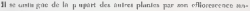

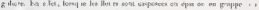

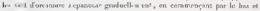

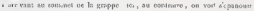

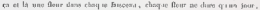

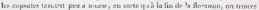

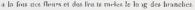

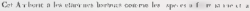

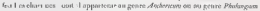

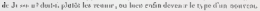

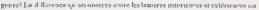

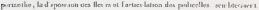

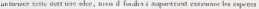

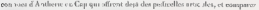

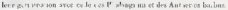

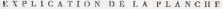

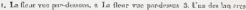

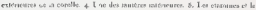

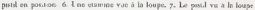
D. La mijurli g Let grinses. 


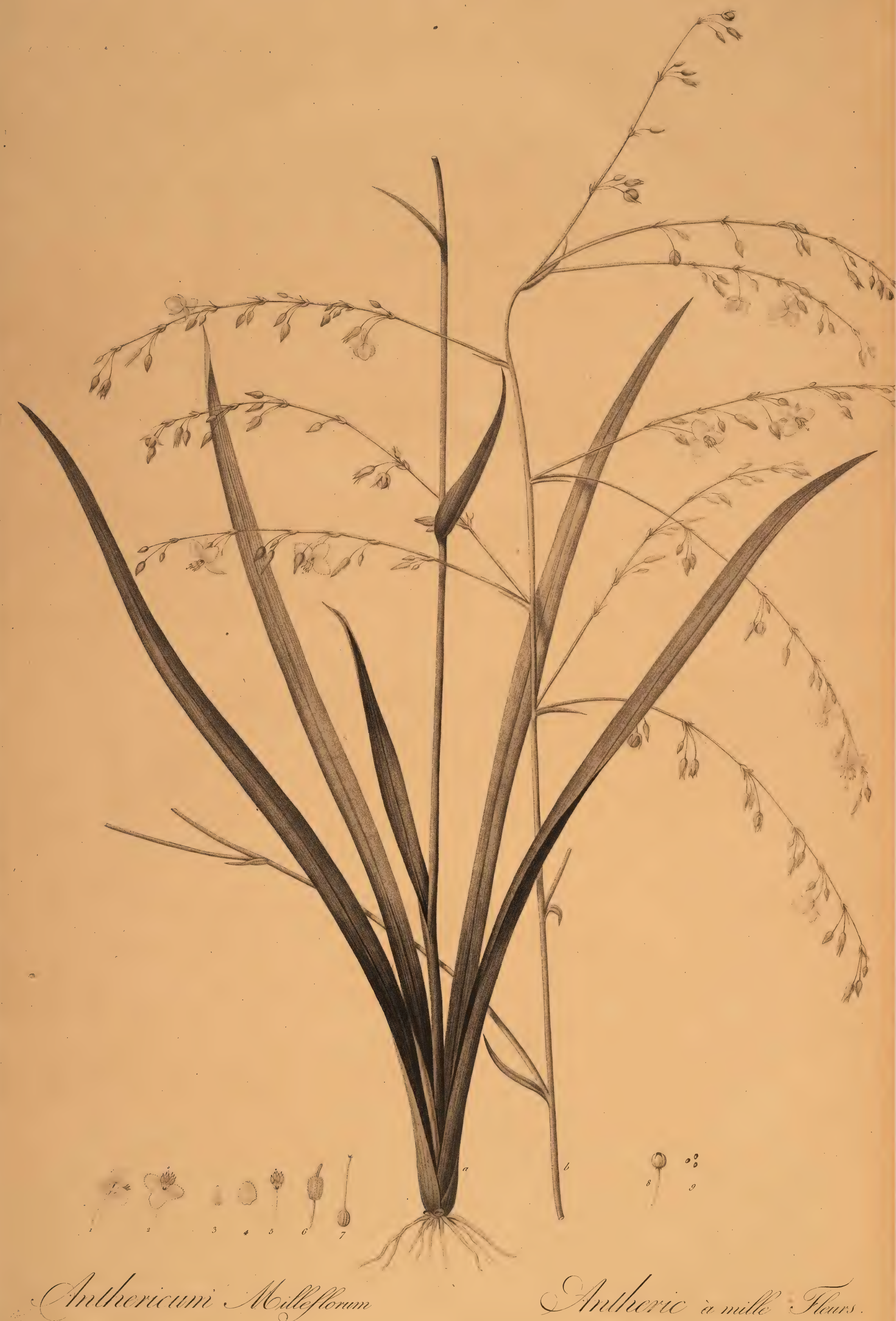





\title{
LACMENALTA LA NCEAFOLIA.
}

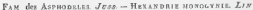

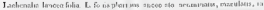

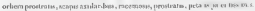

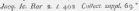

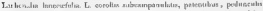

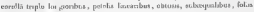

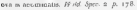

\section{ACUFV ALE EN FORYE DE LANGE}

\section{DESCIIPTIO}

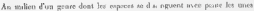

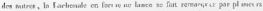

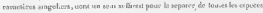
ction, it:

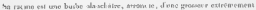

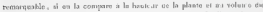

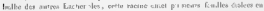

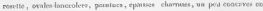

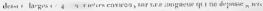

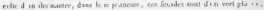

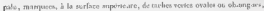

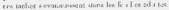

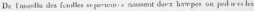

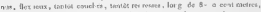

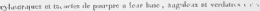

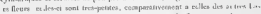

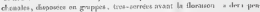

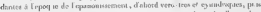

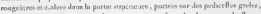

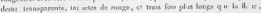

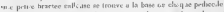

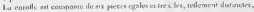

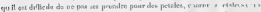

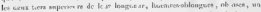

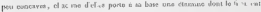




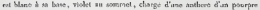

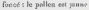

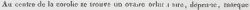

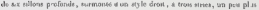

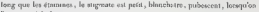

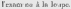

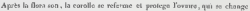

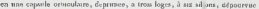

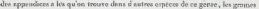

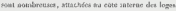

\section{if 1 S 10 I R I}

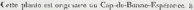

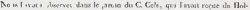

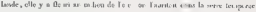

$$
O A \& 8 \pi P+T \& O N \&
$$

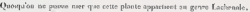

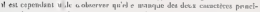

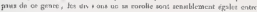

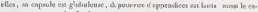

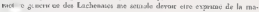
rier smatist

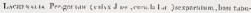

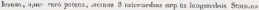

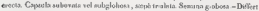

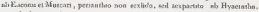

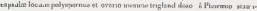

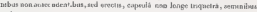
grotiocen

\section{I LICATION BE IA PLA A H E.}

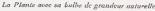

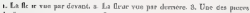

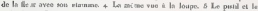

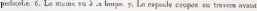
a natural. 


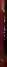




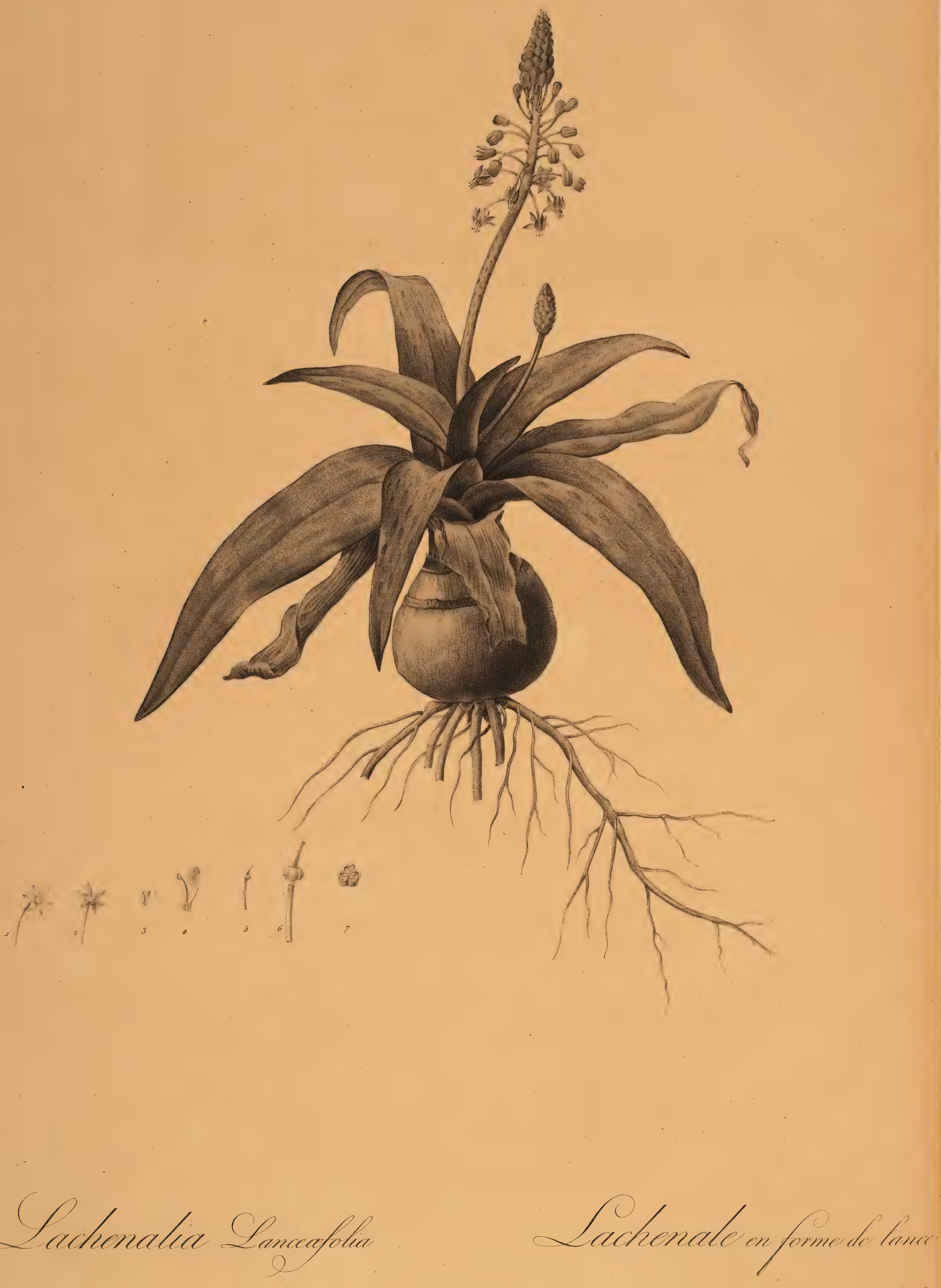




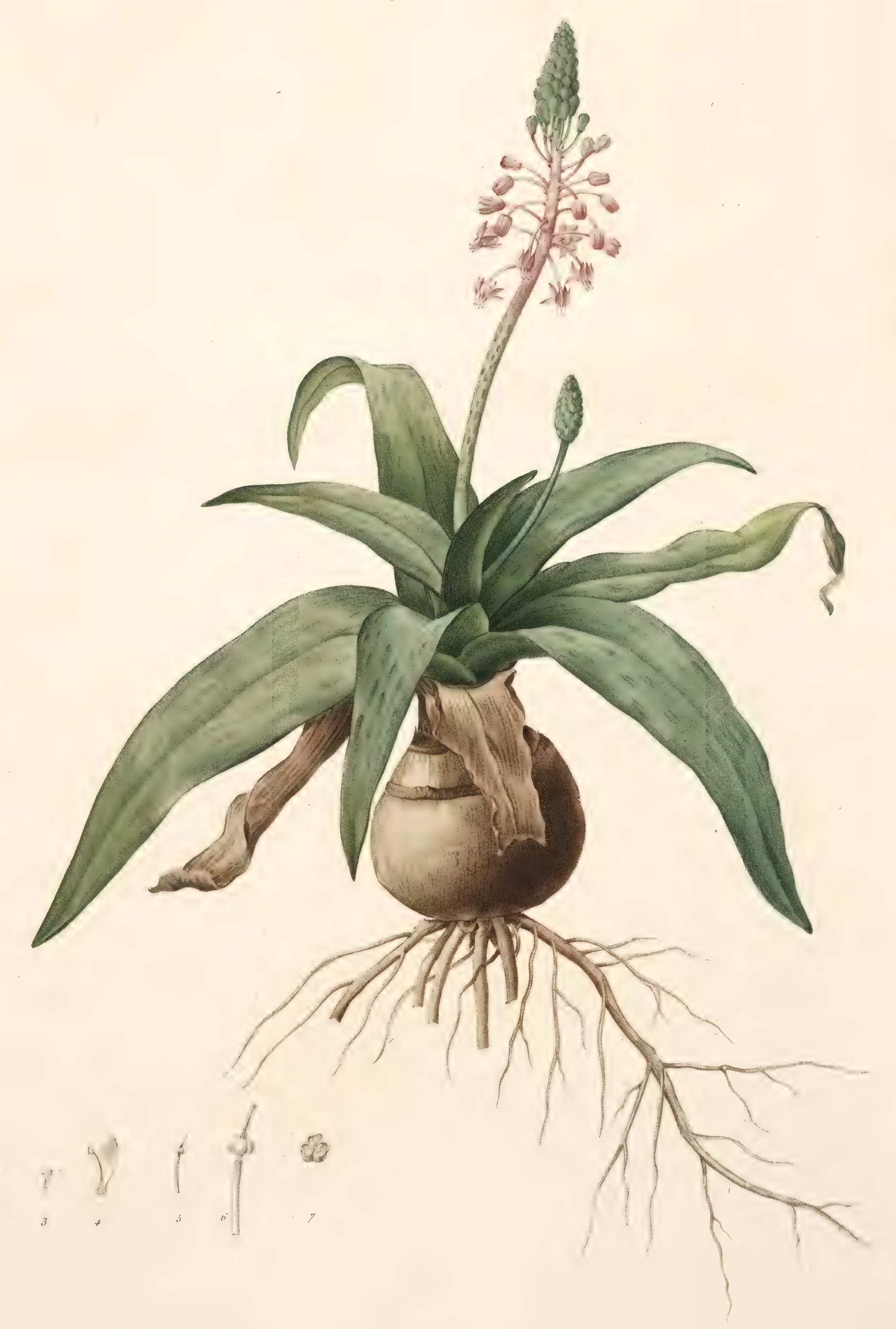

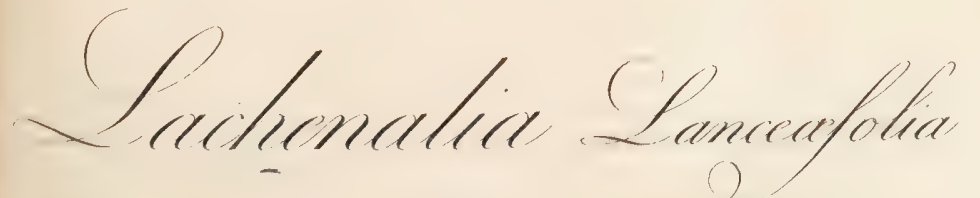

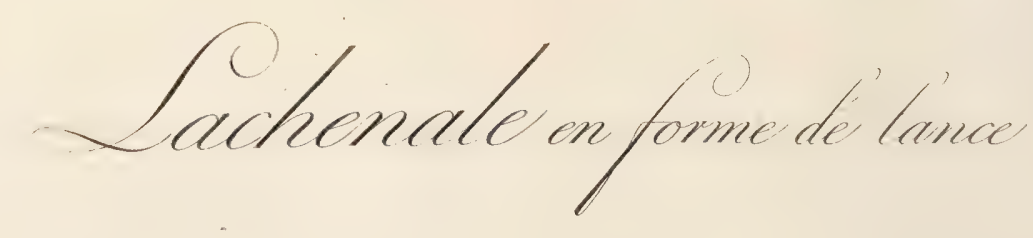





\title{
CLOBBA NUTANS.
}

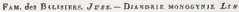

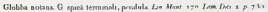

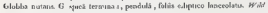
lion 1. P. 1.53

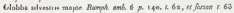

\section{CLOBBEE PBNDANTI.}

\section{E \& G I I P T I O}

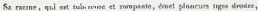

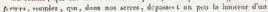

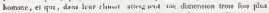

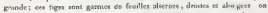

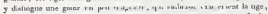

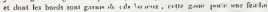

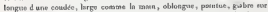

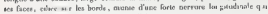

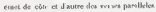

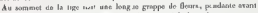

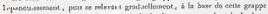

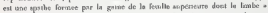

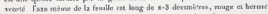

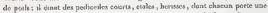

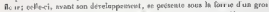

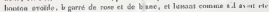

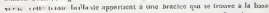

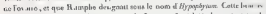

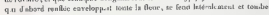
d kle moin..

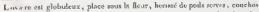

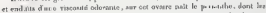

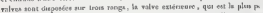

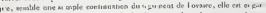

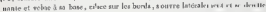

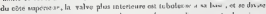

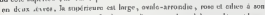

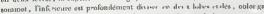

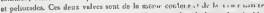

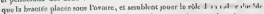

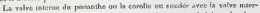

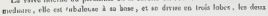




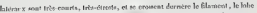

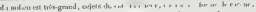

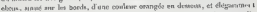

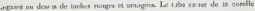

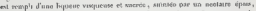

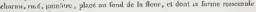
a i leatin ar

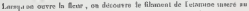

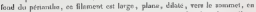

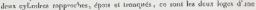

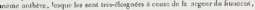
st que unt cLt prase mal-a prepos paer deut ashores dislieirs

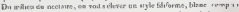

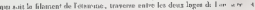

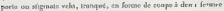

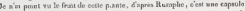

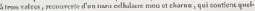

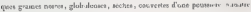

\section{il $T$ \& $T$, 0 I $\mathrm{A}-\mathrm{F}$}

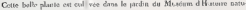

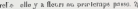

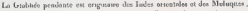

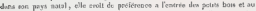

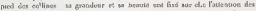

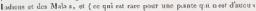

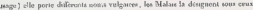

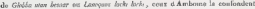

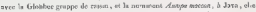

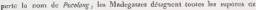

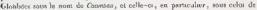

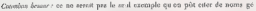

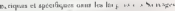

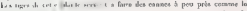

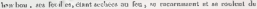

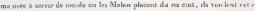

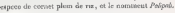

\section{EXFLTC\&TION DE LA PLANCAR.}

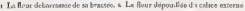

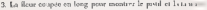




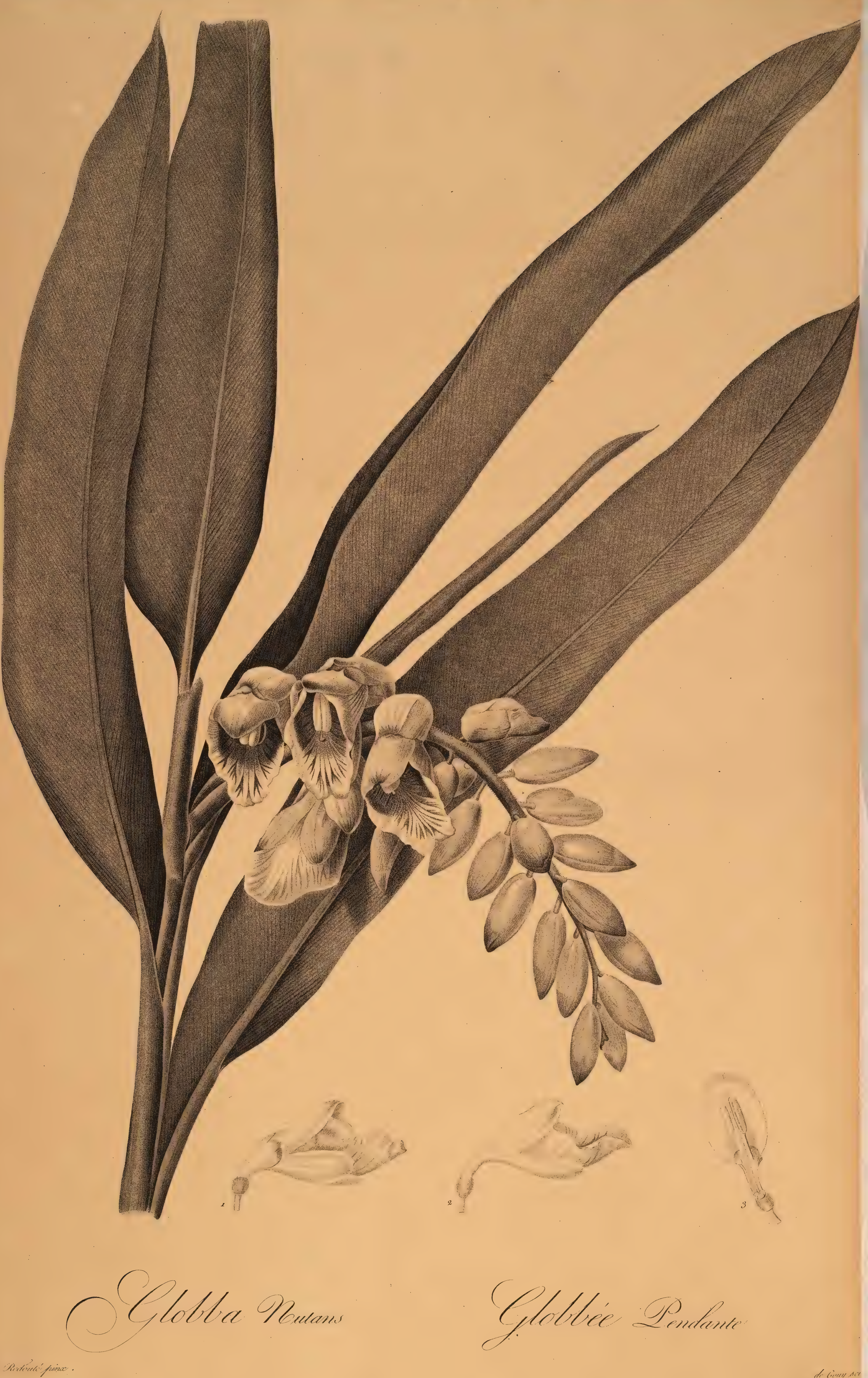




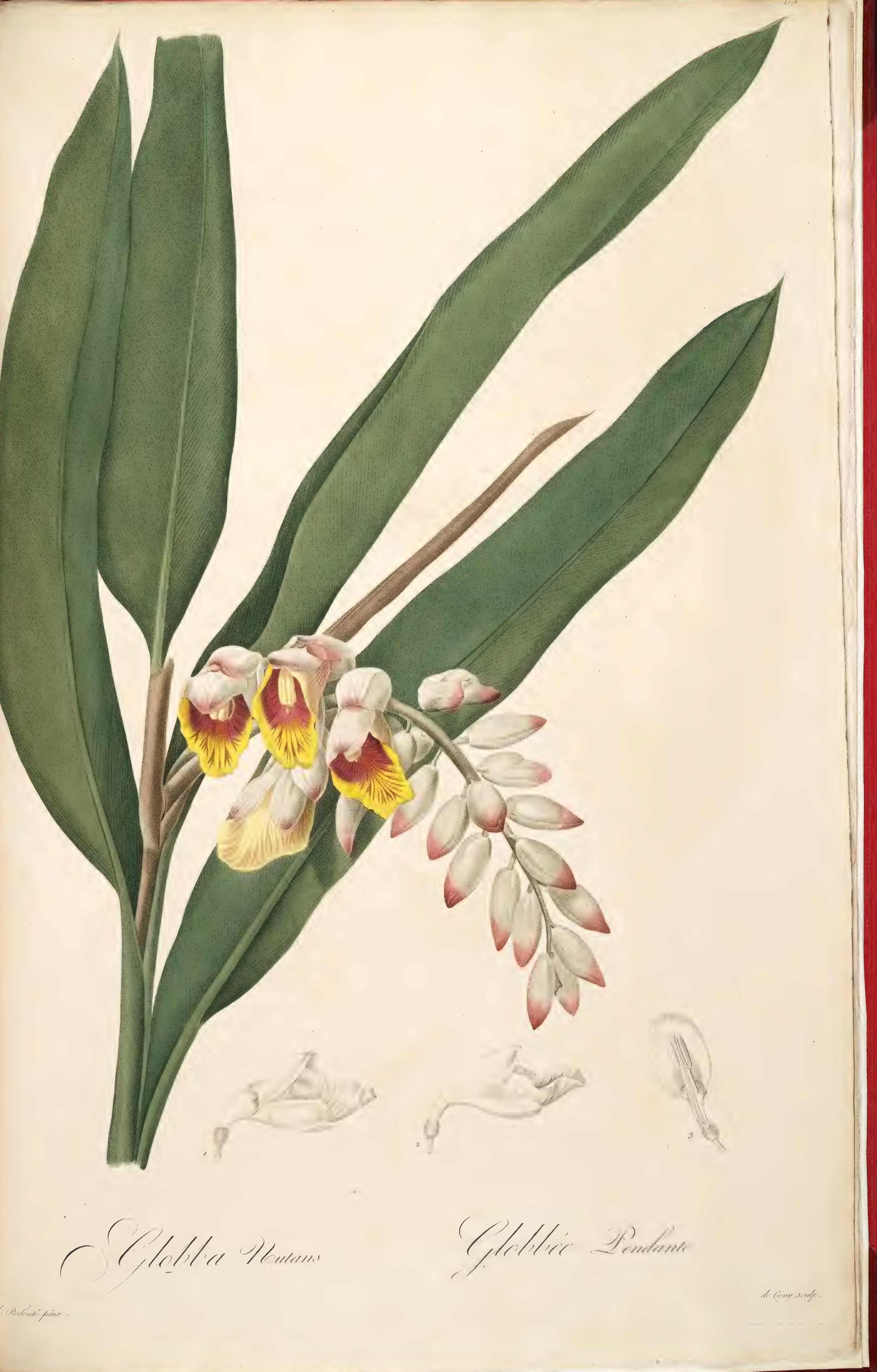




\section{ADDITIONS ET CORRECTIONG.}

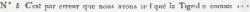

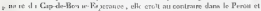

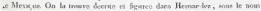

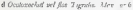

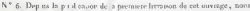

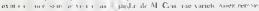

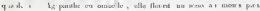

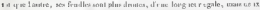

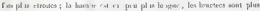

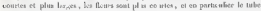

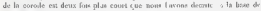

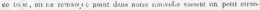

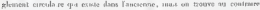

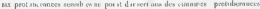

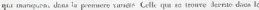

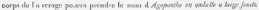

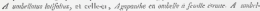

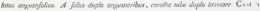

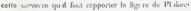

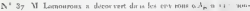

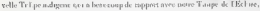

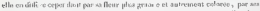

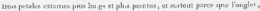

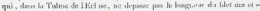

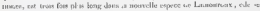

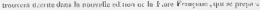

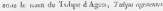

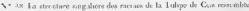

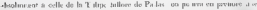

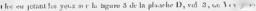

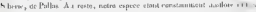
scudcuanent to sul.e de Hat lat 


\section{T A B L B F A A N C A I S E}

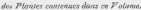

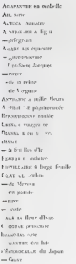

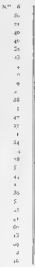
Hinatadekitas
rat r portice
iscinelisubr
$x$ Tho

- Nomier

Ais bifiene

+ tirpue Arr

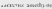

Kevrovar irtert

woatide mi bime of lones

ver

- encrear

reanor

1 means is rase o :

ton proroo

Niwaste Intaue

Nimersalet lisiax oh.

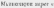

vow bur ir

porimat?

Mide. b bescer nriu

- Gaverin

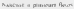

$$
\text { atherven }
$$

tevisurs ase tis

suloo in thos

- iltarirus

ixame trint in pom

Tos in se I Inated

ate Cro

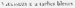




\section{TA BLE LATINE}

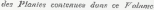

Aeast a ristion

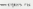

rowitereis

- ivprer

- Cocumia

4 พา

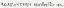

A.s.t. mine

buth 4 stiritate

by Tirmen riblisan

A nierspe rowari

heviva ase a ligs

-rirgia

r res poup a nerabos

mill rave

Crsix veldesto

Bagoin m-the

nimes.

- nvosaminter

-

Frepras nares.

For anis tis

vilase sation

cisia.

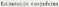
Ieli an!

- Inmatra-

- torwios

ch:

Giantar niforr.

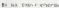

d inys al ines.

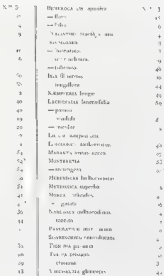




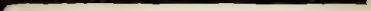







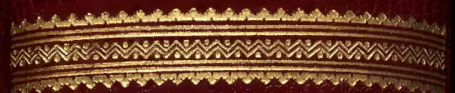

P. J. REDOUTÉ sक

LES

LILIACÉES
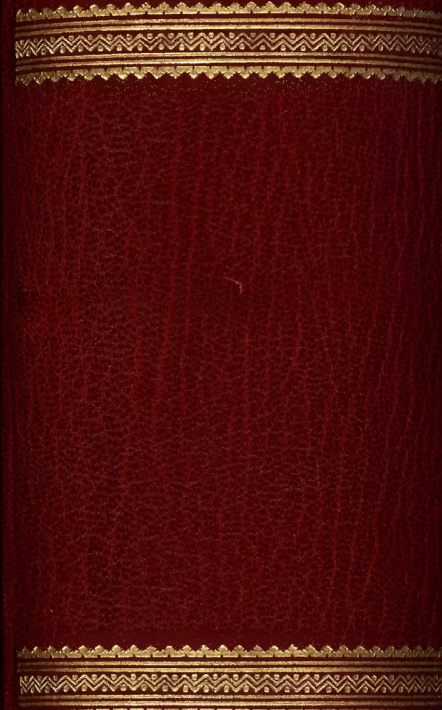

1
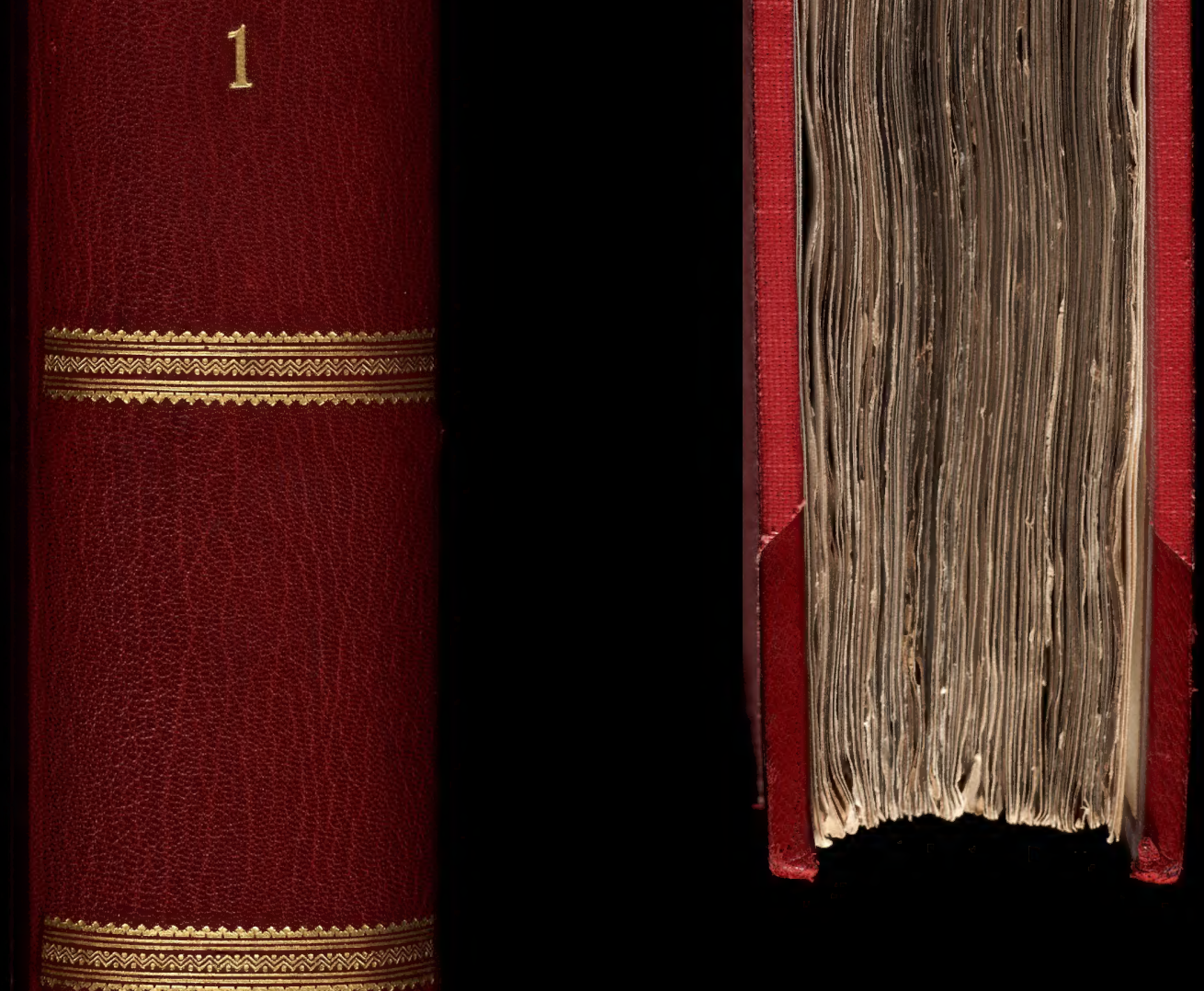

Top

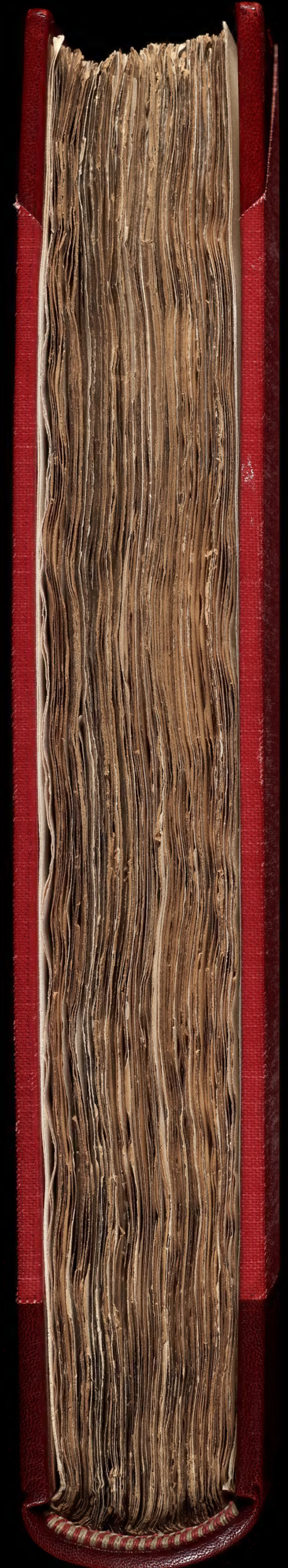

Foot

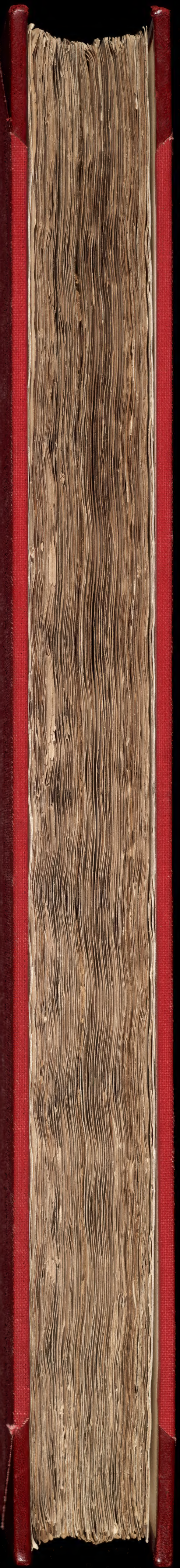



